LTH 857

arXiv:0912.0369 [hep-ph]

DESY 09-211

SFB/CPP-09-119

NIKHEF 09-031

\title{
On Higgs-exchange DIS, physical evolution kernels and fourth-order splitting functions at large $x$
}

\author{
G. Soar ${ }^{a}$, S. $\operatorname{Moch}^{b}$, J.A.M. Vermaseren ${ }^{c}$ and A. $\operatorname{Vogt}^{a}$ \\ ${ }^{a}$ Department of Mathematical Sciences, University of Liverpool \\ Liverpool L69 3BX, United Kingdom \\ ${ }^{b}$ Deutsches Elektronensynchrotron DESY \\ Platanenallee 6, D-15735 Zeuthen, Germany \\ ${ }^{c}$ NIKHEF Theory Group \\ Science Park 105, 1098 XG Amsterdam, The Netherlands
}

\begin{abstract}
We present the coefficient functions for deep-inelastic scattering (DIS) via the exchange of a scalar $\phi$ directly coupling only to gluons, such as the Higgs boson in the limit of a very heavy top quark and $n_{f}$ effectively massless light flavours, to the third order in perturbative QCD. The two-loop results are employed to construct the next-to-next-to-leading order physical evolution kernels for the system $\left(F_{2}, F_{\phi}\right)$ of flavour-singlet structure functions. The practical relevance of these kernels as an alternative to $\overline{\mathrm{MS}}$ factorization is bedevilled by artificial double logarithms at small values of the scaling variable $x$, where the large top-mass limit ceases to be appropriate. However, they show an only single-logarithmic enhancement at large $x$. Conjecturing that this feature persists to the next order also in the present singlet case, the three-loop coefficient functions facilitate exact predictions (backed up by their particular colour structure) of the double-logarithmic contributions to the fourth-order singlet splitting functions, i.e., of the terms $(1-x)^{a} \ln ^{k}(1-x)$ with $k=4,5,6$ and $k=3,4,5$, respectively, for the off-diagonal and diagonal quantities to all powers $a$ in $(1-x)$.
\end{abstract}




\section{Introduction}

Structure functions in lepton-nucleon deep-inelastic scattering are classic observables probing Quantum Chromodynamics (QCD), the theory of the strong interaction, and in particular the structure of the nucleon. Indeed, structure function measurements provide the backbone of our knowledge of the quark and gluon longitudinal momentum distributions in the proton to this day, 40 years after the pioneering measurements of DIS at SLAC [1], see also Refs. [2]. In turn these distributions, in short referred to as the parton distributions or parton densities of the nucleon, are indispensable for the description and analysis of hard scattering processes at the proton colliders forming the high-energy frontier of particle physics, i.e., the TEVATRON and the LHC. See, e.g., Ref. [3] for introductory overviews and the present status of structure functions and QCD.

The asymptotic freedom of QCD [4] facilitates a partly perturbative description of hard hadron processes. At all practically relevant scales, however, the strong coupling constant $\alpha_{\mathrm{S}}$ is much larger than its electroweak counterparts. Hence calculations to a higher order are required in perturbative QCD in order in arrive at precise predictions. Also in this respect structure functions form a special tool, since they can be expressed in terms of the operator-product expansion [5] which provides a framework that renders higher-order calculations considerably more accessible.

In a series of previous articles, three of us have exploited this fact to derive the exact thirdorder QCD corrections to the splitting functions governing the scale dependence of the parton distributions $[6,7]$ - and thus the transfer of information from DIS at moderate scales to the high scales involved in Higgs, gauge boson, top quark and new physics processes at TEVATRON and the LHC - and to the partonic cross sections (coefficient functions) for the most important structure functions [8-10]. As all modern higher-order calculations in perturbative QCD, those calculations have been performed in dimensional regularization [11], and the splitting functions and coefficient functions have been determined in the modified [12] minimal subtraction [13] scheme, $\overline{\mathrm{MS}}$.

Consequently the higher-order parton densities are not defined in terms of any physical process, but by a mathematical description employed for isolating the mass singularities which lead to their scale dependence. The $\overline{\mathrm{MS}}$ scheme has definite advantages, e.g., it leads to a stable (orderindependent) functional form of the dominant diagonal (quark-quark and gluon-gluon) splitting functions in the limit of large momentum fractions $x$ [14], see also Ref. [15], a feature that assists a stable evolution of the parton densities over the wide range of scales mentioned above.

An alternative approach has been suggested already long ago in Ref. [16], see also Refs. [17]: As discussed below, the parton densities can be eliminated from the description of the structure functions (and other hard processes). The dependence of the observables on the physical hard scale (in DIS $Q^{2}=-q^{2}$, where $q$ is the four-momentum of the exchanged electroweak gauge boson) is then given in terms of so-called physical evolution kernels or physical anomalous dimensions. While the direct relation between different observables via the universal parton densities is obscured in this approach, it can be practically useful for analyses of selected observables such as the determination of $\alpha_{\mathrm{s}}$ from the scaling violations of structure functions [18]. 
The construction of physical evolution kernels is particularly simple (and unique) for flavour non-singlet, i.e., gluon insensitive observables. It turns out that these kernels show an only singlelogarithmic enhancement at large values of the scaling variable $x$ for a wide range of non-singlet quantities in DIS, semi-inclusive $e^{+} e^{-}$annihilation (SIA) and the Drell-Yan process [19,20]. This general behaviour is in contrast to that of all corresponding $\overline{\mathrm{MS}}$ coefficient functions which receive double-logarithmic contributions. As a result the physical kernels provide an - at present not yet formally proven - all-order exponentiation of the highest $\ln (1-x)$ terms beyond the leading $(1-x)^{-1}$ contributions covered by the standard threshold resummation [21] to all powers in $(1-x)$.

In the present article we address the flavour singlet case which requires two observables and a $2 \times 2$ matrix evolution kernel. Unlike Refs. $[17,18]$ we study the ideal - for theoretical purposes gluonic complement to the most important gauge-boson exchange structure function $F_{2}$, i.e., DIS by the exchange of a scalar $\phi$ coupling directly only to gluons via an additional term $\phi G^{\mu v} G_{\mu \nu}$ in the Lagrangian, where $G^{\mu v}$ represents the gluon field strength tensor. Such a term, already suggested as a trick in Ref. [16], is of course not present in the fundamental Standard Model Lagrangian. However, it does occur effectively for the Higgs boson in the limit of a very heavy top quark [22].

This interaction was included, in order to directly access also the quark-gluon and gluon-gluon splitting functions, in the calculations of Ref. [7] and, in fact, its precursors [23,24]. Hence the oneand two-loop coefficients functions for the resulting structure function $F_{\phi}$ were calculated, but not published, quite a while ago. The more recent, but also unpublished three-loop results have already been employed to determine the third-order gluon jet function in threshold resummation [25] and the pole terms of the three-loop gluon form factor [26] - and from the latter the next-to-nextto-next-to-leading logarithmic $\left(\mathrm{N}^{3} \mathrm{LL}\right)$ threshold resummation for Higgs production in the heavytop limit [27]. These coefficient functions also provide, via an analytic continuation to timelike kinematics, a strong check of the next-to-next-to-next-to-leading order $\left(\mathrm{N}^{3} \mathrm{LO}\right)$ computation in Ref. [28] of the top-induced Higgs decay into hadrons [29]. The first- and second-order coefficient functions for $F_{\phi}$ have now been derived independently in a completely different manner [30].

The outline of this article is as follows. In Section 2 we introduce the formalism used for calculating the coefficient functions for $F_{\phi}$ to order $\alpha_{\mathrm{s}}^{3}$, briefly discuss the diagram calculation and its checks, and provide the transformation to the physical kernels to the fourth order. In Section 3 we then present the exact expressions for the coefficient functions and address their size and endpoint behaviour. The one- and two-loop results are then combined in Section 4 with the known second- and third-order splitting functions to derive the next-to-leading order (NLO) and next-tonext-to-leading order (NNLO) physical kernels for the system $\left(F_{2}, F_{\phi}\right)$. Also these kernels show an only single-logarithmic large- $x$ enhancement to all powers in $(1-x)$, a feature that we conjecture to hold also at the next order. In Section 5 this conjecture and the three-loop coefficient functions of Section 3 are then employed to predict the coefficients of the highest three powers of $\ln (1-x)$ in all four singlet splitting functions at order $\alpha_{\mathrm{s}}^{4}\left(\mathrm{~N}^{3} \mathrm{LO}\right)$. We summarize our results and give a brief outlook in Section 6. Appendices A and B contain the partly very long expressions, respectively, for the new third-order coefficient functions and the elements of the NNLO physical kernel matrix. In Appendix $\mathrm{C}$ we finally provide the large- $x$ limit of the gluon coefficient functions for $F_{2}$. 


\section{Formalism and calculation}

We consider inclusive deep-inelastic lepton-nucleon scattering through the exchange of a scalar (Higgs) boson $\phi$, which proceeds through the reaction

$$
\phi(q)+\operatorname{nucl}(p) \rightarrow X
$$

Here $X$ denotes all hadronic final states allowed by quantum number conservation. The boson $\phi$ transfers a space-like momentum $q$ (i.e., $Q^{2} \equiv-q^{2}>0$ ), while the nucleon carries momentum $p$. The scaling variable of the reaction is defined as usual as $x=Q^{2} /(2 p \cdot q)$ with $0<x \leq 1$.

In complete analogy to ordinary (gauge-boson exchange) DIS, the cross section for this process can be parametrized in terms of a structure function $F_{\phi}$. Through the optical theorem the total cross section (hence the structure function $F_{\phi}$ ) is related to the imaginary part of the forward amplitude $\mathcal{T}_{\phi}$ for the scattering process in Eq. (2.1) of a virtual Higgs boson off the nucleon,

$$
\mathcal{T}_{\phi}(p, q)=i \int d^{4} z e^{i q z}\langle\text { nucl, } p|T(J(z) J(0))| \text { nucl, } p\rangle .
$$

This quantity contains the time-ordered product of two scalar currents (see below) to which the standard operator-product expansion (OPE) can be applied in the Bjorken limit (large $Q^{2}$ for fixed $x$ ). The relevant steps have been already discussed in previous lower-order and fixed Mellin- $N$ third-order calculations, see Refs. [23,24], hence we can be brief in recalling some key issues.

The OPE decomposes the current product in Eq. (2.2) in terms of the standard set of spinaveraged matrix elements of the (renormalized) spin- $N$ twist-two irreducible flavour-singlet quark and gluon operators

$$
\left\langle\text { nucl, } p\left|O_{i}^{\left\{\mu_{1}, \ldots, \mu_{N}\right\}}\right| \text { nucl, } p\right\rangle=p^{\left\{\mu_{1}\right.} \ldots p^{\left.\mu_{N}\right\}} A_{i, \text { nucl }}\left(N, \mu^{2}\right)
$$

and the respective hard scattering parton coefficient functions $C_{\phi, i}$ with $i=\mathrm{q}$, g, where $\mu$ stands for the renormalization scale. The operators $O_{\mathrm{q}}$ and $O_{\mathrm{g}}$ in Eq. (2.3) arise from the symmetric and traceless part of $N$ covariant derivatives $D^{\mu}$, respectively acting on the quark $(\psi)$ and gluon fields $\left(G^{\mu v}\right)$. Neglecting $1 / Q^{2}$ power corrections, the OPE applied to Eq. (2.2) allows us to express the Mellin- $N$ moments of the structure function $F_{\phi}$ in terms the matrix elements $A_{i, \text { nucl }}$ and coefficient functions $C_{\phi, i}$ as

$$
\begin{aligned}
F_{\phi}\left(N, Q^{2}\right) & =\int_{0}^{1} d x x^{N-1} F_{\phi}\left(x, Q^{2}\right) \\
& =\sum_{i=\mathrm{q}, \mathrm{g}} C_{\phi, i}\left(N, \frac{Q^{2}}{\mu^{2}}, \alpha_{\mathrm{s}}\right) A_{i, \mathrm{nucl}}\left(N, \mu^{2}\right) .
\end{aligned}
$$

Here the first line (2.4) fixes our conventions for Mellin moments $N$ and the second relation (2.5) holds (due to symmetry properties for $x \rightarrow-x$ ) for even-integer values of $N$. All (complex) moments, and the complete $x$-dependence, are uniquely fixed by analytic continuation though. 
The renormalization of the singlet operators $O_{\mathrm{q}}$ and $O_{\mathrm{g}}$ in Eq. (2.3) in terms of their bare counterparts proceeds as

$$
O_{i}=Z_{i k} O_{k}^{\text {bare }},
$$

where the renormalization factors $Z_{i k}$ are matrix-valued (i.e., summation of $k$ is understood) and the dependence on $N$ has been suppressed here (and in the following) for brevity. The well-known anomalous dimensions $\gamma_{i k}$ arise from the renormalization factors $Z_{i k}$ as

$$
\gamma_{i k}=-\left(\frac{d}{d \ln \mu^{2}} Z_{i j}\right)\left(Z^{-1}\right)_{j k}
$$

and the scale dependence of the operators $O_{i}$ is given by

$$
\frac{d}{d \ln \mu^{2}} O_{i}=-\gamma_{i k} O_{k} \equiv P_{i k} O_{k}
$$

Eq. (2.8) also recalls the conventional relation between the anomalous dimensions and the moments of the splitting functions $P_{i k}(x)$.

Using dimensional regularization [11] in $D=4-2 \varepsilon$ dimensions and the modified minimal subtraction scheme $\overline{\mathrm{MS}}[12,13]$, the renormalization factors $Z_{i k}$ in Eq. (2.6) are a given by a series of poles in $1 / \varepsilon$ which can be expressed in terms of the perturbative expansion coefficients $\gamma^{(l)}$ of the anomalous dimensions and the coefficients $\beta_{\mathrm{n}}$ governing the running coupling. In a power expansion in $\alpha_{\mathrm{s}}$ the former can be written as

$$
\gamma_{i k}=\sum_{l=0}^{\infty} a_{\mathrm{s}}^{l+1} \gamma_{i k}^{(l)}
$$

while the latter are given by

$$
\frac{d}{d \ln \mu^{2}} \frac{\alpha_{\mathrm{s}}}{4 \pi} \equiv \frac{d a_{\mathrm{s}}}{d \ln \mu^{2}}=-\varepsilon a_{\mathrm{s}}-\beta_{0} a_{\mathrm{s}}^{2}-\beta_{1} a_{\mathrm{s}}^{3}-\beta_{2} a_{\mathrm{s}}^{4}-\ldots
$$

Here $\beta_{1, \ldots, 4}$ denote the known four-dimensional expansion coefficients of the beta function of QCD [4, 31-33], $\beta_{0}=11 / 3 C_{A}-2 / 3 n_{f}$ etc, with $n_{f}$ representing the number of active quark flavours and $C_{A}=3$ (and $C_{F}=4 / 3$ ) denoting the usual $\mathrm{SU}\left(n_{c}=3\right)$ colour factors.

The $\mathrm{N}^{l} \mathrm{LO}$ anomalous dimensions $\gamma^{(l)}$ are related to the $1 / \varepsilon$ single poles of $Z_{i k}$ in Eq. (2.6) at order $a_{\mathrm{s}}^{l+1}$. The $D$-dimensional coefficient functions $\widetilde{C}_{\phi, i}$, on the other hand, have an expansion in non-negative powers of $\varepsilon$, viz

$$
\widetilde{C}_{\phi, i}=\delta_{i \mathrm{~g}}+\sum_{l=1}^{\infty} a_{\mathrm{s}}^{l}\left(c_{\phi, i}^{(l)}+\varepsilon a_{\phi, i}^{(l)}+\varepsilon^{2} b_{\phi, i}^{(l)}+\ldots\right)
$$

where $i=\mathrm{q}, \mathrm{g}$ as before, and we have again suppressed the dependence on $N$ (and $Q^{2} / \mu^{2}$ ). Recall that contributions of order $\varepsilon^{k>0}$ in Eq. (2.11) enter the extraction of the anomalous dimensions and coefficient functions at higher orders in $\alpha_{\mathrm{s}}$. For example, as shown in Eq. (2.19) below, the determination of the third-order coefficient functions $c_{\phi, i}^{(3)}$ requires the two-loop $\varepsilon^{1}$ quantities $a_{\phi, i}^{(2)}$. 
For the perturbative determination of splitting functions and coefficient functions the nucleons in Eqs. (2.2) and (2.3) can be replaced by partons. Thus we need to compute all Feynman diagrams for the forward scattering amplitudes which contribute at leading-twist accuracy to the singlet structure functions $F_{\phi}$ in partonic DIS,

$$
T_{\phi, k}(p, q)=i \int d^{4} z e^{i q z}\langle k, p|T(J(z) J(0))| k, p\rangle, \quad k=\mathrm{q}, \mathrm{g},
$$

i.e., we are considering the reactions,

$$
\operatorname{parton}(p)+\operatorname{scalar}(q) \rightarrow \operatorname{parton}(p)+\operatorname{scalar}(q)
$$

where the scalar couples directly only to gluons via a $\phi G^{\mu v} G_{\mu v}$ contribution to the Lagrangian. Such an interaction is effectively included in the Standard Model for the Higgs boson, in the limit of a heavy top quark and negligible Yukawa couplings to all other quark flavours [22]. Actually, the resulting coefficient functions for the Higgs boson differ from those of a scalar with a generic $\phi G^{\mu v} G_{\mu \nu}$ coupling by a perturbative prefactor, known to $\mathrm{N}^{3} \mathrm{LO}$ [34], which is however irrelevant for our considerations. We will return to the limitations of the heavy-top limit in Sections 3 and 4.

The above scalar current in Eq. (2.12) requires an additional, well-known renormalization [35],

$$
\left(G^{\mu v} G_{\mu v}\right)=Z_{G^{2}}\left(G^{\mu v} G_{\mu v}\right)^{\text {bare }}+\ldots, \quad Z_{G^{2}}=\frac{1}{1-\beta\left(a_{\mathrm{s}}\right) /\left(\varepsilon a_{\mathrm{s}}\right)},
$$

which is calculable in terms of the coefficients $\beta_{\mathrm{n}}$ of the QCD beta function (2.10). Moreover the field strength in Eq. (2.14) is subject to operator mixings which, however, give either vanishing contributions to the on-shell matrix elements considered in Eq. (2.13) or, as in case of a quark mass term $m_{\mathrm{q}} \bar{\psi} \psi$, vanish in the present limit of massless quarks.

In order to determine the coefficient functions $C_{\phi, i}$ to order $\alpha_{\mathrm{s}}^{3}$, we thus 'only' need to evaluate the forward scattering amplitudes (2.13) to three loops. The corresponding Feynman diagrams have been generated automatically with QGRAF [36]. The resulting number of diagrams is shown in Table 1. Note that, as already in Refs. [23], the generation and counting of the diagrams are non-standard, as some tricks (e.g., using symmetries valid only at the relevant even values of $N$ ) have been employed to reduce the number of diagrams.

\begin{tabular}{crrrr}
\hline process & tree & 1-loop & 2-loop & 3-loop \\
\hline$q \phi \rightarrow q \phi$ & & 1 & 23 & 696 \\
$g \phi \rightarrow g \phi$ & 1 & 8 & 218 & 6378 \\
$h \phi \rightarrow h \phi$ & & 1 & 33 & 1184 \\
\hline sum & 1 & 10 & 274 & 8258 \\
\hline
\end{tabular}

Table 1: The number of diagrams for the forward amplitudes employed in the calculation of the structure function $F_{\phi}$ to three loops. $h$ stands for the external ghost discussed in the text. 
For all external partons states in Eq. (2.13) we need to project on their physical polarizations. To that end it has proven efficient to contract the external gluon lines only with the metric tensor $-g^{\alpha \beta}$ instead of a physical projector

$$
-g^{\alpha \beta}+\frac{p^{\alpha} q^{\beta}+p^{\beta} q^{\alpha}}{p \cdot q}-\frac{p^{\alpha} p^{\beta} q^{2}}{(p \cdot q)^{2}},
$$

matching the kinematics of Eq. (2.13) with $p^{2}=0, q^{2}=-Q^{2}<0$. To compensate the additional unphysical degrees of freedom we have to consider an extra class of diagrams with external ghosts instead of external gluons, i.e., the process $h \phi \rightarrow h \phi$ listed in the third line of Table 1. In particular the resulting absence of higher tensor integrals from the numerator of Eq. (2.15) leads to a vital simplification of the calculations. For the same reason our all- $N$ computations have been performed in the Feynman gauge. As before the gauge independence has been verified for a few low values of $N$ along the lines of Refs. [23] where also the projector (2.15) has been employed for checks.

The calculation of the Mellin- $N$ projection of the forward-scattering integrals for all values of $N$ has been discussed extensively, if not exhaustively, in previous publications [6, 7, 9, 24, 37]. Also the present calculation has been carried out using version 3 of the symbolic manipulation program FORM [38] to which new features, such as an efficient database facility for huge numbers of intermediate integrals, were added in order to make such computations feasible [39]. We would like to stress again that the calculation has been set up in such a manner that checks of fixed low moments could be performed at all intermediate stages using the program MiNCER [40,41] for massless three-loop self-energy integrals. Note also that the recent computations of fixed moments of heavy-quark structure functions [42] and of the three-loop quark and gluon form factors [43] provide strong independent checks of different aspects of our calculations.

Having calculated the perturbative corrections to the individual parton contributions to $T_{\phi, k}$ in Eq. (2.12) it remains (first) to perform the renormalization of $\alpha_{s}$ and the scalar current in Eq. (2.14), and (second) to disentangle the coefficients of the Laurent expansion in terms of anomalous dimensions and coefficient functions. The second task also known as mass factorization is non-trivial, requires an explicit representation for the matrix $Z_{i k}$ in Eq. (2.6) and introduces the dependence on a specific scheme, i.e., $\overline{\mathrm{MS}}$ in our case. To that end, we set the factor $\exp \left(\varepsilon\left\{\ln (4 \pi)-\gamma_{\mathrm{e}}\right\}\right)=1$ where $\gamma_{\mathrm{e}}$ is the Euler-Mascheroni constant and choose, without loss of generality, the renormalization and mass factorization scales as $\mu^{2}=Q^{2}$.

A suitable normalization, already used in Eq. (2.11) above, at order $\alpha_{\mathrm{s}}^{0}$ implies

$$
T_{\phi, \mathrm{q}}^{(0)}=c_{\phi, \mathrm{q}}^{(0)}=0, \quad T_{\phi, \mathrm{g}}^{(0)}=c_{\phi, \mathrm{g}}^{(0)}=1 .
$$

At the first order in $\alpha_{\mathrm{s}}$ the respective forward amplitudes need to be calculated up to order $\varepsilon^{2}$ for our purposes, yielding

$$
T_{\phi, \mathrm{p}}^{(1)}=\frac{1}{\varepsilon} \gamma_{\mathrm{gp}}^{(0)}+c_{\phi, \mathrm{p}}^{(1)}+\varepsilon a_{\phi, \mathrm{p}}^{(1)}+\varepsilon^{2} b_{\phi, \mathrm{p}}^{(1)},
$$

with $\mathrm{p}=\mathrm{q}, \mathrm{g}$. Correspondingly the two-loop contributions are required up to order $\varepsilon$. These 
quantities are given by

$$
\begin{aligned}
T_{\phi, \mathrm{p}}^{(2)}= & \frac{1}{2 \varepsilon^{2}}\left\{\left(\gamma_{\mathrm{gi}}^{(0)}-\beta_{0} \delta_{\mathrm{gi}}\right) \gamma_{\mathrm{ip}}^{(0)}\right\}+\frac{1}{2 \varepsilon}\left\{\gamma_{\mathrm{gp}}^{(1)}+2 c_{\phi, \mathrm{i}}^{(1)} \gamma_{\mathrm{ip}}^{(0)}\right\} \\
& +c_{\phi, \mathrm{p}}^{(2)}+a_{\phi, \mathrm{i}}^{(1)} \gamma_{\mathrm{ip}}^{(0)}+\varepsilon\left\{a_{\phi, \mathrm{p}}^{(2)}+b_{\phi, \mathrm{i}}^{(1)} \gamma_{\mathrm{ip}}^{(0)}\right\}
\end{aligned}
$$

where $\delta_{\text {ip }}$ is the Kronecker symbol and summation over repeated indices is understood. Finally we are ready to write down the third-order coefficients $T_{\phi, k}^{(3)}$ in Eq. (2.12),

$$
\begin{aligned}
T_{\phi, \mathrm{p}}^{(3)}= & \frac{1}{6 \varepsilon^{3}}\left\{\gamma_{\mathrm{gi}}^{(0)} \gamma_{\mathrm{ik}}^{(0)} \gamma_{\mathrm{kp}}^{(0)}-3 \beta_{0} \gamma_{\mathrm{gi}}^{(0)} \gamma_{\mathrm{ip}}^{(0)}+2 \beta_{0}^{2} \gamma_{\mathrm{gp}}^{(0)}\right\} \\
& +\frac{1}{6 \varepsilon^{2}}\left\{\gamma_{\mathrm{gi}}^{(0)} \gamma_{\mathrm{ip}}^{(1)}+2 \gamma_{\mathrm{gi}}^{(1)} \gamma_{\mathrm{ip}}^{(0)}-2 \beta_{0} \gamma_{\mathrm{gp}}^{(1)}-2 \beta_{1} \gamma_{\mathrm{gp}}^{(0)}+3 c_{\phi, \mathrm{i}}^{(1)}\left(\gamma_{\mathrm{ik}}^{(0)}-\beta_{0} \delta_{\mathrm{ik}}\right) \gamma_{\mathrm{kp}}^{(0)}\right\} \\
& +\frac{1}{6 \varepsilon}\left\{2 \gamma_{\mathrm{gp}}^{(2)}+3 c_{\phi, \mathrm{i}}^{(1)} \gamma_{\mathrm{ip}}^{(1)}+6 c_{\phi, \mathrm{i}}^{(2)} \gamma_{\mathrm{ip}}^{(0)}+3 a_{\phi, \mathrm{i}}^{(1)}\left(\gamma_{\mathrm{ik}}^{(0)}-\beta_{0} \delta_{\mathrm{ik}}\right) \gamma_{\mathrm{kp}}^{(0)}\right\} \\
& +c_{\phi, \mathrm{p}}^{(3)}+\frac{1}{2} a_{\phi, \mathrm{i}}^{(1)} \gamma_{\mathrm{ip}}^{(1)}+a_{\phi, \mathrm{i}}^{(2)} \gamma_{\mathrm{ip}}^{(0)}+\frac{1}{2} b_{\phi, \mathrm{i}}^{(1)}\left(\gamma_{\mathrm{ik}}^{(0)}-\beta_{0} \delta_{\mathrm{ik}}\right) \gamma_{\mathrm{kp}}^{(0)},
\end{aligned}
$$

from which the $\mathrm{N}^{3} \mathrm{LO}$ coefficient functions $c_{\phi, \mathrm{p}}^{(3)}$ are extracted.

As mentioned above, the mass factorization in Eqs. (2.17) - 2.19) provides the splitting functions and coefficient functions in the $\overline{\mathrm{MS}}$ scheme at the scale $\mu^{2}=Q^{2}$. The full scale dependence, including the case of unequal renormalization and factorization scales, can be reconstructed from these results using, e.g., Eqs. (2.16) - (2.18) of Ref. [44].

For transformations to other schemes it is convenient to combine the present 'gluon' structure function $F_{\phi}$, recall Eq. (2.16), with a corresponding 'quark' observable such as (the flavour-singlet part of) $F_{2}$ in photon-exchange DIS [9], with $c_{2, \mathrm{q}}^{(0)}=1$ and $c_{2, \mathrm{~g}}^{(0)}=0$. Hence we define the twodimensional vector of the moments of the singlet quark and gluon distributions,

$$
q=\left(\begin{array}{c}
q_{\mathrm{s}} \\
g
\end{array}\right) \quad \text { with } \quad q_{\mathrm{s}}=\sum_{i=1}^{n_{f}}\left(q_{i}+\bar{q}_{i}\right)
$$

and the corresponding $N$-dependent quantities (recall Eq. (2.8))

$$
F=\left(\begin{array}{c}
F_{2} \\
F_{\phi}
\end{array}\right), \quad P=\left(\begin{array}{cc}
P_{\mathrm{qq}} & P_{\mathrm{qg}} \\
P_{\mathrm{gq}} & P_{\mathrm{gg}}
\end{array}\right), \quad C=\left(\begin{array}{cc}
C_{2, \mathrm{q}} & C_{2, \mathrm{~g}} \\
C_{\phi, \mathrm{q}} & C_{\phi, \mathrm{g}}
\end{array}\right)
$$

at $\mu^{2}=Q^{2}$. Any scheme transformation can now be performed by inserting a suitable (finite, no relation to Eq. (2.6) above) transformation matrix $Z$ with

$$
Z_{\mathrm{ik}}=\delta_{\mathrm{ik}}+\sum_{l=1}^{\infty} a_{\mathrm{s}}^{l} Z_{\mathrm{ik}}^{(l)}
$$

which results in transformed coefficient functions $C^{\prime}$ and parton distributions $q^{\prime}$ via

$$
F=C \cdot q=C Z^{-1} \cdot Z q=C^{\prime} \cdot q^{\prime} .
$$


The corresponding transformation of the splitting functions reads

$$
P^{\prime}=P+\left(\beta \frac{d Z}{d a_{\mathrm{s}}}+[Z, P]\right) Z^{-1}
$$

where $\beta$ represents the right-hand-side of Eq. (2.10), and $[Z, P]$ the standard commutator of the matrices $Z$ and $P$. To order $\alpha_{\mathrm{s}}^{4}\left(\mathrm{~N}^{3} \mathrm{LO}\right)$ the insertion of the perturbative expansions thus leads to

$$
\begin{aligned}
P^{\prime}= & a_{\mathrm{s}} P_{0} \\
+ & a_{\mathrm{s}}^{2}\left(P^{(1)}+\left[Z^{(1)}, P^{(0)}\right]-\beta_{0} Z^{(1)}\right) \\
& +a_{\mathrm{s}}^{3}\left(P^{(2)}+\left[Z^{(2)}, P^{(0)}\right]+\left[Z^{(1)}, P^{(1)}\right]-\left[Z^{(1)}, P^{(0)}\right] Z^{(1)}+\beta_{0}\left(\left\{Z^{(1)}\right\}^{2}-2 Z^{(2)}\right)-\beta_{1} Z^{(1)}\right) \\
& +a_{\mathrm{s}}^{4}\left(P^{(3)}+\left[Z^{(3)}, P^{(0)}\right]+\left[Z^{(2)}, P^{(1)}\right]+\left[Z^{(1)}, P^{(2)}\right]-\left(\left[Z^{(2)}, P^{(0)}\right]+\left[Z^{(1)}, P^{(1)}\right]\right) Z^{(1)}\right. \\
& \quad+\left[Z^{(1)}, P^{(0)}\right]\left(\left\{Z^{(1)}\right\}^{2}-2 Z^{(2)}\right)-\beta_{0}\left(\left\{Z^{(1)}\right\}^{3}-Z^{(1)} Z^{(2)}-2 Z^{(2)} Z^{(1)}+3 Z^{(3)}\right) \\
& \left.+\beta_{1}\left(\left\{Z^{(1)}\right\}^{2}-2 Z^{(2)}\right)-\beta_{2} Z^{(1)}\right)+o\left(a_{\mathrm{s}}^{5}\right) .
\end{aligned}
$$

In order to transfer the momentum sum rule, $q_{\mathrm{s}}+g=1$ at $N=2$ for all scales, from $\overline{\mathrm{MS}}$ to the transformed parton densities $q^{\prime}$ in Eq. (2.23), the coefficients in Eq. (2.22) need to satisfy the relations

$$
Z_{\mathrm{qq}}^{(l)}(N=2)+Z_{\mathrm{gq}}^{(l)}(N=2)=0, \quad Z_{\mathrm{qg}}^{(l)}(N=2)+Z_{\mathrm{gg}}^{(l)}(N=2)=0 .
$$

Eqs. (2.22) - (2.25) can be employed to transform to the physical evolution equations for the system $F=\left(F_{2}, F_{\phi}\right)$ by choosing

$$
\left(\begin{array}{cc}
Z_{\mathrm{qq}}^{(l)} & Z_{\mathrm{qg}}^{(l)} \\
Z_{\mathrm{gq}}^{(l)} & Z_{\mathrm{gg}}^{(l)}
\end{array}\right)=\left(\begin{array}{cc}
c_{2, \mathrm{q}}^{(l)} & c_{2, \mathrm{~g}}^{(l)} \\
c_{\phi, \mathrm{q}}^{(l)} & c_{\phi, \mathrm{g}}^{(l)}
\end{array}\right)
$$

in Eq. (2.22). This leads to

$$
\frac{d}{d \ln Q^{2}} F=K F \equiv \sum_{l=0}^{\infty} a_{\mathrm{s}}^{l+1}\left(\begin{array}{cc}
K_{22}^{(l)} & K_{2 \phi}^{(l)} \\
K_{\phi 2}^{(l)} & K_{\phi \phi}^{(l)}
\end{array}\right) \cdot\left(\begin{array}{c}
F_{2} \\
F_{\phi}
\end{array}\right)
$$

where the matrix elements of the physical evolution kernel $K$ are given by Eq. (2.25) after inserting Eq. (2.27). As far as we know this transformation has first been suggested (at NLO) in Ref. [16]. The above relations refer to the choice $\mu^{2}=Q^{2}$ of the renormalization scale - the mass factorization scheme and scale have now been eliminated from the problem. From these results the physical kernel at $\mu^{2} \neq Q^{2}$ (or in other renormalization schemes) can be reconstructed in the usual way. As we shall see below, Eq. (2.27) does not represent a 'normal' scheme transformation of the quark and gluon distributions, since the $\overline{\mathrm{MS}}$ coefficient functions for $F_{2}$ and $F_{\phi}$ on the right-hand-side do not fulfill the momentum sum rule constraints 2.26. 


\section{Coefficient functions for Higgs-exchange DIS}

In this section we present and discuss the $x$-space results, obtained from our above $N$-space calculations by a by now standard inverse Mellin transformation [24,45], for the previously unpublished $\overline{\mathrm{MS}}$-scheme coefficient functions $c_{\phi, \mathrm{q}}^{(l)}$ and $c_{\phi, \mathrm{g}}^{(l)}, l=1,2,3$, at the standard choice $\mu^{2}=Q^{2}$ of the renormalization and factorization scale.

We express our results in terms of the harmonic polylogarithms $H_{m_{1}, \ldots, m_{w}}(x)$ with $m_{j}=0, \pm 1$. Our notation for these functions follows Ref. [45] to which the reader is referred for a detailed discussion. For completeness we recall the basic definitions: The lowest-weight $(w=1)$ functions $H_{m}(x)$ are given by

$$
H_{0}(x)=\ln x, \quad H_{ \pm 1}(x)=\mp \ln (1 \mp x) .
$$

The higher-weight $(w \geq 2)$ functions are recursively defined as

$$
H_{m_{1}, \ldots, m_{w}}(x)=\left\{\begin{array}{cl}
\frac{1}{w !} \ln ^{w} x, & \text { if } m_{1}, \ldots, m_{w}=0, \ldots, 0 \\
\int_{0}^{x} d z f_{m_{1}}(z) H_{m_{2}, \ldots, m_{w}}(z), & \text { else }
\end{array}\right.
$$

with

$$
f_{0}(x)=\frac{1}{x}, \quad f_{ \pm 1}(x)=\frac{1}{1 \mp x} .
$$

For chains of indices zero we employ the abbreviated notation

$$
H_{\underbrace{0, \ldots, 0}_{m}}, \pm 1, \underbrace{0, \ldots, 0}_{n}, \pm 1, \ldots(x)=H_{ \pm(m+1), \pm(n+1), \ldots}(x)
$$

and usually suppress the argument $x$.

The $l$-th order coefficient functions involve harmonic polylogarithms up to weight $2 l-1$. Hence only the results up to two loops, with $w \leq 3$, can be expressed in terms of standard polylogarithms. A complete list can be found in appendix A of Ref. [24]. A FORTRAN program for the harmonic polylogarithms including weight $w=4$ has been published in Ref. [46]. Its extension to $w=5$, required for the third-order coefficient functions, is also available [47].

In this notation the first-order coefficient functions are given by

$$
\begin{aligned}
c_{\phi, \mathrm{q}}^{(1)}(x)= & C_{F}\left(2 p_{\mathrm{gq}}(x)\left(-\mathrm{H}_{0}-\mathrm{H}_{1}\right)-3 x^{-1}+2 x\right), \\
c_{\phi, \mathrm{g}}^{(1)}(x)= & C_{A}\left(4 p_{\mathrm{gg}}(x)\left(-\mathrm{H}_{0}-\mathrm{H}_{1}\right)-11 / 3\left((1-x)^{-1}+x^{-1}\right)+\left(67 / 9-4 \zeta_{2}\right) \delta(1-x)\right) \\
& +n_{f}\left(2 / 3\left((1-x)^{-1}+x^{-1}\right)-10 / 9 \delta(1-x)\right) .
\end{aligned}
$$

Here and below $\zeta_{n}$ represents the Riemann zeta-function, and as above $n_{f}$ denotes the number of effectively massless flavours. $C_{A}$ and $C_{F}$ are the usual QCD colour factors specified below Eq. (2.10). Finally we have employed the abbreviations 


$$
\begin{aligned}
& p_{\mathrm{gq}}(x)=2 x^{-1}-2+x \\
& p_{\mathrm{gg}}(x)=(1-x)^{-1}+x^{-1}-2+x-x^{2}
\end{aligned}
$$

The corresponding two-loop (NLO) coefficient functions read

$$
\begin{aligned}
c_{\phi, \mathrm{q}}^{(2)}(x) & =C_{F}^{2}\left(4 p_{\mathrm{gq}}(x)\left(-6 \mathrm{H}_{1,1}-2 \mathrm{H}_{1,1,0}-\mathrm{H}_{1,1,1}-2 \mathrm{H}_{1,2}-2 \mathrm{H}_{2,0}-2 \mathrm{H}_{2,1}-2 \mathrm{H}_{3}\right)\right. \\
& +8 p_{\mathrm{gq}}(-x)\left(-\mathrm{H}_{-1} \zeta_{2}-2 \mathrm{H}_{-1,-1,0}+\mathrm{H}_{-1,0,0}-\mathrm{H}_{0} \zeta_{2}\right)-16(1+x) \mathrm{H}_{-1,0} \\
& -4 \zeta_{3}\left(2+8 x^{-1}+3 x\right)+2(2-x)\left(5 \mathrm{H}_{0,0,0}+2 \mathrm{H}_{2,0}+2 \mathrm{H}_{2,1}+4 \mathrm{H}_{3}\right) \\
& +4\left(5-9 x^{-1}-6 x\right) \mathrm{H}_{2}-4 \zeta_{2}\left(5-9 x^{-1}-2 x\right)-8 \zeta_{2}(6-x) \mathrm{H}_{0}-(12-x) \mathrm{H}_{0,0} \\
& +\left(15-10 x^{-1}+18 x\right) \mathrm{H}_{0}+2\left(16-18 x^{-1}-7 x\right) \mathrm{H}_{1,0}+\left(56-64 x^{-1}+3 x\right) \mathrm{H}_{1} \\
& \left.+1 / 2\left(208-59 x^{-1}-30 x\right)-32 \mathrm{H}_{-2,0}-4 \mathrm{H}_{1,1}\right) \\
+ & C_{A} C_{F}\left(20 p_{\mathrm{gq}}(x)\left(\zeta_{2} \mathrm{H}_{1}-\mathrm{H}_{1,0,0}-\mathrm{H}_{1,1,0}-\mathrm{H}_{1,1,1}-\mathrm{H}_{1,2}\right)\right. \\
& +4 p_{\mathrm{gq}}(-x)\left(-2 \mathrm{H}_{-1} \zeta_{2}+3 \mathrm{H}_{-1,0,0}+2 \mathrm{H}_{-1,2}\right)+16\left(1+2 x^{-1}+2 x\right)\left(\zeta_{2} \mathrm{H}_{0}-\mathrm{H}_{3}\right) \\
& +4\left(2-10 x^{-1}-7 x\right) \mathrm{H}_{2,1}-4\left(10+4 x^{-1}+7 x\right) \mathrm{H}_{0,0,0}-4 \zeta_{3}\left(16-18 x^{-1}-11 x\right) \\
& +4 / 3\left(24+22 x^{-1}+9 x+4 x^{2}\right) \mathrm{H}_{-1,0}+4 / 3\left(46-53 x^{-1}+x+4 x^{2}\right) \mathrm{H}_{1,0} \\
& -1 / 9\left(60+1362 x^{-1}+513 x+352 x^{2}\right) \mathrm{H}_{0}+2 / 3\left(70-84 x^{-1}+13 x+8 x^{2}\right) \mathrm{H}_{1,1} \\
& -4 / 3 \zeta_{2}\left(88-53 x^{-1}+7 x+12 x^{2}\right)+4 / 3\left(88-31 x^{-1}+16 x+12 x^{2}\right) \mathrm{H}_{2} \\
& +1 / 9\left(242-288 x^{-1}-127 x-176 x^{2}\right) \mathrm{H}_{1}+2 / 3\left(268-106 x^{-1}+13 x+32 x^{2}\right) \mathrm{H}_{0,0} \\
& \left.+1 / 54\left(6172-13457 x^{-1}+76 x+1216 x^{2}\right)-8\left(4 x^{-1}+3 x\right) \mathrm{H}_{2,0}-16 \mathrm{H}_{-2,0}\right) \\
+ & C_{F} n_{f}\left(2 / 9 p_{\mathrm{gq}}(x)\left(24 \mathrm{H}_{0,0}+29 \mathrm{H}_{1}+12 \mathrm{H}_{1,0}+6 \mathrm{H}_{1,1}+12 \mathrm{H}_{2}\right)+8 / 3 \zeta_{2}\left(2-2 x^{-1}-x\right)\right. \\
& \left.-2 / 3\left(26-32 x^{-1}-11 x\right) \mathrm{H}_{0}-1 / 27\left(332-737 x^{-1}-28 x\right)\right)
\end{aligned}
$$

and

$$
\begin{aligned}
c_{\phi, \mathrm{g}}^{(2)}(x) & =C_{A}^{2}\left(p _ { \mathrm { gg } } ( x ) \left(-2570 / 27+68 \zeta_{3}+176 / 3 \zeta_{2}+24 \mathrm{H}_{-2,0}-778 / 9 \mathrm{H}_{0}+56 \mathrm{H}_{0} \zeta_{2}\right.\right. \\
& -44 \mathrm{H}_{0,0}-28 \mathrm{H}_{0,0,0}-778 / 9 \mathrm{H}_{1}+40 \mathrm{H}_{1} \zeta_{2}-176 / 3 \mathrm{H}_{1,0}-40 \mathrm{H}_{1,0,0}-176 / 3 \mathrm{H}_{1,1} \\
& \left.-56 \mathrm{H}_{1,1,0}-48 \mathrm{H}_{1,1,1}-56 \mathrm{H}_{1,2}-176 / 3 \mathrm{H}_{2}-48 \mathrm{H}_{2,0}-56 \mathrm{H}_{2,1}-56 \mathrm{H}_{3}\right) \\
& +4 p_{\mathrm{gg}}(-x)\left(7 \zeta_{3}+6 \mathrm{H}_{-2,0}-8 \mathrm{H}_{-1} \zeta_{2}-8 \mathrm{H}_{-1,-1,0}+10 \mathrm{H}_{-1,0,0}+4 \mathrm{H}_{-1,2}+2 \mathrm{H}_{0} \zeta_{2}\right. \\
& \left.-3 \mathrm{H}_{0,0,0}-2 \mathrm{H}_{3}\right)+16(1+x)\left(2 \zeta_{3}+4 \mathrm{H}_{0} \zeta_{2}-5 \mathrm{H}_{0,0,0}-2 \mathrm{H}_{2,0}-2 \mathrm{H}_{2,1}-4 \mathrm{H}_{3}\right) \\
& +8 / 3\left(6+11 x^{-1}+6 x+11 x^{2}\right) \mathrm{H}_{-1,0}+1 / 27\left(10-5659 x^{-1}-1916 x+3177 x^{2}\right) \\
& +4 / 3\left(14-44 x^{-1}-25 x+33 x^{2}\right)\left(\mathrm{H}_{1,0}+\mathrm{H}_{1,1}\right)-4 / 3 \zeta_{2}\left(45-44 x^{-1}-24 x+77 x^{2}\right) \\
& +4 / 3\left(45-22 x^{-1}-12 x+77 x^{2}\right) \mathrm{H}_{2}+2 / 3\left(171-44 x^{-1}-33 x+220 x^{2}\right) \mathrm{H}_{0,0} \\
& -2 / 9\left(182+121 x^{-1}-58 x\right) \mathrm{H}_{1}-1 / 9\left(1107+778 x^{-1}+699 x+536 x^{2}\right) \mathrm{H}_{0} \\
& \left.+\left(30425 / 162-242 / 3 \zeta_{3}-778 / 9 \zeta_{2}+101 / 5 \zeta_{2}^{2}\right) \delta(1-x)\right)
\end{aligned}
$$




$$
\begin{aligned}
+ & C_{A} n_{f}\left(p _ { \mathrm { gg } } ( x ) \left(224 / 9-32 / 3 \zeta_{2}+56 / 3 \mathrm{H}_{0}+8 \mathrm{H}_{0,0}+56 / 3 \mathrm{H}_{1}+32 / 3\left(\mathrm{H}_{1,0}+\mathrm{H}_{1,1}\right)\right.\right. \\
& \left.+32 / 3 \mathrm{H}_{2}\right)+4(1+x) \mathrm{H}_{0,0}+8 / 3\left(2-x+x^{2}\right)\left(\mathrm{H}_{1,0}+\mathrm{H}_{1,1}\right)+8 / 3\left(3+x^{2}\right)\left(\mathrm{H}_{2}-\zeta_{2}\right) \\
& +2 / 9\left(40+48 x^{-1}+7 x-4 x^{2}\right) \mathrm{H}_{1}+2 / 9\left(47+48 x^{-1}+14 x-4 x^{2}\right) \mathrm{H}_{0} \\
& \left.+2 / 27\left(389+320 x^{-1}-46 x+59 x^{2}\right)-\left(4112 / 81+28 / 3 \zeta_{3}-56 / 3 \zeta_{2}\right) \delta(1-x)\right) \\
+ & C_{F} n_{f}\left(2 p_{\mathrm{gg}}(x)+4(1+x)\left(-2 \zeta_{3}-4 \mathrm{H}_{0} \zeta_{2}+5 \mathrm{H}_{0,0,0}+2 \mathrm{H}_{2,0}+2 \mathrm{H}_{2,1}+4 \mathrm{H}_{3}\right)\right. \\
& +4 / 3\left(3+4 x^{-1}-3 x-4 x^{2}\right)\left(\mathrm{H}_{1,0}+\mathrm{H}_{1,1}\right)+4 / 3\left(9+4 x^{-1}+6 x-4 x^{2}\right)\left(\mathrm{H}_{2}-\zeta_{2}\right) \\
& +2 / 3\left(21+8 x^{-1}+15 x-8 x^{2}\right) \mathrm{H}_{0,0}+2 / 9\left(93+32 x^{-1}-57 x-68 x^{2}\right) \mathrm{H}_{1} \\
& +2 / 9\left(129+32 x^{-1}-3 x-68 x^{2}\right) \mathrm{H}_{0}+1 / 27\left(1671+307 x^{-1}-1212 x-442 x^{2}\right) \\
& \left.-\left(63 / 2-24 \zeta_{3}\right) \delta(1-x)\right) \\
+ & n_{f}^{2}\left(8 / 27 p_{\mathrm{gg}}(x)\left(-5-3 \mathrm{H}_{0}-3 \mathrm{H}_{1}\right)+8 / 27\left(2-x+x^{2}\right)\left(-5-3 \mathrm{H}_{0}-3 \mathrm{H}_{1}\right)\right. \\
& \left.+\left(100 / 81-8 / 9 \zeta_{2}\right) \delta(1-x)\right) .
\end{aligned}
$$

As mentioned in the introduction, the above results have also been derived, in a quite different and completely independent manner, in Ref. [30]. As their counterparts for photon- and $W$-exchange DIS presented in Refs. [9, 10], the full third-order expressions, not obtained by any other group so far, for the present Higgs-exchange coefficient functions are exceedingly long. Therefore these expressions are deferred to Appendix A.

Before we illustrate the numerical size and perturbative stability of these coefficient functions, it is instructive to discuss their behaviour close to the endpoints $x=1$ and $x=0$. For this we will use to abbreviations

$$
x_{1}=1-x, \quad L_{0}=\ln x, \quad L_{1}=\ln x_{1}, \quad \mathcal{D}_{k}=\left[x_{1}^{-1} L_{1}^{k}\right]_{+} .
$$

As usual, the +-distributions are defined by

$$
\int_{0}^{1} d x a(x)_{+} f(x)=\int_{0}^{1} d x a(x)\{f(x)-f(1)\}
$$

for regular functions $f(x)$. It is understood that all $1 /(1-x)$ poles in Eqs. (3.6), (3.9) and (A.2) have to be read as +-distributions.

The leading large- $x$ contributions to the gluon coefficient functions $c_{\phi, g}^{(n)}(x)$ are given by softgluon emission contributions $\mathcal{D}_{k}$ with $k=0, \ldots, 2 n-1$. At the first order these and all other endpoint contributions can be read off directly from Eqs. (3.5) and (3.6) with the help of Eq. (3.1). The +-distribution coefficients of the two-loop gluon coefficient function read

$$
\begin{aligned}
& \left.c_{\phi, \mathrm{g}}^{(2)}\right|_{\mathcal{D}_{3}}=8 C_{A}^{2}, \\
& \left.c_{\phi, \mathrm{g}}^{(2)}\right|_{\mathcal{D}_{2}}=-\frac{88}{3} C_{A}^{2}+\frac{16}{3} C_{A} n_{f},
\end{aligned}
$$




$$
\begin{aligned}
& \left.c_{\phi, \mathrm{g}}^{(2)}\right|_{\mathcal{D}_{1}}=C_{A}^{2}\left(\frac{778}{9}-40 \zeta_{2}\right)-\frac{56}{3} C_{A} n_{f}+\frac{8}{9} n_{f}^{2}, \\
& \left.c_{\phi, \mathrm{g}}^{(2)}\right|_{\mathcal{D}_{0}}=-C_{A}^{2}\left(\frac{2570}{27}-32 \zeta_{3}-\frac{176}{3} \zeta_{2}\right)+C_{A} n_{f}\left(\frac{224}{9}-\frac{32}{3} \zeta_{2}\right)+2 C_{F} n_{f}-\frac{40}{27} n_{f}^{2},
\end{aligned}
$$

and the corresponding contributions at the third-order are given by

$$
\begin{aligned}
& \left.c_{\phi, \mathrm{g}}^{(3)}\right|_{\mathcal{D}_{5}}=8 C_{A}^{3} \\
& \left.c_{\phi, \mathrm{g}}^{(3)}\right|_{\mathcal{D}_{4}}=-\frac{550}{9} C_{A}^{3}+\frac{100}{9} C_{A}^{2} n_{f} \\
& \left.c_{\phi, \mathrm{g}}^{(3)}\right|_{\mathcal{D}_{3}}=C_{A}^{3}\left(340-128 \zeta_{2}\right)-\frac{256}{3} C_{A}^{2} n_{f}+\frac{16}{3} C_{A} n_{f}^{2}, \\
& \left.c_{\phi, \mathrm{g}}^{(3)}\right|_{\mathcal{D}_{2}}=-C_{A}^{3}\left(\frac{9623}{9}-\frac{1892}{3} \zeta_{2}-256 \zeta_{3}\right)+C_{A}^{2} n_{f}\left(\frac{3106}{9}-\frac{344}{3} \zeta_{2}\right) \\
& +16 C_{F} C_{A} n_{f}-\frac{292}{9} C_{A} n_{f}^{2}+\frac{8}{9} n_{f}^{3} \\
& \left.c_{\phi, \mathrm{g}}^{(3)}\right|_{\mathcal{D}_{1}}=C_{A}^{3}\left(\frac{192268}{81}-\frac{16268}{9} \zeta_{2}-\frac{2816}{3} \zeta_{3}+\frac{1316}{5} \zeta_{2}^{2}\right) \\
& -C_{A}^{2} n_{f}\left(\frac{67730}{81}-\frac{4192}{9} \zeta_{2}-\frac{128}{3} \zeta_{3}\right)-C_{F} C_{A} n_{f}\left(\frac{598}{3}-128 \zeta_{3}\right) \\
& +C_{A} n_{f}^{2}\left(\frac{6652}{81}-\frac{272}{9} \zeta_{2}\right)+\frac{20}{3} C_{F} n_{f}^{2}-\frac{80}{27} n_{f}^{3} \\
& \left.c_{\phi, \mathrm{g}}^{(3)}\right|_{\mathcal{D}_{0}}=-C_{A}^{3}\left(\frac{1616486}{729}-\frac{169910}{81} \zeta_{2}-\frac{40454}{27} \zeta_{3}+\frac{7931}{15} \zeta_{2}^{2}+\frac{1280}{3} \zeta_{2} \zeta_{3}-80 \zeta_{5}\right) \\
& +C_{A}^{2} n_{f}\left(\frac{1234307}{1458}-\frac{55388}{81} \zeta_{2}-\frac{1816}{9} \zeta_{3}+\frac{1538}{15} \zeta_{2}^{2}\right)-C_{F}^{2} n_{f} \\
& +C_{F} C_{A} n_{f}\left(\frac{7810}{27}-32 \zeta_{2}-\frac{1624}{9} \zeta_{3}-\frac{32}{5} \zeta_{2}^{2}\right)-C_{F} n_{f}^{2}\left(\frac{350}{9}-\frac{80}{3} \zeta_{3}\right) \\
& -C_{A} n_{f}^{2}\left(\frac{138493}{1458}-\frac{584}{9} \zeta_{2}+\frac{152}{27} \zeta_{3}\right)+n_{f}^{3}\left(\frac{200}{81}-\frac{16}{9} \zeta_{2}\right) \text {. }
\end{aligned}
$$

Together with the $\delta(1-x)$ contributions arising from soft-gluon emissions and virtual corrections - which can be read off directly from Eqs. (3.6), (3.9) and (A.2) - these results have be employed in Ref. [25] to obtain the soft-gluon exponentiation of $C_{\phi, g}$ to the next-to-next-to-next-toleading logarithmic $\left(\mathrm{N}^{3} \mathrm{LL}\right)$ accuracy. Consequently the highest seven +-distributions are known at the fourth and all higher orders in $\alpha_{\mathrm{s}}$, which the exception of the (almost certainly numerically irrelevant) contribution of the four-loop cusp anomalous dimension to the coefficient of $\alpha_{\mathrm{s}}^{n} \mathcal{D}_{2 n-7}$.

As discussed in Refs. [26, 48], the computation of the forward amplitudes $T_{2, \mathrm{q}}^{(l)}$ and $T_{\phi, \mathrm{g}}^{(l)}$ in photon- and Higgs-exchange DIS to order $\alpha_{\mathrm{s}}^{l} \varepsilon^{3-l}$ with $l \leq 3$, in the above soft + virtual limit, facilitates the determination of all $1 / \varepsilon$ pole terms of the three-loop $\gamma^{*} q q$ and $H g g$ form factors 
(the latter, of course, in the heavy-top limit). The corresponding results, including the additional $\varepsilon^{0} n_{f}$ contributions in the $\gamma^{*} q q$ case, have been verified recently in direct calculations of these form factors [43], thus providing a check of Eqs. (3.16) - (3.21) and the $\delta(1-x)$ in Eq. (A.2) and the corresponding results in Refs. [6,7,9] including, in particular, the full results for the three-loop quark and gluon cusp anomalous dimensions. Those results, in turn, involve a considerable part of the three-loop forward-scattering diagrams and integrals entering the complete (all- $x$ ) calculations of the third-order splitting functions and coefficient functions.

Returning to the large- $x$ behaviour of the gluon coefficient functions $c_{\phi, \mathrm{g}}^{(n)}(x)$ we note that, as Eqs. (3.12) - (3.14) and (3.16) - (3.18) above, the coefficients of the highest three subleading $\ln ^{k}(1-x)$ terms, $k=3,2,1$ for $c_{\phi, \mathrm{g}}^{(2)}(x)$ and $k=5,4,3$ for $c_{\phi, \mathrm{g}}^{(2)}(x)$, do not include contributions with the colour factor $C_{F}$, i.e., gluon emission from quarks. Hence they are guaranteed to originate from 'non-singlet like' diagrams with a (modulo self-energy insertions) unbroken gluon line connecting the incoming gluon to the scalar $\phi$. Consequently non-singlet considerations and structures hold for these contributions, cf. Refs. $[15,19,29]$, and these subleading logarithms can be predicted to all orders $n$ along the lines of Ref. [20].

We now turn to the quark coefficient functions $c_{\phi, \mathrm{q}}^{(n)}(x)$ where the integrable logarithms $L_{1}^{k} \equiv$ $\ln ^{k}(1-x)$ with $k=1, \ldots, 2 n-1$ form the leading large- $x$ terms. Again the first-order coefficient is obvious from Eq. (3.5), and the second-order contributions are given by

$$
\begin{aligned}
& \left.c_{\phi, \mathrm{q}}^{(2)}\right|_{L_{1}^{3}}=\frac{10}{3} C_{F} C_{A}+\frac{2}{3} C_{F}^{2}, \\
& \left.c_{\phi, \mathrm{q}}^{(2)}\right|_{L_{1}^{2}}=\frac{7}{3} C_{F} C_{A}-14 C_{F}^{2}+\frac{2}{3} C_{F} n_{f}, \\
& \left.c_{\phi, \mathrm{q}}^{(2)}\right|_{L_{1}}=C_{F} C_{A}\left(\frac{349}{9}-20 \zeta_{2}\right)+5 C_{F}^{2}-\frac{58}{9} C_{F} n_{f} .
\end{aligned}
$$

The corresponding three-loop results read

$$
\begin{aligned}
\left.c_{\phi, \mathrm{q}}^{(3)}\right|_{L_{1}^{5}}= & \frac{10}{3} C_{F} C_{A}^{2}+\frac{2}{3} C_{F}^{3}, \\
\left.c_{\phi, \mathrm{q}}^{(3)}\right|_{L_{1}^{4}}= & -\frac{61}{54} C_{F} C_{A}^{2}-\frac{442}{27} C_{F}^{2} C_{A}-\frac{157}{18} C_{F}^{3}+\frac{47}{27} C_{F} C_{A} n_{f}+\frac{13}{27} C_{F}^{2} n_{f}, \\
\left.c_{\phi, \mathrm{q}}^{(3)}\right|_{L_{1}^{3}}= & C_{F} C_{A}^{2}\left(\frac{5894}{81}-\frac{368}{9} \zeta_{2}\right)+C_{F}^{2} C_{A}\left(\frac{5161}{81}-\frac{296}{9} \zeta_{2}\right)+C_{F}^{3}\left(\frac{74}{3}+\frac{88}{9} \zeta_{2}\right) \\
& -\frac{1148}{81} C_{F} C_{A} n_{f}-\frac{1018}{81} C_{F}^{2} n_{f}+\frac{16}{27} C_{F} n_{f}^{2}, \\
\left.c_{\phi, \mathrm{q}}^{(3)}\right|_{L_{1}^{2}}= & -C_{F} C_{A}^{2}\left(\frac{8213}{81}-\frac{296}{9} \zeta_{2}-84 \zeta_{3}\right)-C_{F}^{2} C_{A}\left(\frac{40486}{81}-\frac{608}{3} \zeta_{2}-\frac{316}{3} \zeta_{3}\right) \\
& +C_{F}^{3}\left(\frac{295}{3}+\frac{104}{3} \zeta_{2}-\frac{184}{3} \zeta_{3}\right)+C_{F} C_{A} n_{f}\left(\frac{3236}{81}-\frac{248}{9} \zeta_{2}\right) \\
& +C_{F}^{2} n_{f}\left(\frac{6616}{81}+\frac{4}{3} \zeta_{2}\right)-\frac{104}{27} n_{f}^{2} C_{F},
\end{aligned}
$$




$$
\begin{aligned}
\left.c_{\phi, \mathrm{q}}^{(3)}\right|_{L_{1}}= & C_{F} C_{A}^{2}\left(\frac{227065}{243}-\frac{17384}{27} \zeta_{2}-\frac{590}{3} \zeta_{3}+\frac{368}{5} \zeta_{2}^{2}\right)+C_{F}^{2} C_{A}\left(\frac{524873}{972}\right. \\
& \left.-\frac{638}{27} \zeta_{2}-\frac{2954}{9} \zeta_{3}+\frac{2674}{15} \zeta_{2}^{2}\right)-C_{F}^{3}\left(\frac{2927}{12}+170 \zeta_{2}-\frac{328}{3} \zeta_{3}+\frac{1804}{15} \zeta_{2}^{2}\right) \\
& -C_{F} C_{A} n_{f}\left(\frac{75052}{243}-\frac{3260}{27} \zeta_{2}-\frac{52}{3} \zeta_{3}\right)-C_{F}^{2} n_{f}\left(\frac{70747}{486}-\frac{812}{27} \zeta_{2}-\frac{272}{9} \zeta_{3}\right) \\
& +C_{F} n_{f}^{2}\left(\frac{544}{27}-\frac{40}{9} \zeta_{2}\right) .
\end{aligned}
$$

The two-loop coefficients 3.22 - 3.24) contribute to the large- $x$ behaviour of the NNLO physical kernel $K_{\phi 2}^{(2)}(x)$, recall Eqs. (2.27) and (2.28) above, which will be discussed in the next section. The third-order results (3.25) - 3.27) will enter our large- $x$ predictions for the four-loop splitting function $P_{\mathrm{gq}}^{(3)}(x)$ in Section 5 .

At small $x$ both the coefficient functions $c_{\phi, p}^{(n)}(x), \mathrm{p}=\mathrm{q}, \mathrm{g}$, show a double-logarithmic enhancement, i.e., terms $x^{-1} \ln ^{k} x$ contribute with $k=0, \ldots, 2 n-1$. The second-order coefficients are

$$
\begin{aligned}
\left.x c_{\phi, \mathrm{q}}^{(2)}\right|_{L_{0}^{3}}= & -\frac{8}{3} C_{F} C_{A}, \\
\left.x c_{\phi, \mathrm{q}}^{(2)}\right|_{L_{0}^{2}}= & -\frac{106}{3} C_{F} C_{A}+\frac{16}{3} C_{F} n_{f}, \\
\left.x c_{\phi, \mathrm{q}}^{(2)}\right|_{L_{0}}= & -C_{F} C_{A}\left(\frac{454}{3}-32 \zeta_{2}\right)-C_{F}^{2}\left(10-16 \zeta_{2}\right)+\frac{64}{3} C_{F} n_{f}, \\
\left.x c_{\phi, \mathrm{q}}^{(2)}\right|_{L_{0}^{0}}= & -C_{F} C_{A}\left(\frac{13457}{54}-\frac{212}{3} \zeta_{2}-72 \zeta_{3}\right)-C_{F}^{2}\left(\frac{59}{2}-36 \zeta_{2}+32 \zeta_{3}\right) \\
& +C_{F} n_{f}\left(\frac{737}{27}-\frac{16}{3} \zeta_{2}\right)
\end{aligned}
$$

and

$$
\begin{aligned}
\left.x c_{\phi, \mathrm{g}}^{(2)}\right|_{L_{0}^{3}}= & -\frac{8}{3} C_{A}^{2}, \\
\left.x c_{\phi, \mathrm{g}}^{(2)}\right|_{L_{0}^{2}}= & -\frac{110}{3} C_{A}^{2}+4 C_{A} n_{f}+\frac{8}{3} C_{F} n_{f}, \\
\left.x c_{\phi, \mathrm{g}}^{(2)}\right|_{L_{0}}= & -C_{A}^{2}\left(\frac{1556}{9}-48 \zeta_{2}\right)+\frac{88}{3} C_{A} n_{f}+\frac{64}{9} C_{F} n_{f}-\frac{8}{9} n_{f}^{2}, \\
\left.x c_{\phi, \mathrm{g}}^{(2)}\right|_{L_{0}^{0}}= & -C_{A}^{2}\left(\frac{2743}{9}-\frac{352}{3} \zeta_{2}-40 \zeta_{3}\right)+C_{A} n_{f}\left(\frac{1312}{27}-\frac{32}{3} \zeta_{2}\right) \\
& +C_{F} n_{f}\left(\frac{361}{27}-\frac{16}{3} \zeta_{2}\right)-\frac{40}{27} n_{f}^{2} .
\end{aligned}
$$

Their three-loop counterpart are found to be

$$
\left.x c_{\phi, \mathrm{q}}^{(3)}\right|_{L_{0}^{5}}=-\frac{8}{15} C_{F} C_{A}^{2},
$$




$$
\begin{aligned}
& \left.x c_{\phi, \mathrm{q}}^{(3)}\right|_{L_{0}^{4}}=-24 C_{F} C_{A}^{2}+\frac{28}{9} C_{F} C_{A} n_{f}+\frac{8}{9} C_{F}^{2} n_{f}, \\
& \left.x c_{\phi, \mathrm{q}}^{(3)}\right|_{L_{0}^{3}}=-C_{F} C_{A}^{2}\left(\frac{1010}{3}-32 \zeta_{2}\right)-C_{F}^{2} C_{A}\left(\frac{20}{3}-\frac{32}{3} \zeta_{2}\right)+\frac{2348}{27} n_{f} C_{F} C_{A} \\
& +\frac{40}{27} C_{F}^{2} n_{f}-\frac{16}{3} C_{F} n_{f}^{2} \\
& \left.x c_{\phi, \mathrm{q}}^{(3)}\right|_{L_{0}^{2}}=-C_{F} C_{A}^{2}\left(\frac{16793}{9}-\frac{1288}{3} \zeta_{2}-\frac{208}{3} \zeta_{3}\right)-C_{F}^{2} C_{A}\left(\frac{833}{3}-\frac{992}{3} \zeta_{2}+16 \zeta_{3}\right) \\
& +C_{F}^{3}\left(52-48 \zeta_{2}+32 \zeta_{3}\right)+C_{F} C_{A} n_{f}\left(546-48 \zeta_{2}\right) \\
& +C_{F}^{2} n_{f}\left(\frac{1654}{27}-64 \zeta_{2}\right)-\frac{964}{27} n_{f}^{2} C_{F} \\
& \left.x c_{\phi, \mathrm{q}}^{(3)}\right|_{L_{0}}=-C_{F} C_{A}^{2}\left(\frac{353878}{81}-\frac{49580}{27} \zeta_{2}-\frac{15104}{9} \zeta_{3}+\frac{5584}{15} \zeta_{2}^{2}\right) \\
& -C_{F}^{2} C_{A}\left(\frac{14701}{9}-\frac{15052}{9} \zeta_{2}+\frac{1448}{3} \zeta_{3}+\frac{208}{5} \zeta_{2}^{2}\right)+C_{F}^{3}\left(450-224 \zeta_{2}\right. \\
& \left.+144 \zeta_{3}-128 \zeta_{2}^{2}\right)+C_{F} C_{A} n_{f}\left(\frac{122170}{81}-\frac{10600}{27} \zeta_{2}-\frac{400}{9} \zeta_{3}\right) \\
& +C_{F}^{2} n_{f}\left(\frac{102137}{243}-\frac{7024}{27} \zeta_{2}-\frac{1376}{9} \zeta_{3}\right)-C_{F} n_{f}^{2}\left(\frac{7672}{81}-\frac{128}{9} \zeta_{2}\right) \text {, } \\
& \left.x c_{\phi, \mathrm{q}}^{(3)}\right|_{L_{0}^{0}}=C_{F} C_{A}^{2}\left(\frac{19967}{36}+\frac{149944}{81} \zeta_{2}+\frac{111202}{27} \zeta_{3}-\frac{55748}{45} \zeta_{2}^{2}-\frac{608}{3} \zeta_{2} \zeta_{3}-\frac{4184}{3} \zeta_{5}\right) \\
& -C_{F}^{2} C_{A}\left(\frac{327439}{108}-\frac{82576}{27} \zeta_{2}+\frac{3088}{3} \zeta_{3}+\frac{1528}{5} \zeta_{2}^{2}+\frac{592}{3} \zeta_{2} \zeta_{3}+\frac{448}{3} \zeta_{5}\right) \\
& +C_{F}^{3}\left(\frac{6601}{6}-544 \zeta_{2}-76 \zeta_{3}-\frac{1384}{5} \zeta_{2}^{2}+64 \zeta_{2} \zeta_{3}+560 \zeta_{5}\right) \\
& +C_{F} C_{A} n_{f}\left(\frac{398771}{243}-\frac{17104}{27} \zeta_{2}-\frac{13204}{27} \zeta_{3}+\frac{896}{15} \zeta_{2}^{2}\right)+C_{F}^{2} n_{f}\left(\frac{421208}{729}\right. \\
& \left.-\frac{33896}{81} \zeta_{2}-\frac{3824}{27} \zeta_{3}+\frac{656}{15} \zeta_{2}^{2}\right)-C_{F} n_{f}^{2}\left(\frac{25291}{243}-\frac{848}{27} \zeta_{2}-\frac{16}{3} \zeta_{3}\right)
\end{aligned}
$$

and

$$
\begin{aligned}
\left.x c_{\phi, \mathrm{g}}^{(3)}\right|_{L_{0}^{5}}= & -\frac{8}{15} C_{A}^{3}, \\
\left.x c_{\phi, \mathrm{g}}^{(3)}\right|_{L_{0}^{4}}= & -\frac{220}{9} C_{A}^{3}+\frac{8}{3} C_{A}^{2} n_{f}+\frac{16}{9} C_{F} C_{A} n_{f}, \\
\left.x c_{\phi, \mathrm{g}}^{(3)}\right|_{L_{0}^{3}}= & -C_{A}^{3}\left(\frac{9698}{27}-\frac{128}{3} \zeta_{2}\right)+\frac{2032}{27} C_{A}^{2} n_{f}+\frac{296}{9} C_{F} C_{A} n_{f}-\frac{88}{27} C_{A} n_{f}^{2} \\
& -\frac{112}{27} n_{f}^{2} C_{F},
\end{aligned}
$$




$$
\begin{aligned}
& \left.x c_{\phi, \mathrm{g}}^{(3)}\right|_{L_{0}^{2}}=-C_{A}^{3}\left(\frac{61454}{27}-\frac{2200}{3} \zeta_{2}-\frac{256}{3} \zeta_{3}\right)+C_{A}^{2} n_{f}\left(\frac{16882}{27}-80 \zeta_{2}\right) \\
& +C_{F} C_{A} n_{f}\left(\frac{5872}{27}-\frac{128}{3} \zeta_{2}\right)+C_{F}^{2} n_{f}\left(\frac{44}{3}-\frac{32}{3} \zeta_{2}\right)-\frac{1184}{27} C_{A} n_{f}^{2} \\
& -\frac{760}{27} C_{F} n_{f}^{2}+\frac{8}{9} n_{f}^{3} \\
& \left.x c_{\phi, \mathrm{g}}^{(3)}\right|_{L_{0}}=-C_{A}^{3}\left(\frac{507719}{81}-\frac{95912}{27} \zeta_{2}-\frac{4136}{3} \zeta_{3}+\frac{8128}{15} \zeta_{2}^{2}\right) \\
& +C_{A}^{2} n_{f}\left(\frac{59272}{27}-\frac{19648}{27} \zeta_{2}+\frac{16}{3} \zeta_{3}\right)+C_{F}^{2} n_{f}\left(\frac{568}{9}-\frac{544}{9} \zeta_{2}+\frac{128}{3} \zeta_{3}\right) \\
& +C_{F} C_{A} n_{f}\left(\frac{181109}{243}-\frac{5296}{27} \zeta_{2}-\frac{2560}{9} \zeta_{3}\right)-C_{A} n_{f}^{2}\left(\frac{13988}{81}-\frac{256}{9} \zeta_{2}\right) \\
& -C_{F} n_{f}^{2}\left(\frac{7370}{81}-\frac{160}{9} \zeta_{2}\right)+\frac{80}{27} n_{f}^{3} \\
& \left.x c_{\phi, \mathrm{g}}^{(3)}\right|_{L_{0}^{0}}=-C_{A}^{3}\left(\frac{57560}{27}-\frac{407248}{81} \zeta_{2}-\frac{87922}{27} \zeta_{3}+\frac{9724}{5} \zeta_{2}^{2}+336 \zeta_{2} \zeta_{3}+984 \zeta_{5}\right) \\
& +C_{A}^{2} n_{f}\left(\frac{4398355}{1458}-\frac{123284}{81} \zeta_{2}-\frac{7568}{27} \zeta_{3}+\frac{5864}{45} \zeta_{2}^{2}\right) \\
& +C_{F} C_{A} n_{f}\left(\frac{881945}{1458}-\frac{8228}{27} \zeta_{2}-\frac{6752}{9} \zeta_{3}+\frac{704}{9} \zeta_{2}^{2}\right) \\
& +C_{F}^{2} n_{f}\left(\frac{3053}{54}-\frac{3112}{27} \zeta_{2}+\frac{880}{9} \zeta_{3}+\frac{832}{45} \zeta_{2}^{2}\right)-C_{A} n_{f}^{2}\left(\frac{344365}{1458}-\frac{2504}{27} \zeta_{2}\right. \\
& \left.+\frac{296}{27} \zeta_{3}\right)-C_{F} n_{f}^{2}\left(\frac{105398}{729}-\frac{1264}{27} \zeta_{2}-\frac{1552}{27} \zeta_{3}\right)+n_{f}^{3}\left(\frac{200}{81}-\frac{16}{9} \zeta_{2}\right) \text {. }
\end{aligned}
$$

This behaviour is different from that of the (spacelike) splitting functions and gauge-boson exchange coefficient functions which receive an only single-logarithmic ('BFKL') enhancement of the leading $1 / x$ contributions to all orders in $\alpha_{\mathrm{s}}$ [49-51]. In fact, this seemingly surprising feature of the $\phi G^{\mu v} G_{\mu v}$ probe of the hadronic system was already briefly stated in Ref. [50], and the leading double-logarithmic terms were explicitly calculated in Ref. [52] for the related case of Higgs production in proton-proton collisions in the heavy-top limit.

However, the functions $c_{\phi, \mathrm{p}}^{(n)}(x)$ are probed at very small values of $x$ only at very high centre-ofmass $(\mathrm{CM})$ energies $\sqrt{s}$. Given the finite physical top mass, the large top-mass approximations for Higgs exchange/production breaks down in this region. Indeed, as explicitly shown recently for Higgs production in Ref. [53], the full finite- $m_{\text {top }}$ coefficient functions receive only single logarithmic contributions at small $x$. Consequently the effective-theory coefficient functions $c_{\phi, \mathrm{p}}^{(n)}(x)$ cease to provide useful approximations for Higgs-exchange DIS in this limit. Where exactly in $x$ this occurs cannot be established without the very non-trivial calculation of at least all NNLO $1 / x$ terms in the full theory. This point has not been reached yet even in the phenomenologically important case of Higgs production in proton-(anti-)proton collisions, for the present status see Refs. [54]. 
We are now ready to discuss the numerical size of the coefficient functions (3.5) - 3.9), A.1) and (A.2). In Figs. 1 and 2 their cumulative effect is shown for $n_{f}=4$ quark flavours and an orderindependent value $\alpha_{\mathrm{s}}\left(\mu_{\mathrm{r}}^{2}=\mu_{\mathrm{f}}^{2}=Q_{0}^{2}\right)=0.2$ for the strong coupling constant corresponding to a scale of $Q_{0}^{2} \approx 20 \ldots 50 \mathrm{GeV}^{2}$. The same reference point was used in Refs. [8,9], hence the present results can be compared directly to the photon-exchange case discussed in those articles. Note that Fig. 2 does not provide the full information about the effect of the gluon coefficient function $C_{\phi, \mathrm{g}}(x)$ due to presence of +-distributions, recall Eq. (3.11), and $\delta(1-x)$ contributions.

As shown in the right parts of both figures, the perturbative expansions of $C_{\phi, \mathrm{p}}(x)$ are rather stable in the mid- $x$ region, but, unsurprisingly, large beyond-NLO corrections are found in the soft-gluon large- $x$ and the high-energy small- $x$ regions. While in the former the NNLO and $N^{3} L O$ are similar, the perturbative expansion appears to break down below $x \approx 10^{-3}$ and $x \approx 10^{-4}$ for $C_{\phi, \mathrm{q}}(x)$ and $C_{\phi, \mathrm{g}}(x)$, respectively. This breakdown thus occurs in a region where this coefficient functions are definitely not expected to represent Higgs exchange anymore, as a CM energy of about $m_{\text {top }}$ is required to access $x=10^{-3}$ at the chosen scale $Q^{2} \approx 30 \mathrm{GeV}^{2}$.

Obviously the (perturbative) coefficient functions enter physical quantities such as the structure function $F_{\phi}$ only through convolutions such as

$$
\left[C_{\phi, \mathrm{q}} \otimes q\right](x)=\int_{x}^{1} \frac{d y}{y} C_{\phi, \mathrm{q}}(x) q\left(\frac{x}{y}\right)
$$

with the (partly non-perturbative) quark and gluon distributions which, due to the general shape of $q_{\mathrm{S}}(x)$ and $g(x)$, shift the onset of the small- $x$ instability to considerably lower values of $x$ than in Figs. 1 and 2. The resulting contributions of Eqs. (3.5) - (3.9), (A.1) and (A.2) to $F_{\phi}$ are illustrated in Fig. 3 for the schematic, but sufficiently realistic order-independent input

$$
\begin{aligned}
& x q_{\mathrm{s}}\left(x, Q_{0}^{2}\right)=0.6 x^{-0.3}(1-x)^{3.5}\left(1+5.0 x^{0.8}\right), \\
& x g\left(x, Q_{0}^{2}\right)=1.6 x^{-0.3}(1-x)^{4.5}\left(1-0.6 x^{0.3}\right)
\end{aligned}
$$

already employed in Refs. [7-9]. In reality both $\alpha_{\mathrm{s}}$ and the parton distributions do depend on the perturbative order in a manner that reduces the relative higher-order corrections in particular at large $x$. Keeping this in mind, the medium- $Q^{2}$ perturbative stability in Fig. 3 is satisfactory over a wide range in $x$ despite, as expected for a gluon-dominated quantity, the presence of considerably larger $\alpha_{\mathrm{s}}$ corrections than found for the standard-DIS structure function $F_{2}$.

Finally we need to address the Mellin moments of the new coefficient functions $c_{\phi, \mathrm{p}}^{(n)}(x)$ for $n=1,2,3(\mathrm{p}=\mathrm{q}, \mathrm{g})$. The second moments for the quark case are given by

$$
\begin{aligned}
c_{\phi, \mathrm{q}}^{(1)}(N=2) & =-\frac{4}{3} C_{F} \cong-1.77778, \\
c_{\phi, \mathrm{q}}^{(2)}(N=2) & =-C_{F} C_{A}\left(\frac{6224}{81}-32 \zeta_{3}\right)+C_{F}^{2}\left(\frac{1237}{81}-32 \zeta_{3}\right)+\frac{44}{3} C_{F} n_{f} \\
& \cong-194.729+19.5556 n_{f},
\end{aligned}
$$



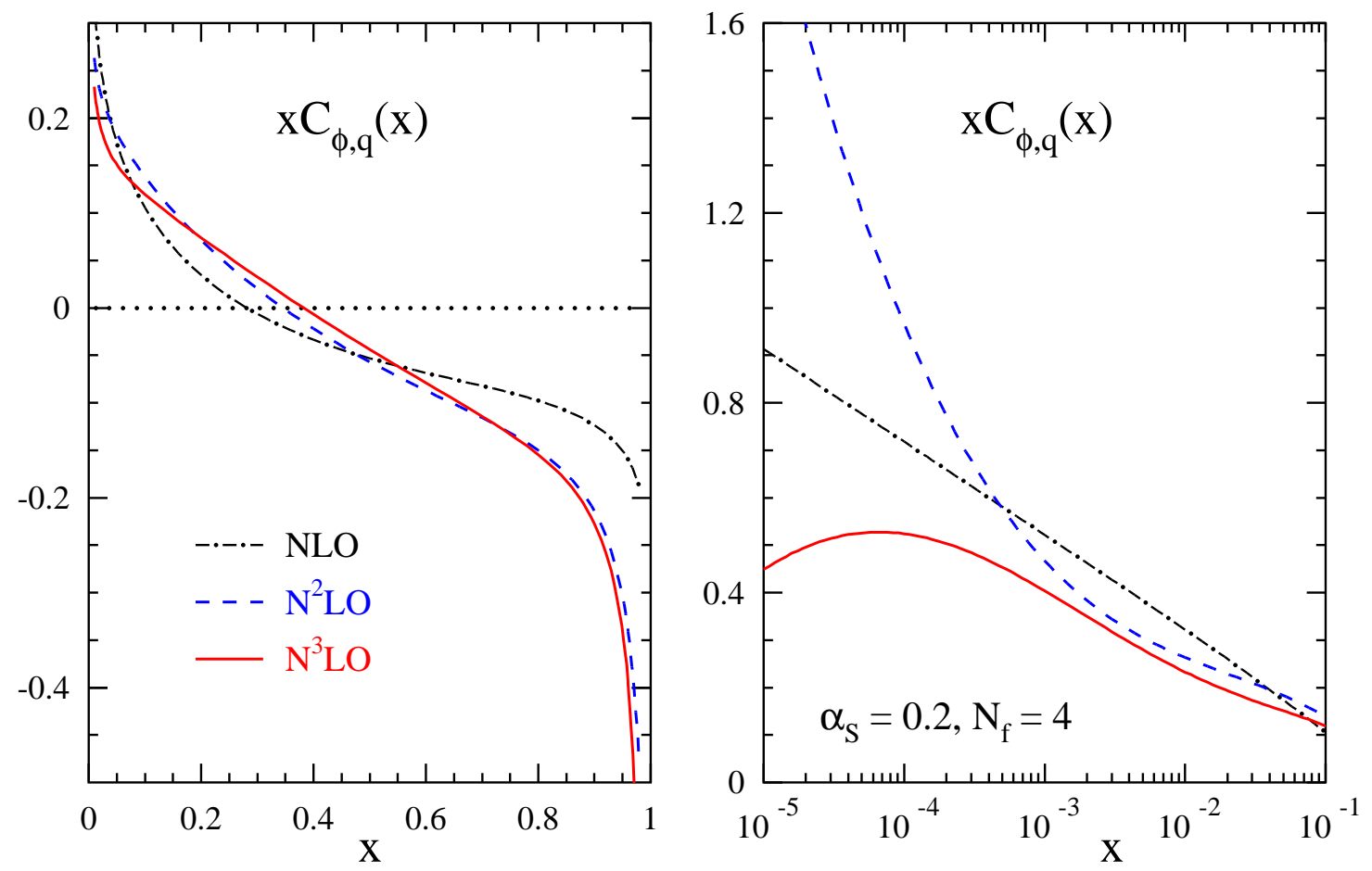

Figure 1: The perturbative expansion to order $\alpha_{\mathrm{s}}^{3}\left(\mathrm{~N}^{3} \mathrm{LO}\right)$ of the quark coefficient function $C_{\phi, \mathrm{q}}(x)$ for DIS via the exchange of a scalar $\phi$ with a $\phi G^{\mu v} G_{\mu v}$ coupling to gluons. The results are shown for a standard medium-scale reference point and have been multiplied by $x$ for display purposes.
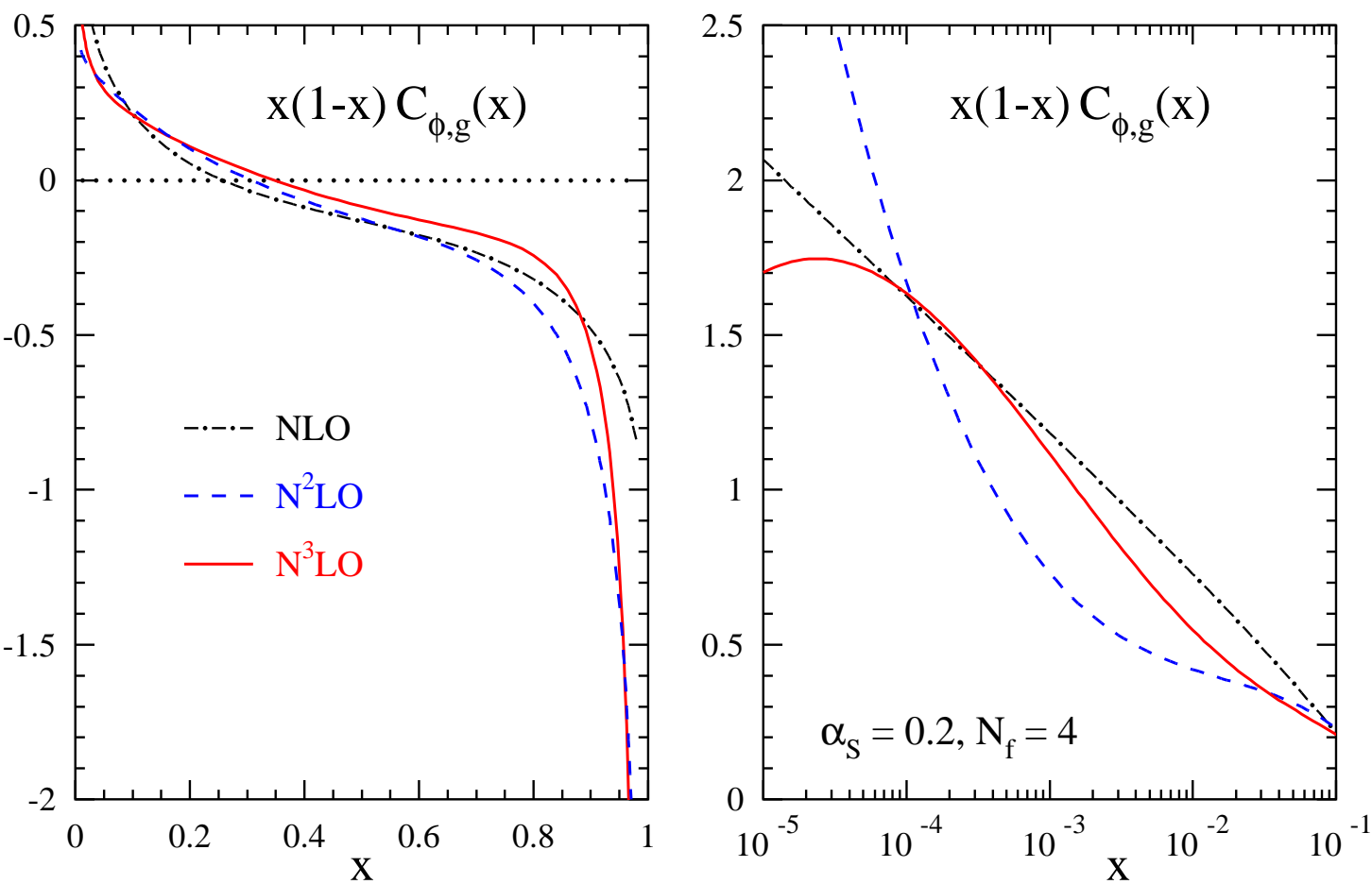

Figure 2: As Fig. 1, but for the gluon coefficient function $C_{\phi, \mathrm{g}}(x)$ at $x<1$, multiplied by an additional factor $(1-x)$ compensating the large $x$-dependence due to the + -distribution contributions. 

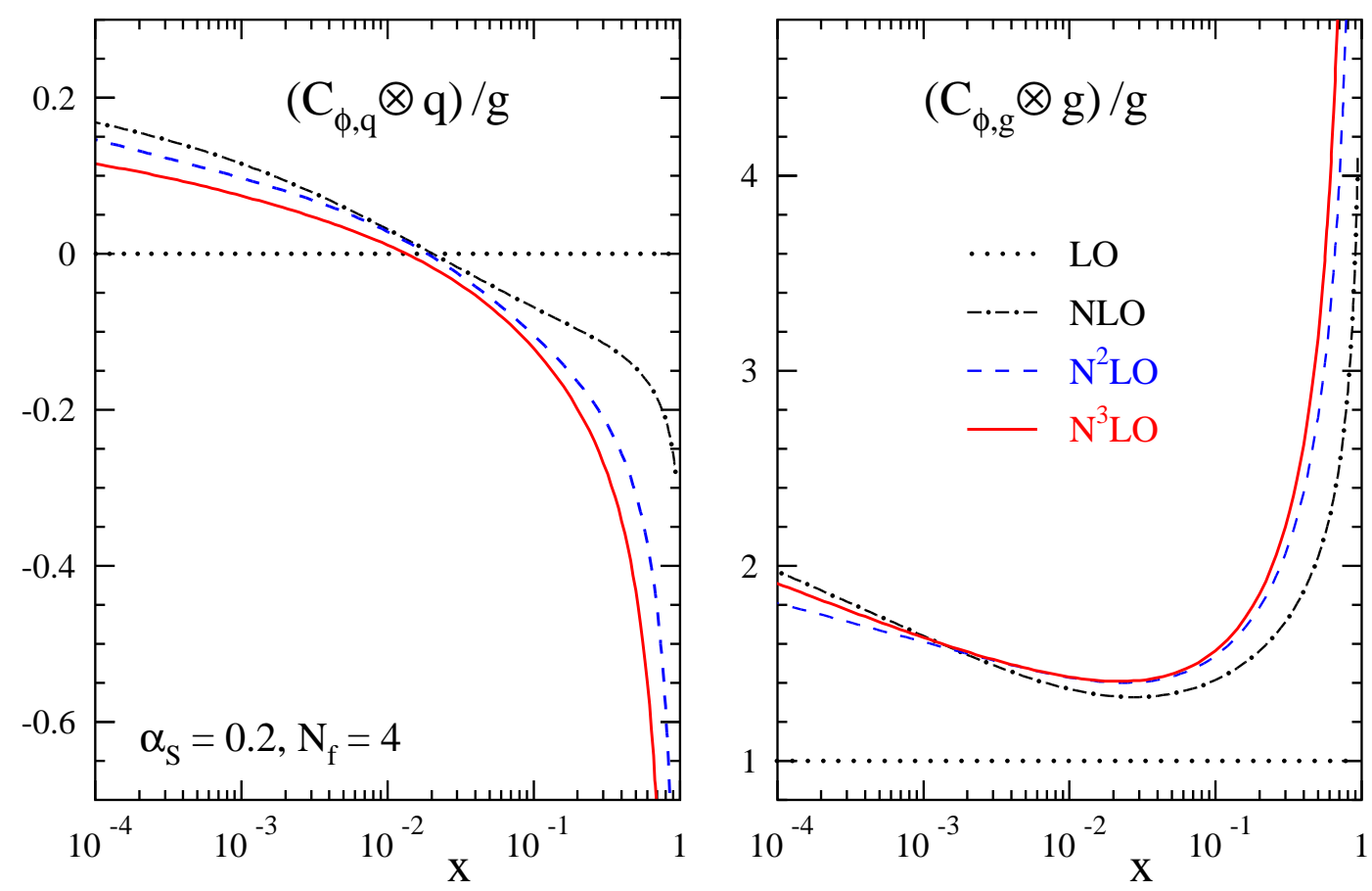

Figure 3: The perturbative expansion of the quark (left) and gluon (right) contributions to the structure function $F_{\phi}$, normalized to the lowest-order result $F_{\phi}\left(x, Q^{2}\right)=x g\left(x, Q^{2}\right)$, for the orderindependent input (3.51) at our reference point corresponding to a scale $Q^{2} \approx 20 \ldots 50 \mathrm{GeV}^{2}$.

$$
\begin{aligned}
c_{\phi, \mathrm{q}}^{(3)}(N=2)= & -C_{F} C_{A}^{2}\left(\frac{10744957}{4374}-\frac{121232}{81} \zeta_{3}+\frac{64}{15} \zeta_{2}^{2}+\frac{640}{3} \zeta_{5}\right) \\
& +C_{F}^{2} C_{A}\left(\frac{4294603}{4374}-\frac{6488}{9} \zeta_{3}+\frac{64}{5} \zeta_{2}^{2}-\frac{1280}{3} \zeta_{5}\right) \\
& -C_{F}^{3}\left(\frac{137462}{729}+\frac{41792}{81} \zeta_{3}+\frac{128}{15} \zeta_{2}^{2}-\frac{2560}{3} \zeta_{5}\right) \\
& -C_{F}^{2} n_{f}\left(\frac{62201}{2187}-\frac{11264}{81} \zeta_{3}+\frac{128}{15} \zeta_{2}^{2}\right)-C_{F} n_{f}^{2}\left(\frac{33854}{729}-\frac{64}{9} \zeta_{3}\right) \\
& +C_{F} C_{A} n_{f}\left(\frac{1616063}{2187}-\frac{22768}{81} \zeta_{3}+\frac{128}{15} \zeta_{2}^{2}\right) \\
\cong & -12116.73+1902.16 n_{f}-50.5213 n_{f}^{2}
\end{aligned}
$$

where the second (approximate) equalities are obtained by inserting $C_{A}=3, C_{F}=4 / 3$ and the numerical values of the $\zeta$-function. The results for the gluon coefficient function read

$$
\begin{aligned}
c_{\phi, \mathrm{g}}^{(1)}(N=2)= & \frac{203}{18} C_{A}-\frac{10}{9} n_{f} \cong 33.8333-1.11111 n_{f}, \\
c_{\phi, \mathrm{g}}^{(2)}(N=2)= & C_{A}^{2}\left(\frac{23473}{108}-66 \zeta_{3}\right)-C_{A} n_{f}\left(\frac{8215}{162}+12 \zeta_{3}\right) \\
& -C_{F} n_{f}\left(\frac{1726}{81}-24 \zeta_{3}\right)+\frac{100}{81} n_{f}^{2} \\
\cong & 1242.06-185.349 n_{f}+1.23457 n_{f}^{2},
\end{aligned}
$$




$$
\begin{aligned}
c_{\phi, \mathrm{g}}^{(3)}(N=2)= & C_{A}^{3}\left(\frac{27878711}{5832}-\frac{66970}{27} \zeta_{3}+\frac{880}{3} \zeta_{5}\right) \\
& -C_{F} C_{A} n_{f}\left(\frac{2824243}{2916}-652 \zeta_{3}-\frac{104}{15} \zeta_{2}^{2}-\frac{760}{3} \zeta_{5}\right) \\
& -C_{A}^{2} n_{f}\left(\frac{3723227}{2187}-\frac{4162}{81} \zeta_{3}+\frac{24}{5} \zeta_{2}^{2}-\frac{280}{3} \zeta_{5}\right) \\
& +C_{F}^{2} n_{f}\left(\frac{40081}{486}+\frac{24668}{81} \zeta_{3}-\frac{32}{15} \zeta_{2}^{2}-\frac{1360}{3} \zeta_{5}\right)-\frac{1000}{729} n_{f}^{3} \\
& +C_{F} n_{f}^{2}\left(\frac{53945}{486}-\frac{8360}{81} \zeta_{3}\right)+C_{A} n_{f}^{2}\left(\frac{1155203}{8748}+\frac{3652}{81} \zeta_{3}\right) \\
\cong & 56778.8-13673.8 n_{f}+541.328 n_{f}^{2}-1.37174 n_{f}^{3} .
\end{aligned}
$$

Note the conspicuous absence of coefficient linear in $\zeta_{2}$ from the above second- and third-order results, a feature that has been observed and discussed before for the corresponding moments of the gauge-boson exchange structure functions in Refs. [23].

For $n_{f}=4$ flavours Eqs. (3.52) - 3.57) yield the rather benign expansions

$$
\begin{aligned}
& C_{\phi, \mathrm{q}}(N=2) \cong-0.1415 \alpha_{\mathrm{s}}-0.7378 \alpha_{\mathrm{s}}^{2}-2.6791 \alpha_{\mathrm{s}}^{3}+\ldots \\
& C_{\phi, \mathrm{g}}(N=2) \cong 1+2.3387 \alpha_{\mathrm{s}}+3.2956 \alpha_{\mathrm{s}}^{2}+5.3704 \alpha_{\mathrm{s}}^{3}+\ldots
\end{aligned}
$$

which nevertheless, as expected, are rather different from their counterparts for the photon-exchange structure function $F_{2}$ given by [23]

$$
\begin{aligned}
& C_{2, \mathrm{q}}(N=2) \cong 1+0.0354 \alpha_{\mathrm{s}}-0.0785 \alpha_{\mathrm{s}}^{2}-0.1986 \alpha_{\mathrm{s}}^{3}+\ldots \\
& C_{2, \mathrm{~g}}(N=2) \cong \quad-0.1592 \alpha_{\mathrm{s}}-0.2259 \alpha_{\mathrm{s}}^{2}-0.0274 \alpha_{\mathrm{s}}^{3}+\ldots
\end{aligned}
$$

Consequently, as already indicated at the end of Section 2, the scheme-transformation relation (2.26) is not fulfilled with the choice (2.27) involving Eqs. (3.58) - (3.61).

The $N$-dependences of $C_{\phi, \mathrm{q}}$ and $C_{\phi, \mathrm{g}}$ are shown graphically, again for $\alpha_{\mathrm{s}}=0.2$, in Fig. 4 . The corresponding results for $F_{2}$ and $F_{L}$ in standard DIS have been presented in Figs. 11 and 12 of Ref. [9]. The present pattern is rather similar to that for $F_{2}$ (with the quark and gluon coefficient functions interchanged), albeit with considerably larger $\alpha_{\mathrm{s}}$ corrections. In fact, the $N$-dependent values of the strong coupling,

$$
\widehat{\alpha}_{\phi, i}^{(n)}(N)=4 \pi \frac{c_{\phi, i}^{(n-1)}(N)}{2 c_{\phi, i}^{(n)}(N)},
$$

for which the $n$-th order corrections are half as large as those of the previous order - recall that the coefficient functions are expressed in terms of the small expansion parameter $a_{\mathrm{s}} \equiv \alpha_{\mathrm{s}} /(4 \pi)$ of Eq. (2.10) - shown in Fig. 5 are rather more similar to those for $F_{L}$ than for $F_{2}$ which can be found, respectively, in Figs. 13 and 14 of Ref. [9]. Note, however, that also in Fig. 5 the values for $\widehat{\alpha}^{(n)}(N)$ increase, rather than decrease, with the perturbative order $n$. This behaviour indicates that also here we have not yet reached the expected asymptotic regime of the expansion in powers of $\alpha_{\mathrm{S}}$ with a factorial-type growth $c^{(n)} \sim n$ ! of the expansion coefficients. 

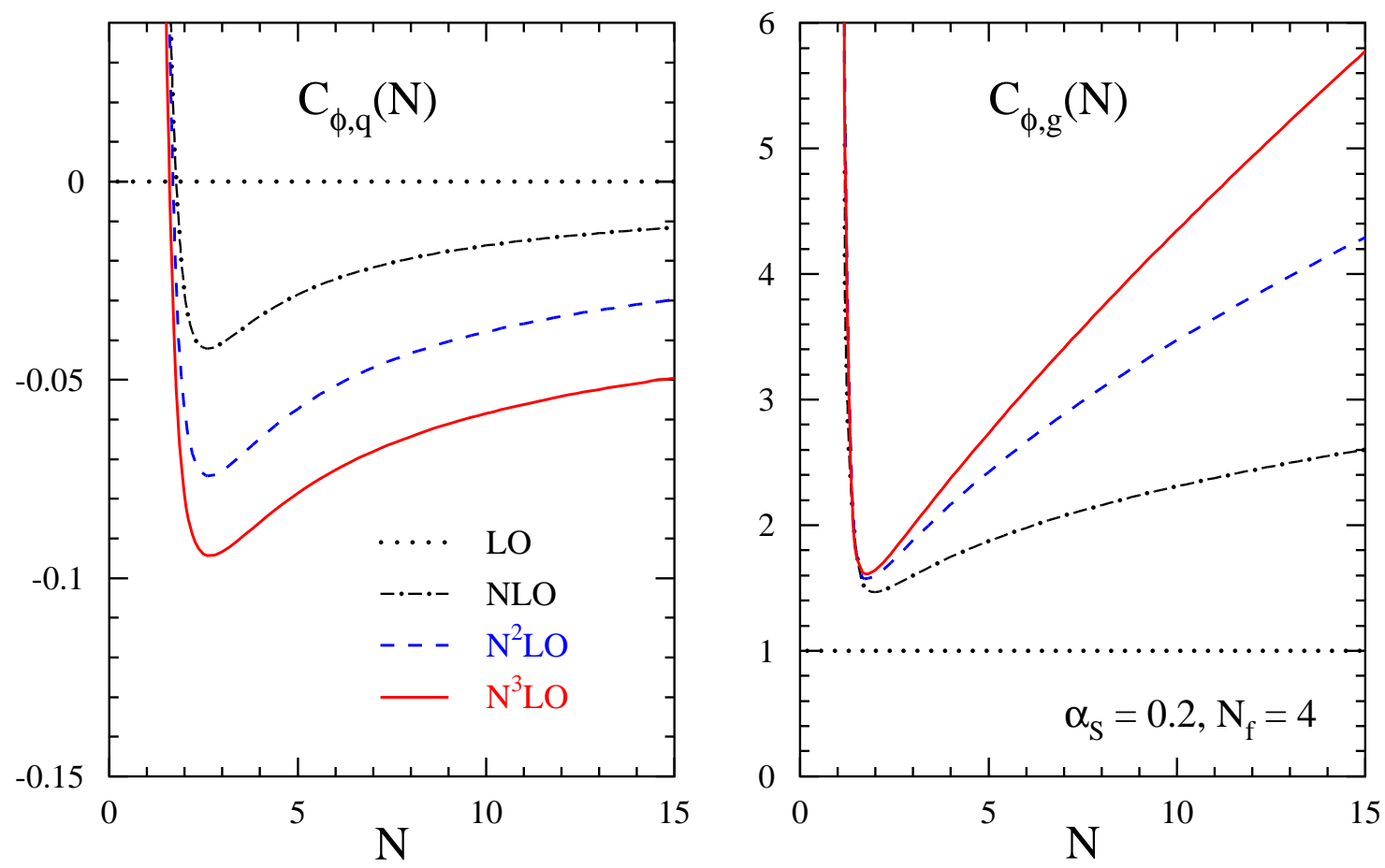

Figure 4: The perturbative expansion to order $\alpha_{\mathrm{s}}^{3}\left(\mathrm{~N}^{3} \mathrm{LO}\right)$ of the quark and gluon coefficient functions for the structure function $F_{\phi}$ in Mellin- $N$ space, shown for four effectively massless flavours and the order-independent value of $\alpha_{\mathrm{s}}$ specified in the right panel.
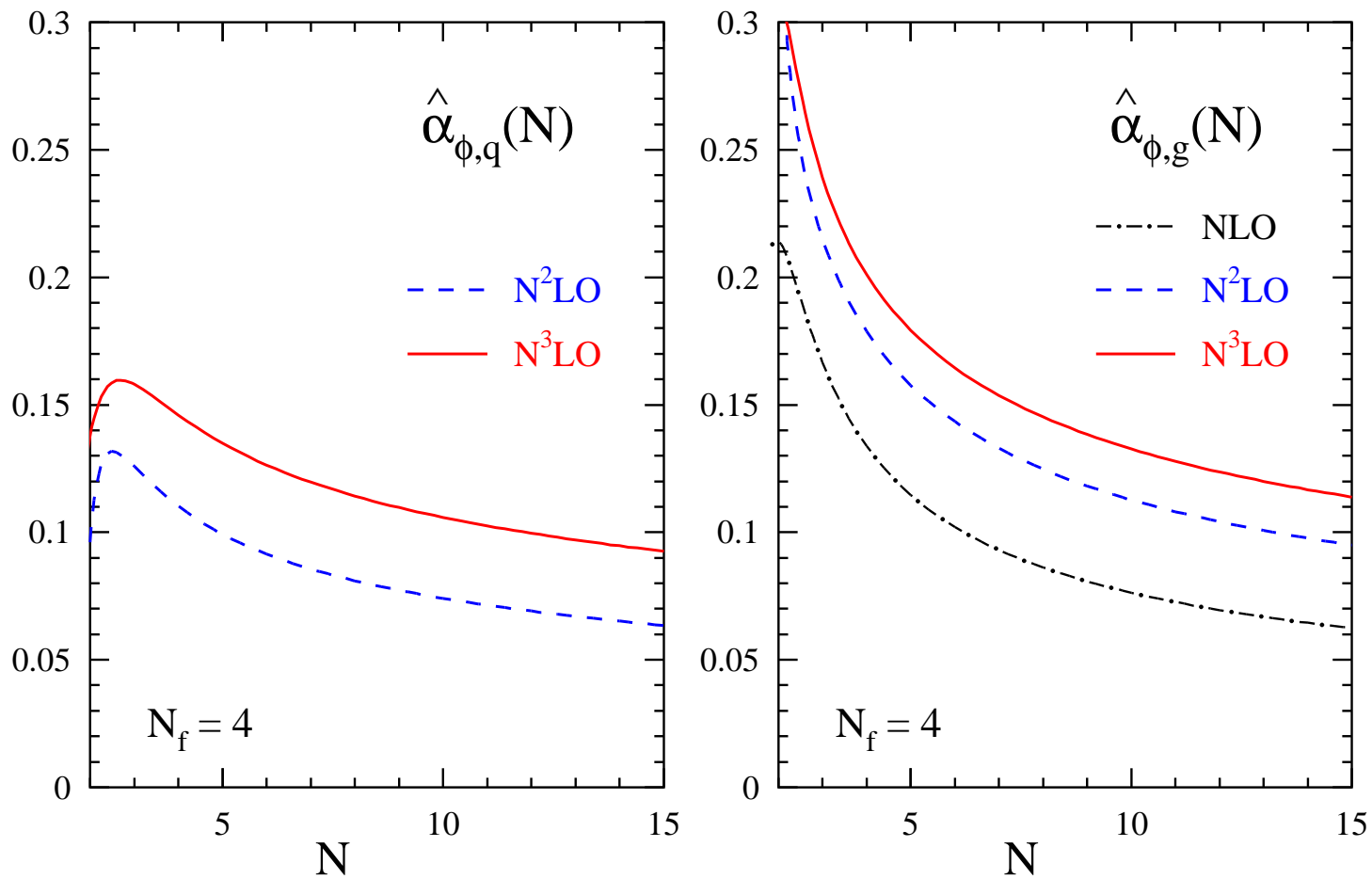

Figure 5: The $N$-dependent values (3.62) of $\alpha_{\mathrm{s}}$ at which the effect of the $n$-th order $\left(\mathrm{N}^{n} \mathrm{LO}\right)$ quark and gluon coefficient functions for $F_{\phi}$ is half as large as that of the previous order. A NLO curve can only be shown for the gluon case (right) since only here the LO contribution does not vanish. 


\section{Physical evolution kernel for $F_{2}$ and $F_{\phi}$}

We are now in a position to write down and discuss the physical kernels for the coupled flavoursinglet evolution of the structure functions $F_{2}$ and $F_{\phi}$ as defined at the end of Section 2. To NNLO $(l=2)$ these kernels,

$$
K_{a b}=\sum_{l=0} a_{\mathrm{s}}^{l+1} K_{a b}^{(l)} \quad \text { with } \quad a, b=2, \phi,
$$

are specified in terms of the three-loop splitting functions $[6,7]$ together with the second-order coefficient functions for $F_{2}$, first calculated in Refs. [55], and $F_{\phi}$ provided in the previous section. Also these results will by given in terms of harmonic polylogarithms [45] as summarized in Eqs. (3.1) - (3.4) above. From $l=1$ we will present $K_{22}$ in the form

$$
K_{22}^{(l)}=K_{2, \mathrm{~ns}}^{(l)}+K_{22, \mathrm{ps}}^{(l)},
$$

i.e., we separately provide the scalar evolution kernel for the non-singlet structure function $F_{2, \text { ns }}$ (addressed, but not written down in this form, before in Refs. [20,56]), which is of direct phenomenological interest for, e.g., the determination of $\alpha_{\mathrm{s}}$ from scaling violations. As above, our results are given in the $\overline{\mathrm{MS}}$ renormalization scheme for the scale $\mu=Q$.

At leading order (LO) the elements of the physical kernel are identical to the splitting functions, recall the first line of Eq. (2.24), i.e.,

$$
\begin{aligned}
K_{22}^{(0)} & =C_{F}\left(2 p_{\mathrm{qq}}(x)+3 \delta(1-x)\right), \\
K_{2 \phi}^{(0)} & =2 n_{f} p_{\mathrm{qg}}(x), \\
K_{\phi 2}^{(0)} & =2 C_{F} p_{\mathrm{gq}}(x), \\
K_{\phi \phi}^{(0)} & =C_{A}\left(4 p_{\mathrm{gg}}(x)+\frac{11}{3} \delta(1-x)\right)-\frac{2}{3} n_{f} \delta(1-x),
\end{aligned}
$$

where we have used Eq. (3.7) and the additional abbreviations

$$
\begin{aligned}
& p_{\mathrm{qq}}(x)=2(1-x)^{-1}-1-x \\
& p_{\mathrm{qg}}(x)=1-2 x+2 x^{2} .
\end{aligned}
$$

The next-to-leading order (NLO) contributions to these kernels are given by

$$
\begin{aligned}
& K_{2, \mathrm{~ns}}^{(1)}(x)=C_{F}^{2}\left(p_{\mathrm{qq}}(x)\left[-6 \mathrm{H}_{0}+8 \mathrm{H}_{1,0}+8 \mathrm{H}_{2}\right]+8 p_{\mathrm{qq}}(-x)\left[-\zeta_{2}-2 \mathrm{H}_{-1,0}+\mathrm{H}_{0,0}\right]\right. \\
& \left.\quad+2\left(-2+2 x+\mathrm{H}_{0}-3 x \mathrm{H}_{0}-2 \mathrm{H}_{0,0}-2 x \mathrm{H}_{0,0}\right)+\left[3 / 2-12 \zeta_{2}+24 \zeta_{3}\right] \delta(1-x)\right) \\
& +C_{A} C_{F}\left(p_{\mathrm{qq}}(x)\left[367 / 18+2 / 3\left(-6 \zeta_{2}+22 \mathrm{H}_{0}+6 \mathrm{H}_{0,0}+11 \mathrm{H}_{1}\right)\right]\right. \\
& \left.\quad+4 p_{\mathrm{qq}}(-x)\left[\zeta_{2}+2 \mathrm{H}_{-1,0}-\mathrm{H}_{0,0}\right]+1 / 6\left(13-167 x+\left[215+176 \zeta_{2}-72 \zeta_{3}\right] \delta(1-x)\right)\right) \\
& +C_{F} n_{f}\left(p_{\mathrm{qq}}(x)\left[-29 / 9-8 / 3 \mathrm{H}_{0}-4 / 3 \mathrm{H}_{1}\right]+1 / 3\left(1+13 x-\left[19+16 \zeta_{2}\right] \delta(1-x)\right)\right),
\end{aligned}
$$




$$
\begin{aligned}
& K_{22, \mathrm{ps}}^{(1)}(x)=C_{F} n_{f}\left(2 / 3\left(32 x^{-1}-69+45 x-8 x^{2}\right)+4 / 3\left(4 x^{-1}-9 x+8 x^{2}\right) \mathrm{H}_{0}\right. \\
& \left.-8(1+x) \mathrm{H}_{0,0}\right) \text {, } \\
& K_{2 \phi}^{(1)}(x)=C_{A} n_{f}\left(8 p_{\mathrm{qg}}(x) \mathrm{H}_{2}-8 p_{\mathrm{qg}}(-x) \mathrm{H}_{-1,0}+2 / 3\left(1-50 x+50 x^{2}\right) \mathrm{H}_{1}\right. \\
& -8(1+2 x) \mathrm{H}_{0,0}-8 \zeta_{2}\left(1+2 x^{2}\right)+2 / 3\left(8 x^{-1}-11-98 x+66 x^{2}\right) \mathrm{H}_{0} \\
& \left.+2 / 9\left(100 x^{-1}-322+155 x+18 x^{2}\right)\right)+C_{F} n_{f}\left(4 p_{\mathrm{qg}}(x)\left[2 \mathrm{H}_{0,0}-3 \mathrm{H}_{1}+2 \mathrm{H}_{1,0}\right]\right. \\
& \left.-4(1-2 x) \mathrm{H}_{0,0}+2\left(1+4 x-12 x^{2}\right) \mathrm{H}_{0}+2\left(2-7 x+4 x^{2}\right)+16 \mathrm{H}_{1}\right) \\
& +n_{f}^{2}\left(4 / 3 p_{\mathrm{qg}}(x)\left[\mathrm{H}_{0}+\mathrm{H}_{1}\right]+4 / 9\left(-2 x^{-1}+11-25 x+21 x^{2}\right)\right) \text {, } \\
& K_{\phi 2}^{(1)}(x)=C_{F}^{2}\left(8 p_{\mathrm{gq}}(x) \mathrm{H}_{2}+8 \zeta_{2}\left(-2 x^{-1}+2-x\right)-4(2-x) \mathrm{H}_{0,0}+2(2+3 x) \mathrm{H}_{0}\right. \\
& \left.+\left(10 x^{-1}-18-x\right)+12 x^{-1} \mathrm{H}_{1}\right)+C_{A} C_{F}\left(p_{\mathrm{gq}}(x)\left[4 / 3 \mathrm{H}_{1}+8 \mathrm{H}_{1,0}\right]\right. \\
& -8 p_{\mathrm{gq}}(-x) \mathrm{H}_{-1,0}-6(2-x) \mathrm{H}_{1}+8(2+x) \mathrm{H}_{0,0}+1 / 3\left(87 x^{-1}-22+83 x+64 x^{2}\right) \\
& \left.+2 / 3\left(22 x^{-1}-138+15 x-16 x^{2}\right) \mathrm{H}_{0}+8 \zeta_{2}\left(2 x^{-1}+x\right)\right) \\
& +C_{F} n_{f}\left(p_{\mathrm{gq}}(x)\left[-4 \mathrm{H}_{0}-4 / 3 \mathrm{H}_{1}\right]-2 / 3\left(29 x^{-1}-26+13 x\right)\right) \text {, } \\
& K_{\phi \phi}^{(1)}(x)=C_{A}^{2}\left(p_{\mathrm{gg}}(x)\left[389 / 9-8 \zeta_{2}+44 / 3 \mathrm{H}_{0}+8 \mathrm{H}_{0,0}+44 / 3 \mathrm{H}_{1}+16 \mathrm{H}_{1,0}+16 \mathrm{H}_{2}\right]\right. \\
& -8 p_{\mathrm{gg}}(-x)\left[\zeta_{2}+2 \mathrm{H}_{-1,0}-\mathrm{H}_{0,0}\right]+32(1+x) \mathrm{H}_{0,0}-4 / 3\left(25-11 x+44 x^{2}\right) \mathrm{H}_{0} \\
& \left.-1 / 9\left(268 x^{-1}-728+607 x-389 x^{2}\right)-\left(449 / 27-44 / 3 \zeta_{2}-12 \zeta_{3}\right) \delta(1-x)\right) \\
& +C_{A} n_{f}\left(4 / 3 p_{\mathrm{gg}}(x)\left[-7-2 \mathrm{H}_{0}-2 \mathrm{H}_{1}\right]-8 / 3(1+x) \mathrm{H}_{0}-4 / 9\left(13 x^{-1}+13-2 x-2 x^{2}\right)\right. \\
& \left.+4 / 27\left(43-18 \zeta_{2}\right) \delta(1-x)\right)+C_{F} n_{f}\left(-8(1+x) \mathrm{H}_{0,0}-4 / 3\left(4 x^{-1}+6-9 x\right) \mathrm{H}_{0}\right. \\
& \left.-2 / 9\left(44 x^{-1}-27-45 x+28 x^{2}\right)-2 \delta(1-x)\right) \\
& +n_{f}^{2}\left(4 / 9 p_{\operatorname{gg}}(x)+4 / 9\left(2-x+x^{2}\right)-20 / 27 \delta(1-x)\right) \text {. }
\end{aligned}
$$

The five corresponding third-order (NNLO) corrections fill about 10 pages (in total) and therefore are deferred to Appendix B.

The leading large- $x$ contributions to the evolution kernels $(4.8)-(4.12)$ and $(\overline{B .1})-(B .5)$ are given by terms of the form $\mathcal{D}_{k}$ (defined in Eq. (3.10) ) for the diagonal entries $K_{22}^{(n)}$ and $K_{\phi \phi}^{(n)}$, and $L_{1}^{k} \equiv \ln ^{k}(1-x)$ for their off-diagonal counterparts $K_{2 \phi}^{(n)}$ and $K_{\phi 2}^{(n)}$. It turns out that in all cases the higher-order enhancement (as far as known, obviously) is only single-logarithmic, $k=0, \ldots, n$. This is in contrast to the behaviour of the off-diagonal $\overline{\mathrm{MS}}$ splitting functions, cf. Eqs. (4.17) (4.19) of Ref. [7], and of all coefficient functions for $F_{2}$ and $F_{\phi}$ as discussed in Ref. [9] and Section 3 and Appendix $C$ of the present article. Hence the key non-singlet observation of Refs. $[19,20]$ generalizes to the present flavour-singlet case. We will utilize this fact in the next section. 
The NLO and NNLO large- $x$ coefficients for $K_{22}(x)$ are given by

$$
\begin{aligned}
& \left.K_{22}^{(1)}\right|_{\mathcal{D}_{1}}=-\frac{44}{3} C_{F} C_{A}+\frac{8}{3} C_{F} n_{f} \\
& \left.K_{22}^{(1)}\right|_{\mathcal{D}_{0}}=\left(\frac{367}{9}-8 \zeta_{2}\right) C_{F} C_{A}-\frac{58}{9} C_{F} n_{f}
\end{aligned}
$$

and

$$
\begin{aligned}
\left.K_{22}^{(2)}\right|_{\mathcal{D}_{2}}= & \frac{484}{9} C_{F} C_{A}^{2}-\frac{176}{9} C_{F} C_{A} n_{f}+\frac{16}{9} C_{F} n_{f}^{2} \\
\left.K_{22}^{(2)}\right|_{\mathcal{D}_{1}}= & -\left(\frac{9298}{27}-\frac{176}{3} \zeta_{2}\right) C_{F} C_{A}^{2}+\left(\frac{3104}{27}-\frac{32}{3} \zeta_{2}\right) C_{F} C_{A} n_{f} \\
& +8 C_{F}^{2} n_{f}-\frac{232}{27} C_{F} n_{f}^{2}, \\
\left.K_{22}^{(2)}\right|_{\mathcal{D}_{0}=}= & \left(\frac{50689}{81}-\frac{680}{3} \zeta_{2}-264 \zeta_{3}+\frac{176}{5} \zeta_{2}^{2}\right) C_{F} C_{A}^{2} \\
& +\left(11-88 \zeta_{2}+176 \zeta_{3}\right) C_{F}^{2} C_{A}-\left(\frac{15062}{81}-16 \zeta_{3}-\frac{512}{9} \zeta_{2}\right) C_{F} C_{A} n_{f} \\
& -\left(\frac{134}{3}-16 \zeta_{2}\right) C_{F}^{2} n_{f}+\left(\frac{940}{81}-\frac{288}{81} \zeta_{2}\right) C_{F} n_{f}^{2} .
\end{aligned}
$$

The corresponding terms for $K_{\phi \phi}^{(1)}(x)$ and $K_{\phi \phi}^{(2)}(x)$ read

$$
\begin{aligned}
\left.K_{\phi \phi}^{(1)}\right|_{\mathcal{D}_{1}} & =-\frac{44}{3} C_{A}^{2}+\frac{8}{3} C_{A} n_{f} \\
\left.K_{\phi \phi}^{(1)}\right|_{\mathcal{D}_{0}} & =\left(\frac{389}{9}-8 \zeta_{2}\right) C_{A}^{2}-\frac{28}{3} C_{A} n_{f}+\frac{4}{9} n_{f}^{2}
\end{aligned}
$$

and

$$
\begin{aligned}
\left.K_{\phi \phi}^{(2)}\right|_{\mathcal{D}_{2}}= & \frac{484}{9} C_{A}^{3}-\frac{176}{9} C_{A}^{2} n_{f}+\frac{16}{9} C_{A} n_{f}^{2} \\
\left.K_{\phi \phi}^{(2)}\right|_{\mathcal{D}_{1}}= & -\left(\frac{9782}{27}-\frac{176}{3} \zeta_{2}\right) C_{A}^{3}+\left(\frac{3764}{27}-\frac{32}{3} \zeta_{2}\right) C_{A}^{2} n_{f} \\
& +8 C_{F} C_{A} n_{f}-\frac{424}{27} C_{A} n_{f}^{2}+\frac{16}{27} n_{f}^{3}, \\
\left.K_{\phi \phi}^{(2)}\right|_{\mathcal{D}_{0}=}= & \left(18974 / 27-\frac{3008}{9} \zeta_{2}-88 \zeta_{3}+\frac{176}{5} \zeta_{2}^{2}\right) C_{A}^{3} \\
& -\left(20858 / 81-96 \zeta_{2}+16 \zeta_{3}\right) C_{A}^{2} n_{f}-\left(\frac{176}{3}-32 \zeta_{3}\right) C_{F} C_{A} n_{f} \\
& +\left(\frac{2284}{81}-\frac{64}{9} \zeta_{2}\right) C_{A} n_{f}^{2}+4 C_{F} n_{f}^{2}-\frac{80}{81} n_{f}^{3} .
\end{aligned}
$$


Note that the leading large- $x$ coefficients at all three orders are simply given by $\left(-\beta_{0}\right)^{n} A_{1, \mathrm{p}}$, where $A_{1, \mathrm{p}}$ denotes the appropriate one-loop quark or gluon cusp anomalous dimension, i.e., the coefficient of $(1-x)^{-1}$ in Eq. (4.3) or (4.6).

The log-enhanced large- $x$ contributions to $K_{2 \phi}^{(1)}(x)$ and $K_{2 \phi}^{(2)}(x)$ are

$$
\left.K_{2 \phi}^{(1)}\right|_{L_{1}}=-\frac{2}{3} C_{A} n_{f}-4 C_{F} n_{f}-\frac{4}{3} n_{f}^{2}
$$

and

$$
\begin{aligned}
\left.K_{2 \phi}^{(2)}\right|_{L_{1}^{2}}= & -\left(\frac{118}{3}-16 \zeta_{2}\right) C_{F} C_{A} n_{f}+\left(\frac{100}{3}-16 \zeta_{2}\right) C_{A}^{2} n_{f} \\
& +20 C_{F}^{2} n_{f}+\frac{8}{3} C_{A} n_{f}^{2}+\frac{4}{3} C_{F} n_{f}^{2} \\
\left.K_{2 \phi}^{(2)}\right|_{L_{1}}= & -\left(2-64 \zeta_{2}+96 \zeta_{3}\right) C_{F}^{2} n_{f}-\left(\frac{110}{3}+\frac{64}{3} \zeta_{2}-96 \zeta_{3}\right) C_{F} C_{A} n_{f} \\
& -24 C_{A}^{2} n_{f}+\left(\frac{8}{3}+\frac{16}{3} \zeta_{2}\right) C_{F} n_{f}^{2} .
\end{aligned}
$$

The corresponding terms for $K_{\phi 2}(x)$ are given by

$$
\left.K_{\phi 2}^{(1)}\right|_{L_{1}}=\frac{14}{3} C_{F} C_{A}-12 C_{F}^{2}+\frac{4}{3} C_{F} n_{f}
$$

and

$$
\begin{aligned}
\left.K_{\phi 2}^{(2)}\right|_{L_{1}^{2}}= & \left(16+32 \zeta_{2}\right) C_{F}^{3}+\left(34-64 \zeta_{2}\right) C_{F}^{2} C_{A}-\left(\frac{208}{9}-32 \zeta_{2}\right) C_{F} C_{A}^{2} \\
& -12 C_{F}^{2} n_{f}+\frac{20}{9} C_{F} C_{A} n_{f}+\frac{8}{9} C_{F} n_{f}^{2} \\
\left.K_{\phi 2}^{(2)}\right|_{L_{1}}= & \left(106+24 \zeta_{2}-48 \zeta_{3}\right) C_{F}^{3}-\left(\frac{2024}{3}+\frac{212}{3} \zeta_{2}-120 \zeta_{3}\right) C_{F}^{2} C_{A} \\
& +\left(\frac{6308}{27}+\frac{236}{3} \zeta_{2}-72 \zeta_{3}\right) C_{F} C_{A}^{2}+\left(\frac{272}{3}+\frac{32}{3} \zeta_{2}\right) C_{F}^{2} n_{f} \\
& +\left(\frac{620}{27}-\frac{56}{3} \zeta_{2}\right) C_{F} C_{A} n_{f}-\frac{232}{27} C_{F} n_{f}^{2}
\end{aligned}
$$

Thus already the leading large- $x$ terms are of a more complicated form in the off-diagonal cases.

At small- $x$ these physical evolution kernels, unlike the $\overline{\mathrm{MS}}$ splitting functions [49], include double-logarithmic $1 / x$-contributions originating, via Eqs. (2.25) and (2.27), in the corresponding behaviour for the coefficient functions $c_{\phi, \mathrm{p}}^{(n)}$ discussed in the previous section. Specifically, the quantities $K_{\phi 2}^{(n)}(x)$ and $K_{\phi \phi}^{(n)}(x)$ are found to include terms up to $x^{-1} \ln ^{2 n-1} x$ for $n=1,2,3$. The corresponding leading small- $x$ contributions to the $\mathrm{N}^{2} \mathrm{LO}$ and $\mathrm{N}^{3} \mathrm{LO}$ kernels $K_{22}^{(n)}(x)$ and $K_{2 \phi}^{(n)}(x)$ are of the form $x^{-1} \ln ^{2 n-2} x$. Since also these terms are artifacts of the large- $m_{\text {top }}$ approximation discussed above, we will not write them down here. 
Instead we directly proceed to a brief discussion of the numerical implications of Eqs. (4.8) (4.12) and (B.1) - B.5). The resulting perturbative expansions (4.1) of the elements $K_{a b}$ of this evolution kernel are illustrated in Figs. 6 - 9. Here the initial conditions $F_{2}\left(x, Q_{0}^{2}\right)$ and $F_{\phi}\left(x, Q_{0}^{2}\right)$ are physical quantities and consequently, unlike the $\overline{\mathrm{MS}}$ parton densities in Section 3, in principle independent of the perturbative order. Hence it is easier here to provide a realistic (and not only schematic, as in the previous section) account of the perturbative (in-)stability of the quantities under consideration.

The only remaining order-dependent quantity is the $\overline{\mathrm{MS}}$ coupling at our chosen reference scale. Here we employ the NLO and NNLO values obtained from a schematic analysis of non-singlet scaling violation in Refs. $[9,56]$, supplemented by a corresponding LO result,

$$
\begin{aligned}
& \alpha_{\mathrm{s}}\left(Q_{0}^{2}\right)_{\mathrm{LO}}=0.255 \\
& \alpha_{\mathrm{s}}\left(Q_{0}^{2}\right)_{\mathrm{NLO}}=0.208 \\
& \alpha_{\mathrm{S}}\left(Q_{0}^{2}\right)_{\mathrm{NNLO}}=0.201
\end{aligned}
$$

These values are consistent with a NLO - NNLO difference of about 0.002 to 0.003 at the mass of the $Z$-boson as found in recent fits of structure functions and related data [57]. The actual scale $Q_{0}^{2}$ in Eq. (4.29) depends, of course, on the value of $\alpha_{\mathrm{s}}\left(M_{Z}^{2}\right)$ with, at present, $20 \mathrm{GeV}^{2} \lesssim Q_{0}^{2} \lesssim 50 \mathrm{GeV}^{2}$.

For the illustrations of the resulting scaling violations in Figs. 10 and 11 we use the sufficiently characteristic form (3.51) for the structure functions at the reference scale $Q_{0}^{2}$, i.e., we employ

$$
\begin{aligned}
& F_{2}\left(x, Q_{0}^{2}\right)=0.6 x^{-0.3}(1-x)^{3.5}\left(1+5.0 x^{0.8}\right), \\
& F_{\phi}\left(x, Q_{0}^{2}\right)=1.6 x^{-0.3}(1-x)^{4.5}\left(1-0.6 x^{0.3}\right) .
\end{aligned}
$$

Note that, as implied by the identity of Eqs. (3.51) and (4.30), we have absorbed the average $\left\langle e^{2}\right\rangle$ of the quark charges into the definition of the singlet structure function $F_{2}$, which is thus given at leading order by $F_{2}=x q_{\mathrm{s}}$ instead of the standard normalization $F_{2}=\left\langle e^{2}\right\rangle x q_{\mathrm{s}}$.

At medium to large $x$ especially the diagonal entries $K_{22}$ and $K_{\phi \phi}$, respectively shown in Figs. 6 and 7, are rather stable, with the relative NNLO corrections not exceeding $10 \%$ in the range $0.2<$ $x<0.9$ in both cases. Larger higher-order effects are found in this region for the (absolutely smaller) off-diagonal kernels displayed in Figs. 8 and 9. In all cases the perturbative expansion appears ill-behaved at small values of $x$, starting from about $x \simeq 10^{-1}$ for $K_{\phi \phi}$ to $x \simeq 10^{-3}$ for $K_{\phi 2}$. We expect that a (physically realistic) inclusion of the finite- $m_{\text {top }}$ effects as discussed below Eq. (3.49) would fundamentally change these small- $x$ results.

Due to the ubiquitous Mellin convolutions (3.50) - and the diagonal distribution effects not visible in Figs. 6 and 7 - even the present evolution of the structure functions $F_{2}$ and $F_{\phi}$, illustrated in Figs. 10 and 11, is perfectly stable down to about $x=10^{-2}$ and rather acceptable at $x$-values as low as $x \simeq 10^{-3}$. At large $x$ these physical scaling violations are, unsurprisingly, much stronger (by a factor between 2 and 3) for the gluonic observable $F_{\phi}$ than for $F_{2}$. Here the relative NNLO corrections exceed $5 \%$ only at $x>0.7$ for the scale derivative of $F_{2}$, and at $x \geq 0.6$ for that of $F_{\phi}$. 

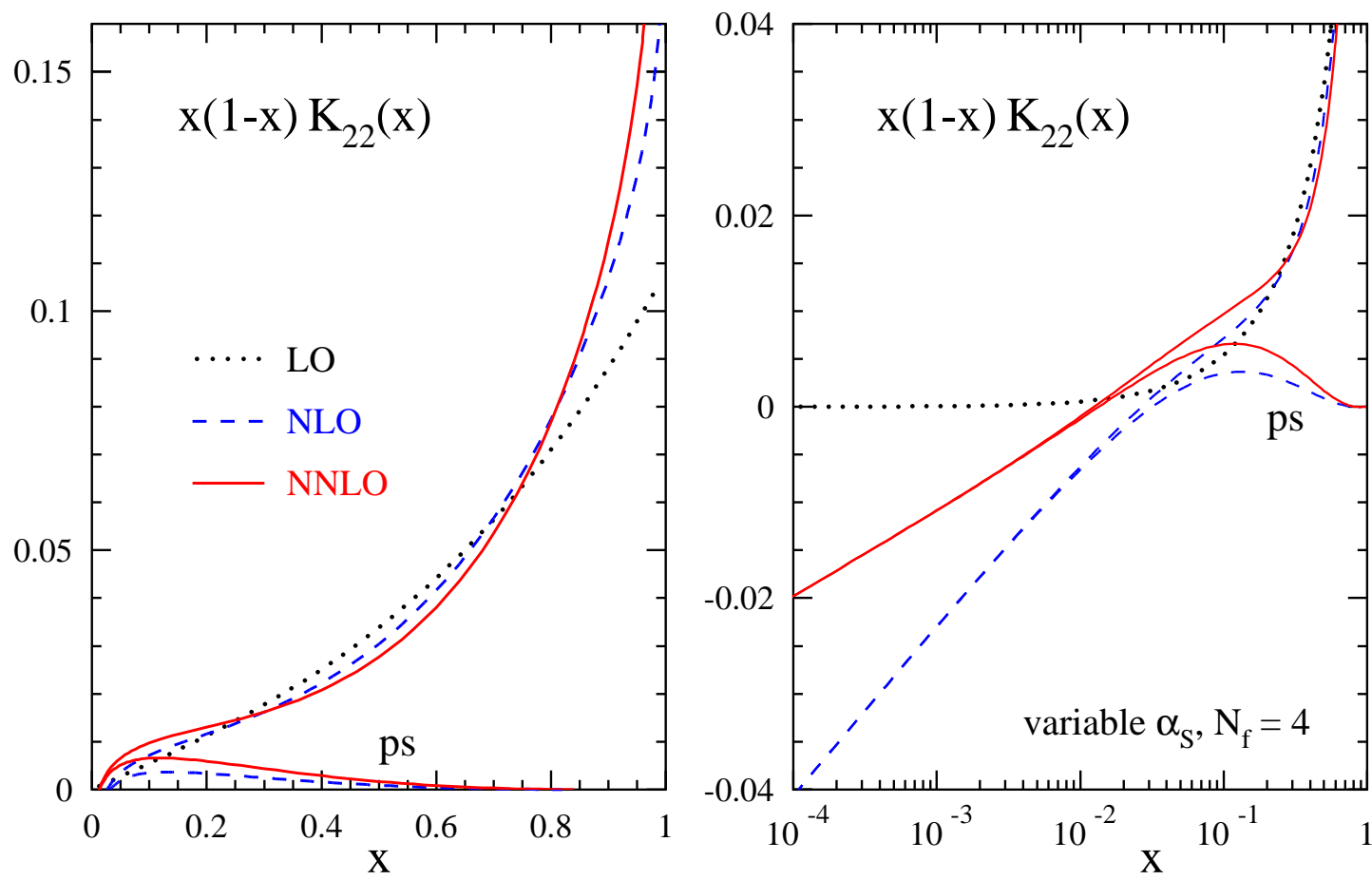

Figure 6: The perturbative expansion to order $\alpha_{\mathrm{s}}^{3}$ (NNLO) of the diagonal element $K_{22}(x)$ of the physical evolution-kernel matrix for the system $\left(F_{2}, F_{\phi}\right)$ of flavour-singlet DIS structure functions at $x<1$. The poles at $x=0$ and $x=1$ have been suppressed by multiplication with $x(1-x)$. Also shown is the pure-singlet contribution $K_{22, \mathrm{ps}}$ defined in Eq. (4.2).
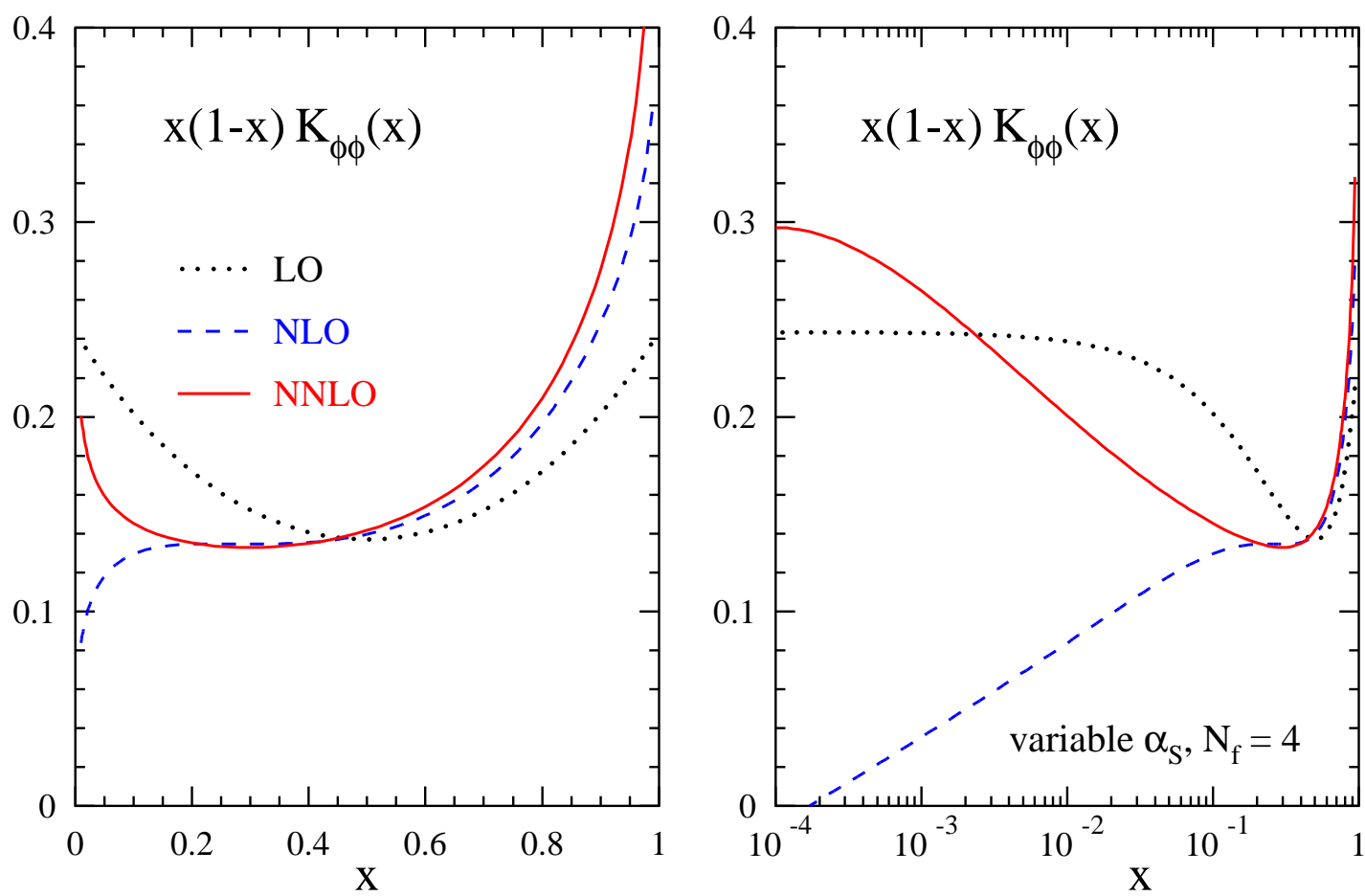

Figure 7: As Fig. 6, but for the second diagonal element $K_{\phi \phi}(x)$. As in all figures in this section, the order-dependent values (4.29) are employed for the strong coupling $\alpha_{\mathrm{s}}$. 

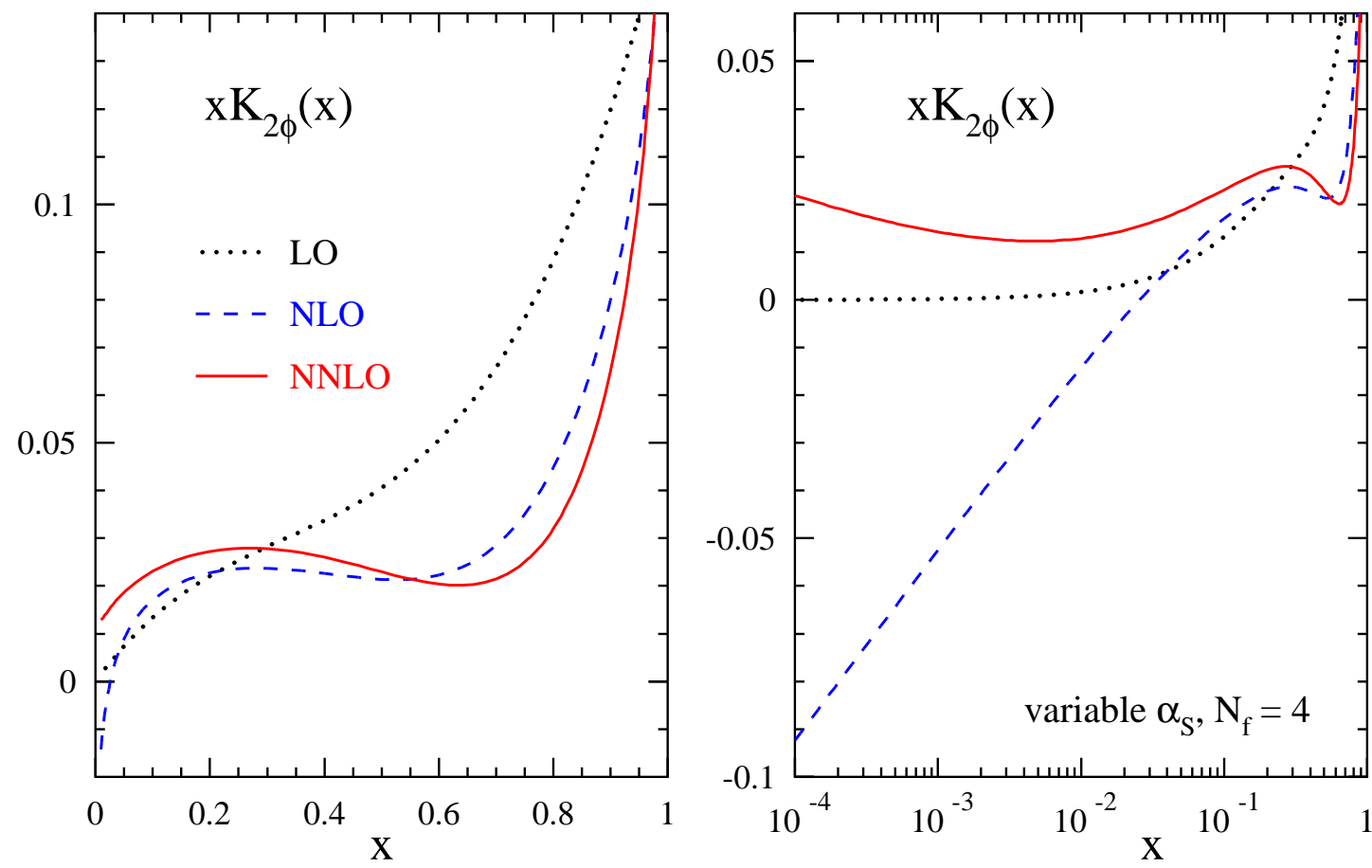

Figure 8: The perturbative expansion to order $\alpha_{\mathrm{s}}^{3}$ (NNLO) of the off-diagonal element $K_{2 \phi}(x)$ of the physical evolution-kernel matrix for the system $\left(F_{2}, F_{\phi}\right)$ of singlet structure functions.
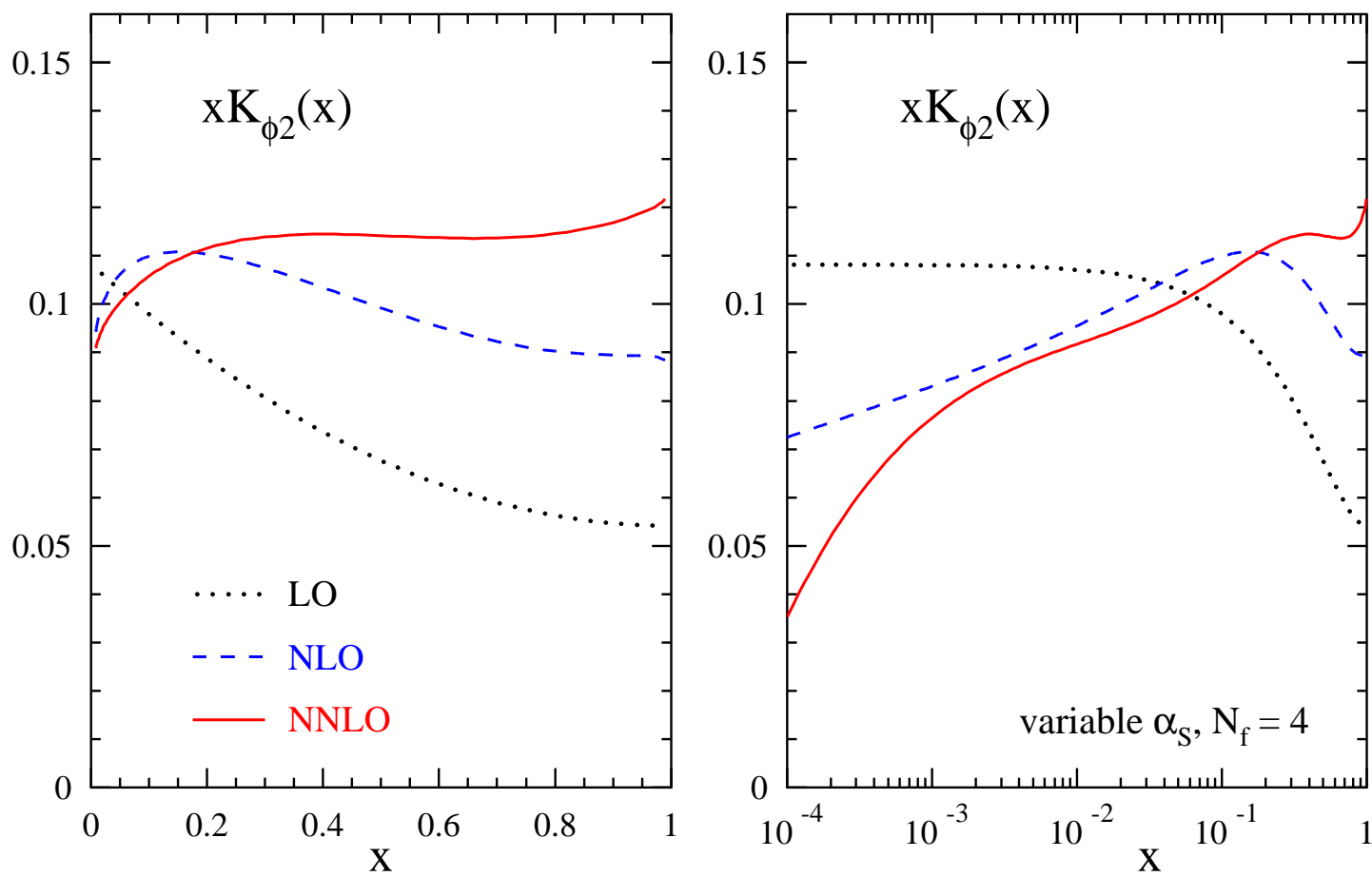

Figure 9: As Fig. 8 , but for the second off-diagonal element $K_{\phi 2}(x)$. In both cases the curves have multiplied by a factor of $x$ compensating the main $x$-dependence. 

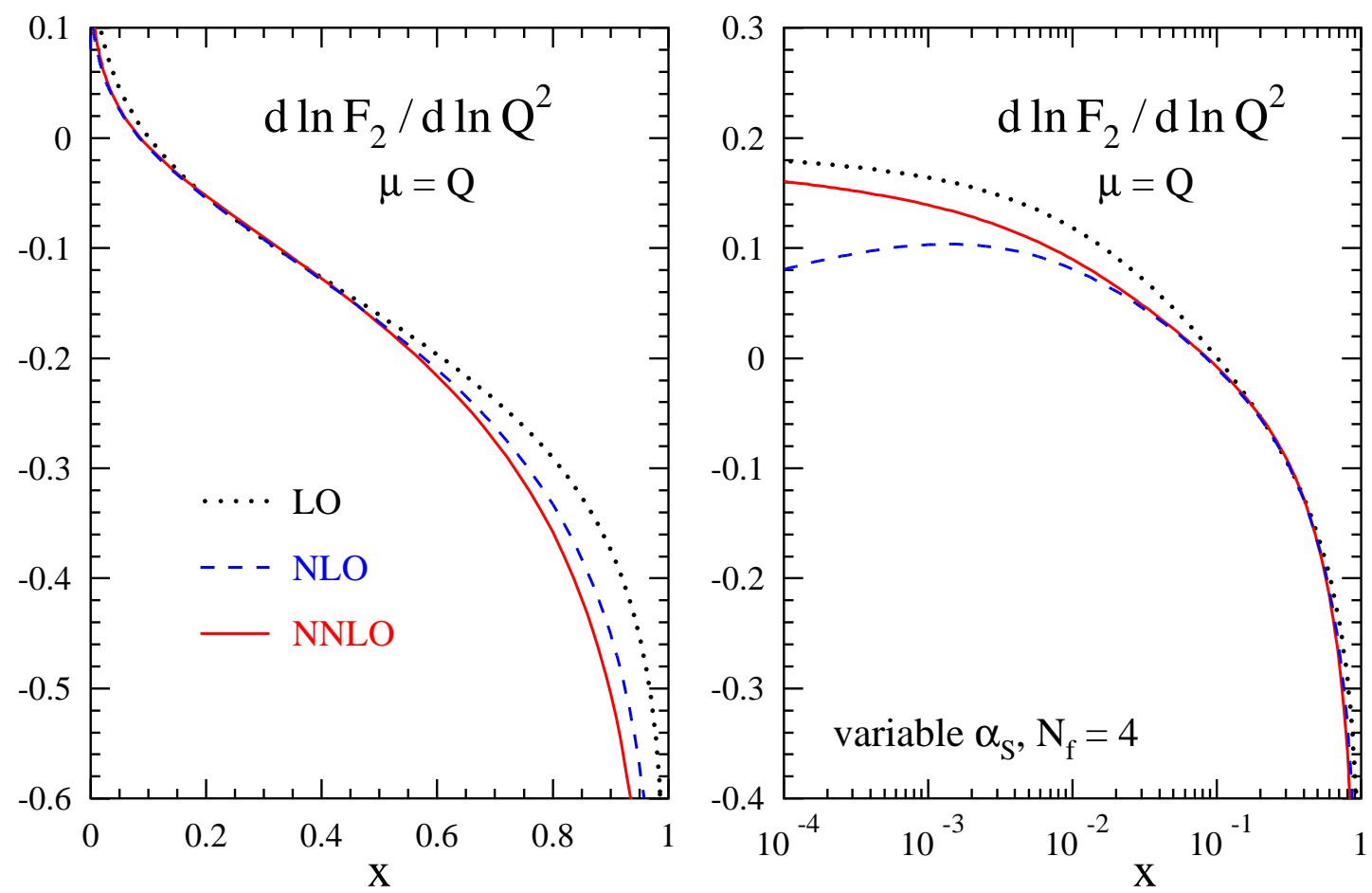

Figure 10: The perturbative expansion (2.28) to $l=2$ (NNLO) of the normalized scale derivative $d \ln F_{2} / d \ln Q^{2}$ of the photon-exchange singlet structure function $F_{2}$ for the initial conditions (4.30) at a mid-scale reference point with the coupling constants 4.29).
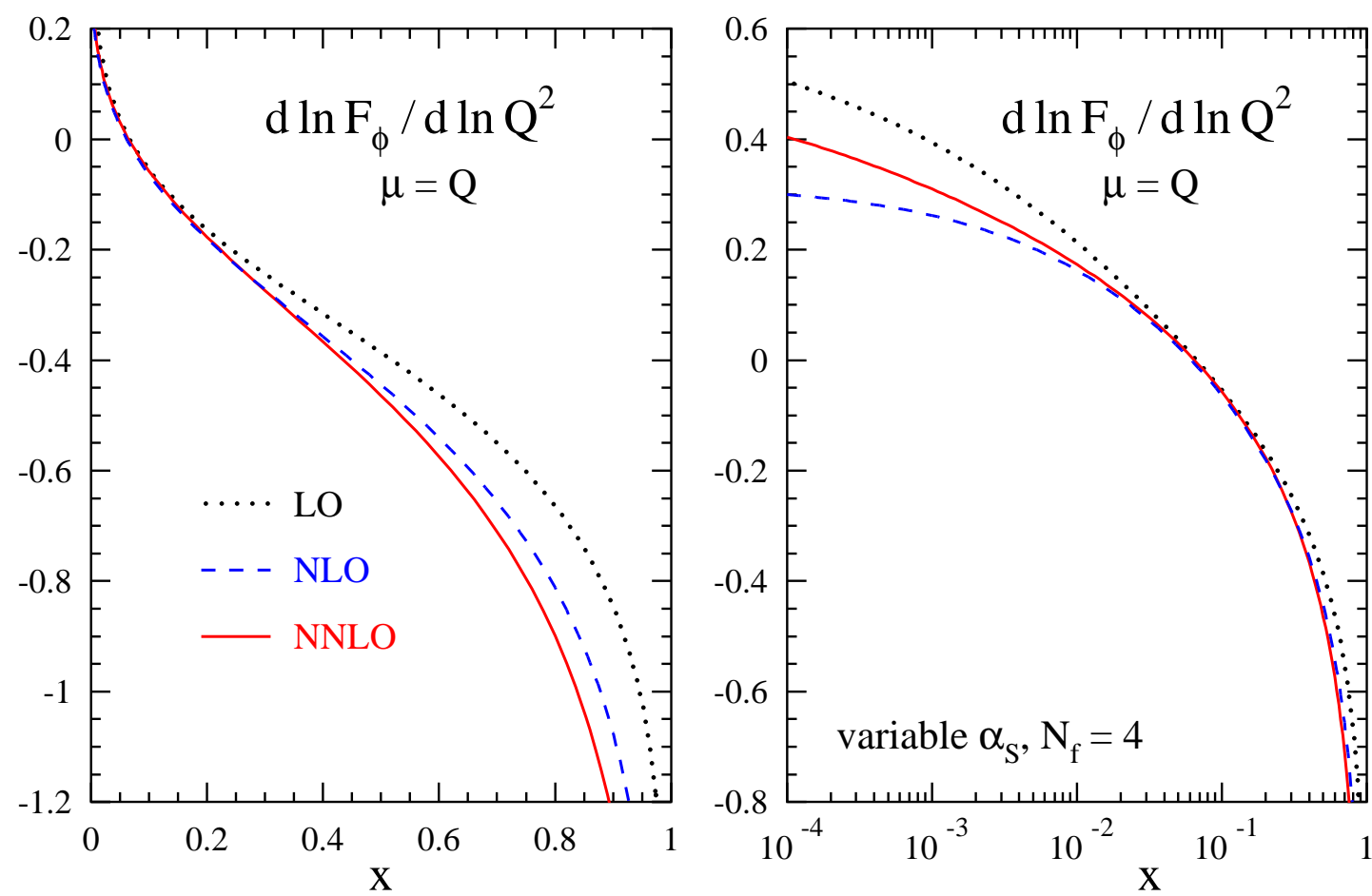

Figure 11: As Fig. 10, but for the scaling violations of the scalar-exchange structure function $F_{\phi}$. 


\section{Large- $x$ behaviour and four-loop predictions}

We now return to the expansion of the physical kernels and the $\overline{\mathrm{MS}}$ splitting functions in powers of $L_{1} \equiv \ln (1-x)$. The leading large- $x$ terms, $(1-x)^{-1} L_{1}^{k}$ for $K_{22}$ and $K_{\phi \phi}$ and $(1-x)^{0} L_{1}^{k}$ for $K_{2 \phi}$ and $K_{\phi 2}$, have been given to order $\alpha_{\mathrm{s}}^{3}$ (NNLO) in Eqs. (4.13) - (4.28). In fact, the only singlelogarithmic enhancement $-k \leq n$ at $\mathrm{N}^{n} \mathrm{LO}$ - does not only hold for these leading contributions, but to all orders in $(1-x)$ for all matrix entries. Thus the physical evolution kernels fulfill

$$
\left.K_{a b}^{(n)}\right|_{L_{1}^{n+k} f(x)}=0 \quad \text { for } \quad k \geq 1
$$

to (at least) $n=2$, where $f(x)$ denotes functions with a non-logarithmic expansion at $x=1$.

Already the leading $L_{1}^{n} f(x)$ terms are not particularly simple for the off-diagonal evolution kernels, hence we refrain from writing them down here for brevity. The observation made below Eq. (4.22) for the diagonal entries, on the other hand, also generalizes to all orders in $(1-x)$, i.e., the leading terms are those of the non-singlet kernels, and therefore given by [20]

$$
\begin{aligned}
& K_{22}^{(1)}(x)=\ln (1-x) p_{\mathrm{qq}}(x)\left[-2 C_{F} \beta_{0}-8 C_{F}^{2} \mathrm{H}_{0}\right]+o\left(\ln ^{0}(1-x)\right) \\
& K_{22}^{(2)}(x)=\ln ^{2}(1-x) p_{\mathrm{qq}}(x)\left[2 C_{F} \beta_{0}^{2}+12 C_{F}^{2} \beta_{0} \mathrm{H}_{0}+32 C_{F}^{3} \mathrm{H}_{0,0}\right]+o(\ln (1-x))
\end{aligned}
$$

and

$$
\begin{aligned}
& K_{\phi \phi}^{(1)}(x)=\ln (1-x) 2 p_{\mathrm{gg}}(x)\left[-2 C_{A} \beta_{0}-8 C_{A}^{2} \mathrm{H}_{0}\right]+o\left(\ln ^{0}(1-x)\right), \\
& K_{\phi \phi}^{(2)}(x)=\ln ^{2}(1-x) 2 p_{\mathrm{gg}}(x)\left[2 C_{A} \beta_{0}^{2}+12 C_{A}^{2} \beta_{0} \mathrm{H}_{0}+32 C_{A}^{3} \mathrm{H}_{0,0}\right]+o(\ln (1-x)) .
\end{aligned}
$$

Note that the additional factor of 2 in the latter two equations arises from a mismatch, in terms of the normalization of the leading $(1-x)^{-1}$ terms, of the definitions of $p_{\mathrm{qq}}(x)$ and $p_{\mathrm{gg}}(x)$ in Eqs. (3.7) and (4.7). These results imply that even the $L_{1}^{n} f(x)$ contributions to the pure-singlet quark and gluon kernels (recall the discussion in the paragraph above Eq. (3.22) ) vanish to at least $n=2$,

$$
\left.K_{22, \mathrm{ps}}^{(n)}\right|_{L_{1}^{n} f(x)}=\left.K_{\phi \phi}^{(n)}\right|_{C_{F} L_{1}^{n} f(x)}=0
$$

The above behaviour of the physical evolution kernels is in striking contrast to that of the $\overline{\mathrm{MS}}$-scheme splitting functions, which do include double-logarithmic contributions, $\ln ^{k}(1-x)$ with $k>n \geq 1$ at $\mathrm{N}^{n} \mathrm{LO}$, at all orders in the expansion at $x=1$. At NLO these contributions, and the single-logarithmic terms of the diagonal splitting functions, are given by

$$
\begin{aligned}
& P_{\mathrm{qg}}^{(1)}(x)=\ln ^{2}(1-x) C_{A F} n_{f}\left\{-4 p_{\mathrm{qg}}(x)\right\}+o(\ln (1-x)), \\
& P_{\mathrm{gq}}^{(1)}(x)=\ln ^{2}(1-x) C_{A F} C_{F}\left\{+4 p_{\mathrm{gq}}(x)\right\}+O(\ln (1-x))
\end{aligned}
$$

with $C_{A F} \equiv C_{A}-C_{F}$, and

$$
\begin{aligned}
& P_{\mathrm{qq}}^{(1)}(x)=\ln (1-x) C_{F}^{2}\left\{-8 p_{\mathrm{qq}}(x)\right\} \mathrm{H}_{0}+o\left(\ln (1-x)^{0}\right), \\
& P_{\mathrm{gg}}^{(1)}(x)=\ln (1-x) C_{A}^{2}\left\{-16 p_{\mathrm{qq}}(x)\right\} \mathrm{H}_{0}+o\left(\ln (1-x)^{0}\right) .
\end{aligned}
$$


The corresponding NNLO results read

$$
\begin{aligned}
P_{\mathrm{qg}}^{(2)}(x)= & \ln ^{4}(1-x) C_{A F}^{2} n_{f} 4 / 3 p_{\mathrm{qg}}(x) \\
+ & \ln ^{3}(1-x)\left[C_{A F}^{2} n_{f}\left\{-\left(34+16 / x-92 x+64 x^{2}\right) / 9-8 / 3(1+4 x) \mathrm{H}_{0}\right\}\right. \\
& +C_{A F} C_{F} n_{f}\left\{-40 / 9-16 /(9 x)-28 / 9 x+80 / 9 x^{2}+\left(4-24 x+32 / 3 x^{2}\right) \mathrm{H}_{0}\right\} \\
& \left.+C_{A F} n_{f}^{2} 4 / 9 p_{\mathrm{qg}}(x)\right]+O\left(\ln ^{2}(1-x)\right) \\
P_{\mathrm{gq}}^{(2)}(x)= & \ln ^{4}(1-x) C_{A F}^{2} C_{F} 4 / 3 p_{\mathrm{gq}}(x) \\
+ & \ln ^{3}(1-x)\left[C_{A F}^{2} C_{F}\left\{\left(344 / x-316+170 x-16 x^{2}\right) / 9-8 / 3(4 / x-8+x) \mathrm{H}_{0}\right\}\right. \\
& +C_{A F} C_{F}^{2}\left\{128 /(9 x)-76 / 9-16 / 9\left(x+x^{2}\right)-(32 /(3 x)-24+4 x) \mathrm{H}_{0}\right\} \\
& \left.+C_{A F} C_{F} n_{f}\left(-20 / 9 p_{\mathrm{gq}}(x)\right)\right]+o\left(\ln ^{2}(1-x)\right)
\end{aligned}
$$

and

$$
\begin{aligned}
P_{\mathrm{qq}}^{(2)}(x)= & \ln ^{3}(1-x)\left[C_{A F} C_{F} n_{f}\left\{-32 /(9 x)-8 / 3+8 / 3 x+32 / 9 x^{2}-16 / 3(1+x) \mathrm{H}_{0}\right\}\right] \\
+ & \ln ^{2}(1-x)\left[C_{F}^{2} n_{f}\left\{2 / 3\left(16 / x+7-7 x-16 x^{2}\right)+8\left(3+4 x+4 / 3 x^{2}\right) \mathrm{H}_{0}\right\}\right. \\
& +C_{F} C_{A} n_{f}\left\{-8 /(9 x)-6+6 x+8 / 9 x^{2}+4 / 3\left(4 / x-11-17 x-4 x^{2}\right) \mathrm{H}_{0}\right\} \\
& +C_{F} n_{f}^{2}\left\{16 /(9 x)+4 / 3-4 / 3 x-16 / 9 x^{2}+8 / 3(1+x) \mathrm{H}_{0}\right\} \\
& \left.+C_{A F} C_{F} n_{f}\left\{8(1+x)\left(\mathrm{H}_{0,0}-2 \widetilde{\mathrm{H}}_{1,0}\right)\right\}\right]+C_{F}^{3} 32 p_{\mathrm{qq}}(x) \mathrm{H}_{0,0} \\
+ & O(\ln (1-x)) \\
P_{\mathrm{gg}}^{(2)}(x)= & \ln ^{3}(1-x)\left[C_{A F} C_{F} n_{f}\left\{32 /(9 x)+8 / 3-8 / 3 x-32 / 9 x^{2}+16 / 3(1+x) \mathrm{H}_{0}\right\}\right] \\
+ & \ln ^{2}(1-x)\left[C_{F}^{2} n_{f}\left\{-2 / 3\left(16 / x+7-7 x-16 x^{2}\right)-8\left(3+4 x+4 / 3 x^{2}\right) \mathrm{H}_{0}\right\}\right. \\
& +C_{F} C_{A} n_{f}\left\{8 /(9 x)+6-6 x-8 / 9 x^{2}-4 / 3\left(4 / x-11-17 x-4 x^{2}\right) \mathrm{H}_{0}\right\} \\
& +C_{F} n_{f}^{2}\left\{-16 /(9 x)-4 / 3+4 / 3 x+16 / 9 x^{2}-8 / 3(1+x) \mathrm{H}_{0}\right\} \\
& \left.+C_{A F} C_{F} n_{f}\left\{-8(1+x)\left(\mathrm{H}_{0,0}-2 \widetilde{\mathrm{H}}_{1,0}\right)\right\}\right]+C_{A}^{3} 64 p_{\mathrm{gg}}(x) \mathrm{H}_{0,0} \\
+ & O(\ln (1-x)) .
\end{aligned}
$$

The respective final terms of the last two equations represent the non-singlet contributions directly corresponding to the final terms in Eqs. (5.3) and (5.5).

Eqs. (5.7) - 5.14 show a couple of interesting features: The double-logarithmic contributions, unlike the single-logarithmic terms, all vanish for the case $C_{F}=C_{A}$ which forms a subset of the colour factor conditions leading to an $\mathcal{N}=1$ supersymmetric theory. In fact, the coefficients of the leading, next-to-leading etc double logarithms successively include terms of the form $\left(C_{A}-C_{F}\right)^{n-1},\left(C_{A}-C_{F}\right)^{n-2}$ etc. Moreover, to the accuracy of Eqs. (5.13) and (5.14), the puresinglet parts of $P_{\mathrm{qq}}^{(2)}$ and $P_{\mathrm{gg}}^{(2)}$ turn out to be completely identical up to an overall sign. 
Both the single logarithmic enhancement of the physical kernels also in the present flavoursinglet case, and the above features are non-trivial (and not obviously related) properties. In the non-singlet case the former property is known to hold also at $\mathrm{N}^{3} \mathrm{LO}$ [20]. Recall also that it is implied [56] for the leading $(1-x)^{-1}$ by the soft-gluon exponentiation [21], and established at all powers of $(1-x)$ in the large- $n_{f}$ limit by Refs. $[58,59]$.

Hence it appears very natural to conjecture that also Eqs. (5.1) and Eqs. (5.6) are valid at $\mathrm{N}^{3} \mathrm{LO}$ as well. As the three-loop coefficient functions are known from Refs. [9] and Section 3, this implies that the known double logarithms originating from the matrix (2.27) in Eq. (2.25) at order $\alpha_{\mathrm{s}}^{4}$ have to be compensated by the (hitherto unknown) corresponding contributions to the fourloop splitting functions $P^{(3)}$. These terms can therefore be predicted from the above conjecture, and checked against the colour-factor pattern expected from Eqs. (5.7) - (5.14).

For the off-diagonal splitting functions these predictions are found to be

$$
\begin{aligned}
& P_{\mathrm{qg}}^{(3)}(x)=\ln ^{5}(1-x)\left[C_{A F}^{3} n_{f}\left\{32 /(27 x)+46 / 27+52 / 27 x-4 x^{2}+16 / 9(1+4 x) \mathrm{H}_{0}\right\}\right. \\
&+ C_{A F}^{2} C_{F} n_{f}\left\{32 /(27 x)+58 / 27+100 / 27 x-68 / 9 x^{2}+8 / 9(1+10 x) \mathrm{H}_{0}\right\} \\
&-\left.C_{A F}^{2} n_{f}^{2}\left\{4 / 27 p_{\mathrm{qg}}(x)\right\}\right] \\
&+ \ln ^{4}(1-x)\left[C _ { A F } ^ { 3 } n _ { f } \left\{152 /(27 x)-106 / 9+1178 / 27 x-719 / 27 x^{2}\right.\right. \\
&-2 / 9\left(8 /(3 x)-1+16 x-1114 / 3 x^{2}\right) \mathrm{H}_{0}-80 / 9\left(1+2 x+2 x^{2}\right) \widetilde{\mathrm{H}}_{-1,0} \\
&\left.-(104 / 9+160 / 9 x) \mathrm{H}_{0,0}+8 / 9\left(11+38 x+2 x^{2}\right) \widetilde{\mathrm{H}}_{1,0}-80 / 9 \zeta_{2} p_{\mathrm{qg}}(x)\right\} \\
&+ C_{A F}^{2} C_{F} n_{f}\left\{-176 /(81 x)+685 / 162-1426 / 81 x+1166 / 27 x^{2}\right. \\
&-1 / 27\left(32 / x-71+742 x-2156 x^{2}\right) \mathrm{H}_{0}-80 / 9\left(1+2 x+2 x^{2}\right) \widetilde{\mathrm{H}}_{-1,0} \\
&\left.+8 / 9\left(31-2 x+52 x^{2}\right) \widetilde{\mathrm{H}}_{1,0}+16 / 9\left(7 x+10 x^{2}\right) \mathrm{H}_{0,0}-8 \zeta_{2} p_{\mathrm{qg}}(x)\right\} \\
&+ C_{A F}^{2} n_{f}^{2}\left\{8 /(27 x)-98 / 81+184 / 81 x-226 / 81 x^{2}+4 / 9(1+4 x) \mathrm{H}_{0}\right\} \\
&+ C_{A F} C_{F}^{2} n_{f}\left\{-632 /(81 x)-877 / 81+1349 / 81 x+49 / 27 x^{2}\right. \\
&\left.-1 / 27\left(16 / x+211+28 x+552 x^{2}\right) \mathrm{H}_{0}-\left(10-220 / 3 x+80 / 3 x^{2}\right) \mathrm{H}_{0,0}\right\} \\
&+ C_{A F} C_{F} n_{f}^{2}\left\{-40 /(81 x)+719 / 81-1060 / 81 x+398 / 81 x^{2}\right. \\
&\left.+2 / 27\left(29+50 x-52 x^{2}\right) \mathrm{H}_{0}+8 / 9(2-29 x) \mathrm{H}_{0,0}\right\} \\
&-\left.C_{A F} n_{f}^{3}\left\{4 / 81 p_{\mathrm{qg}}(x)\right\}\right]+o\left(\ln ^{3}(1-x)\right) \\
&
\end{aligned}
$$

and

$$
\begin{aligned}
P_{\mathrm{gq}}^{(3)}(x)= & \ln ^{5}(1-x)\left[C_{A F}^{3} C_{F}\left\{\left(292 / x-236+46 x-32 x^{2}\right) / 27+16 / 9(4+x) \mathrm{H}_{0}\right\}\right. \\
& +C_{A F}^{2} C_{F}^{2}\left\{\left(148 / x-44-86 x-32 x^{2}\right) / 27+8 / 9(10+x) \mathrm{H}_{0}\right\} \\
& \left.-C_{A F}^{2} C_{F} n_{f}\left\{4 / 27 p_{\mathrm{gq}}(x)\right\}\right]
\end{aligned}
$$




$$
\begin{aligned}
+ & \ln ^{4}(1-x)\left[C _ { A F } ^ { 3 } C _ { F } \left\{3029 /(81 x)-428 / 81+295 / 81 x+128 / 27 x^{2}\right.\right. \\
& -\left(1168 / x-2300-68 x+176 x^{2}\right) / 27 \mathrm{H}_{0}+(80 /(3 x)-400 / 9+16 x) \mathrm{H}_{0,0} \\
& \left.+80 / 9(2 / x+2+x) \widetilde{\mathrm{H}}_{-1,0}+8 / 9(26 / x+14+23 x) \widetilde{\mathrm{H}}_{1,0}+16 / 9 \zeta_{2} p_{\mathrm{gq}}(x)\right\} \\
+ & C_{A F}^{2} C_{F}^{2}\left\{7142 /(81 x)-1418 / 27-505 / 54 x+736 / 81 x^{2}-(1472 /(27 x)\right. \\
& \left.-1022 / 9-89 / 9 x+64 / 9 x^{2}\right) \mathrm{H}_{0}+(160 /(3 x)-128+280 / 9 x) \mathrm{H}_{0,0} \\
& \left.+80 / 9(2 / x+2+x) \widetilde{\mathrm{H}}_{-1,0}+8 / 9(52 / x-2+31 x) \widetilde{\mathrm{H}}_{1,0}-8 \zeta_{2} p_{\mathrm{gq}}(x)\right\} \\
+ & C_{A F}^{2} C_{F} n_{f}\left\{-2290 /(81 x)+656 / 27-70 / 9 x+184 / 81 x^{2}\right. \\
& \left.+(8 /(3 x)-440 / 27-56 / 27 x) \mathrm{H}_{0}\right\} \\
+ & C_{A F} C_{F}^{3}\left\{47 /(3 x)-1855 / 81+185 / 81 x+352 / 81 x^{2}\right. \\
& \left.-\left(304 / x-424+17 x+16 x^{2}\right) / 27 \mathrm{H}_{0}+(80 /(3 x)-220 / 3+10 x) \mathrm{H}_{0,0}\right\} \\
+ & C_{A F} C_{F}^{2} n_{f}\left\{-\left(1474 / x-1460+217 x-248 x^{2}\right) / 81\right. \\
& \left.+(8 / x-532+62 x) / 27 \mathrm{H}_{0}+16 / 9(2-x) \mathrm{H}_{0,0}\right\} \\
+ & \left.C_{A F} C_{F} n_{f}^{2}\left\{32 / 81 p_{\mathrm{gq}}(x)\right\}\right]+o\left(\ln ^{3}(1-x)\right) .
\end{aligned}
$$

Here, and in Eqs. (5.19) and (5.20) below, we have employed the modified basis of Ref. [20] for the harmonic polylogarithms, in which all logarithms and $\zeta$-functions are made explicit in the expansion at $x=1$ to all orders. The corresponding functions entering the above expression are

$$
\widetilde{\mathrm{H}}_{-1,0}(x)=\mathrm{H}_{-1,0}(x)+\zeta_{2} / 2, \quad \widetilde{\mathrm{H}}_{1,0}(x)=\mathrm{H}_{1,0}(x)+\zeta_{2}
$$

and, e.g., $\mathrm{H}_{0,1}(x)$ has been expressed by

$$
\mathrm{H}_{0,1}(x)=-\widetilde{\mathrm{H}}_{1,0}(x)-\ln (1-x) \mathrm{H}_{0}(x)-\zeta_{2} .
$$

The corresponding predictions for the four-loop pure-singlet splitting functions (which, as expected, are suppressed by another power of $(1-x)$ for $x \rightarrow 1)$ read

$$
\begin{aligned}
& P_{\mathrm{qq}, \mathrm{ps}}^{(3)}(x)=\ln ^{4}(1-x)\left[C _ { A F } ^ { 2 } C _ { F } n _ { f } \left\{-728 /(81 x)-602 / 27+122 / 27 x+2168 / 81 x^{2}\right.\right. \\
&\left.+4 / 27\left(40 / x-121-241 x-40 x^{2}\right) \mathrm{H}_{0}+80 / 9(1+x) \mathrm{H}_{0,0}\right\} \\
&+ C_{A F} C_{F}^{2} n_{f}\left\{-248 /(81 x)-62 / 27+62 / 27 x+248 / 81 x^{2}\right. \\
&\left.+4 / 27\left(40 / x-1-61 x-40 x^{2}\right) \mathrm{H}_{0}+160 / 9(1+x) \mathrm{H}_{0,0}\right\} \\
&+\left.C_{A F} C_{F} n_{f}^{2}\left\{176 /(81 x)+44 / 27(1-x)-176 / 81 x^{2}+88 / 27(1+x) \mathrm{H}_{0}\right\}\right] \\
&+ \ln ^{3}(1-x)\left[C _ { F } ^ { 3 } n _ { f } \left\{1072 /(27 x)+610 / 9-2182 / 27 x-80 / 3 x^{2}\right.\right. \\
& \quad-\left(320 /(27 x)+80 / 9(1-x)-320 / 27 x^{2}\right) \zeta_{2}+(2252 / 27-884 / 27 x \\
&\left.\quad-2560 / 27 x^{2}-160 / 9 \zeta_{2}(1+x)\right) \mathrm{H}_{0}-8 / 3\left(5+25 / 3 x+152 / 9 x^{2}\right) \mathrm{H}_{0,0}
\end{aligned}
$$




$$
\begin{aligned}
& +\left(1216 /(27 x)-112 / 3-1264 / 9 x-1216 / 27 x^{2}\right) \widetilde{\mathrm{H}}_{1,0} \\
& \left.-320 / 9(1+x) \widetilde{\mathrm{H}}_{1,0,0}+32(1+x) \widetilde{\mathrm{H}}_{0,1,0}+560 / 9(1+x) \mathrm{H}_{0,0,0}\right\} \\
+ & C_{F}^{2} C_{A} n_{f}\left\{-24232 /(243 x)+8954 / 81-16730 / 81 x+47560 / 243 x^{2}\right. \\
& +\left(64 /(27 x)+16 / 9(1-x)-64 / 27 x^{2}\right) \zeta_{2}-(4864 /(81 x)+2584 / 27 \\
& \left.-4952 / 27 x-6944 / 81 x^{2}-32 / 9(1+x) \zeta_{2}\right) \mathrm{H}_{0}-16 / 27(142 \\
& +301 x) \mathrm{H}_{0,0}+\left(640 /(27 x)-160 / 9(1+x)+640 / 27 x^{2}\right) \widetilde{\mathrm{H}}_{-1,0} \\
& -16 / 27\left(88 / x-205-457 x-88 x^{2}\right) \widetilde{\mathrm{H}}_{1,0}-320 / 9(1-x) \widetilde{\mathrm{H}}_{0,-1,0} \\
& \left.-128 / 3(1+x) \widetilde{\mathrm{H}}_{0,1,0}-(176 / 3-208 / 9 x) \mathrm{H}_{0,0,0}\right\} \\
+ & C_{F} C_{A}^{2} n_{f}\left\{1580 /(27 x)-13028 / 81+23804 / 81 x-1724 / 9 x^{2}\right. \\
& +\left(256 /(27 x)+64 / 9(1-x)-256 / 27 x^{2}\right) \zeta_{2}+(2720 /(81 x)+632 / 27 \\
& \left.-3656 / 27 x+3872 / 81 x^{2}+128 / 9(1+x) \zeta_{2}\right) \mathrm{H}_{0}-(832 /(27 x)-1376 / 27 \\
& \left.-4064 / 27 x-128 / 9 x^{2}\right) \mathrm{H}_{0,0}-160 / 9\left(4 /(3 x)-1-x+4 / 3 x^{2}\right) \widetilde{\mathrm{H}}_{-1,0} \\
& +\left(64 /(9 x)-2272 / 27-3520 / 27 x-64 / 9 x^{2}\right) \widetilde{\mathrm{H}}_{1,0}+32 / 3(1+x) \widetilde{\mathrm{H}}_{0,1,0} \\
& \left.+320 / 9(1-x) \widetilde{\mathrm{H}}_{0,-1,0}+320 / 9(1+x) \widetilde{\mathrm{H}}_{1,0,0}-(32 / 9+256 / 3 x) \mathrm{H}_{0,0,0}\right\} \\
+ & C_{F}^{2} n_{f}^{2}\left\{-\left(1504 / x+1820-860 x-2464 x^{2}\right) / 81-(368 / 9+160 / 3 x\right. \\
+ & \left.\left.704 / 81 x^{2}\right) \mathrm{H}_{0}+16 / 27(1+x) \mathrm{H}_{0,0}-352 / 27(1+x) \widetilde{\mathrm{H}}_{1,0}\right\} \\
+ & C_{F} C_{A} n_{f}^{2}\left\{3280 /(243 x)+1472 / 81-512 / 81 x-6160 / 243 x^{2}\right. \\
& -\left(704 /(81 x)-832 / 27-448 / 9 x-704 / 81 x^{2}\right) \mathrm{H}_{0} \\
& \left.-32 / 27(13+10 x) \mathrm{H}_{0,0}+352 / 27(1+x) \widetilde{\mathrm{H}}_{1,0}\right\} \\
+ & \left.C_{F} n_{f}^{3}\left\{-128 /(81 x)-32 / 27(1-x)+128 / 81 x^{2}-64 / 27(1+x) \mathrm{H}_{0}\right\}\right] \\
\hline & \left.1 n^{2}(1-x)\right)
\end{aligned}
$$

and, in perfect agreement with the NNLO observation below Eq. 5.14),

$$
\left.P_{\mathrm{gg}}^{(3)}(x)\right|_{C_{F}}=-P_{\mathrm{qq}, \mathrm{ps}}^{(3)}(x)+O\left(\ln ^{2}(1-x) f(x)\right) .
$$

Eq. (5.19) includes three more modified basis functions,

$$
\begin{aligned}
\widetilde{\mathrm{H}}_{0,-1,0}(x) & =\mathrm{H}_{0,-1,0}(x)+\mathrm{H}_{0}(x) \zeta_{2} / 2+3 \zeta_{3} / 2, \\
\widetilde{\mathrm{H}}_{0,1,0}(x) & =\mathrm{H}_{0,1,0}(x)+\mathrm{H}_{0}(x) \zeta_{2}+2 \zeta_{3}, \\
\widetilde{\mathrm{H}}_{1,0,0}(x) & =\mathrm{H}_{1,0,0}(x)-\zeta_{3} .
\end{aligned}
$$

Both the full quark-quark and gluon-gluon splitting functions differ from the above results by a term with one unknown coefficient, respectively given by $\xi_{P^{(3)}} C_{F}^{4} \ln ^{3}(1-x) p_{\mathrm{qq}}(x) \mathrm{H}_{0,0,0}(x)$ 
and $\xi_{P^{(3)}} C_{A}^{4} \ln ^{3}(1-x) 2 p_{\mathrm{gg}}(x) \mathrm{H}_{0,0,0}(x)$, which directly enters the large- $x$ behaviour of the diagonal physical kernels, given by [20]

$$
\left.K_{22}^{(3)}\right|_{L_{1}^{3} f(x)}=p_{\mathrm{qq}}(x)\left[-2 C_{F} \beta_{0}^{3}-44 / 3 C_{F}^{2} \beta_{0}^{2} \mathrm{H}_{0}-64 C_{F}^{3} \beta_{0} \mathrm{H}_{0,0}+\xi_{P_{3}} C_{F}^{4} \mathrm{H}_{0,0,0}\right],
$$

and correspondingly

$$
\left.K_{\phi \phi}^{(3)}\right|_{L_{1}^{3} f(x)}=2 p_{\mathrm{gg}}(x)\left[-2 C_{A} \beta_{0}^{3}-44 / 3 C_{A}^{2} \beta_{0}^{2} \mathrm{H}_{0}-64 C_{A}^{3} \beta_{0} \mathrm{H}_{0,0}+\xi_{P_{3}} C_{A}^{4} \mathrm{H}_{0,0,0}\right] .
$$

Obviously the $\mathrm{N}^{3} \mathrm{LO}$ predictions (5.15) and (5.16), and the double-logarithmic part $\sim \ln ^{4}(1-x)$ of Eq. (5.19), exactly follow the colour-factor pattern expected from the NLO and NNLO results (as discussed below Eq. (5.14)). In our view this represents, for the time being, an acceptably strong check of these predictions and thus of the underlying conjecture about the four-loop physical kernel matrix for the structure functions $\left(F_{2}, F_{\phi}\right)$. Furthermore we expect that a formal proof of this property, presumably first for subleading the $(1-x)^{0} \ln ^{k}(1-x)$ terms corresponding to $N^{-1} \ln N$ contributions in Mellin- $N$ space, can be obtained using, for instance, the recent path-integral approach of Ref. [60] or soft-collinear effective theory (SCET) [61]. For other recent research into $N^{-1} \ln N$ contributions to hard processes see Refs. [62].

In fact, the $(1-x)^{0} \ln ^{k}(1-x)$ contributions to the four-loop off-diagonal splitting function form the part of the above predictions which can be expected to become phenomenologically relevant first. These terms can be obtained from Eqs. (5.15) and (5.16) by removing all $\widetilde{\mathrm{H}}$-functions (the expansions of which at $x=1$ generally start with $(1-x)^{m}$ with $\left.m \geq 1\right)$ and setting $x=1$ in the remaining terms. For the convenience of the reader we finally write down the resulting expressions,

$$
\begin{aligned}
P_{\mathrm{qg}}^{(3)}(x)= & \ln ^{6}(1-x) \cdot 0 \\
+ & \ln ^{5}(1-x)\left[\frac{22}{27} C_{A F}^{3} n_{f}-\frac{14}{27} C_{A F}^{2} C_{F} n_{f}-\frac{4}{27} C_{A F}^{2} n_{f}^{2}\right] \\
+ & \ln ^{4}(1-x)\left[\left(\frac{293}{27}-\frac{80}{9} \zeta_{2}\right) C_{A F}^{3} n_{f}+\left(\frac{4477}{162}-8 \zeta_{2}\right) C_{A F}^{2} C_{F} n_{f}\right. \\
& \left.-\frac{13}{81} C_{A F} C_{F}^{2} n_{f}-\frac{116}{81} C_{A F}^{2} n_{f}^{2}+\frac{17}{81} C_{A F} C_{F} n_{f}^{2}-\frac{4}{81} C_{A F} n_{f}^{3}\right] \\
+ & O\left(\ln ^{3}(1-x)\right) \\
P_{\mathrm{gq}}^{(3)}(x)= & \ln ^{6}(1-x) \cdot 0 \\
+ & \ln ^{5}(1-x)\left[\frac{70}{27} C_{A F}^{3} C_{F}-\frac{14}{27} C_{A F}^{2} C_{F}^{2}-\frac{4}{27} C_{A F}^{2} C_{F} n_{f}\right] \\
+ & \ln ^{4}(1-x)\left[\left(\frac{3280}{81}+\frac{16}{9} \zeta_{2}\right) C_{A F}^{3} C_{F}+\left(\frac{637}{18}-8 \zeta_{2}\right) C_{A F}^{2} C_{F}^{2}\right. \\
& \left.-\frac{49}{81} C_{A F} C_{F}^{3}-\frac{256}{27} C_{A F}^{2} C_{F} n_{f}+\frac{17}{81} C_{A F} C_{F}^{2} n_{f}+\frac{32}{81} C_{A F} C_{F} n_{f}^{2}\right] \\
+ & O\left(\ln ^{3}(1-x)\right) .
\end{aligned}
$$



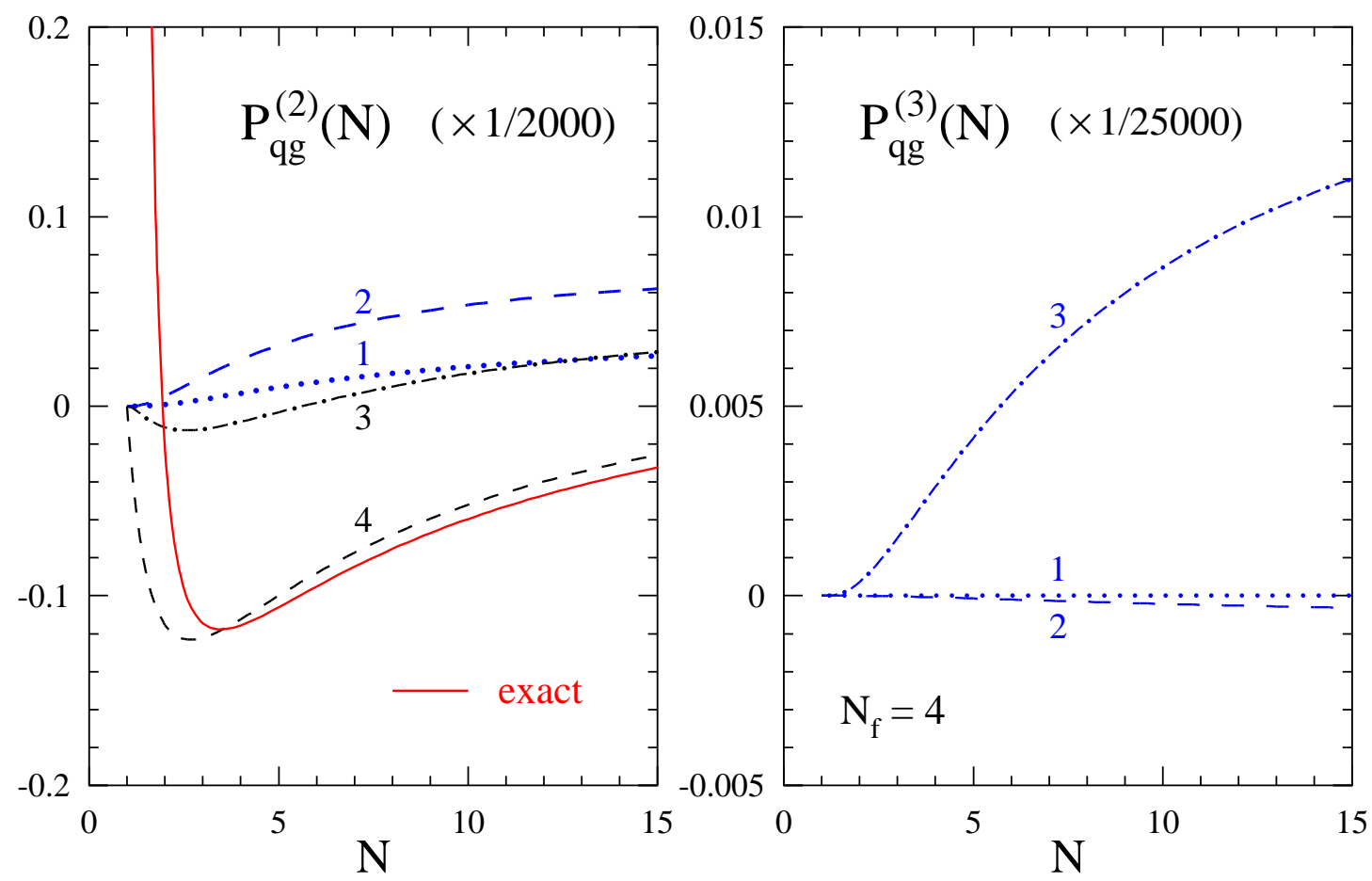

Figure 12: Large- $N$ expansions of the NNLO and $\mathrm{N}^{3} \mathrm{LO}$ gluon-quark splitting functions $P_{\mathrm{qg}}^{(2)}(N)$ and $P_{\mathrm{qg}}^{(3)}(N)$, in the former case compared with the exact result of Ref. [7]. The curves ' 1 ', ' 2 ', etc are successively including the leading logarithms, $N^{-1} \ln ^{4} N$ (left) and $N^{-1} \ln ^{6} N$ (right), next-toleading logarithms, $N^{-1} \ln ^{3} N$ (left) and $N^{-1} \ln ^{5} N$ (right), etc. All log-enhanced terms are shown at NNLO. Only the double-logarithmic contributions obtained in this article are available at $\mathrm{N}^{3} \mathrm{LO}$.
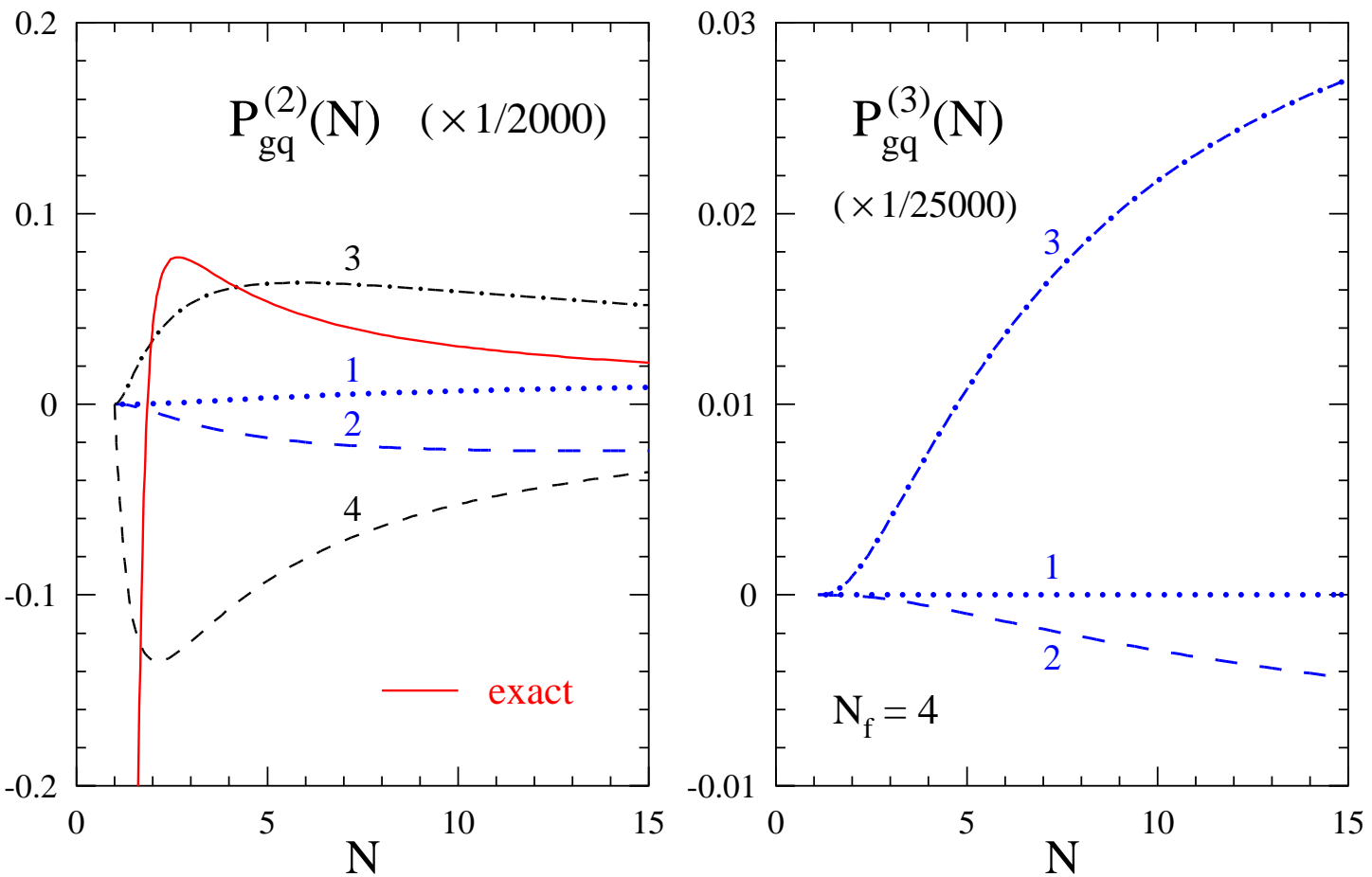

Figure 13: As Fig. 12, but for the NNLO (left) and $\mathrm{N}^{3} \mathrm{LO}$ (right) quark-gluon splitting functions. 
The only surprise in these results and Eqs. (5.15) and (5.16) is the absence, indicated explicitly in Eqs. (5.24 and (5.25), of the expected leading $\ln ^{6}(1-x)$ terms. In general the - related, recall Eqs. (5.7/8) and Eqs. (5.11/12) - coefficients of the leading $\ln ^{2 n}(1-x)$ terms of $P_{\mathrm{qg}}^{(3)}$ and $P_{\mathrm{gq}}^{(3)}$ receive contributions with both signs from Eq. (2.25), which happen to cancel at $n=3$. Such 'accidental' cancellations of leading coefficients at some orders do occur, for a famous example see Refs. [49], and do not indicate any problem. We expect a non-vanishing coefficient of $\ln ^{8}(1-x)$ at five loops. Analogous comments apply to the (presumably related) absence of the expected $\ln ^{5}(1-x)$ contribution to the diagonal fourth-order splitting functions in Eqs. (5.19) and (5.20).

The Mellin moments of Eqs. (5.24) and (5.25) and their NNLO counterparts in Eqs. (4.18) and (4.19) of Ref. [7] are numerically illustrated in Figs. 12 and 13. Unlike analogous results for the non-singlet coefficient functions in Refs. [19,20], even all $\ln N$ enhanced terms do not provide a meaningful approximation at relevant values of $N$ for $P_{\mathrm{gq}}^{(2)}$, and the double-logarithmic contributions (blue curves in the electronic version) are nowhere near the full results in either case. Hence our predicted double-log terms are not phenomenologically useful without further four-loop information, e.g., in the form of an extension of Refs. [23] to the next order. Indeed, given the fact that a first fourth-order calculation of a splitting-function moment has already been presented [63], such an extension should become feasible in the foreseeable future.

\section{Discussion and outlook}

It is an old idea to replace the factorization-scheme dependent NLO (and now NNLO) quark and gluon distributions by suitable reference observables. One may expect to obtain smaller higherorder corrections to the coefficient functions for other observables in such a scheme, having absorbed some universal corrections entering all coefficient functions in, e.g., the usual $\overline{\mathrm{MS}}$ scheme. The predictions of (massless) perturbative QCD then depend on only one unphysical scale and scheme, for the renormalized strong coupling $\alpha_{s}$. Moreover physical constraints on the chosen observables, for instance on their positivity or endpoint behaviour, would directly apply to the unavoidable non-perturbative initial distributions for the scale evolution in this case.

An obvious quantity to replace the overall (flavour-singlet) quark density is the (singlet part of the) standard structure function $F_{2}$ in (say, charged- current) deep-inelastic scattering, i.e., one can define physical quark densities $\tilde{q}_{\mathrm{S}}$ by $F_{2}=\tilde{q}_{\mathrm{S}}$ to NLO and NNLO. Any such scheme is usually called a DIS scheme [64]. Of course, this condition does not specify the lower row of the schemetransformation matrix $Z$ discussed in Section 2. The standard choice adopted in this respect, used in parton-distribution analyses mainly in the 1990s, was to arbitrarily use the coefficient functions for $F_{2}$ also for this lower row. Consequently the gluon distribution (which, in practical analyses, would profit most from being defined in terms of an observable) remains entirely unphysical in this version of the DIS scheme, which is therefore mostly useful for quark-dominated observables such as Drell-Yan lepton-pair production in $p \bar{p}$-collisions not too far from the threshold. 
Various alternatives to the above arbitrary choice - which, given that gluon-dominated quantities usually receive larger higher-order corrections than $F_{2}$, 'under-transforms' the gluon density have also been discussed already long ago, e.g., in Ref. [16]. The cleanest possibility is obviously the addition of a second observable which exactly mirrors the behaviour of $F_{2}$, i.e., is directly sensitive only to the gluon distribution at LO. An example already mentioned in Ref. [16] is deepinelastic scattering through the exchange of a scalar $\phi$ interacting with hadrons only via a $\phi G^{\mu v} G_{\mu v}$ coupling to the gluon field-strength tensor. This interaction is realized in the Standard Model by the Higgs boson, for a very heavy top quark and negligible couplings to all other flavours [22].

In fact, as discussed in Section 2, the corresponding coefficient functions had to be calculated to order $\alpha_{\mathrm{s}}^{2}$ in the approach of Refs. [23] to the determination of the complete set of third-order (NNLO) splitting functions, carried out for all- $N /$ all- $x$ in Ref. [7]. The numerous three-loop integrals for the latter were evaluated to order $\varepsilon^{0}$ in dimensional regularization in order to obtain also the third-order coefficient functions for $F_{2}, F_{L}$ and $F_{3}$ [8-10]. Hence also the three-loop coefficient functions for the $\phi$-exchange structure function $F_{\phi}$ could be calculated with a relatively minor extra effort. In Section 3 of this article we have presented and discussed these coefficient functions.

While, of course, Higgs-exchange DIS is of no experimental relevance at any past or foreseeable lepton-nucleon facility, these results have already proven useful in theoretical research as discussed above. Both the quark and gluon coefficient functions for $F_{\phi}$ show, as expected, considerably larger higher-order corrections than their counterparts for $F_{2}$. Their most prominent feature is the double-logarithmic enhancement of the $1 / x$-contributions dominating their small- $x$ behaviour, i.e., the occurrence of terms up to $x^{-1} \ln ^{2 n} x$ in the $n$-loop coefficient functions $c_{\phi, i}^{(n)}(x)$. These terms arise from using the large- $m_{\text {top }}$ effective interaction outside its region of validity, see also Ref. [53] for a recent discussion in the context of Higgs production in proton collisions.

The above double-logarithmic contributions also render the physical evolution kernels for the system $\left(F_{2}, F_{\phi}\right)$, presented and discussed in Section 4 , unstable within the physically interesting region of $x>10^{-4}$ relevant to crucial high-scale processes at the LHC. Consequently this scheme is unattractive for practical analyses of hard processes. There are various possibilities, theoretical and phenomenological, to improve upon this situation which we, however, leave to future research. Already the present heavy-top results for the coefficient functions, however, can provide useful checks of, e.g., the order-dependence of fitted $\overline{\mathrm{MS}}$ gluon densities by comparing the resulting shapes of the (in principle order-independent) quantity $F_{\phi}$ outside the small- $x$ region.

The above flavour-singlet evolution-kernel matrices share a theoretically very interesting feature with the (scalar) physical kernels for a rather wide range of non-singlet observables [19, 20]: They show a single-logarithmic enhancement at large values of the scaling variable $x$, i.e., terms of the form $(1-x)^{a} \ln ^{k}(1-x)$ with $k>n$ do not contribute to the $\mathrm{N}^{n} \mathrm{LO}$ physical kernels for any value of $a$. This structure is in contrast to that of all coefficient functions and (pure-) singlet splitting functions. We consider this feature, which is already established to $\mathrm{N}^{3} \mathrm{LO}$ for non-singlet structure functions, as a general property of the physical evolution kernels, and consequently conjecture that it holds to $\mathrm{N}^{3} \mathrm{LO}$ also for the present $\left(F_{2}, F_{\phi}\right)$ singlet case. 
Hence the double-logarithmic contributions from (a) the four-loop splitting functions and (b) the scheme transformation including lower orders and the three-loop coefficient functions have to cancel, i.e., the so far unknown corresponding terms in (a) can be inferred from our now complete results for (b). We have provided and discussed these predictions in Section 5. In particular, we note that the known double logarithms in the splitting functions to NNLO exhibit a particular colour structure in powers of $\left(C_{A}-C_{F}\right)$ which is non-trivially fulfilled also by our $\mathrm{N}^{3} \mathrm{LO}$ predictions, a fact that we consider a significant check of their correctness.

As also discussed in Section 5, the predicted contributions to the splitting functions $P_{i k}^{(3)}(x)$ alone are clearly insufficient for use in phenomenological applications. However they will become very useful in connection with future fourth-order calculations, such as the extension of Ref. [28] to higher moments and the flavour-singlet sector. Already the corresponding three-loop predictions, had they been known at the time, would have led to more accurate $x$-space approximation on the basis of the moments calculated in Refs. [23] than those actually obtained in Refs. [44,65]. In fact, this situation clearly illustrates the general importance of calculations to at least NNLO: It usually requires two non-trivial orders to reliably identify most general structures - for another example recall the 'curious incident' of the coefficient of $\ln (1-x)$ in the three-loop diagonal splitting functions [6,7] which triggered Ref. [15] which in turn greatly assisted further developments including the determination of the NNLO splitting functions for the final-state fragmentation case $[29,66]$. We hope, accordingly, that also our present results will assist further developments in perturbative QCD, e.g., in the approach introduced in Ref. [60].

FORM files of our main formulae, and FORTRAN subroutines of the $\phi$-exchange coefficient functions and the resulting physical evolution kernels can be obtained from the preprint server HTTP://arXiv.org by downloading the source of this article. Furthermore they are available from the authors upon request.

\section{Acknowledgments}

The work of G.S. has been funded by the UK Science \& Technology Facilities Council (STFC). S.M. acknowledges support by the Helmholtz Gemeinschaft under contract VH-NG-105 and in part by the Deutsche Forschungsgemeinschaft in Sonderforschungsbereich/Transregio 9. The work of J.V. has been part of the research program of the Dutch Foundation for Fundamental Research of Matter (FOM). The research of A.V., who thanks S. Marzani for a useful discussion, has been supported by STFC under grant numbers PP/E007414/1 and ST/G00062X/1. 


\section{Appendix A: The third-order coefficient functions for $F_{\phi}$}

In this appendix we present the very lengthy exact expressions for the third-order scalar-exchange coefficient functions $c_{\phi, \mathrm{q}}^{(3)}(x)$ and $c_{\phi, \mathrm{g}}^{(3)}(x)$. The notation is the same as used for the first- and secondorder results given in Eqs. (3.5) - (3.9) above. The result for the quark coefficient function reads

$$
\begin{aligned}
& c_{\phi, \mathrm{q}}^{(3)}(x)=C_{F} C_{A}^{2}\left(p _ { \mathrm { gq } } ( x ) \left(-1652 / 15 \mathrm{H}_{1} \zeta_{2}^{2}+240 \mathrm{H}_{1,-3,0}-160 \mathrm{H}_{1,-2} \zeta_{2}+256 \mathrm{H}_{1,-2,0,0}\right.\right. \\
& -704 / 3 \mathrm{H}_{1,-2,-1,0}+128 / 3 \mathrm{H}_{1,-2,2}+712 / 3 \mathrm{H}_{1,0} \zeta_{3}+680 / 3 \mathrm{H}_{1,0,0} \zeta_{2}+32 \mathrm{H}_{1,1,-2,0} \\
& -376 / 3 \mathrm{H}_{1,0,0,0,0}+80 / 3 \mathrm{H}_{1,1} \zeta_{3}+168 \mathrm{H}_{1,1,0} \zeta_{2}-248 / 3 \mathrm{H}_{1,1,0,0,0}+736 / 3 \mathrm{H}_{1,1,1} \zeta_{2} \\
& -896 / 3 \mathrm{H}_{1,1,1,0,0}-1208 / 3 \mathrm{H}_{1,1,1,1,0}-400 \mathrm{H}_{1,1,1,1,1}-1208 / 3 \mathrm{H}_{1,1,1,2}-392 \mathrm{H}_{1,1,2,0} \\
& -1232 / 3 \mathrm{H}_{1,1,2,1}-288 \mathrm{H}_{1,1,3}+776 / 3 \mathrm{H}_{1,2} \zeta_{2}-784 / 3 \mathrm{H}_{1,2,0,0}-384 \mathrm{H}_{1,2,1,0} \\
& \left.-1216 / 3 \mathrm{H}_{1,2,1,1}-1192 / 3 \mathrm{H}_{1,2,2}-1024 / 3 \mathrm{H}_{1,3,0}-1144 / 3 \mathrm{H}_{1,3,1}-272 \mathrm{H}_{1,4}\right) \\
& +p_{\mathrm{gq}}(-x)\left(-988 / 15 \mathrm{H}_{-1} \zeta_{2}^{2}-992 / 3 \mathrm{H}_{-1,-3,0}+1712 / 3 \mathrm{H}_{-1,-2} \zeta_{2}-432 \mathrm{H}_{-1,-2,2}\right. \\
& +832 / 3 \mathrm{H}_{-1,-2,-1,0}-1688 / 3 \mathrm{H}_{-1,-2,0,0}+2240 / 3 \mathrm{H}_{-1,-1} \zeta_{3}+272 \mathrm{H}_{-1,-1,-2,0} \\
& -2120 / 3 \mathrm{H}_{-1,-1,-1} \zeta_{2}-944 / 3 \mathrm{H}_{-1,-1,-1,-1,0}+2128 / 3 \mathrm{H}_{-1,-1,-1,0,0} \\
& +1648 / 3 \mathrm{H}_{-1,-1,-1,2}+2464 / 3 \mathrm{H}_{-1,-1,0} \zeta_{2}-1832 / 3 \mathrm{H}_{-1,-1,0,0,0}-272 \mathrm{H}_{-1,-1,2,0} \\
& -992 / 3 \mathrm{H}_{-1,-1,2,1}-2104 / 3 \mathrm{H}_{-1,-1,3}-536 \mathrm{H}_{-1,0} \zeta_{3}-1160 / 3 \mathrm{H}_{-1,0,0} \zeta_{2} \\
& +232 \mathrm{H}_{-1,0,0,0,0}+64 / 3 \mathrm{H}_{-1,2} \zeta_{2}+304 / 3 \mathrm{H}_{-1,2,0,0}+224 / 3 \mathrm{H}_{-1,2,1,0}+96 \mathrm{H}_{-1,2,2} \\
& \left.+256 / 3 \mathrm{H}_{-1,2,1,1}+632 / 3 \mathrm{H}_{-1,3,0}+784 / 3 \mathrm{H}_{-1,3,1}+1024 / 3 \mathrm{H}_{-1,4}\right) \\
& -35864 / 27\left(1-8 / 4483 / x^{2}+46675 / 53796 / x+7755 / 17932 x\right. \\
& \left.+3883 / 13449 x^{2}\right) \mathrm{H}_{-1,0}+32 / 3(1-75 / x-56 x) \mathrm{H}_{2,1,1,1}+80 / 3(1-162 / 5 / x \\
& -267 / 10 x) \mathrm{H}_{-2,0,0,0}+208 / 3(1-48 / 13 / x-279 / 26 x) \mathrm{H}_{5}+112 / 3(1-24 / 7 / x \\
& -29 / 14 x) \mathrm{H}_{-3,2}-208 / 3(1-36 / 13 / x-127 / 13 x) \mathrm{H}_{0,0,0} \zeta_{2} \\
& +2368 / 15(1-349 / 148 / x-96 / 37 x) \mathrm{H}_{0} \zeta_{2}^{2}-6112 / 9(1-841 / 382 / x+85 / 764 x \\
& \left.+54 / 191 x^{2}\right) \mathrm{H}_{2} \zeta_{2}-2620 / 9\left(1-1356 / 655 / x+187 / 262 x+44 / 655 x^{2}\right) \mathrm{H}_{1} \zeta_{3} \\
& +10040 / 81\left(1-16057 / 10040 / x+24191 / 10040 x-865 / 251 x^{2}\right) \mathrm{H}_{1,1} \\
& -72928 / 27\left(1-55601 / 36464 / x-9461 / 36464 x-1697 / 9116 x^{2}\right) \zeta_{3} \\
& +59026 / 81\left(1-86857 / 59026 / x+24793 / 59026 x-17892 / 29513 x^{2}\right) \mathrm{H}_{1,0} \\
& +7976 / 9\left(1-1312 / 997 / x-229 / 1994 x+60 / 997 x^{2}\right) \mathrm{H}_{1,0,0,0} \\
& -9376 / 9\left(1-382 / 293 / x+55 / 586 x+32 / 293 x^{2}\right) \mathrm{H}_{1,1} \zeta_{2} \\
& -2648 / 3\left(1-1289 / 993 / x-1 / 331 x+80 / 993 x^{2}\right) \mathrm{H}_{1,0} \zeta_{2} \\
& +11420 / 9\left(1-3704 / 2855 / x+257 / 2855 x+336 / 2855 x^{2}\right) \mathrm{H}_{1,1,0,0} \\
& +12092 / 9\left(1-3614 / 3023 / x+107 / 3023 x+312 / 3023 x^{2}\right) \mathrm{H}_{1,1,2} \\
& +11200 / 9\left(1-208 / 175 / x-73 / 1400 x+3 / 35 x^{2}\right) \mathrm{H}_{1,3}+11240 / 9(1-1664 / 1405 / x
\end{aligned}
$$


$\left.+161 / 2810 x+148 / 1405 x^{2}\right) \mathrm{H}_{1,1,1,1}+12076 / 9(1-3562 / 3019 / x+103 / 3019 x$

$\left.+312 / 3019 x^{2}\right) \mathrm{H}_{1,2,1}+1336\left(1-195 / 167 / x+6 / 167 x+52 / 501 x^{2}\right) \mathrm{H}_{1,1,1,0}$

$+13252 / 9\left(1-3852 / 3313 / x-53 / 3313 x+320 / 3313 x^{2}\right) \mathrm{H}_{1,2,0}$

$+1289237 / 243(1-1478082 / 1289237 / x-205504 / 1289237 x$

$\left.+167284 / 1289237 x^{2}\right) \mathrm{H}_{1}-68764 / 27(1-38843 / 34382 / x-4969 / 34382 x$

$\left.+432 / 17191 x^{2}\right) \mathrm{H}_{1} \zeta_{2}+1328 / 9\left(1-91 / 83 / x-89 / 166 x+8 / 83 x^{2}\right) \mathrm{H}_{1,-2,0}$

$+72760 / 27\left(1-19543 / 18190 / x-419 / 1819 x+9 / 9095 x^{2}\right) \mathrm{H}_{1,0,0}$

$+11480 / 9\left(1-1527 / 1435 / x+421 / 2870 x+268 / 1435 x^{2}\right) \mathrm{H}_{2,0,0}$

$+54620 / 27\left(1-27851 / 27310 / x-2458 / 13655 x-146 / 2731 x^{2}\right) \mathrm{H}_{1,2}$

$+48764 / 27\left(1-24351 / 24382 / x-4361 / 24382 x-782 / 12191 x^{2}\right) \mathrm{H}_{1,1,1}$

$+56864 / 27\left(1-28103 / 28432 / x-365 / 1777 x-383 / 7108 x^{2}\right) \mathrm{H}_{1,1,0}$

$+9412 / 9\left(1-2040 / 2353 / x+1046 / 2353 x+800 / 2353 x^{2}\right) \mathrm{H}_{4}$

$+13468 / 9\left(1-2866 / 3367 / x+557 / 3367 x+648 / 3367 x^{2}\right) \mathrm{H}_{2,2}$

$+13736 / 9\left(1-1431 / 1717 / x+623 / 3434 x+320 / 1717 x^{2}\right) \mathrm{H}_{2,1,1}$

$+4904 / 3\left(1-507 / 613 / x+247 / 1839 x+316 / 1839 x^{2}\right) \mathrm{H}_{2,1,0}$

$-9412 / 9\left(1-1932 / 2353 / x+956 / 2353 x+800 / 2353 x^{2}\right) \mathrm{H}_{0,0} \zeta_{2}$

$+5200 / 3(1-523 / 650 / x-597 / 1300 x) \zeta_{5}+12472 / 9(1-1128 / 1559 / x$

$\left.+511 / 1559 x+368 / 1559 x^{2}\right) \mathrm{H}_{3,0}+15968 / 9(1-1095 / 1996 / x+1303 / 3992 x$

$\left.+102 / 499 x^{2}\right) \mathrm{H}_{3,1}+85748 / 27(1-10598 / 21437 / x-3388 / 21437 x$

$\left.+70 / 1261 x^{2}\right) \mathrm{H}_{2,0}-3896\left(1-1888 / 4383 / x+200 / 4383 x+580 / 4383 x^{2}\right) \mathrm{H}_{0} \zeta_{3}$

$+122288 / 27\left(1-3219 / 7643 / x-6949 / 61144 x+1455 / 15286 x^{2}\right) \mathrm{H}_{3}$

$-122288 / 27\left(1-12395 / 30572 / x-3535 / 61144 x+1455 / 15286 x^{2}\right) \mathrm{H}_{0} \zeta_{2}$

$-160(1-2 / 5 / x-1 / 10 x) \mathrm{H}_{2,-2,0}+27832 / 9(1-8245 / 20874 / x-739 / 6958 x$

$\left.+391 / 10437 x^{2}\right) \mathrm{H}_{2,1}+1600 / 3(1-9 / 25 / x-61 / 200 x) \mathrm{H}_{-3,0,0}$

$+3047527 / 243(1-1062210 / 3047527 / x-139147 / 6095054 x$

$\left.+522652 / 3047527 x^{2}\right) \mathrm{H}_{0}+170344 / 27(1-13635 / 42586 / x-4134 / 21293 x$

$\left.+3017 / 21293 x^{2}\right) \mathrm{H}_{0,0,0}+4904 / 3\left(1-166 / 613 / x-129 / 613 x+72 / 613 x^{2}\right) \mathrm{H}_{-2,-1,0}$

$+7796 / 3\left(1-270 / 1949 / x+121 / 1949 x+216 / 1949 x^{2}\right) \mathrm{H}_{-2} \zeta_{2}+480(1-2 / 15 / x$

$-11 / 10 x) \mathrm{H}_{0,0,0,0,0}-31780 / 9\left(1-206 / 1589 / x-54 / 1135 x+928 / 7945 x^{2}\right) \mathrm{H}_{-2,0,0}$

$-381824 / 81\left(1-180471 / 1527296 / x-2124809 / 4581888 x+250877 / 572736 x^{2}\right)$

$+2384 / 3(1-12 / 149 / x-25 / 298 x) \mathrm{H}_{-4,0}-5344 / 3(1-13 / 167 / x+125 / 668 x$

$\left.+18 / 167 x^{2}\right) \mathrm{H}_{-2,2}+74912 / 27(1-481 / 18728 / x-1707 / 18728 x$

$\left.+1305 / 4682 x^{2}\right) \mathrm{H}_{-2,0}-25544 / 9\left(1+54 / 3193 / x-45 / 3193 x+400 / 3193 x^{2}\right) \mathrm{H}_{-3,0}$ 
$+832(1+1 / 6 / x+69 / 104 x) \mathrm{H}_{0,0} \zeta_{3}+9464 / 9(1+512 / 1183 / x+509 / 1183 x$ $\left.+40 / 1183 x^{2}\right) \mathrm{H}_{-1,-1,2}-4060 / 3\left(1+226 / 435 / x+13 / 35 x+136 / 3045 x^{2}\right) \mathrm{H}_{-1,-1} \zeta_{2}$

$-1112 / 3(1+76 / 139 / x+347 / 278 x) \zeta_{2} \zeta_{3}+2800 / 3(1+4 / 7 / x+117 / 350 x) \mathrm{H}_{-2,-1,2}$ $+4732 / 3\left(1+748 / 1183 / x+789 / 2366 x+76 / 1183 x^{2}\right) \mathrm{H}_{-1} \zeta_{3}$

$-3488 / 3(1+72 / 109 / x+85 / 218 x) \mathrm{H}_{-2,-1} \zeta_{2}-7528 / 9(1+648 / 941 / x$

$\left.-167 / 1882 x-268 / 941 x^{2}\right) \mathrm{H}_{0,0,0,0}-16780 / 9(1+3232 / 4195 / x$

$\left.+1274 / 4195 x+336 / 4195 x^{2}\right) \mathrm{H}_{-1,3}+4648 / 3(1+65 / 83 / x+92 / 581 x$

$\left.+8 / 83 x^{2}\right) \mathrm{H}_{-1,-1,0,0}+3176 / 3(1+314 / 397 / x+389 / 794 x) \mathrm{H}_{-2} \zeta_{3}$

$-7984 / 9\left(1+396 / 499 / x+319 / 998 x+44 / 499 x^{2}\right) \mathrm{H}_{-1,2,1}$

$+20036 / 9\left(1+3982 / 5009 / x+1414 / 5009 x+416 / 5009 x^{2}\right) \mathrm{H}_{-1,0} \zeta_{2}$

$+186364 / 81\left(1+37486 / 46591 / x-6167 / 186364 x+22118 / 46591 x^{2}\right) \zeta_{2}$

$-5432 / 9\left(1+558 / 679 / x+113 / 679 x+8 / 97 x^{2}\right) \mathrm{H}_{-1,-1,-1,0}$

$-7240 / 9\left(1+752 / 905 / x+283 / 905 x+72 / 905 x^{2}\right) \mathrm{H}_{-1,2,0}$

$+576\left(1+71 / 81 / x+17 / 216 x+8 / 81 x^{2}\right) \mathrm{H}_{-1,-2,0}+144(1+8 / 9 / x$

$+37 / 54 x) \mathrm{H}_{-3,-1,0}-19328 / 9\left(1+601 / 604 / x+751 / 4832 x+63 / 604 x^{2}\right) \mathrm{H}_{-1,0,0,0}$

$-424(1+160 / 159 / x+427 / 318 x) \mathrm{H}_{3,0,0}-1376 / 3(1+44 / 43 / x$

$+53 / 86 x) \mathrm{H}_{-2,-1,-1,0}-293866 / 81(1+151137 / 146933 / x+8738 / 146933 x$

$\left.+73960 / 146933 x^{2}\right) \mathrm{H}_{0,0}+59324 / 27(1+15328 / 14831 / x+2398 / 14831 x$

$\left.+4676 / 14831 x^{2}\right) \mathrm{H}_{-1,0,0}+24536 / 27(1+3303 / 3067 / x+385 / 6134 x$

$\left.+1178 / 3067 x^{2}\right) \mathrm{H}_{-1,2}-704 / 3(1+25 / 22 / x+43 / 44 x) \mathrm{H}_{2,0,0,0}$

$-896 / 3(1+8 / 7 / x+11 / 14 x) \mathrm{H}_{-2,2,1}-2024 / 3(1+292 / 253 / x+37 / 46 x) \mathrm{H}_{-2,3}$

$-38680 / 27\left(1+6051 / 4835 / x+823 / 19340 x+1759 / 4835 x^{2}\right) \mathrm{H}_{-1} \zeta_{2}$

$-656 / 3(1+52 / 41 / x+75 / 82 x) \mathrm{H}_{-2,2,0}-186364 / 81(1+121647 / 93182 / x$

$\left.+40363 / 186364 x+22118 / 46591 x^{2}\right) \mathrm{H}_{2}+2144 / 3(1+88 / 67 / x+125 / 134 x) \mathrm{H}_{-2,0} \zeta_{2}$

$-1336 / 3(1+224 / 167 / x+415 / 334 x) \mathrm{H}_{2,1,0,0}+240(1+8 / 5 / x+11 / 10 x) \mathrm{H}_{-2,-2,0}$

$+1912 / 3(1+348 / 239 / x+485 / 478 x) \mathrm{H}_{-2,-1,0,0}+408(1+224 / 153 / x$

$+509 / 306 x) \mathrm{H}_{3} \zeta_{2}-28288 / 27\left(1+687 / 442 / x+53 / 7072 x+581 / 1768 x^{2}\right) \mathrm{H}_{-1,-1,0}$

$+512 / 3(1+53 / 32 / x+93 / 64 x) \mathrm{H}_{2} \zeta_{3}-1168 / 3(1+124 / 73 / x+271 / 146 x) \mathrm{H}_{3,1,0}$

$+880 / 3(1+102 / 55 / x+171 / 110 x) \mathrm{H}_{2,1} \zeta_{2}-336(1+124 / 63 / x+13 / 6 x) \mathrm{H}_{3,2}$

$-632 / 3(1+168 / 79 / x+547 / 158 x) \mathrm{H}_{4,0}-896 / 3(1+18 / 7 / x+37 / 14 x) \mathrm{H}_{3,1,1}$

$-616 / 3(1+204 / 77 / x+629 / 154 x) \mathrm{H}_{4,1}+368 / 3(1+82 / 23 / x+119 / 46 x) \mathrm{H}_{2,0} \zeta_{2}$

$-488 / 3(1+260 / 61 / x+421 / 122 x) \mathrm{H}_{2,2,0}+104 / 3(1+72 / 13 / x+95 / 26 x) \mathrm{H}_{-3} \zeta_{2}$

$-9986 / 45\left(1+27874 / 4993 / x+1529 / 4993 x-328 / 4993 x^{2}\right) \zeta_{2}^{2}$ 
$-248 / 3(1+224 / 31 / x+331 / 62 x) \mathrm{H}_{2,3}-64(1+73 / 6 / x+28 / 3 x) \mathrm{H}_{2,1,2}$

$\left.-160 / 3(1+73 / 5 / x+56 / 5 x) \mathrm{H}_{2,2,1}-128 / 3(1+73 / 4 / x+14 x) \mathrm{H}_{2,1,1,0}\right)$

$+C_{F}^{2} C_{A}\left(p_{\mathrm{gq}}(x)\left(-1856 / 15 \mathrm{H}_{1} \zeta_{2}^{2}-176 \mathrm{H}_{1,-3,0}+264 \mathrm{H}_{1,-2} \zeta_{2}+112 \mathrm{H}_{1,-2,-1,0}\right.\right.$

$-640 / 3 \mathrm{H}_{1,-2,0,0}-208 \mathrm{H}_{1,-2,2}-32 \mathrm{H}_{1,0} \zeta_{3}+640 / 3 \mathrm{H}_{1,0,0} \zeta_{2}-544 / 3 \mathrm{H}_{1,0,0,0,0}$

$+632 / 3 \mathrm{H}_{1,1} \zeta_{3}-464 / 3 \mathrm{H}_{1,1,-2,0}+1400 / 3 \mathrm{H}_{1,1,0} \zeta_{2}-928 / 3 \mathrm{H}_{1,1,0,0,0}$

$+784 / 3 \mathrm{H}_{1,1,1} \zeta_{2}-320 / 3 \mathrm{H}_{1,1,1,0,0}-40 \mathrm{H}_{1,1,1,1,0}-104 \mathrm{H}_{1,1,1,2}-120 \mathrm{H}_{1,1,2,0}$

$-304 / 3 \mathrm{H}_{1,1,2,1}-432 \mathrm{H}_{1,1,3}+832 / 3 \mathrm{H}_{1,2} \zeta_{2}-592 / 3 \mathrm{H}_{1,2,0,0}-368 / 3 \mathrm{H}_{1,2,1,0}$

$\left.-80 \mathrm{H}_{1,2,1,1}-488 / 3 \mathrm{H}_{1,2,2}-176 \mathrm{H}_{1,3,0}-440 / 3 \mathrm{H}_{1,3,1}-616 / 3 \mathrm{H}_{1,4}\right)$

$+p_{\mathrm{gq}}(-x)\left(1016 / 15 \mathrm{H}_{-1} \zeta_{2}^{2}+192 \mathrm{H}_{-1,-3,0}-344 / 3 \mathrm{H}_{-1,-2} \zeta_{2}-688 / 3 \mathrm{H}_{-1,-2,-1,0}\right.$

$+488 / 3 \mathrm{H}_{-1,-2,0,0}-136 \mathrm{H}_{-1,-1} \zeta_{3}-224 \mathrm{H}_{-1,-1,-2,0}+296 / 3 \mathrm{H}_{-1,-1,-1} \zeta_{2}$

$+944 / 3 \mathrm{H}_{-1,-1,-1,-1,0}-736 / 3 \mathrm{H}_{-1,-1,-1,0,0}+176 / 3 \mathrm{H}_{-1,-1,-1,2}$

$-112 / 3 \mathrm{H}_{-1,-1,0} \zeta_{2}+80 \mathrm{H}_{-1,-1,0,0,0}+112 / 3 \mathrm{H}_{-1,-1,2,0}+160 / 3 \mathrm{H}_{-1,-1,2,1}$

$+8 / 3 \mathrm{H}_{-1,-1,3}-448 / 3 \mathrm{H}_{-1,0} \zeta_{3}-320 / 3 \mathrm{H}_{-1,0,0} \zeta_{2}+96 \mathrm{H}_{-1,0,0,0,0}-232 / 3 \mathrm{H}_{-1,2} \zeta_{2}$

$+368 / 3 \mathrm{H}_{-1,2,0,0}+128 / 3 \mathrm{H}_{-1,2,1,0}+32 / 3 \mathrm{H}_{-1,2,1,1}+64 / 3 \mathrm{H}_{-1,2,2}+184 / 3 \mathrm{H}_{-1,3,0}$

$\left.+64 \mathrm{H}_{-1,3,1}+344 / 3 \mathrm{H}_{-1,4}\right)+1280\left(1-7 / 1080 / x^{2}+139 / 640 / x+15683 / 17280 x\right.$

$\left.-7 / 240 x^{2}\right) \mathrm{H}_{-1,0}+742 / 27\left(1-13896 / 371 / x+59 / 2 x-144 / 53 x^{2}\right) \zeta_{3}$

$+904 / 9\left(1-543 / 113 / x+853 / 226 x-64 / 113 x^{2}\right) \mathrm{H}_{0} \zeta_{3}-128 / 3(1-15 / 8 / x$

$-1 / 4 x) \mathrm{H}_{-2,0,0,0}-27938 / 27(1-22578 / 13969 / x-35941 / 27938 x$

$\left.-720 / 13969 x^{2}\right) \mathrm{H}_{0} \zeta_{2}+27938 / 27(1-21120 / 13969 / x-27781 / 27938 x$

$\left.-720 / 13969 x^{2}\right) \mathrm{H}_{3}+5032 / 9\left(1-936 / 629 / x-1715 / 1258 x+12 / 629 x^{2}\right) \mathrm{H}_{2,1,1}$

$+176(1-16 / 11 / x-107 / 66 x) \mathrm{H}_{3,1,0}+1120 / 3(1-46 / 35 / x-31 / 35 x) \mathrm{H}_{2,1,0,0}$

$+34460 / 27\left(1-10674 / 8615 / x-1888 / 8615 x-72 / 8615 x^{2}\right) \mathrm{H}_{2,0}$

$+784 / 3(1-8 / 7 / x-13 / 14 x) \mathrm{H}_{2,2,0}+41896 / 27(1-5901 / 5237 / x$

$\left.-4243 / 20948 x+288 / 5237 x^{2}\right) \mathrm{H}_{2,1}+688 / 3(1-48 / 43 / x-125 / 86 x) \mathrm{H}_{3,2}$

$+43340 / 27\left(1-4745 / 4334 / x-3007 / 10835 x+24 / 2167 x^{2}\right) \mathrm{H}_{1,2}$

$+38416 / 27\left(1-20597 / 19208 / x-961 / 4802 x+30 / 2401 x^{2}\right) \mathrm{H}_{1,1,0}$

$+43012 / 27\left(1-23041 / 21506 / x-2357 / 10753 x+544 / 10753 x^{2}\right) \mathrm{H}_{1,1,1}$

$+6128 / 9\left(1-807 / 766 / x-605 / 766 x+30 / 383 x^{2}\right) \mathrm{H}_{2,1,0}$

$+78766 / 27\left(1-82071 / 78766 / x-21843 / 78766 x+20 / 39383 x^{2}\right) \mathrm{H}_{1,0}$

$+7304 / 9\left(1-927 / 913 / x-1483 / 1826 x+60 / 913 x^{2}\right) \mathrm{H}_{2,2}+736 / 3(1-1 / x$

$-35 / 46 x) \mathrm{H}_{2,1,1,0}+8728 / 9\left(1-1073 / 1091 / x-436 / 1091 x+24 / 1091 x^{2}\right) \mathrm{H}_{1,2,0}$

$+274384 / 81\left(1-33685 / 34298 / x-20807 / 68596 x-331 / 34298 x^{2}\right) \mathrm{H}_{1,1}$

$+10688 / 9\left(1-653 / 668 / x-503 / 1336 x+3 / 167 x^{2}\right) \mathrm{H}_{1,1,0,0}+768(1-23 / 24 / x$ 
$\left.-143 / 288 x+1 / 72 x^{2}\right) \mathrm{H}_{1,1,1,0}+1923029 / 486(1-1747009 / 1923029 / x$ $\left.-795761 / 3846058 x-40576 / 1923029 x^{2}\right) \mathrm{H}_{1}+9224 / 9(1-1003 / 1153 / x$ $\left.-1105 / 2306 x+12 / 1153 x^{2}\right) \mathrm{H}_{1,2,1}-286819 / 81(1-19056 / 22063 / x$ $\left.-181409 / 286819 x+360 / 286819 x^{2}\right) \zeta_{2}+1262 / 27(1-540 / 631 / x$ $\left.-17713 / 1262 x-1600 / 631 x^{2}\right) \mathrm{H}_{0,0,0}+7528 / 9(1-796 / 941 / x-583 / 941 x$ $\left.-4 / 941 x^{2}\right) \mathrm{H}_{1,1,1,1}+8552 / 9\left(1-898 / 1069 / x-1039 / 2138 x+12 / 1069 x^{2}\right) \mathrm{H}_{1,1,2}$ $+8792 / 9\left(1-894 / 1099 / x-1945 / 2198 x+132 / 1099 x^{2}\right) \mathrm{H}_{3,1}$ $+848 / 3(1-42 / 53 / x-97 / 106 x) \mathrm{H}_{3,1,1}+286819 / 81(1-225210 / 286819 / x$ $\left.-87311 / 286819 x+360 / 286819 x^{2}\right) \mathrm{H}_{2}-52040 / 27(1-4073 / 5204 / x$ $\left.-4477 / 13010 x+36 / 6505 x^{2}\right) \mathrm{H}_{1} \zeta_{2}-480(1-7 / 9 / x-31 / 60 x) \mathrm{H}_{2} \zeta_{3}$ $-5800 / 9\left(1-112 / 145 / x+98 / 145 x-16 / 725 x^{2}\right) \mathrm{H}_{-1,-1,0}$ $+1023301 / 486(1-789822 / 1023301 / x-203321 / 1023301 x$ $\left.-6016 / 1023301 x^{2}\right) \mathrm{H}_{0}-13160 / 9(1-181 / 235 / x-1627 / 3290 x$ $\left.+24 / 1645 x^{2}\right) \mathrm{H}_{1,1} \zeta_{2}+13972 / 9(1-2686 / 3493 / x-1709 / 6986 x$ $\left.-32 / 3493 x^{2}\right) \mathrm{H}_{1,0,0}+1088 / 3(1-13 / 17 / x-43 / 68 x) \mathrm{H}_{2,2,1}$ $+1216 / 3(1-29 / 38 / x-11 / 19 x) \mathrm{H}_{2,1,2}-1156(1-1916 / 2601 / x-281 / 578 x$ $\left.+80 / 2601 x^{2}\right) \mathrm{H}_{1} \zeta_{3}-2368 / 3(1-47 / 74 / x-65 / 148 x) \mathrm{H}_{2,1} \zeta_{2}$ $+3008 / 3\left(1-467 / 752 / x-293 / 752 x+1 / 47 x^{2}\right) \mathrm{H}_{1,0,0,0}$ $+12904 / 9\left(1-995 / 1613 / x-1553 / 3226 x+24 / 1613 x^{2}\right) \mathrm{H}_{1,3}$ $+4472 / 9\left(1-1719 / 2795 / x-4153 / 11180 x+348 / 2795 x^{2}\right) \zeta_{2}^{2}$ $-10856 / 9\left(1-825 / 1357 / x-1729 / 2714 x+96 / 1357 x^{2}\right) \mathrm{H}_{2} \zeta_{2}$ $+640 / 3(1-3 / 5 / x-2 / 5 x) \mathrm{H}_{-2,-1,2}+1472 / 3(1-55 / 92 / x-13 / 23 x) \mathrm{H}_{2,0,0,0}$ $-13864 / 9\left(1-1028 / 1733 / x-1667 / 3466 x+36 / 1733 x^{2}\right) \mathrm{H}_{1,0} \zeta_{2}$ $+10120 / 9\left(1-732 / 1265 / x-1469 / 2530 x+144 / 1265 x^{2}\right) \mathrm{H}_{3,0}$ $-528(1-6 / 11 / x-68 / 99 x) \mathrm{H}_{3} \zeta_{2}+304(1-10 / 19 / x-17 / 38 x) \mathrm{H}_{2,1,1,1}$ $+1088 / 3(1-71 / 136 / x-1 / 2 x) \mathrm{H}_{1,-2,0}+8968 / 9(1-567 / 1121 / x-989 / 2242 x$ $\left.+72 / 1121 x^{2}\right) \mathrm{H}_{2,0,0}+1360 / 3(1-42 / 85 / x-117 / 170 x) \mathrm{H}_{3,0,0}$ $+2315033 / 324\left(1-140715 / 330719 / x-306555 / 2315033 x-7104 / 2315033 x^{2}\right)$ $-2560 / 3(1-17 / 40 / x-31 / 80 x) \mathrm{H}_{2,0} \zeta_{2}+2432 / 3(1-31 / 76 / x-29 / 76 x) \mathrm{H}_{2,3}$ $-14632 / 9\left(1-24 / 59 / x-2159 / 3658 x+240 / 1829 x^{2}\right) \mathrm{H}_{0,0} \zeta_{2}$ $+1184 / 3(1-15 / 37 / x-19 / 74 x) \mathrm{H}_{2,-2,0}+1472 / 3(1-37 / 92 / x-75 / 184 x) \zeta_{2} \zeta_{3}$ $+14632 / 9\left(1-690 / 1829 / x-1919 / 3658 x+240 / 1829 x^{2}\right) \mathrm{H}_{4}$ $+800 / 3(1-9 / 25 / x-4 / 25 x) \mathrm{H}_{-2,0} \zeta_{2}+127235 / 81(1-44982 / 127235 / x$ 
$\left.-301739 / 254470 x-3352 / 127235 x^{2}\right) \mathrm{H}_{0,0}+304 / 3(1-6 / 19 / x+26 / 19 x) \mathrm{H}_{0,0} \zeta_{3}$ $-8084 / 9\left(1-514 / 2021 / x+1900 / 2021 x-48 / 2021 x^{2}\right) \mathrm{H}_{-1} \zeta_{2}$

$-2368 / 3\left(1-17 / 74 / x+41 / 148 x+3 / 37 x^{2}\right) \mathrm{H}_{-2,-1,0}+2848 / 15(1-39 / 178 / x$ $-407 / 712 x) \mathrm{H}_{0} \zeta_{2}^{2}-928 / 3(1-6 / 29 / x-2 / 29 x) \mathrm{H}_{-2,3}+7556 / 9(1-318 / 1889 / x$ $\left.+1750 / 1889 x-32 / 1889 x^{2}\right) \mathrm{H}_{-1,0,0}-1120(1-4 / 105 / x-11 / 420 x$ $\left.+3 / 35 x^{2}\right) \mathrm{H}_{-2} \zeta_{2}+2704 / 3\left(1-7 / 338 / x+12 / 169 x+20 / 169 x^{2}\right) \mathrm{H}_{-2,0,0}$ $+576\left(1+23 / 648 / x+235 / 216 x-2 / 81 x^{2}\right) \mathrm{H}_{-1,2}+2176 / 3(1+9 / 136 / x$ $\left.-13 / 68 x+3 / 34 x^{2}\right) \mathrm{H}_{-2,2}+464(1+2 / 29 / x+13 / 174 x) \mathrm{H}_{-3,0,0}$ $-1312 / 3(1+3 / 41 / x+19 / 164 x) \mathrm{H}_{-3} \zeta_{2}+1904 / 3(1+9 / 119 / x+20 / 119 x$ $\left.+12 / 119 x^{2}\right) \mathrm{H}_{-3,0}-1792 / 3(1+3 / 28 / x+11 / 112 x) \mathrm{H}_{-3,-1,0}$ $-1120 / 3(1+6 / 35 / x+33 / 35 x) \mathrm{H}_{5}+1120 / 3(1+6 / 35 / x+34 / 35 x) \mathrm{H}_{0,0,0} \zeta_{2}$ $+4576 / 9\left(1+243 / 1144 / x+85 / 143 x-4 / 143 x^{2}\right) \mathrm{H}_{-2,0}+16(1+1 / 3 / x-13 / 6 x$ $\left.+4 / 3 x^{2}\right) \mathrm{H}_{-1,2,1}-824\left(1+109 / 309 / x+235 / 618 x+8 / 103 x^{2}\right) \mathrm{H}_{-1,-1,0,0}$ $-576\left(1+31 / 72 / x+53 / 108 x+1 / 27 x^{2}\right) \mathrm{H}_{-1,-2,0}+1024 / 3(1+111 / 256 / x$ $\left.+97 / 256 x+3 / 16 x^{2}\right) \mathrm{H}_{-1,0,0,0}-480(1+7 / 15 / x+3 / 10 x) \mathrm{H}_{-2,-2,0}-64 \mathrm{H}_{-2,2,1}$ $-432\left(1+38 / 81 / x+31 / 162 x+4 / 81 x^{2}\right) \mathrm{H}_{-1,-1,2}-312(1+56 / 117 / x+1 / 6 x) \zeta_{5}$ $+768(1+1 / 2 / x+7 / 24 x) \mathrm{H}_{-2,-1,-1,0}+944(1+199 / 354 / x+43 / 118 x$ $\left.+2 / 59 x^{2}\right) \mathrm{H}_{-1,-1} \zeta_{2}-836\left(1+122 / 209 / x+149 / 418 x+32 / 627 x^{2}\right) \mathrm{H}_{-1} \zeta_{3}$ $-464\left(1+18 / 29 / x+37 / 174 x+14 / 87 x^{2}\right) \mathrm{H}_{-1,0} \zeta_{2}-240(1+83 / 90 x) \mathrm{H}_{0,0,0,0,0}$ $+1024\left(1+41 / 64 / x+49 / 96 x+1 / 48 x^{2}\right) \mathrm{H}_{-1,-1,-1,0}+1072 / 3(1+97 / 134 / x$ $\left.+9 / 67 x+12 / 67 x^{2}\right) \mathrm{H}_{-1,3}+272 / 3\left(1+13 / 17 / x+2 / 17 x+4 / 17 x^{2}\right) \mathrm{H}_{-1,2,0}$ $-1024 / 3(1+15 / 16 / x+35 / 64 x) \mathrm{H}_{-2,-1,0,0}-320 / 3(1+6 / 5 / x+59 / 20 x) \mathrm{H}_{4,0}$ $-160(1+9 / 5 / x+21 / 20 x) \mathrm{H}_{-2} \zeta_{3}+512 / 3(1+15 / 8 / x+37 / 32 x) \mathrm{H}_{-2,-1} \zeta_{2}$ $-64(1+3 / x+67 / 12 x) \mathrm{H}_{4,1}+13952 / 9\left(1-337 / 872 x+13 / 109 x^{2}\right) \mathrm{H}_{0,0,0,0}$ $\left.-160 / 3(1-1 / 10 x) \mathrm{H}_{-2,2,0}+1952 / 3(1+1 / 61 x) \mathrm{H}_{-4,0}+416 / 3(1+2 / 13 x) \mathrm{H}_{-3,2}\right)$ $+C_{F}^{3}\left(p_{\mathrm{gq}}(x)\left(1288 / 15 \mathrm{H}_{1} \zeta_{2}^{2}+640 / 3 \mathrm{H}_{1,-3,0}-304 \mathrm{H}_{1,-2} \zeta_{2}-160 \mathrm{H}_{1,-2,-1,0}\right.\right.$ $+256 \mathrm{H}_{1,-2,0,0}+224 \mathrm{H}_{1,-2,2}+400 / 3 \mathrm{H}_{1,0} \zeta_{3}-96 \mathrm{H}_{1,0,0} \zeta_{2}+64 \mathrm{H}_{1,0,0,0,0}$ $-352 / 3 \mathrm{H}_{1,1} \zeta_{3}+416 / 3 \mathrm{H}_{1,1,-2,0}-944 / 3 \mathrm{H}_{1,1,0} \zeta_{2}+96 \mathrm{H}_{1,1,0,0,0}-368 / 3 \mathrm{H}_{1,1,1} \zeta_{2}$ $-608 / 3 \mathrm{H}_{1,1,1,0,0}-400 / 3 \mathrm{H}_{1,1,1,1,0}-80 \mathrm{H}_{1,1,1,1,1}-208 / 3 \mathrm{H}_{1,1,1,2}-128 \mathrm{H}_{1,1,2,0}$ $-64 \mathrm{H}_{1,1,2,1}+208 \mathrm{H}_{1,1,3}-96 \mathrm{H}_{1,2} \zeta_{2}-272 / 3 \mathrm{H}_{1,2,0,0}-400 / 3 \mathrm{H}_{1,2,1,0}$ $\left.-272 / 3 \mathrm{H}_{1,2,1,1}-80 \mathrm{H}_{1,2,2}-64 \mathrm{H}_{1,3,0}-80 \mathrm{H}_{1,3,1}+64 \mathrm{H}_{1,4}\right)$ $+p_{\mathrm{gq}}(-x)\left(736 / 15 \mathrm{H}_{-1} \zeta_{2}^{2}-832 / 3 \mathrm{H}_{-1,-3,0}+400 \mathrm{H}_{-1,-2} \zeta_{2}+352 \mathrm{H}_{-1,-2,-1,0}\right.$ $-1504 / 3 \mathrm{H}_{-1,-2,0,0}-224 \mathrm{H}_{-1,-2,2}+464 \mathrm{H}_{-1,-1} \zeta_{3}+352 \mathrm{H}_{-1,-1,-2,0}$ 
$-448 \mathrm{H}_{-1,-1,-1} \zeta_{2}-384 \mathrm{H}_{-1,-1,-1,-1,0}+704 \mathrm{H}_{-1,-1,-1,0,0}+256 \mathrm{H}_{-1,-1,-1,2}$

$+1472 / 3 \mathrm{H}_{-1,-1,0} \zeta_{2}-1552 / 3 \mathrm{H}_{-1,-1,0,0,0}-128 \mathrm{H}_{-1,-1,2,0}-128 \mathrm{H}_{-1,-1,2,1}$

$-384 \mathrm{H}_{-1,-1,3}-112 \mathrm{H}_{-1,0} \zeta_{3}-240 \mathrm{H}_{-1,0,0} \zeta_{2}+448 / 3 \mathrm{H}_{-1,0,0,0,0}+80 \mathrm{H}_{-1,2} \zeta_{2}$

$\left.-96 \mathrm{H}_{-1,2,0,0}+64 \mathrm{H}_{-1,3,0}+64 \mathrm{H}_{-1,3,1}+208 \mathrm{H}_{-1,4}\right)-6752 / 3\left(1-2 / 633 / x^{2}\right.$

$+183 / 844 / x+4349 / 5064 x) \mathrm{H}_{-1,0}-88 / 3(1-210 / 11 / x-21 / 2 x) \zeta_{5}$

$+424 / 15(1-519 / 53 / x+4 / 53 x) \zeta_{2}^{2}+284 / 3(1-581 / 284 / x+887 / 284 x) \mathrm{H}_{1,1}$

$+1088 / 3(1-171 / 136 / x-65 / 136 x) \mathrm{H}_{1,2,1}+920 / 3(1-57 / 46 / x-67 / 115 x) \mathrm{H}_{2,1}$

$+976 / 3(1-75 / 61 / x-101 / 244 x) \mathrm{H}_{1,1,1,1}+1216 / 3(1-93 / 76 / x$

$-71 / 152 x) \mathrm{H}_{1,1,2}+1340 / 3(1-358 / 335 / x-88 / 335 x) \mathrm{H}_{1,1,1}$

$+1856 / 3(1-30 / 29 / x-27 / 58 x) \mathrm{H}_{1,1,1,0}+1136 / 3(1-72 / 71 / x-44 / 71 x) \mathrm{H}_{2,2}$

$+1436 / 3(1-364 / 359 / x-23 / 718 x) \mathrm{H}_{1,2}+2036 / 3(1-504 / 509 / x$

$-193 / 1018 x) \mathrm{H}_{1,1,0}+1424 / 3(1-84 / 89 / x-83 / 178 x) \mathrm{H}_{1,2,0}$

$+1600 / 3(1-9 / 10 / x-131 / 200 x) \mathrm{H}_{2,1,0}-1664 / 3(1-93 / 104 / x$

$-25 / 52 x) \mathrm{H}_{1,-2,0}+1472 / 3(1-81 / 92 / x-187 / 368 x) \mathrm{H}_{2,1,1}$

$+916 / 3(1-180 / 229 / x-167 / 458 x) \mathrm{H}_{2,0}-3265 / 6(1-493 / 653 / x$

$-4527 / 6530 x) \mathrm{H}_{1}+896 / 3(1-3 / 4 / x-1 / 2 x) \mathrm{H}_{2,2,1}+784 / 3(1-36 / 49 / x$

$-1 / 2 x) \mathrm{H}_{2,1,1,1}+800 / 3(1-18 / 25 / x-27 / 50 x) \mathrm{H}_{2,1,2}+1232 / 3(1-54 / 77 / x$

$-34 / 77 x) \mathrm{H}_{3,1}+1216 / 3(1-12 / 19 / x-9 / 19 x) \mathrm{H}_{2,1,1,0}+240(1-3 / 5 / x$

$-3 / 5 x) \mathrm{H}_{3,0}+1024 / 3(1-9 / 16 / x-15 / 32 x) \mathrm{H}_{2,2,0}-70927 / 36(1-2098 / 3733 / x$

$-11754 / 70927 x)+1088 / 3(1-9 / 17 / x-1 / 2 x) \mathrm{H}_{3,2}-1088 / 3(1-9 / 17 / x$

$-11 / 34 x) \mathrm{H}_{2,-2,0}+368(1-12 / 23 / x-1 / 2 x) \mathrm{H}_{3,1,1}+424(1-55 / 106 / x$

$+91 / 212 x) \mathrm{H}_{1,0}+1312 / 3(1-18 / 41 / x-1 / 2 x) \mathrm{H}_{3,1,0}+1232 / 3(1-24 / 77 / x$

$-13 / 22 x) \mathrm{H}_{1,1} \zeta_{2}+448(1-2 / 7 / x-3 / 7 x) \mathrm{H}_{4,1}-596(1-36 / 149 / x$

$+271 / 447 x) \mathrm{H}_{0} \zeta_{3}+512 / 3(1-15 / 64 / x-77 / 128 x) \mathrm{H}_{1,1,0,0}+832 / 3(1-3 / 13 / x$

$-3 / 26 x) \mathrm{H}_{2,1} \zeta_{2}-1000 / 3(1-27 / 125 / x-189 / 250 x) \mathrm{H}_{1,0,0}+568(1-15 / 71 / x$

$-121 / 213 x) \mathrm{H}_{1} \zeta_{3}+336(1-4 / 21 / x-17 / 42 x) \mathrm{H}_{4,0}+880 / 3(1-3 / 22 / x$

$+689 / 220 x) \mathrm{H}_{3}-1592 / 3(1-24 / 199 / x-55 / 398 x) \mathrm{H}_{0,0} \zeta_{3}+976 / 3(1-6 / 61 / x$

$-33 / 122 x) \mathrm{H}_{2,1,0,0}+2060 / 3(1-32 / 515 / x-861 / 1030 x) \mathrm{H}_{1} \zeta_{2}-288(1-1 / 18 / x$

$-19 / 36 x) \mathrm{H}_{1,0,0,0}+4352 / 3(1+3 / 68 / x+15 / 272 x) \mathrm{H}_{-3} \zeta_{2}-4192 / 3(1+6 / 131 / x$

$+21 / 262 x) \mathrm{H}_{-3,0,0}-1304(1+19 / 326 / x+1607 / 978 x) \zeta_{3}-3584 / 3(1+9 / 112 / x$

$+33 / 224 x) \mathrm{H}_{-3,0}-1056(1+1 / 11 / x+5 / 33 x) \mathrm{H}_{-2,2}+3712 / 3(1+3 / 29 / x$

$+15 / 116 x) \mathrm{H}_{-3,-1,0}-7120 / 3(1+99 / 890 / x+121 / 445 x) \mathrm{H}_{-2,0}+845(1+8 / 65 / x$

$+39127 / 15210 x) \mathrm{H}_{0,0}+1424 / 3(1+12 / 89 / x-9 / 178 x) \mathrm{H}_{2,0} \zeta_{2}$ 
$+5248 / 3(1+27 / 164 / x+167 / 656 x) \mathrm{H}_{-2} \zeta_{2}-736(1+4 / 23 / x+9 / 46 x) \mathrm{H}_{-2,3}$ $-2720 / 3(1+3 / 17 / x+41 / 170 x) \mathrm{H}_{-2,0,0,0}+176(1+2 / 11 / x-5 / 22 x) \mathrm{H}_{2} \zeta_{3}$ $-5392 / 3(1+63 / 337 / x+217 / 674 x) \mathrm{H}_{-2,0,0}+960(1+1 / 5 / x+2 / 9 x) \mathrm{H}_{-2,0} \zeta_{2}$ $+1648 / 3(1+24 / 103 / x-273 / 412 x) \mathrm{H}_{1,0} \zeta_{2}-1264(1+19 / 79 / x+62 / 79 x) \mathrm{H}_{-1,2}$ $+4160 / 3(1+18 / 65 / x+107 / 260 x) \mathrm{H}_{-2,-1,0}+168(1+1 / 3 / x+1079 / 504 x) \mathrm{H}_{2}$ $+4576 / 3(1+48 / 143 / x+81 / 286 x) \mathrm{H}_{-2,-1,0,0}+7288 / 3(1+312 / 911 / x$ $+593 / 911 x) \mathrm{H}_{-1} \zeta_{2}-2528(1+85 / 237 / x+151 / 237 x) \mathrm{H}_{-1,0,0}+1152(1+7 / 18 / x$ $+7 / 24 x) \mathrm{H}_{-2} \zeta_{3}+2432 / 3(1+15 / 38 / x+6 / 19 x) \mathrm{H}_{-2,-2,0}+640(1+2 / 5 / x$ $+3 / 10 x) \mathrm{H}_{-2,-1,2}-224(1+3 / 7 / x+4 / 7 x)\left(\mathrm{H}_{-1,2,0}+\mathrm{H}_{-1,2,1}\right)$ $-1184(1+16 / 37 / x+23 / 74 x) \mathrm{H}_{-2,-1} \zeta_{2}+6992 / 3(1+198 / 437 / x$ $+221 / 437 x) \mathrm{H}_{-1,-1,0}-1088(1+8 / 17 / x+11 / 34 x) \mathrm{H}_{-2,-1,-1,0}$ $-1088(1+65 / 136 / x+55 / 102 x) \mathrm{H}_{-1,0,0,0}+3472 / 3(1+108 / 217 / x$ $+249 / 434 x) \mathrm{H}_{-1,0} \zeta_{2}+256(1+1 / 2 / x+19 / 48 x) \mathrm{H}_{3} \zeta_{2}-864(1+1 / 2 / x$ $+7 / 12 x) \mathrm{H}_{-1,3}-752 / 3(1+24 / 47 / x+17 / 94 x) \mathrm{H}_{2,3}+368 / 3(1+12 / 23 / x$ $-5 / 46 x) \zeta_{2} \zeta_{3}+944 / 3(1+36 / 59 / x-19 / 118 x) \mathrm{H}_{2} \zeta_{2}+5984 / 3(1+21 / 34 / x$ $+395 / 748 x) \mathrm{H}_{-1,-1,0,0}+928(1+18 / 29 / x+31 / 58 x) \mathrm{H}_{-1,-1,2}$ $+1640(1+138 / 205 / x+221 / 410 x) \mathrm{H}_{-1} \zeta_{3}+3424 / 3(1+147 / 214 / x$ $+55 / 107 x) \mathrm{H}_{-1,-2,0}-1744(1+75 / 109 / x+58 / 109 x) \mathrm{H}_{-1,-1} \zeta_{2}-832 \mathrm{H}_{-3,2}$ $-880 / 3(1+42 / 55 / x+1173 / 220 x) \mathrm{H}_{0} \zeta_{2}-1632(1+13 / 17 / x$ $+9 / 17 x) \mathrm{H}_{-1,-1,-1,0}-256(1+17 / 16 / x-51 / 64 x) \mathrm{H}_{1,3}-236 / 3(1+72 / 59 / x$ $+365 / 59 x) \mathrm{H}_{0,0} \zeta_{2}-208 / 3(1+18 / 13 / x+105 / 52 x) \mathrm{H}_{2,0,0}-272 / 5(1+40 / 17 / x$ $+2 x) \mathrm{H}_{0} \zeta_{2}^{2}-168(1+68 / 21 / x+20633 / 1512 x) \zeta_{2}+2437 / 18(1+7972 / 2437 / x$ $+5058 / 2437 x) \mathrm{H}_{0}-160 / 3(1-147 / 20 x) \mathrm{H}_{0,0,0,0}+328(1-21 / 82 x) \mathrm{H}_{5}$ $-416 / 3(1-5 / 26 x) \mathrm{H}_{2,0,0,0}-328(1-13 / 82 x) \mathrm{H}_{0,0,0} \zeta_{2}-2816 / 3(1+3 / 88 x) \mathrm{H}_{-4,0}$ $-192(1+1 / 6 x)\left(\mathrm{H}_{-2,2,0}+\mathrm{H}_{-2,2,1}\right)+120(1+43 / 90 x) \mathrm{H}_{0,0,0,0,0}$ $\left.+1172 / 3(1+950 / 293 x) \mathrm{H}_{0,0,0}+236 / 3(1+233 / 59 x) \mathrm{H}_{4}-64(1 / x+3 / 2 x) \mathrm{H}_{3,0,0}\right)$ $+C_{F} C_{A} n_{f}\left(p_{\mathrm{gq}}(x)\left(100 / 9 \mathrm{H}_{1} \zeta_{3}+160 / 9 \mathrm{H}_{1,-2,0}-184 / 3 \mathrm{H}_{1,0} \zeta_{2}+688 / 9 \mathrm{H}_{1,0,0,0}\right.\right.$ $-560 / 9 \mathrm{H}_{1,1} \zeta_{2}+544 / 9 \mathrm{H}_{1,1,0,0}+128 / 3 \mathrm{H}_{1,1,1,0}+376 / 9 \mathrm{H}_{1,1,1,1}+448 / 9 \mathrm{H}_{1,1,2}$ $\left.+512 / 9 \mathrm{H}_{1,2,0}+416 / 9 \mathrm{H}_{1,2,1}+560 / 9 \mathrm{H}_{1,3}\right)+p_{\mathrm{gq}}(-x)\left(-20 \mathrm{H}_{-1} \zeta_{3}-16 \mathrm{H}_{-1,-2,0}\right.$ $+80 / 3 \mathrm{H}_{-1,-1} \zeta_{2}+224 / 9 \mathrm{H}_{-1,-1,-1,0}-32 \mathrm{H}_{-1,-1,0,0}-128 / 9 \mathrm{H}_{-1,-1,2}$ $\left.+112 / 9 \mathrm{H}_{-1,0} \zeta_{2}-256 / 9 \mathrm{H}_{-1,0,0,0}-128 / 9 \mathrm{H}_{-1,2,0}-80 / 9 \mathrm{H}_{-1,2,1}-104 / 9 \mathrm{H}_{-1,3}\right)$ $-5744 / 27\left(1+2 / 359 / x^{2}+1751 / 4308 / x+1049 / 1436 x+113 / 1077 x^{2}\right) \mathrm{H}_{-1,0}$ $-64 / 9(1-29 / 2 / x-23 / 2 x) \mathrm{H}_{2,2}-176 / 9(1-63 / 11 / x-103 / 22 x) \mathrm{H}_{3,1}$ 
$-176 / 9(1-5 / x-7 / 2 x) \mathrm{H}_{2,1,1}-15527 / 27(1-398483 / 139743 / x$

$\left.-17114 / 139743 x-512 / 46581 x^{2}\right)-128 / 3(1-11 / 4 / x-5 / 3 x) \mathrm{H}_{2,1,0}$

$+448 / 9(1-5 / 2 / x-7 / 4 x) \mathrm{H}_{2} \zeta_{2}-512 / 9(1-5 / 2 / x-53 / 32 x) \mathrm{H}_{2,0,0}$

$-202498 / 243\left(1-183399 / 101249 / x-84161 / 202498 x-17064 / 101249 x^{2}\right) \mathrm{H}_{0}$

$+80 / 3(1-5 / 3 / x+7 / 15 x) \mathrm{H}_{0} \zeta_{3}+7376 / 27(1-1325 / 922 / x-1517 / 1844 x$

$\left.+68 / 461 x^{2}\right) \mathrm{H}_{0} \zeta_{2}-7376 / 27\left(1-627 / 461 / x-1271 / 1844 x+68 / 461 x^{2}\right) \mathrm{H}_{3}$

$-110708 / 243\left(1-133181 / 110708 / x-26707 / 110708 x-6468 / 27677 x^{2}\right) \mathrm{H}_{1}$

$+11128 / 27\left(1-3301 / 2782 / x-1556 / 1391 x-10 / 1391 x^{2}\right) \zeta_{3}$

$-12256 / 27\left(1-1761 / 1532 / x-2001 / 3064 x+59 / 383 x^{2}\right) \mathrm{H}_{0,0,0}$

$-2560 / 9\left(1-533 / 480 / x-2 / 5 x+7 / 80 x^{2}\right) \mathrm{H}_{2,1}-45940 / 81(1-24377 / 22970 / x$

$\left.-5353 / 22970 x-36 / 11485 x^{2}\right) \mathrm{H}_{1,0}-9152 / 27(1-2407 / 2288 / x-667 / 2288 x$

$\left.+1 / 22 x^{2}\right) \mathrm{H}_{1,2}-9728 / 27\left(1-1261 / 1216 / x-467 / 1216 x+29 / 304 x^{2}\right) \mathrm{H}_{2,0}$

$-34412 / 81\left(1-1273 / 1229 / x-1474 / 8603 x+164 / 8603 x^{2}\right) \mathrm{H}_{1,1}$

$-86756 / 81\left(1-22113 / 21689 / x-7885 / 21689 x+1064 / 21689 x^{2}\right) \mathrm{H}_{0,0}$

$-13120 / 27\left(1-833 / 820 / x-577 / 1640 x+7 / 205 x^{2}\right) \mathrm{H}_{1,0,0}$

$+10624 / 27\left(1-84 / 83 / x-899 / 2656 x+29 / 664 x^{2}\right) \mathrm{H}_{1} \zeta_{2}$

$-8264 / 27\left(1-2085 / 2066 / x-643 / 2066 x+44 / 1033 x^{2}\right) \mathrm{H}_{1,1,1}-64 / 3(1-1 / x$

$-x) \mathrm{H}_{-2,2}-2704 / 45(1-168 / 169 / x-185 / 338 x) \zeta_{2}^{2}-9776 / 27(1-2391 / 2444 / x$

$\left.-823 / 2444 x+22 / 611 x^{2}\right) \mathrm{H}_{1,1,0}+59024 / 81(1-3207 / 3689 / x-8753 / 29512 x$

$\left.+303 / 3689 x^{2}\right) \zeta_{2}-59024 / 81(1-11077 / 14756 / x-2459 / 29512 x$

$\left.+303 / 3689 x^{2}\right) \mathrm{H}_{2}-640 / 9(1-3 / 10 / x-3 / 10 x) \mathrm{H}_{-3,0}-320 / 9(1-1 / 5 / x$

$-3 / 5 x) \mathrm{H}_{-2,0,0}+1312 / 27\left(1-1 / 8 / x+83 / 82 x+9 / 82 x^{2}\right) \mathrm{H}_{-1} \zeta_{2}$

$-1664 / 27\left(1-7 / 208 / x+79 / 104 x+1 / 4 x^{2}\right) \mathrm{H}_{-1,0,0}-3392 / 27(1+71 / 424 / x$

$\left.+123 / 424 x+13 / 106 x^{2}\right) \mathrm{H}_{-2,0}+256 / 3(1+1 / 2 / x+1 / 8 x) \mathrm{H}_{-2,-1,0}$

$+1072 / 9(1+42 / 67 / x+143 / 134 x) \mathrm{H}_{0,0,0,0}+2944 / 27(1+281 / 368 / x+29 / 46 x$

$\left.+3 / 92 x^{2}\right) \mathrm{H}_{-1,-1,0}+160 / 27\left(1+161 / 20 / x-5 / 2 x-3 / 5 x^{2}\right) \mathrm{H}_{-1,2}$

$-80 / 9(1+54 / 5 / x+113 / 10 x) \mathrm{H}_{0,0} \zeta_{2}+80 / 9(1+66 / 5 / x+137 / 10 x) \mathrm{H}_{4}$

$\left.+32 / 9(1+33 / x+55 / 2 x) \mathrm{H}_{3,0}+64(1-1 / 4 x) \mathrm{H}_{-2} \zeta_{2}\right)$

$+C_{F}^{2} n_{f}\left(p_{\mathrm{gq}}(x)\left(-128 / 3 \mathrm{H}_{1} \zeta_{3}-64 / 3 \mathrm{H}_{1,-2,0}-8 / 9 \mathrm{H}_{1,0} \zeta_{2}-16 / 3 \mathrm{H}_{1,0,0,0}\right.\right.$

$+104 / 9 \mathrm{H}_{1,1} \zeta_{2}+88 / 9 \mathrm{H}_{1,1,0,0}+56 / 3 \mathrm{H}_{1,1,1,0}+104 / 9 \mathrm{H}_{1,1,1,1}+88 / 9 \mathrm{H}_{1,1,2}$

$\left.+272 / 9 \mathrm{H}_{1,2,0}+184 / 9 \mathrm{H}_{1,2,1}+104 / 9 \mathrm{H}_{1,3}\right)+p_{\mathrm{gq}}(-x)\left(112 / 3 \mathrm{H}_{-1} \zeta_{3}\right.$

$+128 / 3 \mathrm{H}_{-1,-2,0}-128 / 3 \mathrm{H}_{-1,-1} \zeta_{2}-128 / 3 \mathrm{H}_{-1,-1,-1,0}+224 / 3 \mathrm{H}_{-1,-1,0,0}$

$\left.+64 / 3 \mathrm{H}_{-1,-1,2}+64 / 3 \mathrm{H}_{-1,0} \zeta_{2}-32 \mathrm{H}_{-1,0,0,0}-32 / 3 \mathrm{H}_{-1,3}\right)$ 
$+(2-x)\left(-56 / 5 \mathrm{H}_{0} \zeta_{2}^{2}+152 / 3 \mathrm{H}_{0,0} \zeta_{3}+88 \mathrm{H}_{0,0,0} \zeta_{2}-120 \mathrm{H}_{0,0,0,0,0}+16 \mathrm{H}_{3} \zeta_{2}\right.$

$\left.-16 \mathrm{H}_{3,0,0}-16 \mathrm{H}_{3,1,0}-16 \mathrm{H}_{3,1,1}-16 \mathrm{H}_{3,2}-48 \mathrm{H}_{4,0}-48 \mathrm{H}_{4,1}-88 \mathrm{H}_{5}\right)$

$+(2-x)\left(24 \zeta_{5}-32 \zeta_{2} \zeta_{3}\right)-112 / 9(1-25 / 7 / x-2 / 7 x) \mathrm{H}_{2,0,0}+64 / 3(1-31 / 12 / x$

$\left.+13 / 4 x-1 / 3 x^{2}\right) \mathrm{H}_{-1,2}+28030 / 81(1-16948 / 14015 / x+2234 / 14015 x$

$\left.-1488 / 14015 x^{2}\right) \zeta_{2}-544 / 9(1-39 / 34 / x-7 / 68 x) \mathrm{H}_{2,1,1}$

$-28030 / 81\left(1-3168 / 2803 / x-9646 / 14015 x-1488 / 14015 x^{2}\right) \mathrm{H}_{2}$

$-608 / 9(1-21 / 19 / x-11 / 76 x) \mathrm{H}_{2,1,0}-704 / 9(1-12 / 11 / x-17 / 88 x) \mathrm{H}_{2,2}$

$+1072 / 9(1-72 / 67 / x-53 / 268 x) \mathrm{H}_{0,0} \zeta_{2}-848 / 9(1-54 / 53 / x+19 / 106 x) \mathrm{H}_{3,1}$

$-880 / 9(1-54 / 55 / x+17 / 110 x) \mathrm{H}_{3,0}+4640 / 27(1-142 / 145 / x-101 / 232 x$

$\left.+9 / 145 x^{2}\right) \mathrm{H}_{1} \zeta_{2}-1072 / 9(1-60 / 67 / x+19 / 268 x) \mathrm{H}_{4}-7712 / 27(1-823 / 964 / x$

$\left.-1087 / 1928 x+6 / 241 x^{2}\right) \mathrm{H}_{1,2}-1624 / 9(1-139 / 174 / x-95 / 203 x$

$\left.+32 / 609 x^{2}\right) \mathrm{H}_{1,0,0}-6448 / 27\left(1-1279 / 1612 / x-445 / 806 x+12 / 403 x^{2}\right) \mathrm{H}_{1,1,1}$

$-7552 / 27\left(1-185 / 236 / x-1007 / 1888 x+3 / 118 x^{2}\right) \mathrm{H}_{1,1,0}$

$-9148 / 27\left(1-1790 / 2287 / x-1323 / 2287 x-192 / 2287 x^{2}\right) \mathrm{H}_{1,0}$

$-246517 / 243\left(1-185803 / 246517 / x-181615 / 493034 x-5280 / 246517 x^{2}\right) \mathrm{H}_{1}$

$-28300 / 81\left(1-5272 / 7075 / x-907 / 1415 x-576 / 7075 x^{2}\right) \mathrm{H}_{1,1}$

$+2528 / 9(1-43 / 79 / x-247 / 632 x) \mathrm{H}_{0} \zeta_{3}-19352 / 81(1-2481 / 4838 / x$

$\left.+19385 / 19352 x-392 / 2419 x^{2}\right) \mathrm{H}_{0,0}-585697 / 486(1-842416 / 1757091 / x$

$\left.-129025 / 585697 x-9872 / 251013 x^{2}\right)-12872 / 27(1-705 / 1609 / x$

$\left.-1111 / 3218 x+72 / 1609 x^{2}\right) \mathrm{H}_{2,0}-12832 / 27(1-351 / 802 / x-139 / 401 x$

$\left.+18 / 401 x^{2}\right) \mathrm{H}_{2,1}-440 / 9(1-24 / 55 / x+89 / 110 x) \mathrm{H}_{0,0,0,0}$

$-254765 / 243\left(1-14591 / 36395 / x-103486 / 254765 x-2592 / 254765 x^{2}\right) \mathrm{H}_{0}$

$+18752 / 27\left(1-439 / 1172 / x-839 / 9376 x+12 / 293 x^{2}\right) \mathrm{H}_{0} \zeta_{2}$

$-18752 / 27\left(1-189 / 586 / x-1343 / 9376 x+12 / 293 x^{2}\right) \mathrm{H}_{3}$

$+12176 / 27\left(1-239 / 761 / x+3553 / 6088 x+14 / 761 x^{2}\right) \zeta_{3}$

$+320 / 3(1-1 / 5 / x-3 / 10 x) \mathrm{H}_{-2,2}+2528 / 9(1-61 / 474 / x+21 / 158 x$

$\left.-10 / 237 x^{2}\right) \mathrm{H}_{-2,0}+832 / 3\left(1-277 / 2808 / x+55 / 52 x+5 / 702 x^{2}\right) \mathrm{H}_{-1,0}$

$+320(1-1 / 15 / x-1 / 10 x) \mathrm{H}_{-3,0}-18896 / 27(1-15 / 1181 / x-151 / 4724 x$

$\left.+52 / 1181 x^{2}\right) \mathrm{H}_{0,0,0}+832 / 3(1+1 / 39 / x-3 / 26 x) \mathrm{H}_{-2,0,0}-608 / 3(1+1 / 19 / x$

$-5 / 38 x) \mathrm{H}_{-2} \zeta_{2}+1504 / 9\left(1+19 / 282 / x+55 / 47 x-10 / 141 x^{2}\right) \mathrm{H}_{-1,0,0}$

$-1216 / 9\left(1+23 / 152 / x+175 / 152 x-3 / 38 x^{2}\right) \mathrm{H}_{-1} \zeta_{2}-192(1+1 / 3 / x$

$+1 / 18 x) \mathrm{H}_{-2,-1,0}+5096 / 45(1+246 / 637 / x-1 / 14 x) \zeta_{2}^{2}-2048 / 9(1+85 / 128 / x$

$\left.\left.+97 / 128 x-1 / 32 x^{2}\right) \mathrm{H}_{-1,-1,0}-160 / 9(1+3 / x+11 / 20 x) \mathrm{H}_{2} \zeta_{2}\right)$ 


$$
\begin{aligned}
+ & C_{F} n_{f}^{2}\left(p _ { \mathrm { gq } } ( x ) \left(8 / 3 \zeta_{3}+64 / 9 \mathrm{H}_{0} \zeta_{2}-16 \mathrm{H}_{0,0,0}+40 / 9 \mathrm{H}_{1} \zeta_{2}-64 / 9 \mathrm{H}_{1,0,0}\right.\right. \\
& \left.-40 / 9 \mathrm{H}_{1,1,0}-32 / 9 \mathrm{H}_{1,1,1}-40 / 9 \mathrm{H}_{1,2}-64 / 9 \mathrm{H}_{2,0}-40 / 9 \mathrm{H}_{2,1}-64 / 9 \mathrm{H}_{3}\right) \\
& +13726 / 243(1-25291 / 13726 / x-5807 / 13726 x)+5440 / 81(1-959 / 680 / x \\
& -59 / 136 x) \mathrm{H}_{0}+1712 / 27(1-241 / 214 / x-101 / 214 x) \mathrm{H}_{0,0} \\
& +1036 / 27(1-551 / 518 / x-239 / 518 x) \mathrm{H}_{1}-848 / 27(1-1 / x-25 / 53 x)\left(\zeta_{2}-\mathrm{H}_{1,0}\right. \\
& \left.\left.-\mathrm{H}_{2}\right)+536 / 27(1-125 / 134 / x-61 / 134 x) \mathrm{H}_{1,1}\right) .
\end{aligned}
$$

The corresponding expression for the gluon coefficient function is given by

$$
\begin{aligned}
& c_{\phi, \mathrm{g}}^{(3)}(x)=C_{A}^{3}\left(-44228 / 27\left(1-8 / 11057 / x^{2}+8977 / 11057 / x+11031 / 11057 x\right.\right. \\
& \left.+8959 / 11057 x^{2}\right) \mathrm{H}_{-1,0}-7262 / 27\left(1-19412 / 3631 / x-842 / 3631 x+16705 / 3631 x^{2}\right) \zeta_{3} \\
& +12724 / 27\left(1-48356 / 9543 / x-16641 / 6362 x+67957 / 19086 x^{2}\right) \mathrm{H}_{1,1} \\
& -608 / 3\left(1-275 / 57 / x-153 / 76 x+869 / 228 x^{2}\right) \mathrm{H}_{1,0} \zeta_{2}+7672 / 9(1-59035 / 17262 / x \\
& \left.-739 / 411 x+12371 / 4932 x^{2}\right) \mathrm{H}_{1,0}+544\left(1-77 / 34 / x-113 / 34 x+66 / 17 x^{2}\right) \mathrm{H}_{2,1,1} \\
& +408\left(1-110 / 51 / x-383 / 306 x+583 / 306 x^{2}\right) \mathrm{H}_{1,0,0,0}+5968 / 9(1-759 / 373 / x \\
& \left.-1955 / 1492 x+2563 / 1492 x^{2}\right) \mathrm{H}_{1,3}-6200 / 9(1-1573 / 775 / x-941 / 775 x \\
& \left.+1397 / 775 x^{2}\right) \mathrm{H}_{1,1} \zeta_{2}-4616 / 3(1-20489 / 10386 / x-1483 / 1154 x \\
& \left.+18311 / 10386 x^{2}\right) \mathrm{H}_{1} \zeta_{2}+8128 / 9\left(1-913 / 508 / x-623 / 508 x+803 / 508 x^{2}\right) \mathrm{H}_{1,1,1,0} \\
& +13448 / 9\left(1-8963 / 5043 / x-4355 / 3362 x+7874 / 5043 x^{2}\right) \mathrm{H}_{1,2} \\
& +6544 / 9\left(1-726 / 409 / x-4373 / 1636 x+5357 / 1636 x^{2}\right) \mathrm{H}_{2,2}+800(1-44 / 25 / x \\
& \left.-61 / 50 x+77 / 50 x^{2}\right) \mathrm{H}_{1,1,1,1}+8536 / 9(1-169 / 97 / x-2597 / 2134 x \\
& \left.+295 / 194 x^{2}\right)\left(\mathrm{H}_{1,1,2}+\mathrm{H}_{1,2,1}\right)+13960 / 9(1-2943 / 1745 / x-871 / 698 x \\
& \left.+5039 / 3490 x^{2}\right) \mathrm{H}_{1,1,1}+9832 / 9\left(1-2013 / 1229 / x-1426 / 1229 x+1826 / 1229 x^{2}\right) \mathrm{H}_{1,2,0} \\
& +137744 / 27\left(1-13910 / 8609 / x-492137 / 413232 x+568297 / 413232 x^{2}\right) \mathrm{H}_{1} \\
& +15400 / 9\left(1-9326 / 5775 / x-4593 / 3850 x+7874 / 5775 x^{2}\right) \mathrm{H}_{1,1,0} \\
& +10904 / 9\left(1-2189 / 1363 / x-1529 / 1363 x+2013 / 1363 x^{2}\right) \mathrm{H}_{1,1,0,0} \\
& +16040 / 9\left(1-3041 / 2005 / x-4621 / 4010 x+5477 / 4010 x^{2}\right) \mathrm{H}_{1,0,0} \\
& +7304 / 9\left(1-123 / 83 / x-1916 / 913 x+248 / 83 x^{2}\right) \mathrm{H}_{2,1,0}+7544 / 9(1-33 / 23 / x \\
& \left.-1931 / 943 x+2541 / 943 x^{2}\right) \mathrm{H}_{2,0,0}+7784 / 9(1-880 / 973 / x-4183 / 1946 x \\
& \left.+1067 / 278 x^{2}\right) \mathrm{H}_{3,1}+2668 / 3\left(1-583 / 667 / x-3995 / 2001 x+7139 / 2001 x^{2}\right) \mathrm{H}_{3,0} \\
& +68572 / 27\left(1-13324 / 17143 / x-599 / 553 x+28492 / 17143 x^{2}\right) \mathrm{H}_{2,0} \\
& +62572 / 27\left(1-10990 / 15643 / x-14213 / 15643 x+27766 / 15643 x^{2}\right) \mathrm{H}_{2,1} \\
& +788\left(1-1111 / 1773 / x-4202 / 1773 x+8558 / 1773 x^{2}\right) \mathrm{H}_{4}+9506 / 3(1-23900 / 42777 / x \\
& \left.-13907 / 14259 x+25964 / 14259 x^{2}\right) \mathrm{H}_{3}-788(1-935 / 1773 / x-4160 / 1773 x
\end{aligned}
$$


$\left.+8558 / 1773 x^{2}\right) \mathrm{H}_{0,0} \zeta_{2}-9506 / 3(1-20684 / 42777 / x-4463 / 6111 x$

$\left.+25964 / 14259 x^{2}\right) \mathrm{H}_{0} \zeta_{2}+3184 / 9\left(1-88 / 199 / x-301 / 398 x+253 / 398 x^{2}\right) \mathrm{H}_{1,-2,0}$

$+959401 / 81\left(1-306237 / 959401 / x-230274 / 959401 x+3294515 / 2878203 x^{2}\right) \mathrm{H}_{0}$

$+146410 / 27\left(1-16284 / 73205 / x-57243 / 73205 x+101818 / 73205 x^{2}\right) \mathrm{H}_{0,0,0}$

$-22004 / 9\left(1-957 / 5501 / x-1450 / 5501 x+9372 / 5501 x^{2}\right) \mathrm{H}_{0} \zeta_{3}$

$-16168 / 9\left(1-341 / 2021 / x+619 / 2021 x+1661 / 2021 x^{2}\right) \mathrm{H}_{-2,2}$

$+23116 / 9\left(1-891 / 5779 / x+998 / 5779 x+4752 / 5779 x^{2}\right) \mathrm{H}_{-2} \zeta_{2}$

$-10360 / 3\left(1-517 / 3885 / x-13 / 2590 x+7337 / 7770 x^{2}\right) \mathrm{H}_{-2,0,0}$

$+1544\left(1-209 / 1737 / x-80 / 579 x+1430 / 1737 x^{2}\right) \mathrm{H}_{-2,-1,0}+63584 / 27(1-201 / 1987 / x$

$\left.-655 / 1987 x+1959 / 1987 x^{2}\right) \mathrm{H}_{-2,0}-15722 / 3(1-31615 / 1910223 / x-477635 / 848988 x$

$\left.+3411235 / 7640892 x^{2}\right)-7568 / 3\left(1+4 / 129 / x+7 / 946 x+89 / 86 x^{2}\right) \mathrm{H}_{-3,0}$

$+1344\left(1+143 / 378 / x+263 / 252 x+253 / 756 x^{2}\right) \mathrm{H}_{-1,-1,2}-3464 / 9(1+198 / 433 / x$

$\left.+2302 / 433 x-2970 / 433 x^{2}\right) \mathrm{H}_{0,0,0,0}-14432 / 9(1+39 / 82 / x+3871 / 3608 x$

$\left.+133 / 328 x^{2}\right) \mathrm{H}_{-1,-1} \zeta_{2}+16532 / 9\left(1+2321 / 4133 / x+4363 / 4133 x+2101 / 4133 x^{2}\right) \mathrm{H}_{-1} \zeta_{3}$

$-2072\left(1+1441 / 2331 / x+788 / 777 x+1408 / 2331 x^{2}\right) \mathrm{H}_{-1,3}+22792 / 9(1+169 / 259 / x$

$\left.+5765 / 5698 x+331 / 518 x^{2}\right) \mathrm{H}_{-1,0} \zeta_{2}-2960 / 3\left(1+121 / 185 / x+x+121 / 185 x^{2}\right) \mathrm{H}_{-1,2,1}$

$-2384 / 3\left(1+99 / 149 / x+x+99 / 149 x^{2}\right) \mathrm{H}_{-1,2,0}+5552 / 3(1+275 / 347 / x+1497 / 1388 x$

$\left.+1001 / 1388 x^{2}\right) \mathrm{H}_{-1,-1,0,0}-302995 / 81(1+244674 / 302995 / x+313876 / 302995 x$

$\left.+19086 / 43285 x^{2}\right) \mathrm{H}_{0,0}-6776 / 3\left(1+72 / 77 / x+157 / 154 x+141 / 154 x^{2}\right) \mathrm{H}_{-1,0,0,0}$

$+4364 / 9\left(1+1045 / 1091 / x-532 / 1091 x-506 / 1091 x^{2}\right) \mathrm{H}_{1} \zeta_{3}-4672 / 9(1+143 / 146 / x$

$\left.+715 / 584 x+451 / 584 x^{2}\right) \mathrm{H}_{-1,-1,-1,0}+5104 / 9(1+30 / 29 / x+368 / 319 x$

$\left.+26 / 29 x^{2}\right) \mathrm{H}_{-1,-2,0}+1848\left(1+7127 / 6237 / x+2074 / 2079 x+7127 / 6237 x^{2}\right) \mathrm{H}_{-1,0,0}$

$+172045 / 81\left(1+243046 / 172045 / x+207847 / 172045 x-26002 / 172045 x^{2}\right) \zeta_{2}$

$+5888 / 9\left(1+3557 / 2208 / x+x+3557 / 2208 x^{2}\right) \mathrm{H}_{-1,2}-172045 / 81(1+70154 / 34409 / x$

$\left.+340219 / 172045 x-26002 / 172045 x^{2}\right) \mathrm{H}_{2}-2096 / 3(1+9677 / 4716 / x+261 / 262 x$

$\left.+9677 / 4716 x^{2}\right) \mathrm{H}_{-1} \zeta_{2}-17752 / 45\left(1+7172 / 2219 / x+6750 / 2219 x-6061 / 4438 x^{2}\right) \zeta_{2}^{2}$

$-800 / 9\left(1+2563 / 300 / x+47 / 50 x+2563 / 300 x^{2}\right) \mathrm{H}_{-1,-1,0}+404 / 9(1+3113 / 101 / x$

$\left.+4613 / 101 x-3927 / 101 x^{2}\right) \mathrm{H}_{2} \zeta_{2}-2752 / 3(1-13 / 43 x) \mathrm{H}_{-3,-1,0}$

$+6464 / 3(1-16 / 101 x) \mathrm{H}_{-3,0,0}+5440 / 3(1-7 / 85 x) \mathrm{H}_{-4,0}-4384 / 3(1-11 / 137 x) \mathrm{H}_{-3} \zeta_{2}$

$+1856(1-3 / 58 x) \zeta_{5}+3008 / 3(1+1 / 47 x) \mathrm{H}_{-3,2}-1760(1+131 / 165 x) \zeta_{2} \zeta_{3}$

$+6656 / 3(1+11 / 13 x) \mathrm{H}_{0,0} \zeta_{3}-1472(1+85 / 69 x) \mathrm{H}_{3,1,0}-1344(1+9 / 7 x) \mathrm{H}_{3,1,1}$

$-4288 / 3(1+87 / 67 x) \mathrm{H}_{3,2}-3616 / 3(1+157 / 113 x) \mathrm{H}_{3,0,0}-5152 / 3(1+35 / 23 x) \mathrm{H}_{4,1}$

$-4384 / 3(1+221 / 137 x) \mathrm{H}_{4,0}+2912 / 3(1+23 / 13 x) \mathrm{H}_{3} \zeta_{2}+1344(1+2 x) \mathrm{H}_{0,0,0} \zeta_{2}$ 
$-1344(1+19 / 9 x) \mathrm{H}_{5}-2336 / 15(1+452 / 73 x) \mathrm{H}_{0} \zeta_{2}^{2}-1920 x \mathrm{H}_{0,0,0,0,0}$

$+p_{\mathrm{gg}}(-x)\left(504 \zeta_{5}+1876 / 3 \zeta_{3}+6464 / 81 \zeta_{2}-1888 / 3 \zeta_{2} \zeta_{3}+5038 / 45 \zeta_{2}^{2}+1376 / 3 \mathrm{H}_{-4,0}\right.$

$-1184 \mathrm{H}_{-3} \zeta_{2}-832 \mathrm{H}_{-3,-1,0}+3872 / 9 \mathrm{H}_{-3,0}+1120 \mathrm{H}_{-3,0,0}+768 \mathrm{H}_{-3,2}-1712 \mathrm{H}_{-2} \zeta_{3}$

$-616 \mathrm{H}_{-2} \zeta_{2}-1984 / 3 \mathrm{H}_{-2,-2,0}+1712 \mathrm{H}_{-2,-1} \zeta_{2}+800 \mathrm{H}_{-2,-1,-1,0}-5104 / 9 \mathrm{H}_{-2,-1,0}$

$-5408 / 3 \mathrm{H}_{-2,-1,0,0}-1312 \mathrm{H}_{-2,-1,2}+536 \mathrm{H}_{-2,0}-1808 \mathrm{H}_{-2,0} \zeta_{2}+704 \mathrm{H}_{-2,0,0}$

$+4144 / 3 \mathrm{H}_{-2,0,0,0}+2992 / 9 \mathrm{H}_{-2,2}+1376 / 3 \mathrm{H}_{-2,2,0}+544 \mathrm{H}_{-2,2,1}+4576 / 3 \mathrm{H}_{-2,3}$

$-7436 / 9 \mathrm{H}_{-1} \zeta_{3}-2144 / 3 \mathrm{H}_{-1} \zeta_{2}+1528 / 15 \mathrm{H}_{-1} \zeta_{2}^{2}-832 \mathrm{H}_{-1,-3,0}+800 \mathrm{H}_{-1,-2,-1,0}$

$+1712 \mathrm{H}_{-1,-2} \zeta_{2}-5192 / 9 \mathrm{H}_{-1,-2,0}-5408 / 3 \mathrm{H}_{-1,-2,0,0}-1312 \mathrm{H}_{-1,-2,2}+6448 / 3 \mathrm{H}_{-1,-1} \zeta_{3}$

$+7568 / 9 \mathrm{H}_{-1,-1} \zeta_{2}+800 \mathrm{H}_{-1,-1,-2,0}-2112 \mathrm{H}_{-1,-1,-1} \zeta_{2}-768 \mathrm{H}_{-1,-1,-1,-1,0}$

$+6688 / 9 \mathrm{H}_{-1,-1,-1,0}+2336 \mathrm{H}_{-1,-1,-1,0,0}+1728 \mathrm{H}_{-1,-1,-1,2}-2144 / 3 \mathrm{H}_{-1,-1,0}$

$+7648 / 3 \mathrm{H}_{-1,-1,0} \zeta_{2}-8624 / 9 \mathrm{H}_{-1,-1,0,0}-2096 \mathrm{H}_{-1,-1,0,0,0}-1408 / 3 \mathrm{H}_{-1,-1,2}$

$-2176 / 3 \mathrm{H}_{-1,-1,2,0}-2432 / 3 \mathrm{H}_{-1,-1,2,1}-6496 / 3 \mathrm{H}_{-1,-1,3}+12928 / 81 \mathrm{H}_{-1,0}$

$-4784 / 3 \mathrm{H}_{-1,0} \zeta_{3}-1936 / 3 \mathrm{H}_{-1,0} \zeta_{2}+2680 / 3 \mathrm{H}_{-1,0,0}-4400 / 3 \mathrm{H}_{-1,0,0} \zeta_{2}$

$+5500 / 9 \mathrm{H}_{-1,0,0,0}+2864 / 3 \mathrm{H}_{-1,0,0,0,0}+1072 / 3 \mathrm{H}_{-1,2}+48 \mathrm{H}_{-1,2} \zeta_{2}+704 / 3 \mathrm{H}_{-1,2,0}$

$+256 \mathrm{H}_{-1,2,0,0}+704 / 3 \mathrm{H}_{-1,2,1}+704 / 3 \mathrm{H}_{-1,2,1,0}+192 \mathrm{H}_{-1,2,1,1}+704 / 3 \mathrm{H}_{-1,2,2}$

$+528 \mathrm{H}_{-1,3}+672 \mathrm{H}_{-1,3,0}+2336 / 3 \mathrm{H}_{-1,3,1}+1328 \mathrm{H}_{-1,4}+120 \mathrm{H}_{0}+5360 / 27 \mathrm{H}_{0} \zeta_{2}$

$+3212 / 9 \mathrm{H}_{0} \zeta_{3}+904 / 15 \mathrm{H}_{0} \zeta_{2}^{2}-6464 / 81 \mathrm{H}_{0,0}+1712 / 3 \mathrm{H}_{0,0} \zeta_{3}+1804 / 9 \mathrm{H}_{0,0} \zeta_{2}-268 \mathrm{H}_{0,0,0}$

$+432 \mathrm{H}_{0,0,0} \zeta_{2}-1364 / 9 \mathrm{H}_{0,0,0,0}-208 \mathrm{H}_{0,0,0,0,0}-496 / 3 \mathrm{H}_{2} \zeta_{3}-836 / 9 \mathrm{H}_{2} \zeta_{2}-112 \mathrm{H}_{2,0} \zeta_{2}$

$-176 / 9 \mathrm{H}_{2,0,0}+64 / 3 \mathrm{H}_{2,0,0,0}-304 / 3 \mathrm{H}_{2,1} \zeta_{2}+88 / 9 \mathrm{H}_{2,1,0}+64 / 3 \mathrm{H}_{2,1,0,0}-88 / 9 \mathrm{H}_{2,2}$

$+64 / 3 \mathrm{H}_{2,3}-536 / 3 \mathrm{H}_{3}-608 / 3 \mathrm{H}_{3} \zeta_{2}-308 / 3 \mathrm{H}_{3,0}-352 / 3 \mathrm{H}_{3,0,0}-352 / 3 \mathrm{H}_{3,1}$

$\left.-352 / 3 \mathrm{H}_{3,1,0}-96 \mathrm{H}_{3,1,1}-352 / 3 \mathrm{H}_{3,2}-1628 / 9 \mathrm{H}_{4}-784 / 3 \mathrm{H}_{4,0}-976 / 3 \mathrm{H}_{4,1}-400 \mathrm{H}_{5}\right)$

$+p_{\mathrm{gg}}(x)\left(-1616486 / 729-480 \zeta_{5}+21994 / 9 \zeta_{3}+170666 / 81 \zeta_{2}-2896 / 3 \zeta_{2} \zeta_{3}\right.$

$-25102 / 45 \zeta_{2}^{2}+1184 / 3 \mathrm{H}_{-4,0}-960 \mathrm{H}_{-3} \zeta_{2}-640 \mathrm{H}_{-3,-1,0}+3520 / 9 \mathrm{H}_{-3,0}+896 \mathrm{H}_{-3,0,0}$

$+640 \mathrm{H}_{-3,2}-2144 / 3 \mathrm{H}_{-2} \zeta_{3}-572 / 3 \mathrm{H}_{-2} \zeta_{2}-544 / 3 \mathrm{H}_{-2,-2,0}+752 \mathrm{H}_{-2,-1} \zeta_{2}$

$+608 / 3 \mathrm{H}_{-2,-1,-1,0}-616 / 3 \mathrm{H}_{-2,-1,0}-2048 / 3 \mathrm{H}_{-2,-1,0,0}-1952 / 3 \mathrm{H}_{-2,-1,2}$

$+13400 / 27 \mathrm{H}_{-2,0}-2320 / 3 \mathrm{H}_{-2,0} \zeta_{2}+2552 / 9 \mathrm{H}_{-2,0,0}+1312 / 3 \mathrm{H}_{-2,0,0,0}+88 \mathrm{H}_{-2,2}$

$+544 / 3 \mathrm{H}_{-2,2,0}+608 / 3 \mathrm{H}_{-2,2,1}+2048 / 3 \mathrm{H}_{-2,3}-191858 / 81 \mathrm{H}_{0}+11792 / 9 \mathrm{H}_{0} \zeta_{3}$

$+6656 / 3 \mathrm{H}_{0} \zeta_{2}-2408 / 5 \mathrm{H}_{0} \zeta_{2}^{2}-130514 / 81 \mathrm{H}_{0,0}+2224 / 3 \mathrm{H}_{0,0} \zeta_{3}+11264 / 9 \mathrm{H}_{0,0} \zeta_{2}$

$-10952 / 9 \mathrm{H}_{0,0,0}+688 \mathrm{H}_{0,0,0} \zeta_{2}-5060 / 9 \mathrm{H}_{0,0,0,0}-272 \mathrm{H}_{0,0,0,0,0}-192268 / 81 \mathrm{H}_{1}$

$+9196 / 9 \mathrm{H}_{1} \zeta_{3}+17236 / 9 \mathrm{H}_{1} \zeta_{2}-296 \mathrm{H}_{1} \zeta_{2}^{2}+1664 / 3 \mathrm{H}_{1,-3,0}-400 \mathrm{H}_{1,-2} \zeta_{2}$

$-1696 / 3 \mathrm{H}_{1,-2,-1,0}+3080 / 9 \mathrm{H}_{1,-2,0}+1792 / 3 \mathrm{H}_{1,-2,0,0}+352 / 3 \mathrm{H}_{1,-2,2}-176374 / 81 \mathrm{H}_{1,0}$

$+2032 / 3 \mathrm{H}_{1,0} \zeta_{3}+12760 / 9 \mathrm{H}_{1,0} \zeta_{2}-47888 / 27 \mathrm{H}_{1,0,0}+688 \mathrm{H}_{1,0,0} \zeta_{2}-9196 / 9 \mathrm{H}_{1,0,0,0}$

$-1456 / 3 \mathrm{H}_{1,0,0,0,0}-19246 / 9 \mathrm{H}_{1,1}+240 \mathrm{H}_{1,1} \zeta_{3}+10736 / 9 \mathrm{H}_{1,1} \zeta_{2}+32 \mathrm{H}_{1,1,-2,0}$ 
$-19484 / 9 \mathrm{H}_{1,1,0}+640 \mathrm{H}_{1,1,0} \zeta_{2}-10384 / 9 \mathrm{H}_{1,1,0,0}-592 \mathrm{H}_{1,1,0,0,0}-2040 \mathrm{H}_{1,1,1}+768 \mathrm{H}_{1,1,1} \zeta_{2}$

$-14696 / 9 \mathrm{H}_{1,1,1,0}-1216 \mathrm{H}_{1,1,1,0,0}-4400 / 3 \mathrm{H}_{1,1,1,1}-1152 \mathrm{H}_{1,1,1,1,0}-960 \mathrm{H}_{1,1,1,1,1}$

$-1152 \mathrm{H}_{1,1,1,2}-14080 / 9 \mathrm{H}_{1,1,2}-1280 \mathrm{H}_{1,1,2,0}-1152 \mathrm{H}_{1,1,2,1}-1024 \mathrm{H}_{1,1,3}-20452 / 9 \mathrm{H}_{1,2}$

$+880 \mathrm{H}_{1,2} \zeta_{2}-14432 / 9 \mathrm{H}_{1,2,0}-3296 / 3 \mathrm{H}_{1,2,0,0}-1584 \mathrm{H}_{1,2,1}-1280 \mathrm{H}_{1,2,1,0}$

$-1152 \mathrm{H}_{1,2,1,1}-1280 \mathrm{H}_{1,2,2}-13816 / 9 \mathrm{H}_{1,3}-3488 / 3 \mathrm{H}_{1,3,0}-1216 \mathrm{H}_{1,3,1}-2480 / 3 \mathrm{H}_{1,4}$

$-177130 / 81 \mathrm{H}_{2}+1568 / 3 \mathrm{H}_{2} \zeta_{3}+3520 / 3 \mathrm{H}_{2} \zeta_{2}+96 \mathrm{H}_{2,-2,0}-16424 / 9 \mathrm{H}_{2,0}$

$+752 \mathrm{H}_{2,0} \zeta_{2}-8360 / 9 \mathrm{H}_{2,0,0}-1616 / 3 \mathrm{H}_{2,0,0,0}-6656 / 3 \mathrm{H}_{2,1}+880 \mathrm{H}_{2,1} \zeta_{2}-14432 / 9 \mathrm{H}_{2,1,0}$

$-3296 / 3 \mathrm{H}_{2,1,0,0}-14344 / 9 \mathrm{H}_{2,1,1}-1280 \mathrm{H}_{2,1,1,0}-1152 \mathrm{H}_{2,1,1,1}-1280 \mathrm{H}_{2,1,2}-13112 / 9 \mathrm{H}_{2,2}$

$-1184 \mathrm{H}_{2,2,0}-1280 \mathrm{H}_{2,2,1}-3104 / 3 \mathrm{H}_{2,3}-60440 / 27 \mathrm{H}_{3}+2432 / 3 \mathrm{H}_{3} \zeta_{2}-3784 / 3 \mathrm{H}_{3,0}$

$-832 \mathrm{H}_{3,0,0}-14344 / 9 \mathrm{H}_{3,1}-3680 / 3 \mathrm{H}_{3,1,0}-1280 \mathrm{H}_{3,1,1}-3680 / 3 \mathrm{H}_{3,2}-11440 / 9 \mathrm{H}_{4}$

$\left.-2704 / 3 \mathrm{H}_{4,0}-3568 / 3 \mathrm{H}_{4,1}-720 \mathrm{H}_{5}\right)+(1-x)\left(-1504 / 3 \mathrm{H}_{-2} \zeta_{3}-1088 / 3 \mathrm{H}_{-2,-2,0}\right.$

$+384 \mathrm{H}_{-2,-1} \zeta_{2}+896 / 3 \mathrm{H}_{-2,-1,-1,0}-2656 / 3 \mathrm{H}_{-2,-1,0,0}-704 / 3 \mathrm{H}_{-2,-1,2}-2560 / 3 \mathrm{H}_{-2,0} \zeta_{2}$

$\left.+3584 / 3 \mathrm{H}_{-2,0,0,0}+704 / 3 \mathrm{H}_{-2,2,0}+256 \mathrm{H}_{-2,2,1}+1952 / 3 \mathrm{H}_{-2,3}\right)+(1+x)\left(896 / 3 \mathrm{H}_{2} \zeta_{3}\right.$

$-128 / 3 \mathrm{H}_{2,-2,0}+512 \mathrm{H}_{2,0} \zeta_{2}-1600 / 3 \mathrm{H}_{2,0,0,0}+2240 / 3 \mathrm{H}_{2,1} \zeta_{2}-3296 / 3 \mathrm{H}_{2,1,0,0}$

$\left.-896 \mathrm{H}_{2,1,1,0}-768 \mathrm{H}_{2,1,1,1}-896 \mathrm{H}_{2,1,2}-992 \mathrm{H}_{2,2,0}-896 \mathrm{H}_{2,2,1}-2144 / 3 \mathrm{H}_{2,3}\right)$

$+\delta(1-x)\left\{14160613 / 2916+4048 / 9 \zeta_{5}-90632 / 27 \zeta_{3}+464 / 3 \zeta_{3}{ }^{2}-196718 / 81 \zeta_{2}\right.$

$\left.\left.+836 \zeta_{2} \zeta_{3}+22501 / 27 \zeta_{2}^{2}-15644 / 105 \zeta_{2}^{3}\right\}\right)$

$+C_{A}^{2} n_{f}\left(-15128 / 27\left(1-4 / 1891 / x^{2}+375 / 1891 / x+1893 / 1891 x+381 / 1891 x^{2}\right) \mathrm{H}_{-1,0}\right.$

$-631 / 27\left(1-264040 / 5679 / x-45427 / 1893 x+132160 / 5679 x^{2}\right) \mathrm{H}_{1}$

$-428 / 9\left(1-848 / 107 / x-481 / 107 x+534 / 107 x^{2}\right) \mathrm{H}_{1,2}-416 / 9(1-102 / 13 / x-215 / 52 x$

$\left.+127 / 26 x^{2}\right) \mathrm{H}_{1,1,1}-92\left(1-2692 / 621 / x-491 / 207 x+1786 / 621 x^{2}\right) \mathrm{H}_{1,1,0}$

$+26362 / 81\left(1-34234 / 13181 / x-62089 / 13181 x+31748 / 13181 x^{2}\right) \zeta_{2}$

$-3284 / 27\left(1-2048 / 821 / x-1513 / 821 x+2594 / 821 x^{2}\right) \mathrm{H}_{2,1}-9536 / 27(1-5465 / 2384 / x$

$\left.-9027 / 4768 x+857 / 596 x^{2}\right) \mathrm{H}_{1,0}-26362 / 81(1-29734 / 13181 / x-39373 / 13181 x$

$\left.+31748 / 13181 x^{2}\right) \mathrm{H}_{2}+1228 / 9\left(1-668 / 307 / x-681 / 307 x+360 / 307 x^{2}\right) \mathrm{H}_{1} \zeta_{2}$

$-460 / 3\left(1-2128 / 1035 / x-31 / 15 x+944 / 345 x^{2}\right) \mathrm{H}_{2,0}-452 / 3(1-656 / 339 / x-571 / 339 x$

$\left.+442 / 339 x^{2}\right) \mathrm{H}_{1,0,0}-10538 / 27\left(1-30380 / 15807 / x-9705 / 5269 x+18482 / 15807 x^{2}\right) \mathrm{H}_{1,1}$

$-13184 / 27\left(1-599 / 412 / x-22057 / 6592 x+3555 / 1648 x^{2}\right) \mathrm{H}_{0,0}+1600 / 9(1-9 / 10 / x+x$

$\left.-87 / 100 x^{2}\right) \mathrm{H}_{-1,-1,0}+64 / 3\left(1-2 / 3 / x-1 / 8 x+37 / 24 x^{2}\right) \mathrm{H}_{1,0,0,0}-248 / 3(1-16 / 31 / x$

$\left.-17 / 31 x+30 / 31 x^{2}\right) \mathrm{H}_{1,0} \zeta_{2}-218 / 45\left(1-48 / 109 / x-1782 / 109 x-804 / 109 x^{2}\right) \zeta_{2}^{2}$

$+3472 / 27\left(1-185 / 434 / x-53 / 7 x+85 / 31 x^{2}\right) \zeta_{3}-1168 / 3(1-226 / 657 / x+218 / 219 x$

$\left.-443 / 1314 x^{2}\right) \mathrm{H}_{-1,0,0}+776 / 9\left(1-28 / 97 / x-109 / 194 x+71 / 97 x^{2}\right) \mathrm{H}_{1,3}$ 
$-1672 / 9(1-3 / 11 / x+206 / 209 x) \mathrm{H}_{0} \zeta_{3}+3968 / 9\left(1-61 / 248 / x+x-119 / 496 x^{2}\right) \mathrm{H}_{-1} \zeta_{2}$ $-2312 / 9\left(1-64 / 289 / x+266 / 289 x-42 / 289 x^{2}\right) \mathrm{H}_{-1,-1,-1,0}-120(1-28 / 135 / x+14 / 15 x$ $\left.-19 / 135 x^{2}\right) \mathrm{H}_{-1,0,0,0}+976 / 3\left(1-12 / 61 / x+225 / 244 x-15 / 122 x^{2}\right) \mathrm{H}_{-1,-1,0,0}$ $+1664 / 9\left(1-5 / 26 / x+191 / 208 x-3 / 26 x^{2}\right) \mathrm{H}_{-1,-2,0}-2524 / 9(1-112 / 631 / x+584 / 631 x$ $\left.-66 / 631 x^{2}\right) \mathrm{H}_{-1,-1} \zeta_{2}+2440 / 9\left(1-43 / 305 / x+569 / 610 x-23 / 305 x^{2}\right) \mathrm{H}_{-1} \zeta_{3}$ $+152\left(1-8 / 57 / x+53 / 57 x-4 / 57 x^{2}\right) \mathrm{H}_{-1,-1,2}-700 / 3(1-2 / 15 / x+761 / 525 x$ $\left.+4 / 35 x^{2}\right) \mathrm{H}_{0,0} \zeta_{2}+904 / 3\left(1-34 / 339 / x+38 / 113 x-20 / 113 x^{2}\right) \mathrm{H}_{-2,-1,0}$ $-776 / 9\left(1-9 / 97 / x+241 / 97 x+71 / 97 x^{2}\right) \mathrm{H}_{2} \zeta_{2}+1268 / 9(1-28 / 317 / x+304 / 317 x$ $\left.-14 / 317 x^{2}\right) \mathrm{H}_{-1,0} \zeta_{2}-352\left(1-8 / 99 / x+x-8 / 99 x^{2}\right) \mathrm{H}_{-1,2}+700 / 3(1-38 / 525 / x$ $\left.+977 / 525 x+4 / 35 x^{2}\right) \mathrm{H}_{4}+1520 / 9(1-6 / 95 / x+40 / 19 x) \mathrm{H}_{0,0,0,0}-968 / 3(1-16 / 363 / x$ $\left.-36 / 121 x-70 / 363 x^{2}\right) \mathrm{H}_{-3,0}-4808 / 27\left(1-26 / 601 / x+740 / 601 x-630 / 601 x^{2}\right) \mathrm{H}_{-2,0}$ $+2056 / 9\left(1-10 / 257 / x+116 / 257 x+32 / 257 x^{2}\right) \mathrm{H}_{2,1,0}+712 / 3(1-3 / 89 / x+677 / 534 x$ $\left.+31 / 267 x^{2}\right) \mathrm{H}_{3,0}+548 / 9\left(1-4 / 137 / x-52 / 137 x+90 / 137 x^{2}\right) \mathrm{H}_{1,1,2}+2368 / 9(1-1 / 37 / x$ $\left.+817 / 592 x+53 / 296 x^{2}\right) \mathrm{H}_{3,1}-1028 / 3\left(1+4 / 257 / x+35 / 257 x-158 / 771 x^{2}\right) \mathrm{H}_{-2,0,0}$ $+740 / 9\left(1+4 / 185 / x-23 / 37 x+64 / 185 x^{2}\right) \mathrm{H}_{1,2,0}+644 / 9(1+4 / 161 / x-76 / 161 x$ $\left.+82 / 161 x^{2}\right) \mathrm{H}_{1,2,1}+2132 / 9\left(1+16 / 533 / x+368 / 533 x+2 / 13 x^{2}\right) \mathrm{H}_{2,2}$ $-412 / 3\left(1+4 / 103 / x+99 / 103 x+8 / 103 x^{2}\right) \mathrm{H}_{-1,3}+3164 / 9(1+6 / 113 / x+4 / 113 x$ $\left.-152 / 791 x^{2}\right) \mathrm{H}_{-2} \zeta_{2}-836 / 9\left(1+12 / 209 / x-271 / 209 x-4 / 11 x^{2}\right) \mathrm{H}_{1,1,0,0}$ $+952 / 9\left(1+2 / 17 / x+571 / 238 x+6 / 17 x^{2}\right) \mathrm{H}_{2,0,0}-1808 / 9(1+19 / 113 / x-43 / 226 x$ $\left.-23 / 113 x^{2}\right) \mathrm{H}_{-2,2}-160 / 3\left(1+1 / 3 / x+x+1 / 3 x^{2}\right) \mathrm{H}_{-1,2,1}-40(1+4 / 9 / x+x$ $\left.+4 / 9 x^{2}\right) \mathrm{H}_{-1,2,0}+608 / 9\left(1+17 / 38 / x-107 / 76 x-33 / 38 x^{2}\right) \mathrm{H}_{1,1} \zeta_{2}$ $+496 / 9\left(1+77 / 62 / x-56 / 31 x-63 / 31 x^{2}\right) \mathrm{H}_{1} \zeta_{3}+208 x \mathrm{H}_{0,0} \zeta_{3}-32 x \mathrm{H}_{0,0,0,0,0}$ $+16306 / 81\left(1+52501 / 8153 / x+78053 / 16306 x-22866 / 8153 x^{2}\right) \mathrm{H}_{0}$ $+544 / 27\left(1+309 / 34 / x+1667 / 34 x-1903 / 68 x^{2}\right) \mathrm{H}_{0,0,0}$ $+28211 / 243\left(1+1582888 / 84633 / x+475721 / 56422 x-1639871 / 169266 x^{2}\right)$ $-88 / 27\left(1+804 / 11 / x+6137 / 22 x-1624 / 11 x^{2}\right) \mathrm{H}_{0} \zeta_{2}+88 / 27(1+830 / 11 / x$ $\left.+4657 / 22 x-1624 / 11 x^{2}\right) \mathrm{H}_{3}-1600 / 9\left(1-23 / 25 x+7 / 100 x^{2}\right) \mathrm{H}_{1,-2,0}$ $+240(1-11 / 15 x) \mathrm{H}_{-3,-1,0}+824 / 9\left(1-62 / 103 x+40 / 103 x^{2}\right) \mathrm{H}_{1,1,1,0}$ $+64\left(1-1 / 2 x+1 / 2 x^{2}\right) \mathrm{H}_{1,1,1,1}-704 / 3(1-17 / 44 x) \mathrm{H}_{-3,0,0}+728 / 3(1-19 / 91 x) \mathrm{H}_{-3} \zeta_{2}$ $-656 / 3(1-1 / 41 x) \mathrm{H}_{-4,0}-368 / 3(1+7 / 23 x) \mathrm{H}_{-3,2}-80(1+31 / 75 x) \mathrm{H}_{0} \zeta_{2}^{2}$ $+224\left(1+4 / 7 x+1 / 7 x^{2}\right) \mathrm{H}_{2,1,1}+376 / 3(1+31 / 47 x) \mathrm{H}_{3} \zeta_{2}-232 / 3(1+21 / 29 x) \mathrm{H}_{3,0,0}$ $-48(1+11 / 9 x) \mathrm{H}_{5}+48(1+4 / 3 x) \mathrm{H}_{0,0,0} \zeta_{2}-232 / 3(1+65 / 29 x) \zeta_{2} \zeta_{3}-56 / 3(1+27 / 7 x) \zeta_{5}$ $+p_{\mathrm{gg}}(-x)\left(-280 / 3 \zeta_{3}-896 / 81 \zeta_{2}-916 / 45 \zeta_{2}^{2}-704 / 9 \mathrm{H}_{-3,0}+112 \mathrm{H}_{-2} \zeta_{2}+928 / 9 \mathrm{H}_{-2,-1,0}\right.$ 
$-80 \mathrm{H}_{-2,0}-128 \mathrm{H}_{-2,0,0}-544 / 9 \mathrm{H}_{-2,2}+1352 / 9 \mathrm{H}_{-1} \zeta_{3}+320 / 3 \mathrm{H}_{-1} \zeta_{2}+944 / 9 \mathrm{H}_{-1,-2,0}$

$-1376 / 9 \mathrm{H}_{-1,-1} \zeta_{2}-1216 / 9 \mathrm{H}_{-1,-1,-1,0}+320 / 3 \mathrm{H}_{-1,-1,0}+1568 / 9 \mathrm{H}_{-1,-1,0,0}$

$+256 / 3 \mathrm{H}_{-1,-1,2}-1792 / 81 \mathrm{H}_{-1,0}+352 / 3 \mathrm{H}_{-1,0} \zeta_{2}-400 / 3 \mathrm{H}_{-1,0,0}-1000 / 9 \mathrm{H}_{-1,0,0,0}$

$-160 / 3 \mathrm{H}_{-1,2}-128 / 3 \mathrm{H}_{-1,2,0}-128 / 3 \mathrm{H}_{-1,2,1}-96 \mathrm{H}_{-1,3}-48 \mathrm{H}_{0}-584 / 9 \mathrm{H}_{0} \zeta_{3}$

$-800 / 27 \mathrm{H}_{0} \zeta_{2}+896 / 81 \mathrm{H}_{0,0}-328 / 9 \mathrm{H}_{0,0} \zeta_{2}+40 \mathrm{H}_{0,0,0}+248 / 9 \mathrm{H}_{0,0,0,0}+152 / 9 \mathrm{H}_{2} \zeta_{2}$

$\left.+32 / 9 \mathrm{H}_{2,0,0}-16 / 9 \mathrm{H}_{2,1,0}+16 / 9 \mathrm{H}_{2,2}+80 / 3 \mathrm{H}_{3}+56 / 3 \mathrm{H}_{3,0}+64 / 3 \mathrm{H}_{3,1}+296 / 9 \mathrm{H}_{4}\right)$

$+p_{\mathrm{gg}}(x)\left(1234307 / 1458-8608 / 27 \zeta_{3}-55712 / 81 \zeta_{2}+4852 / 45 \zeta_{2}^{2}-640 / 9 \mathrm{H}_{-3,0}\right.$

$+104 / 3 \mathrm{H}_{-2} \zeta_{2}+112 / 3 \mathrm{H}_{-2,-1,0}-2000 / 27 \mathrm{H}_{-2,0}-464 / 9 \mathrm{H}_{-2,0,0}-16 \mathrm{H}_{-2,2}$

$+68830 / 81 \mathrm{H}_{0}-992 / 9 \mathrm{H}_{0} \zeta_{3}-4672 / 9 \mathrm{H}_{0} \zeta_{2}+44684 / 81 \mathrm{H}_{0,0}-2048 / 9 \mathrm{H}_{0,0} \zeta_{2}$

$+2776 / 9 \mathrm{H}_{0,0,0}+920 / 9 \mathrm{H}_{0,0,0,0}+67730 / 81 \mathrm{H}_{1}-520 / 9 \mathrm{H}_{1} \zeta_{3}-4544 / 9 \mathrm{H}_{1} \zeta_{2}-560 / 9 \mathrm{H}_{1,-2,0}$

$+56284 / 81 \mathrm{H}_{1,0}-2320 / 9 \mathrm{H}_{1,0} \zeta_{2}+12128 / 27 \mathrm{H}_{1,0,0}+1672 / 9 \mathrm{H}_{1,0,0,0}+6212 / 9 \mathrm{H}_{1,1}$

$-1952 / 9 \mathrm{H}_{1,1} \zeta_{2}+4672 / 9 \mathrm{H}_{1,1,0}+1888 / 9 \mathrm{H}_{1,1,0,0}+512 \mathrm{H}_{1,1,1}+2672 / 9 \mathrm{H}_{1,1,1,0}$

$+800 / 3 \mathrm{H}_{1,1,1,1}+2560 / 9 \mathrm{H}_{1,1,2}+5024 / 9 \mathrm{H}_{1,2}+2624 / 9 \mathrm{H}_{1,2,0}+288 \mathrm{H}_{1,2,1}+2512 / 9 \mathrm{H}_{1,3}$

$+56608 / 81 \mathrm{H}_{2}-640 / 3 \mathrm{H}_{2} \zeta_{2}+1360 / 3 \mathrm{H}_{2,0}+1520 / 9 \mathrm{H}_{2,0,0}+1616 / 3 \mathrm{H}_{2,1}+2624 / 9 \mathrm{H}_{2,1,0}$

$+2608 / 9 \mathrm{H}_{2,1,1}+2384 / 9 \mathrm{H}_{2,2}+14096 / 27 \mathrm{H}_{3}+688 / 3 \mathrm{H}_{3,0}+2608 / 9 \mathrm{H}_{3,1}+2080 / 9 \mathrm{H}_{4}$ )

$+(1-x)\left(488 / 3 \mathrm{H}_{-2} \zeta_{3}+368 / 3 \mathrm{H}_{-2,-2,0}-544 / 3 \mathrm{H}_{-2,-1} \zeta_{2}-544 / 3 \mathrm{H}_{-2,-1,-1,0}\right.$

$+216 \mathrm{H}_{-2,-1,0,0}+272 / 3 \mathrm{H}_{-2,-1,2}+224 / 3 \mathrm{H}_{-2,0} \zeta_{2}-80 \mathrm{H}_{-2,0,0,0}+16 / 3 \mathrm{H}_{-2,2,0}$

$\left.-152 / 3 \mathrm{H}_{-2,3}+16 / 3 \mathrm{H}_{3,1,0}-16 / 3 \mathrm{H}_{3,2}\right)+(1+x)\left(464 / 3 \mathrm{H}_{2} \zeta_{3}-224 / 3 \mathrm{H}_{2,-2,0}\right.$

$+176 / 3 \mathrm{H}_{2,0} \zeta_{2}-32 \mathrm{H}_{2,0,0,0}+304 / 3 \mathrm{H}_{2,1} \zeta_{2}-232 / 3 \mathrm{H}_{2,1,0,0}+32 / 3 \mathrm{H}_{2,1,1,0}-32 / 3 \mathrm{H}_{2,1,2}$

$\left.+40 / 3 \mathrm{H}_{2,2,0}-104 / 3 \mathrm{H}_{2,3}-24 \mathrm{H}_{4,0}-40 \mathrm{H}_{4,1}\right)+\delta(1-x)\{-1988899 / 972$

$\left.\left.+1064 / 9 \zeta_{5}+6794 / 27 \zeta_{3}+70774 / 81 \zeta_{2}-24 \zeta_{2} \zeta_{3}-28526 / 135 \zeta_{2}^{2}\right\}\right)$

$+C_{F} C_{A} n_{f}\left(11528 / 9\left(1-4 / 1441 / x^{2}+122 / 1441 / x+1384 / 1441 x+69 / 1441 x^{2}\right) \mathrm{H}_{-1,0}\right.$

$-56 / 9\left(1-309 / 7 / x-78 / 7 x+50 x^{2}\right) \mathrm{H}_{1,1,0}-340 / 27(1-1338 / 85 / x-643 / 17 x$

$\left.+3304 / 85 x^{2}\right) \mathrm{H}_{2,0}-68 / 3\left(1-1450 / 153 / x-41 / 17 x+98 / 9 x^{2}\right) \mathrm{H}_{1,1,1}$

$+2362 / 27\left(1-2384 / 1181 / x+3209 / 1181 x+7616 / 1181 x^{2}\right) \mathrm{H}_{0} \zeta_{2}$

$-2362 / 27\left(1-2256 / 1181 / x-1843 / 1181 x+7616 / 1181 x^{2}\right) \mathrm{H}_{3}+800 / 3(1-67 / 75 / x+x$

$\left.-67 / 75 x^{2}\right) \mathrm{H}_{-1,-1} \zeta_{2}-169601 / 243(1-121601 / 169601 / x-89143 / 169601 x$

$\left.+276912 / 169601 x^{2}\right) \mathrm{H}_{0}-3952 / 9\left(1-141 / 247 / x+265 / 247 x-123 / 247 x^{2}\right) \mathrm{H}_{-1,-1,0}$

$-904 / 3\left(1-58 / 113 / x+x-58 / 113 x^{2}\right) \mathrm{H}_{-1} \zeta_{3}+6128 / 9(1-466 / 1149 / x+847 / 766 x$

$\left.-689 / 2298 x^{2}\right) \mathrm{H}_{-1,0,0}-1400 / 3\left(1-212 / 525 / x+x-212 / 525 x^{2}\right) \mathrm{H}_{-1,-1,0,0}$

$-6712 / 9\left(1-259 / 839 / x+911 / 839 x-187 / 839 x^{2}\right) \mathrm{H}_{-1} \zeta_{2}+544(1-46 / 153 / x+x$

$\left.-46 / 153 x^{2}\right) \mathrm{H}_{-1,-1,-1,0}-1136 / 3\left(1-20 / 71 / x+x-20 / 71 x^{2}\right) \mathrm{H}_{-1,-2,0}$ 
$-23150 / 27\left(1-2664 / 11575 / x-5048 / 11575 x+9784 / 11575 x^{2}\right) \mathrm{H}_{0,0,0}$ $+4736 / 9\left(1-59 / 296 / x+323 / 296 x-4 / 37 x^{2}\right) \mathrm{H}_{-1,2}-1712 / 3(1-20 / 107 / x+66 / 107 x$ $\left.-68 / 321 x^{2}\right) \mathrm{H}_{-2,-1,0}-664\left(1-44 / 249 / x-20 / 83 x-100 / 747 x^{2}\right) \mathrm{H}_{-2} \zeta_{2}$ $+1136 / 3\left(1-12 / 71 / x-63 / 71 x-16 / 213 x^{2}\right) \mathrm{H}_{-2,2}+656(1-44 / 369 / x+1 / 246 x$ $\left.-26 / 369 x^{2}\right) \mathrm{H}_{-2,0,0}+1904 / 3\left(1-8 / 119 / x-39 / 119 x-12 / 119 x^{2}\right) \mathrm{H}_{-3,0}$ $+4384 / 9\left(1-8 / 411 / x+421 / 548 x-475 / 822 x^{2}\right) \mathrm{H}_{-2,0}+1184 / 3(1+4 / 111 / x-x$ $\left.-4 / 111 x^{2}\right) \mathrm{H}_{1,-2,0}+13612 / 45\left(1+352 / 3403 / x-2282 / 3403 x-776 / 3403 x^{2}\right) \zeta_{2}^{2}$ $+22804 / 27\left(1+4697 / 34206 / x-9101 / 11402 x-4828 / 17103 x^{2}\right) \mathrm{H}_{1,1}$ $+20020 / 27\left(1+159 / 910 / x-7283 / 10010 x-156 / 385 x^{2}\right) \mathrm{H}_{1,0}$ $+384329 / 243\left(1+455021 / 2305974 / x-19253 / 69878 x-486427 / 1152987 x^{2}\right)$ $+3140 / 9(1+192 / 785 / x+1919 / 785 x) \mathrm{H}_{0,0,0,0}-78058 / 81(1+10992 / 39029 / x$ $\left.-68023 / 39029 x-9282 / 39029 x^{2}\right) \zeta_{2}+2960 / 9\left(1+12 / 37 / x+104 / 185 x-54 / 185 x^{2}\right) \mathrm{H}_{3,1}$ $-4496 / 9\left(1+92 / 281 / x-83 / 562 x-76 / 281 x^{2}\right) \mathrm{H}_{2} \zeta_{2}+3976 / 9(1+172 / 497 / x$ $\left.-13 / 497 x-152 / 497 x^{2}\right) \mathrm{H}_{2,0,0}+2752 / 9\left(1+15 / 43 / x+197 / 344 x-12 / 43 x^{2}\right) \mathrm{H}_{3,0}$ $+3100 / 9\left(1+288 / 775 / x+1339 / 775 x-168 / 775 x^{2}\right) \mathrm{H}_{4}+78058 / 81(1+15384 / 39029 / x$ $\left.-18199 / 39029 x-9282 / 39029 x^{2}\right) \mathrm{H}_{2}-1112 / 3\left(1+68 / 139 / x-x-68 / 139 x^{2}\right) \mathrm{H}_{1,1} \zeta_{2}$ $-3100 / 9\left(1+384 / 775 / x+1807 / 775 x-168 / 775 x^{2}\right) \mathrm{H}_{0,0} \zeta_{2}+1928 / 9(1+124 / 241 / x$ $\left.+115 / 241 x-84 / 241 x^{2}\right) \mathrm{H}_{2,2}+344 / 3\left(1+24 / 43 / x+x+24 / 43 x^{2}\right) \mathrm{H}_{-1,0,0,0}$ $+2024 / 9\left(1+148 / 253 / x+97 / 253 x-100 / 253 x^{2}\right) \mathrm{H}_{2,1,0}+664 / 3(1+148 / 249 / x-x$ $\left.-148 / 249 x^{2}\right) \mathrm{H}_{1,3}+1648 / 9\left(1+64 / 103 / x+34 / 103 x-40 / 103 x^{2}\right) \mathrm{H}_{2,1,1}$ $+280\left(1+40 / 63 / x-x-40 / 63 x^{2}\right) \mathrm{H}_{1,1,0,0}-640 / 3\left(1+5 / 6 / x-x-5 / 6 x^{2}\right) \mathrm{H}_{1,0} \zeta_{2}$ $-760 / 3\left(1+50 / 57 / x-x-50 / 57 x^{2}\right) \mathrm{H}_{1} \zeta_{3}+152 / 3\left(1+52 / 57 / x+x+52 / 57 x^{2}\right) \mathrm{H}_{-1,3}$ $+4468 / 27\left(1+1062 / 1117 / x+2947 / 1117 x-2918 / 1117 x^{2}\right) \mathrm{H}_{2,1}+296 / 3(1+112 / 111 / x$ $\left.-x-112 / 111 x^{2}\right) \mathrm{H}_{1,1,2}-2888 / 9\left(1+1114 / 1083 / x-306 / 361 x-1369 / 1083 x^{2}\right) \mathrm{H}_{1} \zeta_{2}$ $+224 / 3\left(1+22 / 21 / x+x+22 / 21 x^{2}\right) \mathrm{H}_{-1,2,1}+31550 / 81(1+16644 / 15775 / x$ $\left.-9451 / 3155 x-2784 / 15775 x^{2}\right) \mathrm{H}_{0,0}+136\left(1+200 / 153 / x-x-200 / 153 x^{2}\right) \mathrm{H}_{1,0,0,0}$ $+8\left(1+4 / 3 / x-x-4 / 3 x^{2}\right)\left(22 / 3 \mathrm{H}_{1,1,1,1}+7 \mathrm{H}_{1,2,1}\right)+160 / 3\left(1+4 / 3 / x+x+4 / 3 x^{2}\right) \mathrm{H}_{-1,2,0}$ $-164\left(1+592 / 369 / x+455 / 123 x-64 / 123 x^{2}\right) \mathrm{H}_{0} \zeta_{3}+1792 / 9(1+11 / 6 / x$ $\left.-143 / 224 x-1475 / 672 x^{2}\right) \mathrm{H}_{1,0,0}+152 / 3\left(1+112 / 57 / x-x-112 / 57 x^{2}\right) \mathrm{H}_{1,1,1,0}$ $+48\left(1+2 / x-x-2 x^{2}\right) \mathrm{H}_{1,2,0}+304 / 3\left(1+691 / 342 / x-41 / 114 x-500 / 171 x^{2}\right) \mathrm{H}_{1,2}$ $-4262 / 27\left(1+6900 / 2131 / x-16385 / 2131 x-1952 / 2131 x^{2}\right) \zeta_{3}$ $+15112 / 81\left(1+33767 / 7556 / x-4061 / 7556 x-29945 / 7556 x^{2}\right) \mathrm{H}_{1}+16 / 3(1+88 / 3 / x$ $\left.+x+88 / 3 x^{2}\right) \mathrm{H}_{-1,-1,2}-256 / 3(1-155 / 16 x) \mathrm{H}_{5}+256 / 3(1-149 / 16 x) \mathrm{H}_{0,0,0} \zeta_{2}$ 
$-480(1-47 / 30 x) \mathrm{H}_{0,0,0,0,0}-544(1-14 / 17 x) \mathrm{H}_{-3,-1,0}+304(1-9 / 19 x) \mathrm{H}_{-3,0,0}$ $-400(1-8 / 25 x) \mathrm{H}_{-3} \zeta_{2}+1024 / 3(1+3 / 32 x) \mathrm{H}_{-4,0}-448(1+31 / 168 x) \zeta_{5}$

$+128(1+3 / 4 x) \mathrm{H}_{-3,2}+560 / 3(1+437 / 350 x) \mathrm{H}_{0} \zeta_{2}^{2}-1280 / 3(1+107 / 80 x) \mathrm{H}_{3} \zeta_{2}$ $+384(1+25 / 18 x) \mathrm{H}_{3,0,0}+1232 / 3(1+138 / 77 x) \zeta_{2} \zeta_{3}+560 / 3(1+71 / 35 x) \mathrm{H}_{3,1,0}$ $+464 / 3(1+65 / 29 x) \mathrm{H}_{3,2}+416 / 3(1+31 / 13 x) \mathrm{H}_{3,1,1}-496 / 3(1+163 / 31 x) \mathrm{H}_{0,0} \zeta_{3}$ $+112(1+39 / 7 x) \mathrm{H}_{4,1}+208 / 3(1+109 / 13 x) \mathrm{H}_{4,0}+p_{\mathrm{gg}}(-x)\left(-224 / 5 \zeta_{2}^{2}-128 / 3 \mathrm{H}_{-3,0}\right.$ $\left.-24 \mathrm{H}_{0}-128 / 3 \mathrm{H}_{0} \zeta_{3}-64 / 3 \mathrm{H}_{0,0} \zeta_{2}+64 / 3 \mathrm{H}_{0,0,0,0}\right)+p_{\mathrm{gg}}(x)\left(7810 / 27-2152 / 9 \zeta_{3}\right.$ $-100 / 3 \zeta_{2}+32 / 15 \zeta_{2}^{2}-256 / 3 \mathrm{H}_{-2} \zeta_{2}+128 / 3 \mathrm{H}_{-2,0,0}+256 / 3 \mathrm{H}_{-2,2}+2020 / 9 \mathrm{H}_{0}$ $-64 \mathrm{H}_{0} \zeta_{3}-176 / 9 \mathrm{H}_{0} \zeta_{2}+24 \mathrm{H}_{0,0}+64 \mathrm{H}_{0,0} \zeta_{2}-64 / 3 \mathrm{H}_{0,0,0,0}+598 / 3 \mathrm{H}_{1}-128 \mathrm{H}_{1} \zeta_{3}$ $\left.+32 \mathrm{H}_{1,0}+32 \mathrm{H}_{1,1}+100 / 3 \mathrm{H}_{2}-176 / 9 \mathrm{H}_{2,0}+176 / 9 \mathrm{H}_{3}-128 / 3 \mathrm{H}_{4}\right)$

$+(1-x)\left(-1120 / 3 \mathrm{H}_{-2} \zeta_{3}-992 / 3 \mathrm{H}_{-2,-2,0}+1408 / 3 \mathrm{H}_{-2,-1} \zeta_{2}+1472 / 3 \mathrm{H}_{-2,-1,-1,0}\right.$ $-496 \mathrm{H}_{-2,-1,0,0}-224 \mathrm{H}_{-2,-1,2}-128 / 3 \mathrm{H}_{-2,0} \zeta_{2}-16 / 3 \mathrm{H}_{-2,0,0,0}-64 \mathrm{H}_{-2,2,0}$ $\left.-64 \mathrm{H}_{-2,2,1}-16 \mathrm{H}_{-2,3}\right)+(1+x)\left(-128 / 3 \mathrm{H}_{-1,0} \zeta_{2}-416 \mathrm{H}_{2} \zeta_{3}+640 / 3 \mathrm{H}_{2,-2,0}\right.$ $-992 / 3 \mathrm{H}_{2,0} \zeta_{2}+880 / 3 \mathrm{H}_{2,0,0,0}-1232 / 3 \mathrm{H}_{2,1} \zeta_{2}+1072 / 3 \mathrm{H}_{2,1,0,0}+400 / 3 \mathrm{H}_{2,1,1,0}$ $\left.+352 / 3 \mathrm{H}_{2,1,1,1}+496 / 3 \mathrm{H}_{2,1,2}+128 \mathrm{H}_{2,2,0}+112 \mathrm{H}_{2,2,1}+272 \mathrm{H}_{2,3}\right)$ $\left.+\delta(1-x)\left\{-411137 / 324+200 \zeta_{5}+6664 / 9 \zeta_{3}+2128 / 9 \zeta_{2}-128 \zeta_{2} \zeta_{3}+176 / 45 \zeta_{2}^{2}\right\}\right)$ $+C_{F}^{2} n_{f}\left(-12016 / 9\left(1-2 / 2253 / x^{2}+5 / 1502 / x+4505 / 4506 x+3 / 751 x^{2}\right) \mathrm{H}_{-1,0}\right.$ $-16 / 3\left(1-13 / x-5 x+17 x^{2}\right) \mathrm{H}_{1,0,0}+44 / 3\left(1-32 / 11 / x+70 / 11 x-128 / 33 x^{2}\right) \mathrm{H}_{0,0,0,0}$ $-256 / 3\left(1-19 / 12 / x+x-19 / 12 x^{2}\right) \mathrm{H}_{-1,0,0,0}+592 / 3(1-172 / 111 / x+x$ $\left.-172 / 111 x^{2}\right) \mathrm{H}_{-1,-1,0,0}-296 / 3\left(1-56 / 37 / x-94 / 37 x-112 / 111 x^{2}\right) \mathrm{H}_{0} \zeta_{3}$ $+452 / 3\left(1-280 / 339 / x-137 / 113 x+352 / 339 x^{2}\right) \mathrm{H}_{1} \zeta_{2}+704 / 3(1-20 / 33 / x+x$ $\left.-20 / 33 x^{2}\right) \mathrm{H}_{-1,-2,0}-320\left(1-8 / 15 / x+x-8 / 15 x^{2}\right) \mathrm{H}_{-1,-1,-1,0}-192(1-4 / 9 / x-13 / 6 x$ $\left.-2 / 3 x^{2}\right) \mathrm{H}_{-2,2}+352\left(1-4 / 11 / x-10 / 11 x-20 / 33 x^{2}\right) \mathrm{H}_{-2} \zeta_{2}-592 / 3(1-12 / 37 / x$ $\left.+19 / 74 x-14 / 37 x^{2}\right) \mathrm{H}_{0,0} \zeta_{2}+320\left(1-4 / 15 / x+3 / 5 x-8 / 15 x^{2}\right) \mathrm{H}_{-2,-1,0}$ $+1424 / 3\left(1-70 / 267 / x+79 / 89 x-100 / 267 x^{2}\right) \mathrm{H}_{-1,-1,0}-1216 / 3(1-4 / 19 / x-21 / 76 x$ $\left.-37 / 57 x^{2}\right) \mathrm{H}_{-2,0,0}+712\left(1-122 / 801 / x+257 / 267 x-152 / 801 x^{2}\right) \mathrm{H}_{-1} \zeta_{2}$ $+592 / 3\left(1-4 / 37 / x+51 / 74 x-14 / 37 x^{2}\right) \mathrm{H}_{4}-1424 / 3\left(1-26 / 267 / x+x-26 / 267 x^{2}\right) \mathrm{H}_{-1,2}$ $-1216 / 3\left(1-2 / 19 / x-4 / 19 x-26 / 57 x^{2}\right) \mathrm{H}_{-3,0}-9104 / 9(1-48 / 569 / x+554 / 569 x$ $\left.-63 / 569 x^{2}\right) \mathrm{H}_{-1,0,0}-136\left(1-4 / 51 / x-x+4 / 51 x^{2}\right) \mathrm{H}_{1,3}+2141 / 9(1+264 / 2141 / x$ $\left.+42253 / 6423 x-9776 / 6423 x^{2}\right) \mathrm{H}_{0,0}+680 / 3\left(1+16 / 85 / x+77 / 85 x-28 / 85 x^{2}\right) \mathrm{H}_{3,1}$ $+48631 / 162\left(1+9513 / 48631 / x-1270 / 48631 x-58494 / 48631 x^{2}\right)+200(1+16 / 75 / x$ $\left.+24 / 25 x-8 / 25 x^{2}\right) \mathrm{H}_{3,0}+7442 / 27\left(1+836 / 3721 / x+773 / 7442 x-18254 / 11163 x^{2}\right) \mathrm{H}_{0}$ 
$+136\left(1+4 / 17 / x-x-4 / 17 x^{2}\right) \mathrm{H}_{1,1} \zeta_{2}+488 / 3\left(1+16 / 61 / x+49 / 61 x-52 / 183 x^{2}\right) \mathrm{H}_{2,1,0}$ $+200\left(1+4 / 15 / x+58 / 75 x-56 / 225 x^{2}\right) \mathrm{H}_{2,1,1}+216\left(1+8 / 27 / x+7 / 9 x-20 / 81 x^{2}\right) \mathrm{H}_{2,2}$ $-56\left(1+8 / 21 / x+33 / 7 x+4 / 7 x^{2}\right) \mathrm{H}_{2} \zeta_{2}+376 / 3\left(1+68 / 141 / x-x-68 / 141 x^{2}\right) \mathrm{H}_{1,0} \zeta_{2}$ $+64 / 3\left(1+1 / 2 / x+39 / 8 x-1 / 2 x^{2}\right) \mathrm{H}_{2,0,0}+7141 / 27(1+11065 / 21423 / x+1340 / 7141 x$ $\left.-36508 / 21423 x^{2}\right) \mathrm{H}_{1}+220\left(1+284 / 495 / x+17 / 33 x-472 / 495 x^{2}\right) \mathrm{H}_{2,0}$ $+78\left(1+272 / 351 / x+911 / 117 x-1312 / 351 x^{2}\right) \mathrm{H}_{3}-78(1+272 / 351 / x+445 / 39 x$ $\left.-1312 / 351 x^{2}\right) \mathrm{H}_{0} \zeta_{2}+416 / 3\left(1+71 / 78 / x+29 / 52 x-22 / 13 x^{2}\right) \mathrm{H}_{2,1}+152 / 3(1+52 / 57 / x$ $\left.-x-52 / 57 x^{2}\right) \mathrm{H}_{1,1,1,0}-112\left(1+20 / 21 / x+x+20 / 21 x^{2}\right)\left(2 \mathrm{H}_{-1,-1,2}-\mathrm{H}_{-1,3}\right)$ $+64\left(1+1 / x-x-x^{2}\right) \mathrm{H}_{1,2,0}+856 / 9\left(1+374 / 321 / x+1379 / 428 x-1321 / 321 x^{2}\right) \mathrm{H}_{2}$ $-856 / 9\left(1+389 / 321 / x+22157 / 1284 x-1321 / 321 x^{2}\right) \zeta_{2}+224 / 3(1+10 / 7 x) \zeta_{5}$ $+8\left(1+4 / 3 / x-x-4 / 3 x^{2}\right)\left(\mathrm{H}_{1,1,0,0}+14 / 3 \mathrm{H}_{1,1,1,1}+7 \mathrm{H}_{1,2,1}\right)-368 / 3(1+100 / 69 / x+x$ $\left.+100 / 69 x^{2}\right) \mathrm{H}_{-1,0} \zeta_{2}+820 / 9\left(1+316 / 205 / x-49 / 205 x-472 / 205 x^{2}\right) \mathrm{H}_{1,1,0}-576 \mathrm{H}_{-4,0}$ $-128 / 3\left(1+11 / 6 / x-x-11 / 6 x^{2}\right) \mathrm{H}_{1,0,0,0}+812 / 9(1+384 / 203 / x-59 / 203 x$ $\left.-528 / 203 x^{2}\right) \mathrm{H}_{1,1,1}+260 / 3\left(1+28 / 13 / x-21 / 65 x-184 / 65 x^{2}\right) \mathrm{H}_{1,2}+24(1+20 / 9 / x$ $\left.-x-20 / 9 x^{2}\right) \mathrm{H}_{1,1,2}+686 / 9\left(1+3208 / 1029 / x+241 / 343 x-4960 / 1029 x^{2}\right) \mathrm{H}_{1,0}$ $+64\left(1+14 / 3 / x+x+14 / 3 x^{2}\right) \mathrm{H}_{-1,-1} \zeta_{2}+188 / 15\left(1+808 / 141 / x+577 / 47 x+44 / 47 x^{2}\right) \zeta_{2}^{2}$ $+40 / 3\left(1+28 / 3 / x-x-28 / 3 x^{2}\right) \mathrm{H}_{1} \zeta_{3}+50 / 9\left(1+88 / 5 / x-4609 / 25 x+1144 / 25 x^{2}\right) \zeta_{3}$ $+70 / 3\left(1+424 / 45 / x+667 / 105 x-5284 / 315 x^{2}\right) \mathrm{H}_{1,1}-8\left(1+92 / 3 / x+x+92 / 3 x^{2}\right) \mathrm{H}_{-1} \zeta_{3}$ $+32 / 3(1-11 x) \zeta_{2} \zeta_{3}-304 / 3(1-14 / 19 x) \mathrm{H}_{0} \zeta_{2}^{2}+1600 / 3(1-16 / 25 x) \mathrm{H}_{-3,-1,0}$ $-608(1-13 / 57 x) \mathrm{H}_{-3,0,0}+1568 / 3(1-10 / 49 x) \mathrm{H}_{-3} \zeta_{2}-712 / 3(1-15 / 89 x) \mathrm{H}_{0,0} \zeta_{3}$ $+320 / 3(1+1 / 10 x) \mathrm{H}_{3} \zeta_{2}-160 / 3(1+1 / 5 x) \mathrm{H}_{3,0,0}-256(1+1 / 4 x) \mathrm{H}_{-3,2}$ $-7120 / 9\left(1+159 / 445 x-20 / 89 x^{2}\right) \mathrm{H}_{-2,0}+946 / 9\left(1+4570 / 473 x-912 / 473 x^{2}\right) \mathrm{H}_{0,0,0}$ $+p_{\mathrm{gg}}(-x)\left(224 / 5 \zeta_{2}^{2}+128 / 3 \mathrm{H}_{-3,0}+128 / 3 \mathrm{H}_{0} \zeta_{3}+64 / 3 \mathrm{H}_{0,0} \zeta_{2}-64 / 3 \mathrm{H}_{0,0,0,0}\right)$ $+p_{\mathrm{gg}}(x)\left(-1-128 / 15 \zeta_{2}^{2}+256 / 3 \mathrm{H}_{-2} \zeta_{2}-128 / 3 \mathrm{H}_{-2,0,0}-256 / 3 \mathrm{H}_{-2,2}-64 \mathrm{H}_{0,0} \zeta_{2}\right.$ $\left.-64 \mathrm{H}_{0} \zeta_{3}+64 / 3 \mathrm{H}_{0,0,0,0}+128 / 3 \mathrm{H}_{4}\right)+(1-x)\left(336 \mathrm{H}_{-2} \zeta_{3}+896 / 3 \mathrm{H}_{-2,-2,0}-384 \mathrm{H}_{-2,-1} \zeta_{2}\right.$ $-384 \mathrm{H}_{-2,-1,-1,0}+1504 / 3 \mathrm{H}_{-2,-1,0,0}+192 \mathrm{H}_{-2,-1,2}+544 / 3 \mathrm{H}_{-2,0} \zeta_{2}-640 / 3 \mathrm{H}_{-2,0,0,0}$ $\left.-96 \mathrm{H}_{-2,3}-256 \mathrm{H}_{1,-2,0}\right)+(1+x)\left(-616 / 3 \mathrm{H}_{0,0,0} \zeta_{2}+120 \mathrm{H}_{0,0,0,0,0}+560 / 3 \mathrm{H}_{2} \zeta_{3}+616 / 3 \mathrm{H}_{5}\right.$ $-128 \mathrm{H}_{2,-2,0}+144 \mathrm{H}_{2,0} \zeta_{2}-128 \mathrm{H}_{2,0,0,0}+368 / 3 \mathrm{H}_{2,1} \zeta_{2}-16 / 3 \mathrm{H}_{2,1,0,0}+80 \mathrm{H}_{2,1,1,0}$ $+224 / 3 \mathrm{H}_{2,1,1,1}+208 / 3 \mathrm{H}_{2,1,2}+320 / 3 \mathrm{H}_{2,2,0}+112 \mathrm{H}_{2,2,1}-176 / 3 \mathrm{H}_{2,3}+128 \mathrm{H}_{3,1,0}$ $\left.\left.+448 / 3 \mathrm{H}_{3,1,1}+160 \mathrm{H}_{3,2}+176 \mathrm{H}_{4,0}+640 / 3 \mathrm{H}_{4,1}\right)+\delta(1-x)\left\{751 / 9-400 \zeta_{5}+740 / 3 \zeta_{3}\right\}\right)$ $+C_{A} n_{f}^{2}\left(4498 / 81\left(1+1128 / 2249 / x+131 / 2249 x+524 / 2249 x^{2}\right)\left(\zeta_{2}-\mathrm{H}_{2}\right)\right.$

$-1286 / 27\left(1+1024 / 1929 / x-99 / 643 x+524 / 1929 x^{2}\right) \mathrm{H}_{1,1}-1264 / 27(1+269 / 474 / x$ 


$$
\begin{aligned}
& \left.-47 / 316 x+59 / 237 x^{2}\right) \mathrm{H}_{1,0}-3524 / 81\left(1+642 / 881 / x+428 / 881 x+56 / 881 x^{2}\right) \mathrm{H}_{0,0} \\
& -217 / 3\left(1+18169 / 17577 / x+104 / 837 x-262 / 17577 x^{2}\right) \mathrm{H}_{1}-2170 / 27(1+1208 / 1085 / x \\
& \left.+214 / 651 x+1 / 9765 x^{2}\right) \mathrm{H}_{0}-1112 / 27\left(1-68 / 139 x+69 / 139 x^{2}\right) \zeta_{3} \\
& -22751 / 243\left(1+34312 / 22751 / x+17705 / 45502 x-10817 / 45502 x^{2}\right) \\
& +16 / 3\left(1-1 / 2 x+1 / 2 x^{2}\right)\left(16 / 3 \mathrm{H}_{1} \zeta_{2}-10 / 3 \mathrm{H}_{1,0,0}-4 \mathrm{H}_{1,1,0}-14 / 3 \mathrm{H}_{1,1,1}-16 / 3 \mathrm{H}_{1,2}\right) \\
& -704 / 27\left(1-19 / 44 x+21 / 44 x^{2}\right) \mathrm{H}_{2,1}-544 / 27\left(1-11 / 34 x+15 / 34 x^{2}\right) \mathrm{H}_{2,0} \\
& +568 / 27\left(1-19 / 71 x+30 / 71 x^{2}\right)\left(\mathrm{H}_{0} \zeta_{2}-\mathrm{H}_{3}\right)+p_{\mathrm{gg}}(x)\left(-138493 / 1458-296 / 27 \zeta_{3}\right. \\
& +584 / 9 \zeta_{2}-6740 / 81 \mathrm{H}_{0}+256 / 9 \mathrm{H}_{0} \zeta_{2}-56 \mathrm{H}_{0,0}-176 / 9 \mathrm{H}_{0,0,0}-6652 / 81 \mathrm{H}_{1}+304 / 9 \mathrm{H}_{1} \zeta_{2} \\
& -584 / 9 \mathrm{H}_{1,0}-256 / 9 \mathrm{H}_{1,0,0}-584 / 9 \mathrm{H}_{1,1}-272 / 9 \mathrm{H}_{1,1,0}-32 \mathrm{H}_{1,1,1}-304 / 9 \mathrm{H}_{1,2} \\
& \left.-584 / 9 \mathrm{H}_{2}-256 / 9 \mathrm{H}_{2,0}-32 \mathrm{H}_{2,1}-256 / 9 \mathrm{H}_{3}\right)-200 / 27 \mathrm{H}_{0,0,0}(1+x) \\
& \left.+\delta(1-x)\left\{174769 / 972+404 / 9 \zeta_{3}-6740 / 81 \zeta_{2}+608 / 45 \zeta_{2}^{2}\right\}\right) \\
& +C_{F} n_{f}^{2}\left(-184 / 45(1+x) \zeta_{2}^{2}+2684 / 27\left(1+96 / 671 / x+515 / 671 x-16 / 671 x^{2}\right)\left(\mathrm{H}_{0} \zeta_{2}-\mathrm{H}_{3}\right)\right. \\
& +3092 / 27\left(1+136 / 773 / x+71 / 773 x+244 / 773 x^{2}\right) \zeta_{3}-3508 / 27(1+168 / 877 / x \\
& \left.+826 / 877 x-56 / 877 x^{2}\right) \mathrm{H}_{0,0,0}-1406 / 3(1+38524 / 170829 / x-3974 / 6327 x \\
& \left.-53617 / 170829 x^{2}\right)-1240 / 27\left(1+36 / 155 / x+134 / 155 x-16 / 155 x^{2}\right) \mathrm{H}_{2,1} \\
& -68290 / 243\left(1+1941 / 6829 / x+4931 / 34145 x-11746 / 34145 x^{2}\right) \mathrm{H}_{0} \\
& -14240 / 81\left(1+57 / 178 / x+1177 / 1780 x-211 / 890 x^{2}\right) \mathrm{H}_{0,0}-1016 / 27(1+48 / 127 / x \\
& \left.+166 / 127 x-32 / 127 x^{2}\right) \mathrm{H}_{2,0}+56 / 9\left(1+8 / 21 / x-9 / 7 x-20 / 21 x^{2}\right)\left(\mathrm{H}_{1} \zeta_{2}-\mathrm{H}_{1,2}\right) \\
& +8020 / 81\left(1+948 / 2005 / x+230 / 401 x-160 / 401 x^{2}\right)\left(\zeta_{2}-\mathrm{H}_{2}\right) \\
& -3316 / 27\left(1+9397 / 14922 / x-887 / 1658 x-6143 / 7461 x^{2}\right) \mathrm{H}_{1}-32(1+157 / 162 / x \\
& \left.-7 / 9 x-211 / 162 x^{2}\right) \mathrm{H}_{1,0}-8 / 9\left(1+4 / 3 / x-x-4 / 3 x^{2}\right)\left(7 \mathrm{H}_{1,0,0}+4 \mathrm{H}_{1,1,1}\right) \\
& -24\left(1+346 / 243 / x-7 / 9 x-400 / 243 x^{2}\right) \mathrm{H}_{1,1}-8 / 3\left(1+32 / 9 / x-1 / 3 x-20 / 9 x^{2}\right) \mathrm{H}_{1,1,0} \\
& +p_{\operatorname{gg}}(x)\left(-350 / 9+112 / 3 \zeta_{3}-100 / 9 \mathrm{H}_{0}+32 / 9 \mathrm{H}_{0} \zeta_{2}-20 / 3 \mathrm{H}_{1}+32 / 9 \mathrm{H}_{2,0}-32 / 9 \mathrm{H}_{3}\right) \\
& +(1+x)\left(40 / 3 \mathrm{H}_{0} \zeta_{3}+328 / 9 \mathrm{H}_{0,0} \zeta_{2}-536 / 9 \mathrm{H}_{0,0,0,0}+80 / 9 \mathrm{H}_{2} \zeta_{2}-112 / 9 \mathrm{H}_{2,0,0}\right. \\
& \left.-80 / 9 \mathrm{H}_{2,1,0}-64 / 9 \mathrm{H}_{2,1,1}-80 / 9 \mathrm{H}_{2,2}-208 / 9 \mathrm{H}_{3,0}-176 / 9 \mathrm{H}_{3,1}-328 / 9 \mathrm{H}_{4}\right) \\
& \left.+\delta(1-x)\left\{28945 / 162-1144 / 9 \zeta_{3}-100 / 9 \zeta_{2}-32 / 45 \zeta_{2}^{2}\right\}\right) \\
& +n_{f}^{3}\left(32 / 9\left(1-1 / 2 x+1 / 2 x^{2}\right)\left(25 / 18-\zeta_{2}+5 / 3 \mathrm{H}_{0}+\mathrm{H}_{0,0}+5 / 3 \mathrm{H}_{1}+\mathrm{H}_{1,0}+\mathrm{H}_{1,1}+\mathrm{H}_{2}\right)\right. \\
& +16 / 9 p_{\mathrm{gg}}(x)\left(25 / 18-\zeta_{2}+5 / 3 \mathrm{H}_{0}+\mathrm{H}_{0,0}+5 / 3 \mathrm{H}_{1}+\mathrm{H}_{1,0}+\mathrm{H}_{1,1}+\mathrm{H}_{2}\right) \\
& \left.+\delta(1-x)\left\{-1000 / 729+80 / 27 \zeta_{2}\right\}\right) \text {. }
\end{aligned}
$$

It may be worthwhile to note that, unlike the third-order coefficient functions for gauge-boson exchange DIS [9, 10], Eqs. (A.1) and (A.2) do not involve additional special functions including terms of the form $(1 \pm x)^{-n} \mathrm{H}_{\vec{w}}(x)$ with $n>1$. 


\section{Appendix B: The third-order physical kernels for $\left(F_{2}, F_{\phi}\right)$}

In this second appendix we write down the exact expressions, again using the notations introduced in Section 3, for the matrix elements of the NNLO contribution to the physical evolution kernel for the system $\left(F_{2}, F_{\phi}\right)$ of singlet structure functions. The coefficients in the expansion in powers of of the strong coupling are normalized according to Eq. (4.1).

As the corresponding NLO quantity before, we present $K_{22}^{(2)}$ via the decomposition (4.2) into its non-singlet and pure-singlet components. The former quantity, which is of direct interest for analyses of data on, e.g., the difference of the neutron and proton structure functions, is given by

$$
\begin{aligned}
& K_{2, \mathrm{~ns}}^{(2)}(x)= \\
& C_{A}^{2} C_{F}\left(p _ { \mathrm { qq } } ( x ) \left(50689 / 162-20 / 3 \zeta_{3}-1504 / 9 \zeta_{2}+192 / 5 \zeta_{2}^{2}+16 \mathrm{H}_{-3,0}+64 \mathrm{H}_{-2,-1,0}\right.\right. \\
& +64 \mathrm{H}_{-2,0}-16 \mathrm{H}_{-2,0,0}+32 \mathrm{H}_{-2,2}+10585 / 27 \mathrm{H}_{0}+64 \mathrm{H}_{0} \zeta_{3}-212 / 3 \mathrm{H}_{0} \zeta_{2} \\
& +1988 / 9 \mathrm{H}_{0,0}+256 / 3 \mathrm{H}_{0,0,0}-16 \mathrm{H}_{0,0,0,0}+4649 / 27 \mathrm{H}_{1}+144 \mathrm{H}_{1} \zeta_{3}-88 \mathrm{H}_{1} \zeta_{2} \\
& +96 \mathrm{H}_{1,-2,0}+484 / 9 \mathrm{H}_{1,0}-16 \mathrm{H}_{1,0} \zeta_{2}+44 / 3 \mathrm{H}_{1,0,0}-48 \mathrm{H}_{1,0,0,0}+484 / 9 \mathrm{H}_{1,1} \\
& \left.-88 / 3 \mathrm{H}_{1,1,0}-64 \mathrm{H}_{1,1,0,0}+88 / 3 \mathrm{H}_{1,2}+64 \mathrm{H}_{1,3}+968 / 9 \mathrm{H}_{2}-32 \mathrm{H}_{2,0,0}+44 \mathrm{H}_{3}+16 \mathrm{H}_{4}\right) \\
& +p_{\mathrm{qq}}(-x)\left(176 / 3 \zeta_{3}+536 / 9 \zeta_{2}-16 \zeta_{2}^{2}-16 \mathrm{H}_{-3,0}+128 \mathrm{H}_{-2} \zeta_{2}+352 / 3 \mathrm{H}_{-2,0}\right. \\
& -64 \mathrm{H}_{-2,0,0}-128 \mathrm{H}_{-2,2}+192 \mathrm{H}_{-1} \zeta_{3}-176 / 3 \mathrm{H}_{-1} \zeta_{2}-256 \mathrm{H}_{-1,-1} \zeta_{2}-352 / 3 \mathrm{H}_{-1,-1,0} \\
& +128 \mathrm{H}_{-1,-1,0,0}+256 \mathrm{H}_{-1,-1,2}+1072 / 9 \mathrm{H}_{-1,0}+176 \mathrm{H}_{-1,0} \zeta_{2}+176 \mathrm{H}_{-1,0,0} \\
& -48 \mathrm{H}_{-1,0,0,0}-128 \mathrm{H}_{-1,3}-124 / 3 \mathrm{H}_{0}-64 \mathrm{H}_{0} \zeta_{3}+80 / 3 \mathrm{H}_{0} \zeta_{2}-536 / 9 \mathrm{H}_{0,0}-48 \mathrm{H}_{0,0} \zeta_{2} \\
& \left.-256 / 3 \mathrm{H}_{0,0,0}+16 \mathrm{H}_{0,0,0,0}+32 \mathrm{H}_{2} \zeta_{2}+32 \mathrm{H}_{4}\right)+(1+x)\left(-96 \mathrm{H}_{-1,2}+112 \mathrm{H}_{2}-8 \mathrm{H}_{2} \zeta_{2}\right. \\
& \left.-4 \mathrm{H}_{2,0,0}+48 \mathrm{H}_{3}+12 \mathrm{H}_{4}\right)+(1-x)\left(8 \mathrm{H}_{-3,0}-8 \mathrm{H}_{-2} \zeta_{2}-16 \mathrm{H}_{-2,-1,0}+8 \mathrm{H}_{-2,0,0}\right) \\
& +16 / 3(1-43 x) \mathrm{H}_{-1,-1,0}+8 / 3(1+43 x)\left(\mathrm{H}_{1} \zeta_{2}-\mathrm{H}_{1,0,0}\right)-4 \zeta_{2}(3+5 x) \mathrm{H}_{0,0} \\
& +2 \zeta_{2}^{2}(5+3 x)-8 \zeta_{2}(6+11 x) \mathrm{H}_{0}+8 / 3(13-15 x) \mathrm{H}_{-2,0}-8 / 3(19-25 x) \mathrm{H}_{-1,0,0} \\
& -4 / 3 \zeta_{3}(27-91 x)+8 / 3 \zeta_{2}(37-7 x) \mathrm{H}_{-1}-8 / 15 \zeta_{2}\left(210-145 x+198 x^{3}\right) \\
& +11 / 9(229-467 x) \mathrm{H}_{1}+8 / 15\left(575+22 x^{-2}+355 x-198 x^{3}\right) \mathrm{H}_{-1,0}+1 / 45(2619 \\
& \left.-528 x^{-1}-33241 x+4752 x^{2}\right) \mathrm{H}_{0}+1 / 270\left(64993+3168 x^{-1}-283643 x+28512 x^{2}\right) \\
& -8 / 15\left(145 x-198 x^{3}\right) \mathrm{H}_{0,0}+x\left(-16 \mathrm{H}_{0} \zeta_{3}+40 \mathrm{H}_{0,0,0}+8 \mathrm{H}_{0,0,0,0}\right) \\
& \left.+\left(20 / 27\left(827+54 \zeta_{5}\right)-1544 / 3 \zeta_{3}+22286 / 27 \zeta_{2}-1592 / 15 \zeta_{2}^{2}\right) \delta(1-x)\right) \\
& +C_{A} C_{F}^{2}\left(p _ { \mathrm { qq } } ( x ) \left(11 / 2-664 / 3 \zeta_{3}-276 / 5 \zeta_{2}^{2}-16 \mathrm{H}_{-3,0}-48 \mathrm{H}_{-2} \zeta_{2}-224 \mathrm{H}_{-2,-1,0}\right.\right. \\
& -128 \mathrm{H}_{-2,0}+80 \mathrm{H}_{-2,0,0}-64 \mathrm{H}_{-2,2}-317 / 2 \mathrm{H}_{0}-136 \mathrm{H}_{0} \zeta_{3}-188 / 3 \mathrm{H}_{0} \zeta_{2}-74 \mathrm{H}_{0,0} \\
& -64 \mathrm{H}_{0,0} \zeta_{2}-4 / 3 \mathrm{H}_{0,0,0}+80 \mathrm{H}_{0,0,0,0}-384 \mathrm{H}_{1} \zeta_{3}+176 / 3 \mathrm{H}_{1} \zeta_{2}-256 \mathrm{H}_{1,-2,0} \\
& +1468 / 9 \mathrm{H}_{1,0}-32 \mathrm{H}_{1,0} \zeta_{2}+584 / 3 \mathrm{H}_{1,0,0}+176 \mathrm{H}_{1,0,0,0}+352 / 3 \mathrm{H}_{1,1,0}+128 \mathrm{H}_{1,1,0,0} \\
& +176 / 3 \mathrm{H}_{1,2}-128 \mathrm{H}_{1,3}+1072 / 9 \mathrm{H}_{2}-32 \mathrm{H}_{2} \zeta_{2}+440 / 3 \mathrm{H}_{2,0}+80 \mathrm{H}_{2,0,0}+88 \mathrm{H}_{2,1}
\end{aligned}
$$




$$
\begin{aligned}
& \left.+128 \mathrm{H}_{3}+16 \mathrm{H}_{3,0}+16 \mathrm{H}_{4}\right)+p_{\mathrm{qq}}(-x)\left(-244 / 3 \zeta_{3}-1072 / 9 \zeta_{2}+4 \zeta_{2}^{2}+80 \mathrm{H}_{-3,0}\right. \\
& -512 \mathrm{H}_{-2} \zeta_{2}-64 \mathrm{H}_{-2,-1,0}-776 / 3 \mathrm{H}_{-2,0}+336 \mathrm{H}_{-2,0,0}+480 \mathrm{H}_{-2,2}-672 \mathrm{H}_{-1} \zeta_{3} \\
& +208 / 3 \mathrm{H}_{-1} \zeta_{2}-64 \mathrm{H}_{-1,-2,0}+896 \mathrm{H}_{-1,-1} \zeta_{2}+704 / 3 \mathrm{H}_{-1,-1,0}-576 \mathrm{H}_{-1,-1,0,0} \\
& -896 \mathrm{H}_{-1,-1,2}-2144 / 9 \mathrm{H}_{-1,0}-672 \mathrm{H}_{-1,0} \zeta_{2}-376 \mathrm{H}_{-1,0,0}+272 \mathrm{H}_{-1,0,0,0}+48 \mathrm{H}_{-1,2} \\
& +32 \mathrm{H}_{-1,2,0}+512 \mathrm{H}_{-1,3}+284 / 3 \mathrm{H}_{0}+232 \mathrm{H}_{0} \zeta_{3}-124 / 3 \mathrm{H}_{0} \zeta_{2}+1072 / 9 \mathrm{H}_{0,0} \\
& \left.+208 \mathrm{H}_{0,0} \zeta_{2}+620 / 3 \mathrm{H}_{0,0,0}-80 \mathrm{H}_{0,0,0,0}-112 \mathrm{H}_{2} \zeta_{2}-24 \mathrm{H}_{3}-16 \mathrm{H}_{3,0}-160 \mathrm{H}_{4}\right) \\
& +(1+x)\left(384 \mathrm{H}_{-1,2}+16 \mathrm{H}_{2} \zeta_{2}-44 / 3 \mathrm{H}_{2,0}+32 \mathrm{H}_{2,0,0}-48 \mathrm{H}_{4}\right)+(1-x)\left(16 \mathrm{H}_{-2} \zeta_{2}\right. \\
& \left.+32 \mathrm{H}_{-2,-1,0}-48 \mathrm{H}_{-2,0,0}+224 / 3 \mathrm{H}_{1,0}\right)+16 / 3(1+43 x) \mathrm{H}_{1,0,0}-32(2-x) \mathrm{H}_{-3,0} \\
& -16 \zeta_{3}(3-2 x) \mathrm{H}_{0}+16 \zeta_{2}(3+5 x) \mathrm{H}_{0,0}-64 / 3(5-17 x) \mathrm{H}_{-1,-1,0}-32 / 3 \zeta_{2}(5+17 x) \mathrm{H}_{1} \\
& -8 \zeta_{2}^{2}(7+6 x)-44 / 3(7+25 x) \mathrm{H}_{0,0,0}-8 / 3(11-75 x) \mathrm{H}_{-2,0}-16(22+35 x) \mathrm{H}_{2} \\
& +32 / 3(29+7 x) \mathrm{H}_{-1,0,0}-32 / 3 \zeta_{2}(41+19 x) \mathrm{H}_{-1}-44 / 3(49-72 x) \mathrm{H}_{1} \\
& -8 / 3(65+101 x) \mathrm{H}_{3}+8 / 3 \zeta_{2}(65+176 x) \mathrm{H}_{0}+4 / 3 \zeta_{3}(99+61 x)-32 / 15(280 \\
& \left.+11 x^{-2}+170 x-99 x^{3}\right) \mathrm{H}_{-1,0}+16 / 15 \zeta_{2}\left(330+185 x+198 x^{3}\right)-2 / 45(635+7115 x \\
& \left.+4752 x^{3}\right) \mathrm{H}_{0,0}+1 / 90\left(799+2112 x^{-1}+89679 x-19008 x^{2}\right) \mathrm{H}_{0} \\
& -1 / 90\left(10817+2112 x^{-1}-29627 x+19008 x^{2}\right)-64 x \mathrm{H}_{0,0,0,0}+\left(2 / 3\left(47+180 \zeta_{5}\right)\right. \\
& \left.\left.+2296 / 3 \zeta_{3}-1235 / 3 \zeta_{2}+16 \zeta_{2} \zeta_{3}-856 / 15 \zeta_{2}^{2}\right) \delta(1-x)\right) \\
& +C_{F}^{3}\left(p _ { \mathrm { qq } } ( x ) \left(72 / 5 \zeta_{2}^{2}-32 \mathrm{H}_{-3,0}+96 \mathrm{H}_{-2} \zeta_{2}+192 \mathrm{H}_{-2,-1,0}-96 \mathrm{H}_{-2,0,0}-3 \mathrm{H}_{0}\right.\right. \\
& +16 \mathrm{H}_{0} \zeta_{3}-24 \mathrm{H}_{0} \zeta_{2}+26 \mathrm{H}_{0,0}-32 \mathrm{H}_{0,0,0,0}+192 \mathrm{H}_{1} \zeta_{3}+128 \mathrm{H}_{1,-2,0}-96 \mathrm{H}_{1,0,0} \\
& -64 \mathrm{H}_{1,0,0,0}+64 \mathrm{H}_{1,2,0}+128 \mathrm{H}_{1,3}-48 \mathrm{H}_{2,0}+32 \mathrm{H}_{2,0,0}+64 \mathrm{H}_{2,1,0}+64 \mathrm{H}_{2,2}+64 \mathrm{H}_{3,0} \\
& \left.+64 \mathrm{H}_{3,1}+32 \mathrm{H}_{4}\right)+p_{\mathrm{qq}}(-x)\left(-72 \zeta_{3}+56 \zeta_{2}^{2}-96 \mathrm{H}_{-3,0}+512 \mathrm{H}_{-2} \zeta_{2}+128 \mathrm{H}_{-2,-1,0}\right. \\
& +48 \mathrm{H}_{-2,0}-416 \mathrm{H}_{-2,0,0}-448 \mathrm{H}_{-2,2}+576 \mathrm{H}_{-1} \zeta_{3}+96 \mathrm{H}_{-1} \zeta_{2}+128 \mathrm{H}_{-1,-2,0} \\
& -768 \mathrm{H}_{-1,-1} \zeta_{2}+640 \mathrm{H}_{-1,-1,0,0}+768 \mathrm{H}_{-1,-1,2}+640 \mathrm{H}_{-1,0} \zeta_{2}+48 \mathrm{H}_{-1,0,0} \\
& -352 \mathrm{H}_{-1,0,0,0}-96 \mathrm{H}_{-1,2}-64 \mathrm{H}_{-1,2,0}-512 \mathrm{H}_{-1,3}-24 \mathrm{H}_{0}-208 \mathrm{H}_{0} \zeta_{3}-24 \mathrm{H}_{0} \zeta_{2} \\
& \left.-224 \mathrm{H}_{0,0} \zeta_{2}-72 \mathrm{H}_{0,0,0}+96 \mathrm{H}_{0,0,0,0}+96 \mathrm{H}_{2} \zeta_{2}+48 \mathrm{H}_{3}+32 \mathrm{H}_{3,0}+192 \mathrm{H}_{4}\right) \\
& +(1+x)\left(296 / 5 \zeta_{2}^{2}+480 \mathrm{H}_{-1} \zeta_{2}+192 \mathrm{H}_{-1,-1,0}-32 \mathrm{H}_{-1,0}-416 \mathrm{H}_{-1,0,0}-384 \mathrm{H}_{-1,2}\right. \\
& \left.-48 \mathrm{H}_{2,0,0}-32 \mathrm{H}_{3,0}-16 \mathrm{H}_{4}\right)+(1-x)\left(-62+64 \mathrm{H}_{-2,0,0}+16 \mathrm{H}_{0,0} \zeta_{2}-48 \mathrm{H}_{0,0,0,0}\right. \\
& \left.+560 \mathrm{H}_{1}+96 \mathrm{H}_{1} \zeta_{2}-16 \mathrm{H}_{1,0}\right)-80(1+3 x) \mathrm{H}_{-2,0}+8(3-5 x) \mathrm{H}_{2,0}+32(3-x) \mathrm{H}_{-3,0} \\
& +16(3+20 x) \mathrm{H}_{0,0,0}-(3+539 x) \mathrm{H}_{0}-24 \zeta_{3}(5+27 x)-2(9-191 x) \mathrm{H}_{0,0} \\
& +16(9+13 x) \mathrm{H}_{3}-16 \zeta_{2}(9+28 x) \mathrm{H}_{0}+4(77+85 x) \mathrm{H}_{2}-4 \zeta_{2}(77+93 x)+96 \zeta_{3} \mathrm{H}_{0} \\
& \left.+\left(1 / 2\left(29-480 \zeta_{5}\right)+68 \zeta_{3}+18 \zeta_{2}-32 \zeta_{2} \zeta_{3}+288 / 5 \zeta_{2}^{2}\right) \delta(1-x)\right) \\
& +C_{A} C_{F} n_{f}\left(p _ { \mathrm { qq } } ( x ) \left(-7531 / 81-64 / 3 \zeta_{3}+48 \zeta_{2}-16 \mathrm{H}_{-2,0}-3536 / 27 \mathrm{H}_{0}+32 / 3 \mathrm{H}_{0} \zeta_{2}\right.\right. \\
& -608 / 9 \mathrm{H}_{0,0}-40 / 3 \mathrm{H}_{0,0,0}-1552 / 27 \mathrm{H}_{1}+16 \mathrm{H}_{1} \zeta_{2}-176 / 9 \mathrm{H}_{1,0}-8 / 3 \mathrm{H}_{1,0,0}
\end{aligned}
$$




$$
\begin{aligned}
& \left.-176 / 9 \mathrm{H}_{1,1}+16 / 3 \mathrm{H}_{1,1,0}-16 / 3 \mathrm{H}_{1,2}-352 / 9 \mathrm{H}_{2}-8 \mathrm{H}_{3}\right) \\
& +p_{\mathrm{qq}}(-x)\left(-32 / 3 \zeta_{3}-80 / 9 \zeta_{2}-64 / 3 \mathrm{H}_{-2,0}+32 / 3 \mathrm{H}_{-1} \zeta_{2}+64 / 3 \mathrm{H}_{-1,-1,0}\right. \\
& \left.-160 / 9 \mathrm{H}_{-1,0}-32 \mathrm{H}_{-1,0,0}+16 / 3 \mathrm{H}_{0}-8 / 3 \mathrm{H}_{0} \zeta_{2}+80 / 9 \mathrm{H}_{0,0}+40 / 3 \mathrm{H}_{0,0,0}\right) \\
& -8(1+x) \mathrm{H}_{2}+16 / 3(1-5 x)\left(\mathrm{H}_{1} \zeta_{2}-\mathrm{H}_{1,0,0}\right)+16 / 3(1+5 x)\left(\mathrm{H}_{-1} \zeta_{2}+2 \mathrm{H}_{-1,-1,0}\right. \\
& \left.-\mathrm{H}_{-1,0,0}\right)-16 / 45\left(3-6 x^{-1}-497 x+54 x^{2}\right) \mathrm{H}_{0}+8 / 15 \zeta_{2}\left(15-55 x+36 x^{3}\right) \\
& -16 / 15\left(55+2 x^{-2}+35 x-18 x^{3}\right) \mathrm{H}_{-1,0}-4 / 9(73-269 x) \mathrm{H}_{1}-1 / 135\left(7303+288 x^{-1}\right. \\
& \left.-43013 x+2592 x^{2}\right)+8 / 15\left(55 x-36 x^{3}\right) \mathrm{H}_{0,0}-160 / 3 \zeta_{3} x-32 / 3 \mathrm{H}_{-2,0}-(5516 / 27 \\
& \left.\left.-224 / 3 \zeta_{3}+7216 / 27 \zeta_{2}-296 / 15 \zeta_{2}^{2}\right) \delta(1-x)\right) \\
& +C_{F}^{2} n_{f}\left(p _ { \mathrm { qq } } ( x ) \left(-67 / 3+208 / 3 \zeta_{3}+32 \mathrm{H}_{-2,0}+19 \mathrm{H}_{0}+80 / 3 \mathrm{H}_{0} \zeta_{2}+12 \mathrm{H}_{0,0}\right.\right. \\
& -32 / 3 \mathrm{H}_{0,0,0}-4 \mathrm{H}_{1}-32 / 3 \mathrm{H}_{1} \zeta_{2}-232 / 9 \mathrm{H}_{1,0}-80 / 3 \mathrm{H}_{1,0,0}-64 / 3 \mathrm{H}_{1,1,0}-32 / 3 \mathrm{H}_{1,2} \\
& \left.-160 / 9 \mathrm{H}_{2}-80 / 3 \mathrm{H}_{2,0}-16 \mathrm{H}_{2,1}-32 \mathrm{H}_{3}\right)+p_{\mathrm{qq}}(-x)\left(64 / 3 \zeta_{3}+160 / 9 \zeta_{2}\right. \\
& +128 / 3 \mathrm{H}_{-2,0}-64 / 3 \mathrm{H}_{-1} \zeta_{2}-128 / 3 \mathrm{H}_{-1,-1,0}+320 / 9 \mathrm{H}_{-1,0}+64 \mathrm{H}_{-1,0,0}-32 / 3 \mathrm{H}_{0} \\
& \left.+16 / 3 \mathrm{H}_{0} \zeta_{2}-160 / 9 \mathrm{H}_{0,0}-80 / 3 \mathrm{H}_{0,0,0}\right)+8 / 3(1+x)\left(-2 \mathrm{H}_{0} \zeta_{2}+7 \mathrm{H}_{0,0,0}+\mathrm{H}_{2,0}\right. \\
& \left.+2 \mathrm{H}_{3}\right)-32 / 3(1-x) \mathrm{H}_{1,0}-32 / 3(1-5 x)\left(\mathrm{H}_{1} \zeta_{2}-\mathrm{H}_{1,0,0}\right)+16(1+3 x) \mathrm{H}_{2} \\
& -32 / 3(1+5 x)\left(\mathrm{H}_{-1} \zeta_{2}+2 \mathrm{H}_{-1,-1,0}-\mathrm{H}_{-1,0,0}\right)-16 / 15 \zeta_{2}\left(15-25 x+36 x^{3}\right) \\
& +8 / 3(19-42 x) \mathrm{H}_{1}+32 / 15\left(55+2 x^{-2}+35 x-18 x^{3}\right) \mathrm{H}_{-1,0}+4 / 45(85-95 x \\
& \left.+432 x^{3}\right) \mathrm{H}_{0,0}+8 / 45\left(134+24 x^{-1}-269 x+216 x^{2}\right)-1 / 45\left(209+192 x^{-1}+4929 x\right. \\
& \left.-1728 x^{2}\right) \mathrm{H}_{0}+320 / 3 \zeta_{3} x+64 / 3 \mathrm{H}_{-2,0}-\left(239 / 6+400 / 3 \zeta_{3}-146 / 3 \zeta_{2}\right. \\
& \left.\left.-208 / 15 \zeta_{2}^{2}\right) \delta(1-x)\right) \\
& +C_{F} n_{f}^{2}\left(p _ { \mathrm { qq } } ( x ) \left(470 / 81-32 / 9 \zeta_{2}+268 / 27 \mathrm{H}_{0}+16 / 3 \mathrm{H}_{0,0}+116 / 27 \mathrm{H}_{1}+16 / 9 \mathrm{H}_{1,0}\right.\right. \\
& \left.+16 / 9 \mathrm{H}_{1,1}+32 / 9 \mathrm{H}_{2}\right)-4 / 9(1+13 x) \mathrm{H}_{1}-4 / 9(3+23 x) \mathrm{H}_{0}+2 / 27(29-295 x) \\
& \left.+\left(406 / 27+536 / 27 \zeta_{2}\right) \delta(1-x)\right) \text {. }
\end{aligned}
$$

The corresponding pure-singlet component reads

$$
\begin{aligned}
& K_{22, \mathrm{ps}}^{(2)}(x)= \\
& C_{A} C_{F} n_{f}\left(8(1+x)\left(4 \mathrm{H}_{2} \zeta_{2}-7 \mathrm{H}_{2,0,0}-4 \mathrm{H}_{3,0}-\mathrm{H}_{4}\right)+8(1-x)\left(4 \mathrm{H}_{-2} \zeta_{2}+8 \mathrm{H}_{-2,-1,0}\right.\right. \\
& \left.\quad+4 \mathrm{H}_{-2,0,0}+\mathrm{H}_{0,0} \zeta_{2}\right)+32 \zeta_{3}(2+x) \mathrm{H}_{0}+64 / 3\left(3-x^{-1}+3 x-x^{2}\right) \mathrm{H}_{-1,-1,0} \\
& \quad+32 / 3 \zeta_{2}\left(3+x^{-1}-3 x-x^{2}\right) \mathrm{H}_{1}-32 / 3\left(3+2 x^{-1}+3 x+2 x^{2}\right) \mathrm{H}_{-1,2}-16 / 3\left(3+8 x^{-1}\right. \\
& \left.\quad+3 x+8 x^{2}\right) \mathrm{H}_{-1,0,0}+16(3-x) \mathrm{H}_{-3,0}+16(4-3 x) \mathrm{H}_{0,0,0,0}+8 / 3\left(5-13 x+8 x^{2}\right) \mathrm{H}_{2,0} \\
& \quad+32 / 3 \zeta_{2}\left(6+x^{-1}+6 x+x^{2}\right) \mathrm{H}_{-1}-8 / 3 \zeta_{3}\left(11-32 x^{-1}+89 x+28 x^{2}\right) \\
& \quad+4 / 5 \zeta_{2}^{2}(11+31 x)-4 / 3 \zeta_{2}\left(15-16 x^{-1}+15 x+16 x^{2}\right) \mathrm{H}_{0}+4 / 3\left(15+16 x^{-1}-51 x\right. \\
& \left.\quad+16 x^{2}\right) \mathrm{H}_{3}-8(15+32 x) \mathrm{H}_{0,0,0}-4 / 9 \zeta_{2}\left(21-368 x^{-1}-363 x+160 x^{2}\right) \\
& \quad+4 / 9\left(21+104 x^{-1}+21 x+160 x^{2}\right) \mathrm{H}_{2}-8 / 3\left(27-16 x^{-1}+33 x+24 x^{2}\right) \mathrm{H}_{-2,0}
\end{aligned}
$$




$$
\begin{aligned}
& -4 / 3\left(39+16 x^{-1}-39 x-16 x^{2}\right) \mathrm{H}_{1,0,0}-4 / 3\left(49-32 x^{-1}-x-16 x^{2}\right) \mathrm{H}_{1,0} \\
& -4 / 9\left(57-127 x^{-1}-48 x+118 x^{2}\right) \mathrm{H}_{1}+32 / 9\left(84+59 x^{-1}+48 x+23 x^{2}\right) \mathrm{H}_{-1,0} \\
& +4 / 9\left(743+28 x^{-1}-874 x+744 x^{2}\right) \mathrm{H}_{0,0}+1 / 27\left(8115-15971 x^{-1}+8688 x-832 x^{2}\right) \\
& \left.-2 / 27\left(11373+820 x^{-1}+1860 x-1396 x^{2}\right) \mathrm{H}_{0}\right) \\
+ & C_{F}^{2} n_{f}\left(1 6 ( 1 + x ) \left(-3 \mathrm{H}_{-1} \zeta_{2}-6 \mathrm{H}_{-1,-1,0}+3 \mathrm{H}_{-1,0,0}+6 \mathrm{H}_{0,0} \zeta_{2}-4 \mathrm{H}_{0,0,0,0}-2 \mathrm{H}_{2} \zeta_{2}\right.\right. \\
& \left.+2 \mathrm{H}_{2,0,0}-2 \mathrm{H}_{3,0}-6 \mathrm{H}_{4}\right)+16(1-x)\left(-2 \mathrm{H}_{-2} \zeta_{2}-4 \mathrm{H}_{-2,-1,0}+2 \mathrm{H}_{-2,0,0}+2 \mathrm{H}_{0} \zeta_{3}\right. \\
& \left.-3 \mathrm{H}_{1} \zeta_{2}+3 \mathrm{H}_{1,0,0}\right)-16 / 3\left(3-4 x^{-1}-8 x^{2}\right) \mathrm{H}_{2,0}+16 / 3 \zeta_{3}\left(3+4 x^{-1}+36 x\right) \\
& +16 / 3\left(3-7 x+24 x^{2}\right) \mathrm{H}_{0,0,0}-16 / 3\left(9-4 x^{-1}+12 x-16 x^{2}\right) \mathrm{H}_{3}+16 / 3 \zeta_{2}\left(9-4 x^{-1}\right. \\
& \left.+16 x-16 x^{2}\right) \mathrm{H}_{0}+32 / 3(9+2 x) \mathrm{H}_{-2,0}+16 / 5 \zeta_{2}^{2}(11+6 x)-32 / 3\left(12-5 x^{-1}-12 x\right. \\
+ & \left.5 x^{2}\right) \mathrm{H}_{1,0}-8 / 9\left(234-80 x^{-1}-27 x+32 x^{2}\right) \mathrm{H}_{2}+16 / 45\left(585+4 x^{-2}+625 x\right. \\
& \left.-36 x^{3}\right) \mathrm{H}_{-1,0}-4 / 9\left(657-149 x^{-1}-450 x-58 x^{2}\right) \mathrm{H}_{1}+8 / 45 \zeta_{2}\left(1170-400 x^{-1}\right. \\
+ & \left.1115 x+160 x^{2}-72 x^{3}\right)-2 / 45\left(1484-628 x^{-1}-7911 x-1588 x^{2}\right) \mathrm{H}_{0} \\
& -4 / 45\left(3015+1825 x+920 x^{2}-144 x^{3}\right) \mathrm{H}_{0,0}-1 / 45\left(6931-5184 x^{-1}-7391 x\right. \\
+ & \left.\left.5644 x^{2}\right)+64 \mathrm{H}_{-3,0}\right) \\
+ & C_{F} n_{f}^{2}\left(16 / 3(1+x)\left(-2 \zeta_{3}+3 \mathrm{H}_{0,0,0}-\mathrm{H}_{2,0}\right)-64 / 9\left(3+x^{-1}+3 x+x^{2}\right) \mathrm{H}_{-1,0}\right. \\
+ & 8 / 9\left(3+4 x^{-1}-3 x-4 x^{2}\right) \mathrm{H}_{1,0}+8 / 9\left(23-12 x^{-1}+95 x-20 x^{2}\right) \mathrm{H}_{0,0} \\
+ & 4 / 27\left(351-212 x^{-1}+114 x-240 x^{2}\right) \mathrm{H}_{0}+2 / 81\left(5139-2155 x^{-1}-3420 x+436 x^{2}\right) \\
& \left.-64 / 9 \zeta_{2}\left(x^{-1}+3 x\right)\right) .
\end{aligned}
$$

The off-diagonal contributions $K_{\mathrm{qg}}^{(2)}$ and $K_{\mathrm{gq}}^{(2)}$ can be written as

$$
\begin{aligned}
& K_{2 \phi}^{(2)}(x)= \\
& C_{A}^{2} n_{f}\left(8 p _ { \mathrm { qg } } ( x ) \left[-11 \zeta_{3} \mathrm{H}_{1}-8 \zeta_{2} \mathrm{H}_{1,0}+4 \mathrm{H}_{1,0,0,0}-8 \zeta_{2} \mathrm{H}_{1,1}+12 \mathrm{H}_{1,1,0}+4 \mathrm{H}_{1,1,0,0}\right.\right. \\
&\left.\quad+14 / 3 \mathrm{H}_{1,2}+4 \mathrm{H}_{1,3}+61 / 3 \mathrm{H}_{2,1}+4 \mathrm{H}_{2,1,0}+4 \mathrm{H}_{2,2}+8 \mathrm{H}_{3,1}\right]+4 p_{\mathrm{qg}}(-x)\left[-16 \mathrm{H}_{-2,2}\right. \\
&-6 \zeta_{3} \mathrm{H}_{-1}-16 \mathrm{H}_{-1,-2,0}+8 \zeta_{2} \mathrm{H}_{-1,-1}+32 \mathrm{H}_{-1,-1,-1,0}+4 \mathrm{H}_{-1,-1,0,0}+12 \zeta_{2} \mathrm{H}_{-1,0} \\
&\left.-8 \mathrm{H}_{-1,0,0,0}-20 \mathrm{H}_{-1,3}\right]+8 / 3 \zeta_{2}\left(-16 x^{-1}+1+11 x+18 x^{2}\right) \mathrm{H}_{-1} \\
&+32\left(1-4 x-6 x^{2}\right) \mathrm{H}_{-1,2}+8(1-2 x)\left[-7 \zeta_{3} \mathrm{H}_{1}-4 \zeta_{2} \mathrm{H}_{1,0}+2 \mathrm{H}_{1,0,0,0}-4 \zeta_{2} \mathrm{H}_{1,1}\right. \\
&\left.+2 \mathrm{H}_{1,1,0,0}+2 \mathrm{H}_{1,3}\right]+8(1+2 x)\left[4 \mathrm{H}_{-2,2}-7 \zeta_{3} \mathrm{H}_{-1}-4 \mathrm{H}_{-1,-2,0}+8 \zeta_{2} \mathrm{H}_{-1,-1}\right. \\
&\left.+8 \mathrm{H}_{-1,-1,-1,0}-6 \mathrm{H}_{-1,-1,0,0}-4 \zeta_{2} \mathrm{H}_{-1,0}+2 \mathrm{H}_{-1,0,0,0}+2 \mathrm{H}_{-1,3}\right]-8 / 3(1+345 x \\
&\left.-36 x^{2}\right) \mathrm{H}_{0,0,0}+64 \zeta_{3}(2+9 x) \mathrm{H}_{0}-8(3+2 x) \mathrm{H}_{-3,0}-12\left(3+14 x-8 x^{2}\right) \mathrm{H}_{4} \\
&+16(4-15 x) \mathrm{H}_{0,0,0,0}-8\left(5+6 x+16 x^{2}\right) \mathrm{H}_{-2,0,0}+8 \zeta_{2}(7+38 x) \mathrm{H}_{2}+4 \zeta_{2}(9 \\
&\left.+38 x-24 x^{2}\right) \mathrm{H}_{0,0}+16\left(11-30 x+8 x^{2}\right) \mathrm{H}_{-2,-1,0}+16 / 3\left(-16 x^{-1}+13-37 x\right. \\
&\left.-54 x^{2}\right) \mathrm{H}_{-1,-1,0}-8 / 3\left(-8 x^{-1}+15+420 x-436 x^{2}\right) \mathrm{H}_{1}+8 \zeta_{2}(15-22 x \\
&\left.+24 x^{2}\right) \mathrm{H}_{-2}+4 / 3\left(32 x^{-1}+17-691 x+308 x^{2}\right) \mathrm{H}_{3}-4 / 3 \zeta_{2}\left(17-441 x+308 x^{2}\right) \mathrm{H}_{0}
\end{aligned}
$$




$$
\begin{aligned}
& +8 / 3\left(17-184 x+116 x^{2}\right) \mathrm{H}_{2,0}-8 / 3\left(17+127 x+150 x^{2}\right) \mathrm{H}_{-1,0,0}+8 / 3 \zeta_{2}\left(16 x^{-1}\right. \\
& \left.+23+65 x-82 x^{2}\right) \mathrm{H}_{1}-4\left(25+90 x+8 x^{2}\right) \mathrm{H}_{2,0,0}+8 / 3\left(37-222 x+210 x^{2}\right) \mathrm{H}_{1,1} \\
& -8 / 3\left(-16 x^{-1}+46+125 x-10 x^{2}\right) \mathrm{H}_{-2,0}-4 / 3\left(32 x^{-1}+99+159 x-280 x^{2}\right) \mathrm{H}_{1,0,0} \\
& +8 / 9\left(244 x^{-1}+99-264 x-179 x^{2}\right) \mathrm{H}_{-1,0}+4 / 3 \zeta_{3}\left(80 x^{-1}+133+134 x-286 x^{2}\right) \\
& +2 / 5 \zeta_{2}^{2}\left(149+174 x+352 x^{2}\right)-2 / 9\left(-192 x^{-1}+163+2758 x-2824 x^{2}\right) \mathrm{H}_{1,0} \\
& -4 / 9\left(-200 x^{-1}+375+2514 x-1852 x^{2}\right) \mathrm{H}_{2}+4 / 9 \zeta_{2}\left(288 x^{-1}+375+1986 x\right. \\
& \left.-1852 x^{2}\right)-2 / 9\left(372 x^{-1}+1589+6362 x-2308 x^{2}\right) \mathrm{H}_{0}+2 / 9\left(56 x^{-1}+2039\right. \\
& \left.-4942 x+6340 x^{2}\right) \mathrm{H}_{0,0}+1 / 27\left(-19297 x^{-1}+25545-58161 x+46270 x^{2}\right) \\
& \left.-64\left(\left(3 x-x^{2}\right) \mathrm{H}_{3,0}-x^{2} \mathrm{H}_{-1,-1,2}+\mathrm{H}_{1,1,0}+\mathrm{H}_{1,2}+2 \mathrm{H}_{2,1}\right)\right) \\
& +C_{F}^{2} n_{f}\left(1 6 p _ { \mathrm { qg } } ( x ) \left[-4 \zeta_{2} \mathrm{H}_{0,0}-9 \zeta_{3} \mathrm{H}_{1}-8 \mathrm{H}_{1,-2,0}-10 \zeta_{2} \mathrm{H}_{1,0}+4 \mathrm{H}_{1,0,0,0}-4 \zeta_{2} \mathrm{H}_{1,1}\right.\right. \\
& \left.-3 \mathrm{H}_{1,1,0}+2 \mathrm{H}_{1,1,0,0}-3 \mathrm{H}_{1,2}+4 \mathrm{H}_{1,2,0}+6 \mathrm{H}_{1,3}-4 \zeta_{2} \mathrm{H}_{2}+4 \mathrm{H}_{3,0}+4 \mathrm{H}_{4}\right] \\
& +16 p_{\mathrm{gg}}(-x)\left[-8 \zeta_{2} \mathrm{H}_{-2}-8 \mathrm{H}_{-2,-1,0}+4 \mathrm{H}_{-2,0,0}-7 \zeta_{3} \mathrm{H}_{-1}+8 \zeta_{2} \mathrm{H}_{-1,-1}\right. \\
& \left.+8 \mathrm{H}_{-1,-1,-1,0}+2 \mathrm{H}_{-1,-1,0,0}-4 \mathrm{H}_{-1,-1,2}-6 \zeta_{2} \mathrm{H}_{-1,0}+2 \mathrm{H}_{-1,3}+4 \mathrm{H}_{0,0,0,0}\right] \\
& +16(1-2 x)\left[-7 \zeta_{3} \mathrm{H}_{1}-4 \zeta_{2} \mathrm{H}_{1,0}+2 \mathrm{H}_{1,0,0,0}-4 \zeta_{2} \mathrm{H}_{1,1}+2 \mathrm{H}_{1,1,0,0}+2 \mathrm{H}_{1,3}-2 \mathrm{H}_{3,0}\right. \\
& \left.-2 \mathrm{H}_{4}\right]+16(1+2 x)\left[-7 \zeta_{3} \mathrm{H}_{-1}-4 \mathrm{H}_{-1,-2,0}+8 \zeta_{2} \mathrm{H}_{-1,-1}+8 \mathrm{H}_{-1,-1,-1,0}\right. \\
& \left.-6 \mathrm{H}_{-1,-1,0,0}-4 \mathrm{H}_{-1,-1,2}-4 \zeta_{2} \mathrm{H}_{-1,0}+2 \mathrm{H}_{-1,0,0,0}+2 \mathrm{H}_{-1,3}-3 \mathrm{H}_{0,0,0,0}\right] \\
& -16 \zeta_{3}\left(1-34 x+4 x^{2}\right) \mathrm{H}_{0}+128\left(1+x^{2}\right) \mathrm{H}_{-2,2}+8\left(3-2 x-12 x^{2}\right) \mathrm{H}_{2,0}-32(3 \\
& \left.-2 x+8 x^{2}\right) \mathrm{H}_{-3,0}-32 / 5 \zeta_{2}\left(5-80 x-15 x^{2}-24 x^{3}\right) \mathrm{H}_{0}-8 / 5 \zeta_{2}^{2}\left(5+90 x-24 x^{2}\right) \\
& -16\left(7+2 x-12 x^{2}\right) \mathrm{H}_{1,0,0}+8\left(13-42 x+34 x^{2}\right) \mathrm{H}_{1,1} \\
& +16 / 15 \zeta_{2}\left(-x^{-2}+15+70 x-180 x^{2}+36 x^{3}\right) \mathrm{H}_{1}-16 / 3 \zeta_{3}\left(15-175 x-36 x^{2}\right. \\
& \left.-36 x^{3}\right)+32 / 15\left(15-55 x-45 x^{2}-36 x^{3}\right) \mathrm{H}_{3}+4\left(19-68 x+52 x^{2}\right) \mathrm{H}_{1,0} \\
& +32 / 15\left(-x^{-2}+30-160 x-270 x^{2}-36 x^{3}\right) \mathrm{H}_{-1,-1,0}-32 / 15\left(-x^{-2}+30+50 x\right. \\
& \left.-45 x^{2}-36 x^{3}\right) \mathrm{H}_{-1,0,0}-16 / 5 \zeta_{2}\left(x^{-2}+50+80 x+90 x^{2}+36 x^{3}\right) \mathrm{H}_{-1} \\
& +32 / 15\left(x^{-2}+90+40 x+36 x^{3}\right) \mathrm{H}_{-1,2}-32 / 15\left(150-185 x-270 x^{2}-36 x^{3}\right) \mathrm{H}_{-2,0} \\
& -4 / 15\left(8 x^{-1}+161+1986 x-732 x^{2}\right) \mathrm{H}_{2}-1 / 45\left(64 x^{-1}+217+3528 x\right. \\
& \left.-13320 x^{2}\right) \mathrm{H}_{0}+2 / 45\left(-48 x^{-1}+249+2494 x-1728 x^{2}\right. \\
& \left.-2880 x^{3}\right) \mathrm{H}_{0,0}+4 / 45 \zeta_{2}\left(48 x^{-1}+483+1426 x-2196 x^{2}+1440 x^{3}\right) \\
& -2 / 15\left(-16 x^{-1}+1849+756 x-2604 x^{2}\right) \mathrm{H}_{1}-1 / 45\left(-64 x^{-1}+2572+13953 x\right. \\
& \left.-16596 x^{2}\right)-8 / 45\left(-20 x^{-2}-12 x^{-1}+3651+2266 x-1602 x^{2}-720 x^{3}\right) \mathrm{H}_{-1,0} \\
& -64\left(4 x-x^{2}\right) \mathrm{H}_{2,0,0}-16 / 15\left(85 x-90 x^{2}+72 x^{3}\right) \mathrm{H}_{0,0,0}-192 x^{2} \mathrm{H}_{-2,0,0} \\
& \left.-32\left(2 \zeta_{2} \mathrm{H}_{-2}-\zeta_{2} \mathrm{H}_{0,0}-2 \mathrm{H}_{1,1,0}-2 \mathrm{H}_{1,2}\right)+128 x\left[-2 \mathrm{H}_{-2,-1,0}+\zeta_{2} \mathrm{H}_{2}\right]\right) \\
& +C_{A} C_{F} n_{f}\left(8 p _ { \mathrm { qg } } ( x ) \left[23 \zeta_{3} \mathrm{H}_{1}+12 \mathrm{H}_{1,-2,0}+22 \zeta_{2} \mathrm{H}_{1,0}-4 \mathrm{H}_{1,0,0,0}+16 \zeta_{2} \mathrm{H}_{1,1}\right.\right.
\end{aligned}
$$


$\left.+7 / 3 \mathrm{H}_{1,1,0}+29 / 3 \mathrm{H}_{1,2}-8 \mathrm{H}_{1,3}-12 \mathrm{H}_{2,1}+4 \mathrm{H}_{2,1,0}+4 \mathrm{H}_{2,2}\right]$

$+16 p_{\mathrm{qg}}(-x)\left[13 \zeta_{2} \mathrm{H}_{-2}+8 \mathrm{H}_{-1,-2,0}+9 \mathrm{H}_{-1,-1,0,0}+10 \mathrm{H}_{-1,-1,2}+12 \zeta_{2} \mathrm{H}_{-1,0}\right.$

$\left.-6 \mathrm{H}_{-1,0,0,0}-2 \mathrm{H}_{-1,2,0}-5 \mathrm{H}_{-1,3}-4 \zeta_{3} \mathrm{H}_{0}\right]+24(1-2 x)\left[7 \zeta_{3} \mathrm{H}_{1}+4 \zeta_{2} \mathrm{H}_{1,0}\right.$

$\left.-2 \mathrm{H}_{1,0,0,0}+4 \zeta_{2} \mathrm{H}_{1,1}-2 \mathrm{H}_{1,1,0,0}-2 \mathrm{H}_{1,3}\right]+16(1+2 x)\left[3 \zeta_{2} \mathrm{H}_{-2}+6 \mathrm{H}_{-1,-2,0}\right.$

$\left.+9 \mathrm{H}_{-1,-1,0,0}+6 \mathrm{H}_{-1,-1,2}+6 \zeta_{2} \mathrm{H}_{-1,0}-3 \mathrm{H}_{-1,0,0,0}-3 \mathrm{H}_{-1,3}-2 \mathrm{H}_{3,0}\right]$

$-32(1+3 x) \mathrm{H}_{0,0,0,0}+64\left(1+14 x+5 x^{2}\right) \mathrm{H}_{-2,-1,0}+8 \zeta_{2}\left(3-2 x-4 x^{2}\right) \mathrm{H}_{0,0}$

$-8\left(3+10 x-4 x^{2}\right) \mathrm{H}_{4}-96 \zeta_{2}\left(5+10 x+6 x^{2}\right) \mathrm{H}_{-1,-1}+4 / 15\left(5+1660 x+840 x^{2}\right.$

$\left.+288 x^{3}\right) \mathrm{H}_{3}-4 / 15 \zeta_{2}\left(5+2960 x+840 x^{2}+576 x^{3}\right) \mathrm{H}_{0}-32\left(7+2 x+8 x^{2}\right) \mathrm{H}_{-2,2}$

$-64\left(7+14 x+8 x^{2}\right) \mathrm{H}_{-1,-1,-1,0}-16\left(7+26 x+10 x^{2}\right) \mathrm{H}_{-2,0,0}+16(13-6 x$

$\left.+8 x^{2}\right) \mathrm{H}_{-3,0}+8\left(17+38 x+16 x^{2}\right) \mathrm{H}_{2,0,0}+16 \zeta_{3}\left(25+50 x+29 x^{2}\right) \mathrm{H}_{-1}$

$-4 / 3\left(-16 x^{-1}+33-42 x-60 x^{2}\right) \mathrm{H}_{2,0}-8 / 15 \zeta_{2}\left(-2 x^{-2}+60 x^{-1}+35+370 x\right.$

$\left.-465 x^{2}+72 x^{3}\right) \mathrm{H}_{1}+4 / 5 \zeta_{2}^{2}\left(59+314 x-120 x^{2}\right)+16 / 15\left(2 x^{-2}+60 x^{-1}+110\right.$

$\left.+660 x+755 x^{2}+72 x^{3}\right) \mathrm{H}_{-1,-1,0}-4 / 3\left(131-654 x+582 x^{2}\right) \mathrm{H}_{1,1}-4 / 3 \zeta_{3}(175$

$\left.+934 x+114 x^{2}+144 x^{3}\right)+4 / 15\left(195+550 x+1800 x^{2}+288 x^{3}\right) \mathrm{H}_{0,0,0}$

$-16 / 15\left(2 x^{-2}+40 x^{-1}+205+355 x+320 x^{2}+72 x^{3}\right) \mathrm{H}_{-1,0,0}$

$+4 / 3\left(16 x^{-1}+235-116 x-72 x^{2}\right) \mathrm{H}_{1,0,0}+4 / 15\left(128 x^{-1}+261+3881 x\right.$

$\left.-2142 x^{2}\right) \mathrm{H}_{2}+16 / 15\left(265-325 x-755 x^{2}-72 x^{3}\right) \mathrm{H}_{-2,0}+2 / 15\left(784 x^{-1}+399\right.$

$\left.+7156 x-8064 x^{2}\right) \mathrm{H}_{1}-16 / 15\left(2 x^{-2}+20 x^{-1}+420+350 x+65 x^{2}+72 x^{3}\right) \mathrm{H}_{-1,2}$

$-1 / 45\left(-848 x^{-1}+695-25786 x+30216 x^{2}\right) \mathrm{H}_{0}-4 / 45 \zeta_{2}\left(408 x^{-1}+783\right.$

$\left.+2531 x-6426 x^{2}+2016 x^{3}\right)-2 / 9\left(-256 x^{-1}+831-3264 x+2596 x^{2}\right) \mathrm{H}_{1,0}$

$-4 / 45\left(-24 x^{-1}+927+1897 x+4726 x^{2}-2016 x^{3}\right) \mathrm{H}_{0,0}+8 / 15 \zeta_{2}\left(6 x^{-2}+100 x^{-1}\right.$

$\left.+950+1360 x+885 x^{2}+216 x^{3}\right) \mathrm{H}_{-1}-1 / 45\left(-4732 x^{-1}+3042-41977 x\right.$

$\left.+46292 x^{2}\right)+8 / 45\left(-28 x^{-2}-12 x^{-1}+4281+4556 x-522 x^{2}-1008 x^{3}\right) \mathrm{H}_{-1,0}$

$\left.-96 \zeta_{2}\left(4 x-x^{2}\right) \mathrm{H}_{2}-800 \zeta_{3} x \mathrm{H}_{0}+128 \mathrm{H}_{2,1}\right)$

$+C_{A} n_{f}^{2}\left(8 / 3 p_{\mathrm{qg}}(x)\left[-3 \zeta_{2} \mathrm{H}_{1}+6 \mathrm{H}_{1,1}+4 \mathrm{H}_{1,2}+2 \mathrm{H}_{2,1}+2 \mathrm{H}_{3}\right]+8 / 3 p_{\mathrm{qg}}(-x)\left[\zeta_{2} \mathrm{H}_{-1}\right.\right.$

$\left.+2 \mathrm{H}_{-1,-1,0}+3 \mathrm{H}_{-1,0,0}\right]+8 / 3(1+2 x)\left[-2 \mathrm{H}_{-2,0}+\mathrm{H}_{-1} \zeta_{2}+2 \mathrm{H}_{-1,-1,0}-\mathrm{H}_{-1,0,0}\right]$

$+8 / 3 \zeta_{3}\left(1-46 x+10 x^{2}\right)+8 / 3 \zeta_{2}(1-2 x) \mathrm{H}_{1}-16 / 3(1+4 x) \mathrm{H}_{2,0}$

$-16 / 3 \zeta_{2}\left(1+2 x^{2}\right) \mathrm{H}_{0}+8\left(2-3 x+x^{2}\right) \mathrm{H}_{1}+8 / 9\left(-4 x^{-1}+5+38 x+39 x^{2}\right) \mathrm{H}_{0,0}$

$+16 / 9\left(-2 x^{-1}+6-15 x+11 x^{2}\right) \mathrm{H}_{2}-16 / 9 \zeta_{2}\left(2 x^{-1}+6+3 x+11 x^{2}\right)$

$+8 / 9\left(-4 x^{-1}+8-46 x+53 x^{2}\right) \mathrm{H}_{1,0}-16 / 9\left(4 x^{-1}+15+18 x+4 x^{2}\right) \mathrm{H}_{-1,0}$

$-4 / 27\left(114 x^{-1}+69+90 x-278 x^{2}\right) \mathrm{H}_{0}+2 / 81\left(-1328 x^{-1}+3105-3114 x\right.$

$\left.\left.+3524 x^{2}\right)+16 / 3\left(x^{2} \mathrm{H}_{1,0,0}-\mathrm{H}_{0,0,0}-2 \mathrm{H}_{1,1}\right)\right)$ 


$$
\begin{aligned}
+ & C_{F} n_{f}^{2}\left(8 / 3 p_{\mathrm{qg}}(x)\left[-2 \mathrm{H}_{1,0,0}-3 \mathrm{H}_{1,1}+2 \mathrm{H}_{1,1,0}-2 \mathrm{H}_{1,2}-3 \mathrm{H}_{2}+3 \mathrm{H}_{2,0}-2 \mathrm{H}_{3}\right]\right. \\
& +32 / 3 p_{\mathrm{qg}}(-x)\left[-\zeta_{2} \mathrm{H}_{-1}-2 \mathrm{H}_{-1,-1,0}+\mathrm{H}_{-1,0,0}\right]+(1-2 x)\left[-32 \mathrm{H}_{0,0,0,0}\right. \\
& \left.-16 / 3 \mathrm{H}_{1} \zeta_{2}\right]+16 / 3 \zeta_{2}\left(1-14 x+2 x^{2}\right) \mathrm{H}_{0}-4 / 9\left(8 x^{-1}+3+48 x-44 x^{2}\right) \mathrm{H}_{1,0} \\
& -16\left(3-11 x-2 x^{2}\right) \mathrm{H}_{0,0,0}+32 / 3\left(5-6 x+4 x^{2}\right) \mathrm{H}_{-2,0}+16 / 3 \zeta_{3}(7-6 x) \\
& -8 / 45 \zeta_{2}\left(40 x^{-1}+15+10 x-90 x^{2}-72 x^{3}\right)+16 / 45\left(x^{-2}-20 x^{-1}+225+40 x\right. \\
& \left.-155 x^{2}+36 x^{3}\right) \mathrm{H}_{-1,0}-8 / 45\left(40 x^{-1}+885+470 x+460 x^{2}+72 x^{3}\right) \mathrm{H}_{0,0} \\
& -4 / 135\left(712 x^{-1}+5304-6771 x-9078 x^{2}\right) \mathrm{H}_{0}-1 / 405\left(9796 x^{-1}+7731\right. \\
& \left.-151506 x+105494 x^{2}\right)-8 / 3\left(2 x^{-1}+6 x-7 x^{2}\right) \mathrm{H}_{1}+32 / 3\left(\mathrm{H}_{1,1}+\mathrm{H}_{2}\right) \\
& \left.+16 / 3 x^{2}\left[2 \zeta_{2} \mathrm{H}_{-1}+4 \mathrm{H}_{-1,-1,0}-2 \mathrm{H}_{-1,0,0}-2 \mathrm{H}_{1,0,0}-\mathrm{H}_{2,0}\right]\right) \\
+ & n_{f}^{3}\left(8 / 9 p_{\mathrm{qg}}(x)\left[-\mathrm{H}_{0,0}-\mathrm{H}_{1,0}\right]+8 / 27\left(2 x^{-1}-3+6 x\right) \mathrm{H}_{0}+4 / 81\left(13 x^{-1}-45\right.\right. \\
& \left.\left.+81 x-49 x^{2}\right)\right)
\end{aligned}
$$

and

$$
\begin{aligned}
& K_{\phi 2}^{(2)}(x)= \\
& C_{A}^{2} C_{F}\left(8 p _ { \mathrm { gq } } ( x ) \left[-8 \mathrm{H}_{1,-2,0}+2 \mathrm{H}_{1,0,0,0}+8 \zeta_{2} \mathrm{H}_{1,1}+13 / 3 \mathrm{H}_{1,1,0}-4 \mathrm{H}_{1,1,0,0}+13 / 3 \mathrm{H}_{1,2}\right.\right. \\
& \left.+8 \mathrm{H}_{1,2,0}+4 \mathrm{H}_{1,3}\right]+16 p_{\mathrm{gq}}(-x)\left[-4 \mathrm{H}_{-2,2}+8 \mathrm{H}_{-1,-2,0}-14 \zeta_{2} \mathrm{H}_{-1,-1}-8 \mathrm{H}_{-1,-1,-1,0}\right. \\
& \left.+13 \mathrm{H}_{-1,-1,0,0}+10 \mathrm{H}_{-1,-1,2}+7 \zeta_{2} \mathrm{H}_{-1,0}-5 \mathrm{H}_{-1,0,0,0}-2 \mathrm{H}_{-1,2,0}-5 \mathrm{H}_{-1,3}\right] \\
& +24(2-x)\left(-\mathrm{H}_{1,1,0}-\mathrm{H}_{1,2}\right)-104 \zeta_{3}\left(-2 x^{-1}+2-x\right) \mathrm{H}_{1}-168 \zeta_{3}\left(2 x^{-1}+2+x\right) \mathrm{H}_{-1} \\
& +8\left(32 x^{-1}+2+25 x\right) \mathrm{H}_{-2,0,0}+-4 \zeta_{2}(2+11 x) \mathrm{H}_{0,0}+4(2+13 x) \mathrm{H}_{4}-16 \zeta_{3}\left(-8 x^{-1}\right. \\
& +4-9 x) \mathrm{H}_{0}+32\left(2 x^{-1}+4+3 x\right) \mathrm{H}_{3,0}-8 \zeta_{2}\left(-8 x^{-1}+6-5 x\right) \mathrm{H}_{2}-16\left(8 x^{-1}+6\right. \\
& +5 x) \mathrm{H}_{-2,-1,0}-8(16-15 x) \mathrm{H}_{0,0,0,0}+4(18+x) \mathrm{H}_{2,0,0}-8 \zeta_{2}\left(24 x^{-1}+30+13 x\right) \mathrm{H}_{-2} \\
& -8(34-x) \mathrm{H}_{-3,0}-4 / 3 \zeta_{2}\left(-6 x^{-1}+56-7 x+16 x^{2}\right) \mathrm{H}_{1}-16 / 3\left(-20 x^{-1}+65-7 x\right. \\
& \left.+12 x^{2}\right) \mathrm{H}_{2,0}-4\left(-92 x^{-1}+75-32 x\right) \mathrm{H}_{1,0,0}+16 / 3\left(75 x^{-1}+106+50 x+8 x^{2}\right) \mathrm{H}_{-1,2} \\
& +8 / 3\left(22 x^{-1}+110+19 x-24 x^{2}\right) \mathrm{H}_{0,0,0}-8 / 3\left(58 x^{-1}+144+51 x+16 x^{2}\right) \mathrm{H}_{-1,-1,0} \\
& +4 / 3 \zeta_{2}\left(-44 x^{-1}+187+145 x+32 x^{2}\right) \mathrm{H}_{0}-4 / 3\left(44 x^{-1}+187+63 x+32 x^{2}\right) \mathrm{H}_{3} \\
& -4 / 9\left(-17 x^{-1}+242-121 x\right) \mathrm{H}_{1,1}-2 / 5 \zeta_{2}^{2}\left(384 x^{-1}+242+247 x\right)+8 / 3\left(-44 x^{-1}\right. \\
& \left.+353+41 x+48 x^{2}\right) \mathrm{H}_{-2,0}+8 / 3 \zeta_{3}\left(-347 x^{-1}+444-24 x+16 x^{2}\right)-4 / 3 \zeta_{2}\left(358 x^{-1}\right. \\
& \left.+568+251 x+48 x^{2}\right) \mathrm{H}_{-1}+8 / 9 \zeta_{2}\left(-269 x^{-1}+704+24 x+96 x^{2}\right)+4 / 3\left(632 x^{-1}\right. \\
& \left.+724+215 x+64 x^{2}\right) \mathrm{H}_{-1,0,0}-4 / 9\left(671 x^{-1}+1408+205 x+192 x^{2}\right) \mathrm{H}_{2} \\
& -4 / 9\left(1209 x^{-1}+1484+157 x+472 x^{2}\right) \mathrm{H}_{-1,0}+4 / 27\left(-5419 x^{-1}+1687+709 x\right. \\
& \left.+1446 x^{2}\right) \mathrm{H}_{1}-2 / 9\left(-1946 x^{-1}+2190-1269 x-400 x^{2}\right) \mathrm{H}_{1,0}-2 / 9\left(-1892 x^{-1}\right. \\
& \left.+10782-1667 x+1632 x^{2}\right) \mathrm{H}_{0,0}+1 / 27\left(28529 x^{-1}+27762+10188 x+15400 x^{2}\right) \mathrm{H}_{0} \\
& \left.-1 / 54\left(-223601 x^{-1}+172270-72332 x-7424 x^{2}\right)+64 \mathrm{H}_{-2,2}\right)
\end{aligned}
$$




$$
\begin{aligned}
& +C_{A} C_{F}^{2}\left(8 p _ { \mathrm { gq } } ( x ) \left[4 \mathrm{H}_{1,-2,0}-14 \zeta_{2} \mathrm{H}_{1,0}+10 \mathrm{H}_{1,0,0,0}-16 \zeta_{2} \mathrm{H}_{1,1}+11 / 3 \mathrm{H}_{1,1,0}\right.\right. \\
& \left.+16 \mathrm{H}_{1,1,0,0}+11 / 3 \mathrm{H}_{1,2}+8 \mathrm{H}_{1,3}+10 \mathrm{H}_{2,0,0}+5 \mathrm{H}_{2,1}+4 \mathrm{H}_{2,1,0}+4 \mathrm{H}_{2,2}\right] \\
& +16 p_{\mathrm{gq}}(-x)\left[-8 \mathrm{H}_{-1,-2,0}+14 \zeta_{2} \mathrm{H}_{-1,-1}+16 \mathrm{H}_{-1,-1,-1,0}-7 \mathrm{H}_{-1,-1,0,0}-6 \mathrm{H}_{-1,-1,2}\right. \\
& \left.-2 \zeta_{2} \mathrm{H}_{-1,0}-\mathrm{H}_{-1,0,0,0}-\mathrm{H}_{-1,3}\right]+16(2-x)\left(-3 \mathrm{H}_{2,1}-\mathrm{H}_{3,0}\right)-72 \zeta_{2}(2+x) \mathrm{H}_{0,0} \\
& +264 \zeta_{3}\left(-2 x^{-1}+2-x\right) \mathrm{H}_{1}+184 \zeta_{3}\left(2 x^{-1}+2+x\right) \mathrm{H}_{-1}+16(4+3 x) \mathrm{H}_{0,0,0,0} \\
& -32 \zeta_{3}\left(3 x^{-1}+5+9 x\right) \mathrm{H}_{0}+24 \zeta_{2}\left(4 x^{-1}+6+5 x\right) \mathrm{H}_{-2}-8\left(12 x^{-1}+6+11 x\right) \mathrm{H}_{-2,0,0} \\
& +16\left(12 x^{-1}+10+15 x\right) \mathrm{H}_{-2,-1,0}+8 \zeta_{2}\left(-20 x^{-1}+18-19 x\right) \mathrm{H}_{2}-32 / 3\left(11 x^{-1}+18\right. \\
& \left.+9 x+2 x^{2}\right) \mathrm{H}_{-1,2}+8(18+7 x) \mathrm{H}_{4}-8\left(6 x^{-1}+44+17 x+8 x^{2}\right) \mathrm{H}_{-2,0} \\
& +16 / 3 \zeta_{3}\left(-18 x^{-1}+56-49 x+6 x^{2}\right)+8\left(30 x^{-1}+60+13 x+8 x^{2}\right) \mathrm{H}_{-1,-1,0} \\
& -4 / 5 \zeta_{2}^{2}\left(-44 x^{-1}+72+27 x\right)-8 / 3\left(-45 x^{-1}+94-17 x+8 x^{2}\right) \mathrm{H}_{1,0,0} \\
& -2 / 3\left(-210 x^{-1}+116-23 x\right) \mathrm{H}_{1,0}-4\left(134-67 x+16 x^{2}\right) \mathrm{H}_{0,0,0}-4 / 3\left(-96 x^{-1}+142\right. \\
& -95 x) \mathrm{H}_{2,0}+4 / 15\left(16 x^{-2}-135 x^{-1}+180+205 x+36 x^{3}\right) \mathrm{H}_{-1,0}-4 / 3\left(134 x^{-1}+204\right. \\
& \left.+63 x+32 x^{2}\right) \mathrm{H}_{-1,0,0}+4 / 3 \zeta_{2}\left(-134 x^{-1}+224-61 x+24 x^{2}\right) \mathrm{H}_{1}+4 / 3 \zeta_{2}\left(178 x^{-1}\right. \\
& \left.+324+111 x+40 x^{2}\right) \mathrm{H}_{-1}-8 / 9\left(-1043 x^{-1}+420-201 x+65 x^{2}\right) \mathrm{H}_{1} \\
& +4 / 3 \zeta_{2}\left(-256 x^{-1}+556-299 x+48 x^{2}\right) \mathrm{H}_{0}-4 / 3\left(-220 x^{-1}+556-197 x+48 x^{2}\right) \mathrm{H}_{3} \\
& -4 / 9\left(-1497 x^{-1}+607-683 x-208 x^{2}\right) \mathrm{H}_{2}+4 / 45 \zeta_{2}\left(-7890 x^{-1}+3035-2800 x\right. \\
& \left.-1040 x^{2}+108 x^{3}\right)+2 / 45\left(4450+2785 x+2080 x^{2}-216 x^{3}\right) \mathrm{H}_{0,0}-2 / 45\left(-7269 x^{-1}\right. \\
& \left.+10022-6078 x+1756 x^{2}\right) \mathrm{H}_{0}-1 / 90\left(-52479 x^{-1}+133506-13606 x-3656 x^{2}\right) \\
& \left.+68 x^{-1} \mathrm{H}_{1,1}+16 x\left(-\mathrm{H}_{-3,0}+6 \mathrm{H}_{2,0,0}\right)-64 \mathrm{H}_{-2,2}\right) \\
& +C_{F}^{3}\left(8 p _ { \mathrm { gq } } ( x ) \left[14 \zeta_{3} \mathrm{H}_{1}+8 \zeta_{2} \mathrm{H}_{1,0}-4 \mathrm{H}_{1,0,0,0}+8 \zeta_{2} \mathrm{H}_{1,1}-4 \mathrm{H}_{1,1,0,0}-4 \mathrm{H}_{1,3}+3 \mathrm{H}_{2,0}\right.\right. \\
& \left.+4 \mathrm{H}_{2,1,0}+4 \mathrm{H}_{2,2}+4 \mathrm{H}_{3,0}+8 \mathrm{H}_{3,1}\right]+16 p_{\mathrm{gq}}(-x)\left[7 \zeta_{3} \mathrm{H}_{-1}+4 \mathrm{H}_{-1,-2,0}-8 \zeta_{2} \mathrm{H}_{-1,-1}\right. \\
& \left.-8 \mathrm{H}_{-1,-1,-1,0}+6 \mathrm{H}_{-1,-1,0,0}+4 \mathrm{H}_{-1,-1,2}+4 \zeta_{2} \mathrm{H}_{-1,0}-2 \mathrm{H}_{-1,0,0,0}-2 \mathrm{H}_{-1,3}\right] \\
& +64(1+x) \mathrm{H}_{-1,2}+16(2-x)\left[+2 \mathrm{H}_{2,0}+\mathrm{H}_{3}-\mathrm{H}_{3,0}-3 \mathrm{H}_{4}\right]-16(2+x) \mathrm{H}_{0,0,0,0} \\
& -16 \zeta_{2}\left(-6 x^{-1}+2-11 x\right) \mathrm{H}_{0}+16(2-9 x) \mathrm{H}_{0,0,0}+16(2-5 x) \mathrm{H}_{2,0,0}+32 \zeta_{3}\left(3 x^{-1}\right. \\
& +3+8 x)+32 \zeta_{3}\left(-2 x^{-1}+4+5 x\right) \mathrm{H}_{0}+24\left(2 x^{-1}+4+3 x\right) \mathrm{H}_{-1,0,0}+32\left(3 x^{-1}+4\right. \\
& +5 x) \mathrm{H}_{-2,0}+8(4-x) \mathrm{H}_{1,0,0}+32(4+x) \mathrm{H}_{-3,0}-16 \zeta_{2}(6-5 x) \mathrm{H}_{2}+16 \zeta_{2}(6-x) \mathrm{H}_{0,0} \\
& -16\left(6 x^{-1}+8+5 x\right) \mathrm{H}_{-1,-1,0}-8 \zeta_{2}(8-5 x) \mathrm{H}_{1}-32\left(4 x^{-1}+10+7 x\right) \mathrm{H}_{-2,-1,0} \\
& +16\left(4 x^{-1}+14+7 x\right) \mathrm{H}_{-2,0,0}-8 / 5 \zeta_{2}^{2}\left(-16 x^{-1}+16+x\right)-8 \zeta_{2}\left(6 x^{-1}+16\right. \\
& +13 x) \mathrm{H}_{-1}-4\left(-14 x^{-1}+18+x\right) \mathrm{H}_{1,0}-4\left(-10 x^{-1}+18+25 x\right) \mathrm{H}_{2}-16 \zeta_{2}\left(4 x^{-1}+18\right. \\
& +7 x) \mathrm{H}_{-2}-2\left(+27 x^{-1}+42-16 x\right) \mathrm{H}_{1}+8 / 15\left(-16 x^{-2}+285 x^{-1}+180-85 x\right. \\
& \left.-36 x^{3}\right) \mathrm{H}_{-1,0}+4 / 15 \zeta_{2}\left(420 x^{-1}+270+205 x-72 x^{3}\right)-4 / 15\left(360+85 x-72 x^{3}\right) \mathrm{H}_{0,0} \\
& -2 / 15\left(716 x^{-1}+2132+597 x-144 x^{2}\right) \mathrm{H}_{0}+2 / 15\left(-2734 x^{-1}+2716+369 x+144 x^{2}\right) \\
& \left.+16 x^{-1}\left(2 \mathrm{H}_{1,1}+3 \mathrm{H}_{1,1,0}+3 \mathrm{H}_{1,2}+6 \mathrm{H}_{2,1}\right)+128 \mathrm{H}_{-2,2}\right)
\end{aligned}
$$




$$
\begin{aligned}
+ & C_{F}^{2} n_{f}\left(16 / 3 p_{\mathrm{gq}}(x)\left[7 \zeta_{2} \mathrm{H}_{0}-2 \zeta_{2} \mathrm{H}_{1}+2 \mathrm{H}_{1,0,0}-\mathrm{H}_{1,1,0}-\mathrm{H}_{1,2}-3 \mathrm{H}_{2,0}-3 \mathrm{H}_{2,1}-7 \mathrm{H}_{3}\right]\right. \\
& +16 p_{\mathrm{gq}}(-x)\left[-\zeta_{2} \mathrm{H}_{-1}-2 \mathrm{H}_{-1,-1,0}+\mathrm{H}_{-1,0,0}\right]-32(1+x) \mathrm{H}_{-1,0}+8 / 3(2-x)\left[-2 \zeta_{2} \mathrm{H}_{0}\right. \\
& \left.+12 \mathrm{H}_{0,0,0,0}+\mathrm{H}_{2,0}+2 \mathrm{H}_{3}\right]+4 / 3\left(-18 x^{-1}+4-x\right) \mathrm{H}_{1,0}+8(8+3 x) \mathrm{H}_{0,0,0} \\
& +4 / 3\left(-91 x^{-1}+30-7 x\right) \mathrm{H}_{1}-16 / 3 \zeta_{3}\left(-26 x^{-1}+38-13 x\right)+8 / 9\left(-161 x^{-1}+110\right. \\
& -67 x) \mathrm{H}_{2}-8 / 9 \zeta_{2}\left(-161 x^{-1}+110-31 x\right)+4 / 9\left(104 x^{-1}+506+47 x\right) \mathrm{H}_{0,0} \\
& +4 / 27\left(55 x^{-1}+1401-198 x-112 x^{2}\right) \mathrm{H}_{0}+1 / 27\left(-5779 x^{-1}+13788-8223 x\right. \\
& \left.\left.+304 x^{2}\right)-24 x^{-1} \mathrm{H}_{1,1}-64 \mathrm{H}_{-2,0}\right) \\
+ & C_{A} C_{F} n_{f}\left(4 / 9 p_{\mathrm{gq}}(x)\left[-12 \zeta_{2} \mathrm{H}_{0}+42 \zeta_{2} \mathrm{H}_{1}-90 \mathrm{H}_{1,0,0}-17 \mathrm{H}_{1,1}-24 \mathrm{H}_{1,1,0}-24 \mathrm{H}_{1,2}\right]\right. \\
& +8 / 3 p_{\mathrm{gq}}(-x)\left[-\zeta_{2} \mathrm{H}_{-1}+6 \mathrm{H}_{-1,-1,0}+7 \mathrm{H}_{-1,0,0}+4 \mathrm{H}_{-1,2}+2 \mathrm{H}_{3}\right]+32 / 3\left(-3 x^{-1}+1\right. \\
& -2 x) \mathrm{H}_{2,0}+32 / 3\left(-2 x^{-1}+1-2 x\right) \mathrm{H}_{-2,0}+12(2-x) \mathrm{H}_{1,1}+8 / 9\left(-45 x^{-1}+4+17 x\right. \\
& \left.+8 x^{2}\right) \mathrm{H}_{-1,0}+16 \zeta_{3}\left(-4 x^{-1}+4-3 x\right)-32 / 3\left(x^{-1}+5+4 x\right) \mathrm{H}_{0,0,0}+8 / 9\left(-17 x^{-1}\right. \\
& \left.+160-26 x+16 x^{2}\right) \mathrm{H}_{2}-8 / 9 \zeta_{2}\left(28 x^{-1}+160-43 x+16 x^{2}\right)+8 / 9\left(-212 x^{-1}+232\right. \\
& \left.-101 x+4 x^{2}\right) \mathrm{H}_{1,0}+8 / 9\left(-229 x^{-1}+368-201 x+44 x^{2}\right) \mathrm{H}_{0,0}+4 / 27\left(26 x^{-1}+418\right. \\
& \left.-407 x-192 x^{2}\right) \mathrm{H}_{1}+4 / 27\left(-2579 x^{-1}+4572-1050 x+112 x^{2}\right) \mathrm{H}_{0} \\
& \left.+2 / 81\left(-21551 x^{-1}+11598-12585 x-6364 x^{2}\right)-32 / 3 x \mathrm{H}_{3}\right) \\
+ & C_{F} n_{f}^{2}\left(8 / 27 p_{\mathrm{gq}}(x)\left[39 \mathrm{H}_{0,0}+29 \mathrm{H}_{1}+15 \mathrm{H}_{1,0}+6 \mathrm{H}_{1,1}+12 \mathrm{H}_{2}\right]+32 / 9 \zeta_{2}\left(-2 x^{-1}+2\right.\right. \\
& \left.-x)-4 / 9\left(-119 x^{-1}+106-52 x\right) \mathrm{H}_{0}-2 / 27\left(-1003 x^{-1}+814-422 x\right)\right) .
\end{aligned}
$$

Finally the second diagonal NNLO entry is given by

$$
\begin{aligned}
K_{\phi \phi}^{(2)} & (x)= \\
C_{A}^{3} & \left(p _ { g g } ( x ) \left[18974 / 27-440 \zeta_{3}-3008 / 9 \zeta_{2}-24 / 5 \zeta_{2}^{2}-64 \mathrm{H}_{-3,0}+96 \zeta_{2} \mathrm{H}_{-2}\right.\right. \\
& +64 \mathrm{H}_{-2,-1,0}-352 / 3 \mathrm{H}_{-2,0}-64 \mathrm{H}_{-2,0,0}-64 \mathrm{H}_{-2,2}+11185 / 27 \mathrm{H}_{0}-112 \zeta_{3} \mathrm{H}_{0} \\
& -880 / 3 \zeta_{2} \mathrm{H}_{0}+3008 / 9 \mathrm{H}_{0,0}-128 \zeta_{2} \mathrm{H}_{0,0}+440 / 3 \mathrm{H}_{0,0,0}+64 \mathrm{H}_{0,0,0,0}+9782 / 27 \mathrm{H}_{1} \\
& -96 \mathrm{H}_{1} \zeta_{3}-176 / 3 \zeta_{2} \mathrm{H}_{1}-64 \mathrm{H}_{1,-2,0}+1360 / 3 \mathrm{H}_{1,0}-96 \zeta_{2} \mathrm{H}_{1,0}+616 / 3 \mathrm{H}_{1,0,0} \\
& +128 \mathrm{H}_{1,0,0,0}+968 / 9 \mathrm{H}_{1,1}+176 \mathrm{H}_{1,1,0}+128 \mathrm{H}_{1,1,0,0}+176 \mathrm{H}_{1,2}+128 \mathrm{H}_{1,2,0} \\
& +128 \mathrm{H}_{1,3}+1360 / 3 \mathrm{H}_{2}-64 \zeta_{2} \mathrm{H}_{2}+176 \mathrm{H}_{2,0}+160 \mathrm{H}_{2,0,0}+176 \mathrm{H}_{2,1}+128 \mathrm{H}_{2,1,0} \\
& \left.+128 \mathrm{H}_{2,2}+968 / 3 \mathrm{H}_{3}+160 \mathrm{H}_{3,0}+128 \mathrm{H}_{3,1}+128 \mathrm{H}_{4}\right]+8 p_{g_{2}}(-x)\left[-77 / 3 \zeta_{3}\right. \\
& -134 / 9 \zeta_{2}+11 \zeta_{2}^{2}-8 \mathrm{H}_{-3,0}+32 \zeta_{2} \mathrm{H}_{-2}+16 \mathrm{H}_{-2,-1,0}-22 \mathrm{H}_{-2,0}-36 \mathrm{H}_{-2,0,0} \\
& -24 \mathrm{H}_{-2,2}+24 \zeta_{3} \mathrm{H}_{-1}+88 / 3 \zeta_{2} \mathrm{H}_{-1}+16 \mathrm{H}_{-1,-2,0}-32 \zeta_{2} \mathrm{H}_{-1,-1}+88 / 3 \mathrm{H}_{-1,-1,0} \\
& +48 \mathrm{H}_{-1,-1,0,0}+32 \mathrm{H}_{-1,-1,2}-268 / 9 \mathrm{H}_{-1,0}+36 \zeta_{2} \mathrm{H}_{-1,0}-110 / 3 \mathrm{H}_{-1,0,0} \\
& -32 \mathrm{H}_{-1,0,0,0}-44 / 3 \mathrm{H}_{-1,2}-8 \mathrm{H}_{-1,2,0}-32 \mathrm{H}_{-1,3}-10 \zeta_{3} \mathrm{H}_{0}-11 \zeta_{2} \mathrm{H}_{0}+134 / 9 \mathrm{H}_{0,0} \\
& \left.-16 \zeta_{2} \mathrm{H}_{0,0}+11 \mathrm{H}_{0,0,0}+8 \mathrm{H}_{0,0,0,0}+4 \zeta_{2} \mathrm{H}_{2}+22 / 3 \mathrm{H}_{3}+4 \mathrm{H}_{3,0}+16 \mathrm{H}_{4}\right] \\
& +32(1+x)\left[-2 \zeta_{2} \mathrm{H}_{2}+5 \mathrm{H}_{2,0,0}+8 \mathrm{H}_{3,0}+13 \mathrm{H}_{4}\right]+64(1-x)\left[-\zeta_{2} \mathrm{H}_{-2}-2 \mathrm{H}_{-2,-1,0}\right.
\end{aligned}
$$




$$
\begin{aligned}
& \left.-3 \mathrm{H}_{-2,0,0}\right]-16\left(-11 x^{-1}+3+3 x-11 x^{2}\right) \mathrm{H}_{-1,-1,0}-8 \zeta_{2}\left(11 x^{-1}+3-3 x\right. \\
& \left.-11 x^{2}\right) \mathrm{H}_{1}-128 \zeta_{3}(3+x) \mathrm{H}_{0}+8\left(12 x^{-1}+5-5 x-12 x^{2}\right) \mathrm{H}_{1,0}-64(5-x) \mathrm{H}_{-3,0} \\
& -32 \zeta_{2}(13+11 x) \mathrm{H}_{0,0}+16 / 3\left(19+117 x-132 x^{2}\right) \mathrm{H}_{0,0,0}-16 / 3\left(-22 x^{-1}+21\right. \\
& \left.-51 x+66 x^{2}\right) \mathrm{H}_{2,0}-8\left(-33 x^{-1}+25-25 x+33 x^{2}\right) \mathrm{H}_{1,0,0}+16\left(11 x^{-1}+25+25 x\right. \\
& \left.+11 x^{2}\right) \mathrm{H}_{-1,2}-16 / 5 \zeta_{2}^{2}(33+43 x)-8 \zeta_{2}\left(11 x^{-1}+53+53 x+11 x^{2}\right) \mathrm{H}_{-1} \\
& +32 / 3\left(44 x^{-1}+57+57 x+44 x^{2}\right) \mathrm{H}_{-1,0,0}+8 \zeta_{3}\left(-55 x^{-1}+59+22 x+66 x^{2}\right) \\
& +16 / 3\left(-22 x^{-1}+62+19 x+77 x^{2}\right) \mathrm{H}_{-2,0}+8 / 3 \zeta_{2}\left(-33 x^{-1}+75-7 x+231 x^{2}\right) \mathrm{H}_{0} \\
& -8 / 3\left(11 x^{-1}+75-45 x+231 x^{2}\right) \mathrm{H}_{3}+8 / 9\left(-134 x^{-1}+137-316 x-229 x^{2}\right) \mathrm{H}_{2} \\
& -8 / 9 \zeta_{2}\left(631 x^{-1}+137+461 x-229 x^{2}\right)-8 / 3\left(255 x^{-1}+259+259 x+255 x^{2}\right) \mathrm{H}_{-1,0} \\
& -8 / 9\left(-242 x^{-1}+1593-642 x+2217 x^{2}\right) \mathrm{H}_{0,0}+2 / 27\left(-3774 x^{-1}+8194-6797 x\right. \\
& \left.+5105 x^{2}\right) \mathrm{H}_{1}+1 / 27\left(23364 x^{-1}+65830+21391 x+53565 x^{2}\right) \mathrm{H}_{0} \\
& -2 / 81\left(-129517 x^{-1}+83236-96986 x+107847 x^{2}\right)+448 x \mathrm{H}_{0,0,0,0}-1 / 486(592399 \\
& \left.\left.+38880 \zeta_{5}\right) \delta(1-x)+\left(1988 / 3 \zeta_{3}+11185 / 27 \zeta_{2}-16 \zeta_{2} \zeta_{3}-88 \zeta_{2}^{2}\right) \delta(1-x)\right) \\
& +C_{A}^{2} n_{f}\left(2 p _ { \text { gg } } ( x ) \left[-10429 / 81+24 \zeta_{3}+48 \zeta_{2}+32 / 3 \mathrm{H}_{-2,0}-2281 / 27 \mathrm{H}_{0}+80 / 3 \zeta_{2} \mathrm{H}_{0}\right.\right. \\
& -48 \mathrm{H}_{0,0}-40 / 3 \mathrm{H}_{0,0,0}-1882 / 27 \mathrm{H}_{1}+16 / 3 \zeta_{2} \mathrm{H}_{1}-512 / 9 \mathrm{H}_{1,0}-56 / 3 \mathrm{H}_{1,0,0} \\
& \left.-176 / 9 \mathrm{H}_{1,1}-16 \mathrm{H}_{1,1,0}-16 \mathrm{H}_{1,2}-512 / 9 \mathrm{H}_{2}-16 \mathrm{H}_{2,0}-16 \mathrm{H}_{2,1}-88 / 3 \mathrm{H}_{3}\right] \\
& +16 / 9 p_{\mathrm{gg}}(-x)\left[21 \zeta_{3}+10 \zeta_{2}+18 \mathrm{H}_{-2,0}-24 \zeta_{2} \mathrm{H}_{-1}-24 \mathrm{H}_{-1,-1,0}+20 \mathrm{H}_{-1,0}\right. \\
& \left.+30 \mathrm{H}_{-1,0,0}+12 \mathrm{H}_{-1,2}+9 \zeta_{2} \mathrm{H}_{0}-10 \mathrm{H}_{0,0}-9 \mathrm{H}_{0,0,0}-6 \mathrm{H}_{3}\right]+8(1+x)\left[-6 \zeta_{2} \mathrm{H}_{2}\right. \\
& \left.-4 \mathrm{H}_{2,0}+3 \mathrm{H}_{2,0,0}+3 \mathrm{H}_{4}\right]+48(1-x)\left[-\zeta_{2} \mathrm{H}_{-2}-2 \mathrm{H}_{-2,-1,0}+\mathrm{H}_{-2,0,0}\right] \\
& +32 / 3\left(x^{-1}+3+3 x+x^{2}\right) \mathrm{H}_{-1,2}+2 / 27\left(374 x^{-1}+3-807 x-1100 x^{2}\right) \mathrm{H}_{1} \\
& -8 \zeta_{2}(3+5 x) \mathrm{H}_{0,0}+16 \zeta_{3}\left(-x^{-1}+5+5 x\right)+16(5-x) \mathrm{H}_{-3,0}-16\left(-2 x^{-1}+7+7 x\right. \\
& \left.-2 x^{2}\right) \mathrm{H}_{-1,-1,0}-8 \zeta_{2}\left(2 x^{-1}+7-7 x-2 x^{2}\right) \mathrm{H}_{1}+40 / 3\left(-2 x^{-1}+17+17 x\right. \\
& \left.-2 x^{2}\right) \mathrm{H}_{-1,0}-8 / 3(20+51 x) \mathrm{H}_{0,0,0}+4 / 3\left(4 x^{-1}+21-21 x-4 x^{2}\right) \mathrm{H}_{1,0,0} \\
& +12 / 5 \zeta_{2}^{2}(23+13 x)+16 / 9\left(-35 x^{-1}+27-27 x+35 x^{2}\right) \mathrm{H}_{1,0}+8 / 3\left(-4 x^{-1}+27\right. \\
& \left.+27 x-4 x^{2}\right) \mathrm{H}_{-1,0,0}-4 / 9\left(88 x^{-1}+32+761 x-396 x^{2}\right) \mathrm{H}_{0,0}-8 / 3 \zeta_{2}\left(-2 x^{-1}+33\right. \\
& \left.+33 x-2 x^{2}\right) \mathrm{H}_{-1}+4 / 3 \zeta_{2}\left(-4 x^{-1}+39+71 x+12 x^{2}\right) \mathrm{H}_{0}-4 / 3\left(-4 x^{-1}+39+81 x\right. \\
& \left.+12 x^{2}\right) \mathrm{H}_{3}+8 / 3\left(47-5 x-12 x^{2}\right) \mathrm{H}_{-2,0}+4 / 9\left(-52 x^{-1}+145+193 x+140 x^{2}\right) \mathrm{H}_{2} \\
& -4 / 9 \zeta_{2}\left(8 x^{-1}+145-317 x+140 x^{2}\right)-2 / 27\left(2008 x^{-1}+586+3196 x-1757 x^{2}\right) \mathrm{H}_{0} \\
& -2 / 81\left(7616 x^{-1}+13155-5298 x+2473 x^{2}\right)+16 x\left[-6 \zeta_{3} \mathrm{H}_{0}+\mathrm{H}_{0,0,0,0}\right] \\
& \left.+\left(89027 / 162-80 / 3 \zeta_{3}-4562 / 27 \zeta_{2}+16 \zeta_{2}^{2}\right) \delta(1-x)\right) \\
& +C_{F}^{2} n_{f}\left(32(1+x)\left[\zeta_{2} \mathrm{H}_{0,0}-\mathrm{H}_{0,0,0,0}-\zeta_{2} \mathrm{H}_{2}+\mathrm{H}_{2,0,0}-\mathrm{H}_{3,0}-\mathrm{H}_{4}\right]\right. \\
& +32(1-x)\left[-\zeta_{2} \mathrm{H}_{-2}-2 \mathrm{H}_{-2,-1,0}+\mathrm{H}_{-2,0,0}\right]-16 \zeta_{3}\left(4 x^{-1}+1+4 x\right)
\end{aligned}
$$




$$
\begin{aligned}
& -32 \zeta_{3}(1+3 x) \mathrm{H}_{0}+\left(-4 x^{-1}+3+3 x-4 x^{2}\right)\left[-16 / 3 \zeta_{2} \mathrm{H}_{-1}-32 / 3 \mathrm{H}_{-1,-1,0}\right. \\
& \left.+16 / 3 \mathrm{H}_{-1,0,0}\right]+\left(4 x^{-1}+3-3 x-4 x^{2}\right)\left[-16 / 3 \zeta_{2} \mathrm{H}_{1}+16 / 3 \mathrm{H}_{1,0,0}\right] \\
& +16 / 5 \zeta_{2}^{2}(3-2 x)+32 / 3\left(3-2 x-4 x^{2}\right) \mathrm{H}_{-2,0}-16 / 3(3+2 x) \mathrm{H}_{0,0,0}-16 / 3\left(4 x^{-1}\right. \\
& +6+3 x) \mathrm{H}_{2,0}+16 / 3 \zeta_{2}\left(4 x^{-1}+9-10 x\right) \mathrm{H}_{0}-16 / 3\left(4 x^{-1}+9-6 x\right) \mathrm{H}_{3} \\
& +8 / 9\left(-44 x^{-1}+27-27 x+44 x^{2}\right) \mathrm{H}_{1,0}+64 / 45\left(-x^{-2}+105+95 x+9 x^{3}\right) \mathrm{H}_{-1,0} \\
& -4 / 45\left(135+1835 x-440 x^{2}+144 x^{3}\right) \mathrm{H}_{0,0}+4 / 27\left(-691 x^{-1}+675-54 x\right. \\
& \left.+70 x^{2}\right) \mathrm{H}_{1}-2 / 135\left(1884 x^{-1}+1368+6933 x+164 x^{2}\right) \mathrm{H}_{0}+-1 / 135\left(7632 x^{-1}\right. \\
& \left.+4807-3427 x-9012 x^{2}\right)-8 / 9\left(80 x^{-1}+27 x\right) \mathrm{H}_{2}+8 / 45 \zeta_{2}\left(400 x^{-1}+895 x+72 x^{3}\right) \\
& \left.+64 \mathrm{H}_{-3,0}+\delta(1-x)\right) \\
& +C_{A} C_{F} n_{f}\left(8 p_{\mathrm{gg}}(x)\left[-22 / 3+4 \zeta_{3}-\mathrm{H}_{0}-\mathrm{H}_{1}\right]+16(1+x)\left[6 \zeta_{2} \mathrm{H}_{0,0}+7 \zeta_{2} \mathrm{H}_{2}-7 \mathrm{H}_{2,0,0}\right.\right. \\
& \left.-2 \mathrm{H}_{3,0}-6 \mathrm{H}_{4}\right]+16(1-x)\left[7 \mathrm{H}_{-2} \zeta_{2}+14 \mathrm{H}_{-2,-1,0}-3 \mathrm{H}_{-2,0,0}\right]+128 \zeta_{3}(1+2 x) \mathrm{H}_{0} \\
& +64(2-3 x) \mathrm{H}_{0,0,0,0}-64 / 3\left(x^{-1}+3+3 x+x^{2}\right) \mathrm{H}_{-1,2}-4 / 3\left(32 x^{-1}+11-59 x\right. \\
& \left.+16 x^{2}\right) \mathrm{H}_{1,0}-16 / 3\left(4 x^{-1}+15-18 x-4 x^{2}\right) \mathrm{H}_{3}+16 / 3 \zeta_{2}\left(15-3 x-4 x^{2}\right) \mathrm{H}_{0} \\
& -8 / 3\left(8 x^{-1}+23-7 x\right) \mathrm{H}_{2,0}-8 / 3 \zeta_{3}\left(-20 x^{-1}+25+43 x+8 x^{2}\right)-16 / 3\left(4 x^{-1}+30\right. \\
& \left.-15 x-12 x^{2}\right) \mathrm{H}_{-2,0}-8 / 3\left(20 x^{-1}+33-33 x-20 x^{2}\right) \mathrm{H}_{1,0,0}-8 / 3(43+31 x) \mathrm{H}_{0,0,0} \\
& +16 / 3\left(-16 x^{-1}+45+45 x-16 x^{2}\right) \mathrm{H}_{-1,-1,0}-8 / 3\left(4 x^{-1}+45+45 x+4 x^{2}\right) \mathrm{H}_{-1,0,0} \\
& +8 / 3 \zeta_{2}\left(16 x^{-1}+45-45 x-16 x^{2}\right) \mathrm{H}_{1}+8 / 3 \zeta_{2}\left(-8 x^{-1}+69+69 x-8 x^{2}\right) \mathrm{H}_{-1} \\
& +16 / 9 \zeta_{2}\left(87-216 x+14 x^{2}\right)-16 / 9\left(87-3 x+14 x^{2}\right) \mathrm{H}_{2}-16 / 3(95+71 x \\
& \left.-24 x^{2}\right) \mathrm{H}_{-1,0}+4 / 9\left(-116 x^{-1}+335+1223 x+360 x^{2}\right) \mathrm{H}_{0,0}-4 / 27\left(-241 x^{-1}\right. \\
& \left.+2196-2223 x+268 x^{2}\right) \mathrm{H}_{1}-2 / 27\left(1284 x^{-1}+2463-2286 x+8464 x^{2}\right) \mathrm{H}_{0} \\
& -1 / 81\left(24445 x^{-1}+5079+31074 x-52282 x^{2}\right)-8\left(7 \zeta_{2}^{2}+12 \mathrm{H}_{-3,0}\right)+(465 / 2 \\
& \left.\left.-176 \zeta_{3}-8 \zeta_{2}\right) \delta(1-x)\right) \\
& +C_{A} n_{f}^{2}\left(4 / 81 p_{\mathrm{gg}}(x)\left[571-144 \zeta_{2}+417 \mathrm{H}_{0}+144 \mathrm{H}_{0,0}+318 \mathrm{H}_{1}+144 \mathrm{H}_{1,0}+72 \mathrm{H}_{1,1}\right.\right. \\
& \left.+144 \mathrm{H}_{2}\right]+32 / 9(1+x) \mathrm{H}_{0,0}+32 / 9\left(2-x+x^{2}\right)\left[-\zeta_{2}+\mathrm{H}_{2}\right]+2 / 27\left(52 x^{-1}+225\right. \\
& \left.-81 x+80 x^{2}\right) \mathrm{H}_{1}+2 / 27\left(192 x^{-1}+404+5 x+146 x^{2}\right) \mathrm{H}_{0}+2 / 81\left(1540 x^{-1}+891\right. \\
& \left.\left.+303 x-202 x^{2}\right)+\left(-1139 / 18-16 \zeta_{3}+556 / 27 \zeta_{2}\right) \delta(1-x)\right) \\
& +C_{F} n_{f}^{2}\left(4 p_{\mathrm{gg}}(x)+16 / 3(1+x)\left[2 \zeta_{3}+5 \mathrm{H}_{0,0,0}+\mathrm{H}_{2,0}\right]+8 / 9\left(4 x^{-1}+3-3 x\right.\right. \\
& \left.-4 x^{2}\right) \mathrm{H}_{1,0}+8 / 9\left(20 x^{-1}+47+5 x-4 x^{2}\right) \mathrm{H}_{0,0}+4 / 9\left(100 x^{-1}+155+2 x\right) \mathrm{H}_{0} \\
& \left.+2 / 27\left(1079 x^{-1}+309-1314 x+178 x^{2}\right)+\left(-43+32 \zeta_{3}\right) \delta(1-x)\right) \\
& +n_{f}^{3}\left(8 / 81 p_{\mathrm{gg}}(x)\left[-10-9 \mathrm{H}_{0}-6 \mathrm{H}_{1}\right]+8 / 81\left(2-x+x^{2}\right)\left[-10-9 \mathrm{H}_{0}-6 \mathrm{H}_{1}\right]\right. \\
& \left.+8\left(25 / 243-1 / 9 \zeta_{2}\right) \delta(1-x)\right) \text {. }
\end{aligned}
$$




\section{Appendix C: The gluon coefficient function for $F_{2}$ at large $x$}

In this final appendix we provide the large- $x$ coefficients of the gluon coefficient function $c_{2, \mathrm{~g}}^{(n)}(x)$ for the photon-exchange structure function $F_{2}$, which were not written down in Ref. [9] for brevity. As their counterparts for $c_{\phi, \mathrm{q}}^{(n)}(x)$ in Eqs. (3.22) - 3.29), these coefficients contribute to the large- $x$ behaviour of the off-diagonal physical kernels $K_{2 \phi}^{(n)}$ and $K_{\phi 2}^{(n)}$ presented above.

The second-order coefficients are (recall $L_{1} \equiv \ln (1-x)$ )

$$
\begin{aligned}
& \left.c_{2, \mathrm{~g}}^{(2)}\right|_{L_{1}^{3}}=\frac{2}{3} C_{A} n_{f}+\frac{10}{3} C_{F} n_{f}, \\
& \left.c_{2, \mathrm{~g}}^{(2)}\right|_{L_{1}^{2}}=-4 C_{A} n_{f}-9 C_{F} n_{f}, \\
& \left.c_{2, \mathrm{~g}}^{(2)}\right|_{L_{1}}=\left(14-8 \zeta_{2}\right) C_{A} n_{f}-\left(2+16 \zeta_{2}\right) C_{F} n_{f} .
\end{aligned}
$$

The corresponding three-loop results read

$$
\begin{aligned}
\left.c_{2, \mathrm{~g}}^{(3)}\right|_{L_{1}^{5}}= & \frac{2}{3} C_{A}^{2} n_{f}+\frac{10}{3} C_{F}^{2} n_{f}, \\
\left.c_{2, \mathrm{~g}}^{(3)}\right|_{L_{1}^{4}}= & -\frac{293}{54} C_{A}^{2} n_{f}-\frac{206}{27} C_{F} C_{A} n_{f}-\frac{83}{6} C_{F}^{2} n_{f}+\frac{17}{27} C_{F} n_{f}^{2}+\frac{7}{27} C_{A} n_{f}^{2}, \\
\left.c_{2, \mathrm{~g}}^{(3)}\right|_{L_{1}^{3}}= & \left.\frac{3056}{81}-\frac{136}{9} \zeta_{2}\right) C_{A}^{2} n_{f}+\left(\frac{3442}{81}-\frac{136}{9} \zeta_{2}\right) C_{F} C_{A} n_{f} \\
& +\left(\frac{127}{9}-\frac{376}{9} \zeta_{2}\right) C_{F}^{2} n_{f}-\frac{496}{81} C_{F} n_{f}^{2}-\frac{152}{81} C_{A} n_{f}^{2}, \\
\left.c_{2, \mathrm{~g}}^{(3)}\right|_{L_{1}^{2}}= & -\left(\frac{12043}{162}-\frac{232}{3} \zeta_{2}-\frac{52}{3} \zeta_{3}\right) C_{F} C_{A} n_{f} \\
& -\left(\frac{13789}{81}-\frac{652}{9} \zeta_{2}-\frac{176}{3} \zeta_{3}\right) C_{A}^{2} n_{f}+\left(\frac{1096}{81}-\frac{40}{9} \zeta_{2}\right) C_{A} n_{f}^{2} \\
& -\left(\frac{205}{6}-142 \zeta_{2}-72 \zeta_{3}\right) C_{F}^{2} n_{f}+\left(\frac{1565}{81}-\frac{16}{3} \zeta_{2}\right) C_{F} n_{f}^{2}, \\
& +\left(\frac{69526}{243}-\frac{6052}{27} \zeta_{2}-\frac{952}{9} \zeta_{3}+50 \zeta_{2}^{2}\right) C_{A}^{2} n_{f} \\
& +\left(\frac{254}{3}-\frac{46}{3} \zeta_{2}-456 \zeta_{3}+\frac{632}{5} \zeta_{2}^{2}\right) C_{F}^{2} n_{f} \\
\left.c_{2, \mathrm{~g}}^{(3)}\right|_{L_{1}}= & -\left(\frac{10798}{243}-\frac{496}{27} \zeta_{2}-\frac{16}{9} \zeta_{3}\right) C_{A} n_{f}^{2}+\left(\frac{3076}{243}+\frac{328}{9} \zeta_{2}+\frac{64}{9} \zeta_{3}\right) C_{F} n_{f}^{2} .
\end{aligned}
$$

Note the close similarity between Eqs. (3.22), (3.25), (C.1) and (C.4) for the leading logarithms at NNLO and $\mathrm{N}^{3} \mathrm{LO}$ which may point to yet another general structure. 


\section{References}

[1] D.H. Coward et al., Phys. Rev. Lett. 20 (1968) 292;

E.D. Bloom et al., Phys. Rev. Lett. 23 (1969) 930;

M. Breidenbach et al., Phys. Rev. Lett. 23 (1969) 935

[2] J.I. Friedman, H.W. Kendall, R.E. Taylor, Nobel lectures, reprinted in Rev. Mod. Phys. 63 (1991) 573, 597, 615

[3] C. Amsler et al., Particle Data Group, Phys. Lett. B667 (2008) 1

[4] D.J. Gross and F. Wilczek, Phys. Rev. Lett. 30 (1973) 1343;

H.D. Politzer, Phys. Rev. Lett. 30 (1973) 1346;

D.J. Gross and F. Wilczek, Phys. Rev. D8 (1973) 3633

[5] N. Christ, B. Hasslacher and A.H. Mueller, Phys. Rev. D6 (1972) 3543;

D.J. Gross and F. Wilczek, Phys. Rev. D9 (1974) 980;

H. Georgi and H.D. Politzer, Phys. Rev. D9 (1974) 416

[6] S. Moch, J.A.M. Vermaseren and A. Vogt, Nucl. Phys. B688 (2004) 101, hep-ph/0403192

[7] A. Vogt, S. Moch and J.A.M. Vermaseren, Nucl. Phys. B691 (2004) 129, hep-ph/0404111

[8] S. Moch, J.A.M. Vermaseren and A. Vogt, Phys. Lett. B606 (2005) 123, hep-ph/0411112

[9] J.A.M. Vermaseren, A. Vogt and S. Moch, Nucl. Phys. B724 (2005) 3, hep-ph/0504242

[10] S. Moch, J.A.M. Vermaseren and A. Vogt, Nucl. Phys. B813 (2009) 220, arXiv:0812.451768 [hep-ph]

[11] G. 't Hooft and M. Veltman, Nucl. Phys. B44 (1972) 189;

C.G. Bollini and J.J. Giambiagi, Nuovo Cim. 12B (1972) 20;

J.F. Ashmore, Lett. Nuovo Cim. 4 (1972) 289;

G.M. Cicuta and E. Montaldi, Nuovo Cim. Lett. 4 (1972) 329

[12] W.A. Bardeen, A.J. Buras, D.W. Duke, and T. Muta, Phys. Rev. D18 (1978) 3998

[13] G. 't Hooft, Nucl. Phys. B61 (1973) 455

[14] G. P. Korchemsky, Mod. Phys. Lett. A4 (1989) 1257

[15] Y.L. Dokshitzer, G. Marchesini and G.P. Salam, Phys. Lett. B634 (2006) 504, hep-ph/0511302

[16] W. Furmanski and R. Petronzio, Z. Phys. C11 (1982) 293

[17] S. Catani, Z. Phys. C75 (1997) 665, hep-ph/9609263.

J. Blümlein, V. Ravindran and W. L. van Neerven, Nucl. Phys. B586, 349 (2000), hep-ph/0004172

[18] A. Vogt, Nucl. Phys. Proc. Suppl. 79 (1999) 102, hep-ph/9906337;

J. Blümlein and A. Vogt, unpublished

[19] S. Moch and A. Vogt, JHEP 04 (2009) 081, arXiv:0902.2342 [hep-ph]

[20] S. Moch and A. Vogt, JHEP 11 (2009), 099, arXiv:0909.2124 [hep-ph]

[21] G. Sterman, Nucl. Phys. B281 (1987) 310; L. Magnea, Nucl. Phys. B349 (1991) 703;

S. Catani and L. Trentadue, Nucl. Phys. B327 (1989) 323; ibid. B353 (1991) 183;

H. Contopanagos, E. Laenen, and G. Sterman, Nucl. Phys. B484 (1997) 303, hep-ph/9604313;

S. Catani, M.L. Mangano, P. Nason and L. Trentadue, Nucl. Phys. B478 (1996) 273, hep-ph/9604351 
[22] J.R. Ellis, M.K. Gaillard and D.V. Nanopoulos, Nucl. Phys. B106 (1976) 292;

M.A. Shifman, A.I. Vainshtein, M.B. Voloshin and V.I. Zakharov, Sov. J. Nucl. Phys. 30 (1979) 711

[23] S.A. Larin, P. Nogueira, T. van Ritbergen and J. Vermaseren, Nucl. Phys. B492 (1997) 338, hep-ph/9605317;

A. Retey and J.A.M. Vermaseren, Nucl. Phys. B604 (2001) 281, hep-ph/0007294

[24] S. Moch and J.A.M. Vermaseren, Nucl. Phys. B573 (2000) 853, hep-ph/9912355

[25] S. Moch, J.A.M. Vermaseren and A. Vogt, Nucl. Phys. B726 (2005) 317, hep-ph/0506288

[26] S. Moch, J.A.M. Vermaseren and A. Vogt, Phys. Lett. B625 (2005) 245, hep-ph/0508055

[27] S. Moch and A. Vogt, Phys. Lett. B631 (2005) 48, hep-ph/0508265

[28] P.A. Baikov and K.G. Chetyrkin, Phys. Rev. Lett. 97 (2006) 061803, hep-ph/0604194

[29] S. Moch and A. Vogt, Phys. Lett. B659 (2008) 290, arXiv:0709.3899 [hep-ph]

[30] A. Daleo, A. Gehrmann-De Ridder, T. Gehrmann and G. Luisoni, arXiv:0912.0374 [hep-ph]

[31] W.E. Caswell, Phys. Rev. Lett. 33 (1974) 244;

D.R.T. Jones, Nucl. Phys. B75 (1974) 531

[32] O.V. Tarasov, A.A. Vladimirov, and A.Yu. Zharkov, Phys. Lett. B93 (1980) 429;

S.A. Larin and J.A.M. Vermaseren, Phys. Lett. B303 (1993) 334

[33] T. van Ritbergen, J.A.M. Vermaseren and S.A. Larin, Phys. Lett. B400 (1997) 379, hep-ph/9701390;

M. Czakon, Nucl. Phys. B710 (2005) 485, hep-ph/0411261

[34] K.G. Chetyrkin, B.A. Kniehl and M. Steinhauser, Nucl. Phys. B510 (1998) 61, hep-ph/9708255

[35] H. Kluberg-Stern and J.B. Zuber, Phys. Rev. D12 (1975) 467;

J.C. Collins, A. Duncan, and S.D. Joglekar, Phys. Rev. D16 (1977) 438

[36] P. Nogueira, J. Comput. Phys. 105 (1993) 279

[37] S. Moch, J.A.M. Vermaseren and A. Vogt, Nucl. Phys. B646 (2002) 181, hep-ph/0209100

[38] J.A.M. Vermaseren, math-ph/0010025

[39] J.A.M. Vermaseren, Nucl. Phys. Proc. Suppl. 116 (2003) 343, hep-ph/0211297

[40] S.G. Gorishnii et al., Comput. Phys. Commun. 55 (1989) 381

[41] S.A. Larin, F.V. Tkachev and J.A.M. Vermaseren, NIKHEF-H-91-18

[42] I. Bierenbaum, J. Blümlein and S. Klein, Nucl. Phys. B820 (2009) 417, arXiv:0904.3563 [hep-ph]

[43] P.A. Baikov et al., Phys. Rev. Lett. 102 (2009) 212002, arXiv:0902.3519 [hep-ph];

G. Heinrich, T. Huber, D.A. Kosower and V.A. Smirnov, Phys. Lett. B678 (2009) 359, arXiv:0902.3512 [hep-ph]

[44] W.L. van Neerven and A. Vogt, Nucl. Phys. B588 (2000) 345, hep-ph/0006154

[45] E. Remiddi and J.A.M. Vermaseren, Int. J. Mod. Phys. A15 (2000) 725, hep-ph/9905237

[46] T. Gehrmann and E. Remiddi, Comput. Phys. Commun. 141 (2001) 296, hep-ph/0107173

[47] T. Gehrmann, private communication

[48] S. Moch, J.A.M. Vermaseren and A. Vogt, JHEP 08 (2005) 049, hep-ph/0507039 
[49] E.A. Kuraev, L.N. Lipatov and V.S. Fadin, Sov. Phys. JETP 45 (1977) 199;

I.I. Balitsky and L.N. Lipatov, Sov. J. Nucl. Phys. 28 (1978), 822;

T. Jaroszewicz, Phys. Lett. B116 (1982) 291

[50] S. Catani, M. Ciafaloni and F. Hautmann, Nucl. Phys. B366 (1991) 135

[51] S. Catani and F. Hautmann, Nucl. Phys. B427 (1994) 475, hep-ph/9405388

[52] F. Hautmann, Phys. Lett. B535 (2002) 159, hep-ph/0203140

[53] S. Marzani et al., Nucl. Phys. B800 (2008) 127, arXiv:0801.2544[hep-ph]

[54] R.V. Harlander and K.J. Ozeren, JHEP 11 (2009) 088, arXiv:0909.3420 [hep-ph];

A. Pak, M. Rogal and M. Steinhauser, arXiv:0911.4662 [hep-ph]

[55] E.B. Zijlstra and W.L. van Neerven, Phys. Lett. B272 (1991) 127, Phys. Lett. B273 (1991) 476

[56] W.L. van Neerven and A. Vogt, Nucl. Phys. B603 (2001) 42, hep-ph/0103123

[57] P. Jimenez-Delgado and E. Reya, Phys. Rev. D79 (2009) 074023, arXiv:0810.4274 [hep-ph];

A.D. Martin, W.J. Stirling, R.S. Thorne and G. Watt, arXiv:0905.3531 [hep-ph];

S. Alekhin, J. Blumlein, S. Klein and S. Moch, arXiv:0908.2766 [hep-ph]

[58] J.A. Gracey, Phys. Lett. B322 (1994) 141, hep-ph/9401214

[59] L. Mankiewicz, M. Maul and E. Stein, Phys. Lett. B404 (1997) 345, hep-ph/9703356

[60] E. Laenen, G. Stavenga and C.D. White, JHEP 0903 (2009) 054, arXiv:0811.2067 [hep-ph]

[61] C.W. Bauer, D. Pirjol and I.W. Stewart, Phys. Rev. D65 (2002) 054022, hep-ph/0109045,

M. Beneke, A.P. Chapovsky, M. Diehl and T. Feldmann, Nucl. Phys. B643 (2002) 431, hep-ph/0206152;

A.V. Manohar, Phys. Rev. D68 (2003) 114019, hep-ph/0309176,

A. Idilbi, X.D. Ji and F. Yuan, Nucl. Phys. B753 (2006) 42, hep-ph/0605068;

T. Becher, M. Neubert and B.D. Pecjak, JHEP 01 (2007) 076, arXiv:hep-ph/0607228

[62] E. Laenen, L. Magnea and G. Stavenga, Phys. Lett. B669 (2008) 173, arXiv:0807.4412 [hep-ph];

G. Grunberg and V. Ravindran, JHEP 10 (2009) 055, arXiv:0902.2702 [hep-ph];

G. Grunberg, arXiv:0910.2894 [hep-ph] and arXiv:0911.4471 [hep-ph]

[63] P.A. Baikov and K.G. Chetyrkin, Nucl. Phys. B (Proc. Suppl.) 160 (2006) 76

[64] G. Altarelli, R.K. Ellis and G. Martinelli, Nucl. Phys. B143 (1978) 521, E.: ibid. B146 (1978) 544;

M. Diemoz, F. Ferroni, E. Longo and G. Martinelli, Z. Phys. C39 (1988) 21

[65] W.L. van Neerven and A. Vogt, Phys. Lett. B490 (2000) 111, hep-ph/0007362

[66] A. Mitov, S. Moch and A. Vogt, Phys. Lett. B638 (2006) 61, hep-ph/0604053 